

\section{Nanobubbles and nanodrops}

Joost Weijs 


\section{Samenstelling promotiecommissie:}

Prof. dr. Gerard van der Steenhoven (voorzitter) Universiteit Twente

Prof. dr. rer. nat. Detlef Lohse (promotor)

Universiteit Twente

Dr. ir. Jacco H. Snoeijer (assistent promotor)

Universiteit Twente

Prof. dr. ir. Harold J.W. Zandvliet

Universiteit Twente

Prof. dr. Claudia Filippi

Prof. dr. L. Gary Leal

Prof. dr. ir. Thijs J. H. Vlugt

Universiteit Twente

UC, Santa Barbara

Technische Universiteit Delft
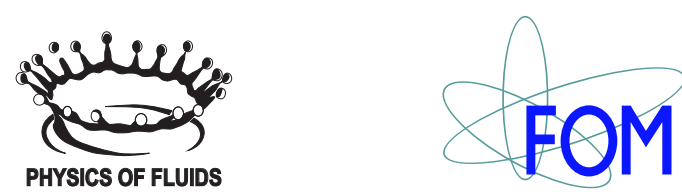

The work in this thesis was carried out at the Physics of Fluids group of the Faculty of Science and Technology of the University of Twente. It is part of the research programme of the Foundation for Fundamental Research on Matter (FOM), which is financially supported by the Netherlands Organisation for Scientific Research (NWO).

Nederlandse titel: Nanobellen en nanodruppels

Publisher: Joost H. Weijs, Physics of Fluids, University of Twente, P.O. Box 217, 7500 AE Enschede, The Netherlands pof.tnw.utwente.nl

Cover: Impression of nanoscopic capillarity phenomena. Front: Liquid nanodrop on a deformable substrate. Back: Surface nanobubble on a deformable substrate.

Print: Gildeprint Drukkerijen, Enschede

(C) Joost H. Weijs, Enschede, The Netherlands, 2013

No part of this work may be reproduced by print photocopy or any other means without the permission in writing from the publisher

ISBN: 978-90-365-0667-0

DOI: $10.3990 / 1.9789036506670$ 


\section{NANOBUBBLES AND NANODROPS}

\section{PROEFSCHRIFT}

ter verkrijging van

de graad van doctor aan de Universiteit Twente,

op gezag van de rector magnificus,

Prof. dr. H. Brinksma,

volgens besluit van het College voor Promoties

in het openbaar te verdedigen

op woensdag 25 september 2013 om 16.45 uur

door

Joost Hidde Weijs

geboren op 16 september 1984

te Tiel 
Dit proefschrift is goedgekeurd door de promotor:

Prof. dr. rer. nat. Detlef Lohse

en de assistent-promotor:

Dr. ir. Jacco Snoeijer 
Contents

1 Introduction 1

1.1 Interfaces at the nanoscale . . . . . . . . . . . . . . I

1.2 Surface nanobubbles ................ . . . . 4

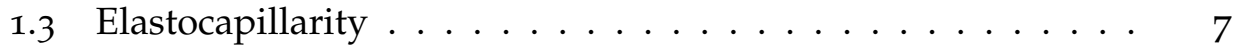

1.4 Guide through this thesis ............. 10

2 Formation, contact angle and lifetime of surface nanobubbles $\quad 17$

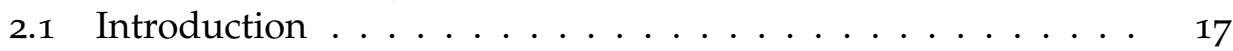

2.2 Numerical setup . . . . . . . . . . . . . . . . 19

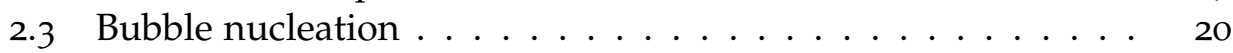

2.4 Universal contact angle . . . . . . . . . . . . 22

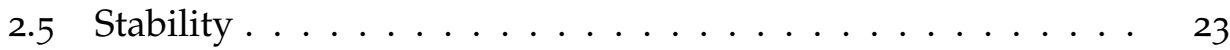

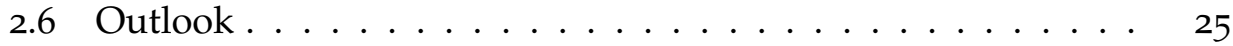

3 Diffusive shielding stabilizes bulk nanobubble clusters 29

3.1 Introduction ................... 29

3.2 Numerical details . . . . . . . . . . . . . . . . . 32

3.3 Results from the MD simulations ........... 33

3.4 Continuum description . . . . . . . . . . . . 35

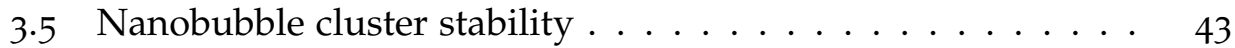

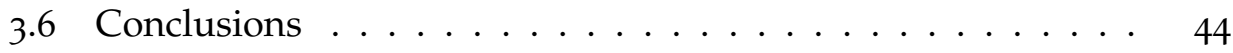

4 Why surface nanobubbles live for hours 49

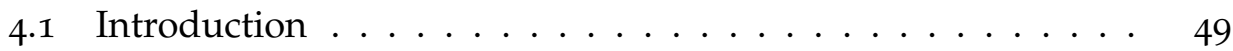

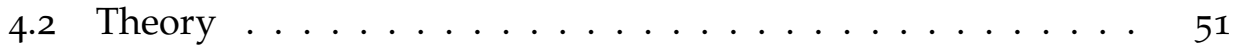

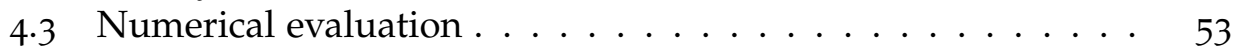

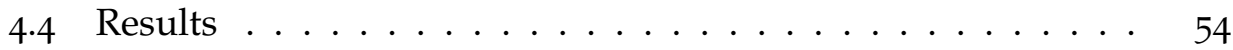

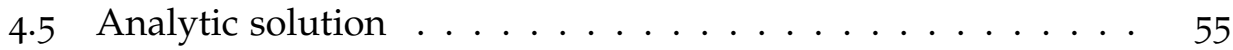

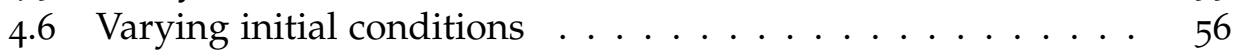

4.7 Robustness of the results ............. 56 


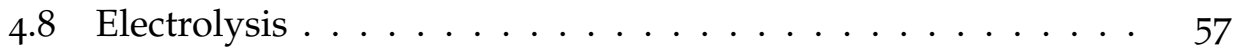

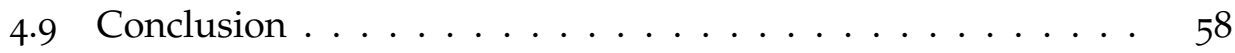

5 Rectified Diffusion $\quad 61$

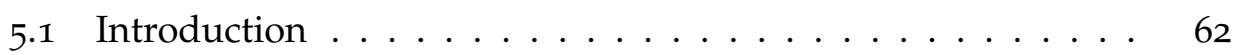

5.2 Experimental observations . . . . . . . . . . . . 63

5.3 Mathematical formulation of the problem . . . . . . . 66

5.4 Numerical evaluation . . . . . . . . . . . . . . . . . 68

5.5 Analytical derivation of the bubble growth rate $\ldots \ldots 70$

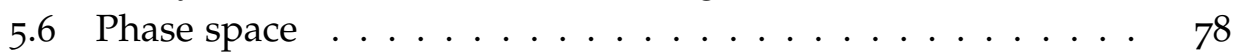

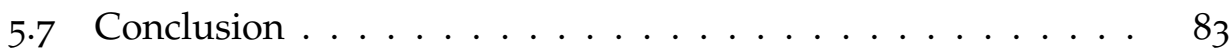

6 Why is surface tension a force parallel to the interface? 87

6.1 Basic concepts and problems . . . . . . . . . . 87

6.2 Microscopic interpretation of capillarity . . . . . . . . . . 92

6.3 Microscopic interpretation of wetting ........ 100

6.4 Conclusion . . . . . . . . . . . . . . 111

7 Origin of line tension for a Lennard-Jones nanodroplet $\quad 115$

7.1 Introduction . . . . . . . . . . . . . . 115

7.2 Nanodrops from Molecular Dynamics . . . . . . . . . 118

$7 \cdot 3$ Origin of line tension effect $\ldots \ldots \ldots \ldots \ldots \ldots$

$7 \cdot 4$ Discussion . . . . . . . . . . . . . 137

8 Elastocapillarity at the nanoscale: on the coupling between elastic$\begin{array}{ll}\text { ity and surface energy in soft solids } & 145\end{array}$

8.1 Introduction . . . . . . . . . . . . . 146

8.2 Drops and bubbles . . . . . . . . . . . . . . . . . 149

8.3 Partially immersed solid . . . . . . . . . . . . . . . . . . . . . 155

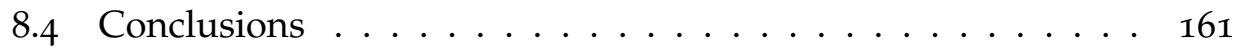

9 Capillarity of soft amorphous solids: a microscopic model for sur$\begin{array}{ll}\text { face stress } & 171\end{array}$

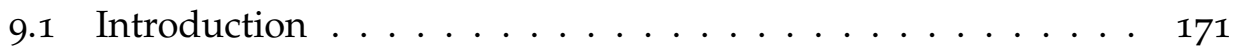

9.2 Microscopic model . . . . . . . . . . . . . . . . . 173

9.3 Excess quantities and surface stress $\ldots \ldots \ldots \ldots$. . . . . . . . . . .

9.4 Discussion ...................... 186 
10 Initial spreading of low-viscosity drops on partially wetting surfaces

10.1 Introduction . . . . . . . . . . . . . . . . . . 193

10.2 Molecular Dynamics simulations . . . . . . . . . . . 195

10.3 Experiments . . . . . . . . . . . . . . . . . . . . . 199

10.4 Discussion . . . . . . . . . . . . . . 202

11 Summary and Outlook 205

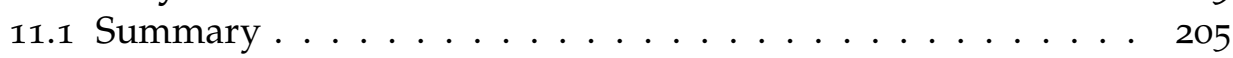

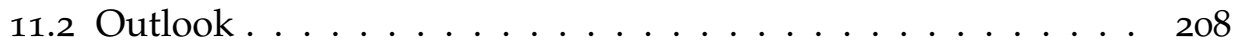

$\begin{array}{ll}\text { Samenvatting } & 211\end{array}$

$\begin{array}{ll}\text { Acknowledgements } & 215\end{array}$

$\begin{array}{ll}\text { About the author } & 217\end{array}$ 


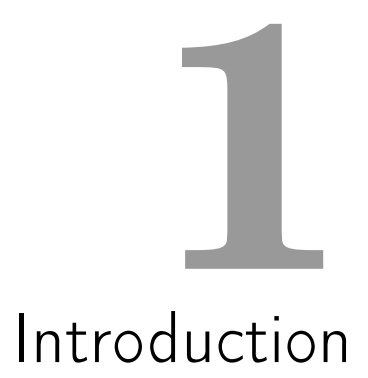

\subsection{Interfaces at the nanoscale}

For any material around us, the interaction between individual atoms is responsible for the behaviour of that material. Even though the length-scales involved in the atomic interaction are only several nanometers, the manifestations can be observed at macroscopic scales. In the context of this thesis, we focus on capillarity. The microscopic origin of capillarity is due to absent atomic interactions near an interface compared to the interactions in the bulk, as sketched in Fig. 1.1(a). However, the effects of capillarity have been described long before the existence of atoms was even known [1, 2], which shows that using macroscopic continuum concepts we can describe the world around us very well $[3,4]$. There are limits, of course: the continuum description cannot hold at arbitrary scales as at some point the discrete, atomistic nature of the materials becomes noticeable. Experimentally, we are able to access increasingly small length-scales in solid and fluid matter $[5,6]$, and a natural question one can ask is: When does the continuum model fail and when should we account for the atomic nature of the involved material?

A recent example is the discovery of surface nanobubbles [7-9]. Surface nanobubbles are bubbles of height $\sim 10 \mathrm{~nm}$ and are found on various solid-water interfaces. Surface nanobubbles possess some unexpected 
characteristics and non-continuum, microscopic descriptions are explored in order to explain some of these properties. Some theories, for example, invoke line tension which is a capillary effect that starts to play a role at the nanoscale [10-13] and is notoriously difficult to measure [14-17]. Only recently, experimental methods have allowed for a direct measurement of line tension and confirm that it is indeed an effect that only starts to play a role at the nanoscale [18]. Another current 'hot' research topic is the field of elastocapillarity, where the effects on a partially wetted, elastic solid due to the presence of the liquid are explored. Although original research on the elastic response to capillary forces dates back to the 1960's [19, 20], recent developments in experimental techniques have re-established interest [2124]. It turns out that a full understanding of the elastic response to capillary forces is elusive, with varying interpretations. Understanding the involved microscopics is the way to obtain a consistent macroscopic description of elastocapillarity. Another field, electrowetting, involves altering the wetting properties of a liquid by applying an electric field. Although macroscopic theory can be used to describe the general behaviour of electrowetting it fails on some crucial points [25]. Therefore, also here, a study at the microscopic level reveals answers that the macroscopic description could not [26]. As a final example we briefly discuss slip at solid-fluid interfaces. Experiments reveal that for all materials there exists some finite slip, i.e. the fluid 'slides' over the surface which becomes important when the outer flow-dimensions are comparable to the slip length such as in micro- and nanofluidic devices [27]. The study of slip inherently involves nanoscopic length-scales, certainly so for atomically smooth surfaces such that also here a microscopic approach (for example using molecular dynamics simulations) has proven very effective $[28,29]$.

From the examples above we clearly see that nanoscopic insights into physical phenomena are crucial to characterize the full behaviour of a system. Molecular dynamics turns out to be an extremely valuable tool to gain those microscopic insights as it directly accesses the relevant length scales. To illustrate this, we return to the example of surface tension at a liquidvapour interface, which originates at the nanoscale and is therefore naturally accessible by molecular dynamics. In Fig. 1.1(b) a snapshot of a molecular dynamics simulation of a straight liquid-vapour interface is shown for a "simple" (mono-atomic) liquid. The two phases can clearly be distinguished due to the large difference in number density of atoms, Fig. 1.I(c). Even so, the thermodynamic pressures in the bulk of both phases are equal as the 

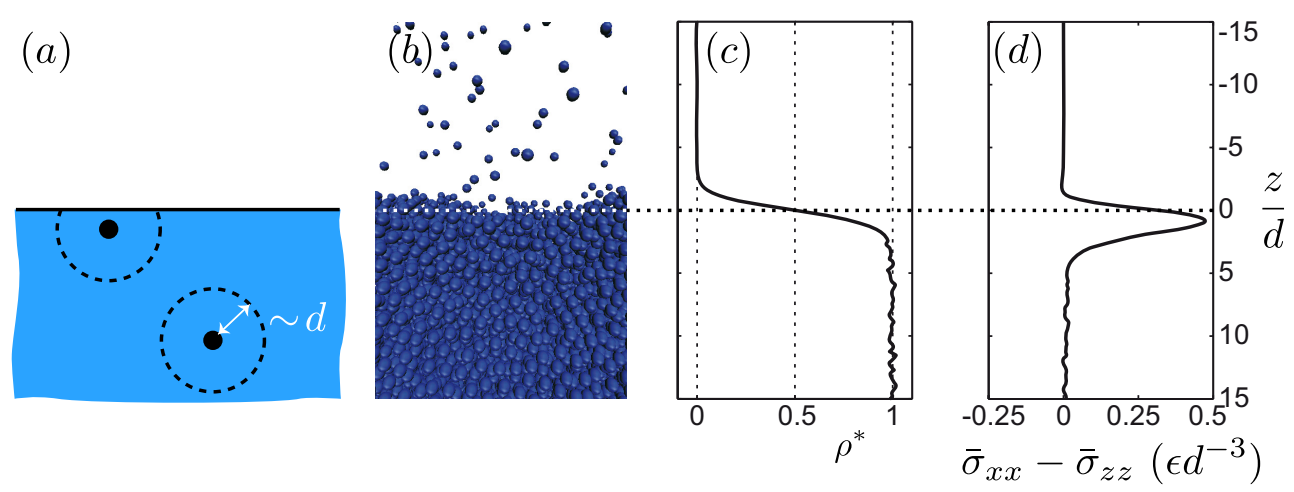

Figure 1.1: Liquid-vapour interface. (a) Schematic depiction of the microscopic origin of surface tension. Atoms near the interface miss a portion of their interactions compared to bulk atoms which leads to a surface energy. (b) Snapshot from molecular dynamics of a Lennard-Jones liquid in contact with its own vapour. (c) Time-averaged density profile $\rho^{*}=\left(\rho(z)-\rho_{V}\right) /\left(\rho_{L}-\rho_{V}\right)$ of the molecular dynamics simulation from (b), with $\rho(z)$ the local density, $\rho_{V}$ the bulk vapour density, and $\rho_{L}$ the bulk liquid density. $\rho^{*}=0$ corresponds to the vapour density and $\rho^{*}=1$ to the liquid density. Note that there exists a smooth transition over a a few molecular diameters between the two bulk densities. (d) Stress anisotropy around the interface from (b). In the bulk phases, the stress is isotropic, but near the interface there is a strong tensile stress parallel to the interface which corresponds to the surface tension. Stresses are given in units of $\epsilon / d^{3}$, with $\epsilon$ the molecular interaction energy and $d$ the molecular size.

system is in equilibrium. To evaluate this pressure we can take a thermodynamic approach by calculating the time-averaged stress-tensor $\bar{\sigma}(z)$, Fig. 1.1(d). We then find that, although isotropic and constant in the bulk, the elements of $\bar{\sigma}$ tangential to the interfaces $(x, y)$ deviate around the interface [30]. This is a direct effect of the broken symmetry induced by the presence of the interface [Fig. 1.1(d)], and the integral over this 'excess' stress (compared to the bulk value) is the surface tension:

$$
\mathrm{Y}=\int\left[\bar{\sigma}_{x x}(z)-\bar{\sigma}_{z z}(z)\right] \mathrm{d} z
$$

Note that for this geometry $\sigma_{x x}(z)=\sigma_{y y}(z)$ and can therefore be interchanged. Equation (1.1) is known as the Kirkwood-Buff integral, and shows that in the case of a liquid-fluid interface the surface free energy per unit area represents an actual stress parallel to the interface, and that both are equal [31]. 
Molecular dynamics, therefore, confirm that the origin of surface tension is microscopic and it recovers the well-known macroscopic manifestations of surface tension, such as stress, surface energy and pressure.

Since many chapters in this thesis contain molecular dynamics results we briefly discuss this simulation technique. The basic idea of molecular dynamics is to numerically solve Newton's equations of motion for an $\mathrm{N}$ body system where the particles (atoms or molecules) interact with each other with some defined potential. Throughout this thesis, for two particles at distance $r$ from each other, we use the Lennard-Jones potential

$$
\phi_{L J}(r)=4 \epsilon\left[\left(\frac{d}{r}\right)^{12}-\left(\frac{d}{r}\right)^{6}\right]
$$

to model the short range repulsion due to finite size $\left(\sim r^{-12}\right)$ and the longranged van der Waals attraction $\left(\sim-r^{-6}\right)$ of atoms. $\epsilon$ (unit energy) then sets the strength of the interaction and $d$ (unit length) the atom size. Although such "simple" fluids do not represent a real-world material such as water, we can use it since we are not interested in studying specific material properties but rather to gain some completely generic microscopic insights in fluids. If, a priori, it is unknown whether continuum models are valid at the scales that are investigated molecular dynamics can be used as it models the materials at the atomic scale. A good example is a moving contact line: hydrodynamically the shear stress diverges near the contact line (as the liquid layer height goes to zero). Therefore, in order for the contact line to be able to move one needs to invoke slip or some other way to regularize the diverging stress. This is of course not required in molecular dynamics, where this divergence does not exist in the same way as it does not exist for a physical system: there is a limiting small scale set by the atomic size. In this sense, molecular dynamics can be thought of as an experiment: the collective (microscopic) behaviour of the atoms manifests in macroscopic behaviour which can be tested against predictions. In the following sections we discuss surface nanobubbles and elastocapillarity. In both cases we have used molecular dynamics to study the microscopic characteristics and how they are related to the available macroscopic descriptions.

\subsection{Surface nanobubbles}

Surface nanobubbles are flat bubbles (gas side contact angle $\sim 20^{\circ}$ ) and can be observed by atomic force microscopy on water-solid interfaces $[7,8,32]$. 
Typically, the lateral size of nanobubbles ranges from $10 \mathrm{~nm}-1 \mu \mathrm{m}$ and their height is several tens of nanometers. The lifetime of macroscopic bulk bubbles is well understood but, as explained below, when applying this estimate to surface nanobubbles one finds that nanobubbles should exist for no more than a millisecond, whereas they are experimentally found to live for hours to even days $[33,34]$. This discrepancy of over eight orders of magnitude is undoubtedly the biggest mystery about surface nanobubbles. Apart from the fundamental questions, there are many practical reasons why surface nanobubbles are of interest. Say, for example, that one would like to create nanobubbles to facilitate drag reduction in microfluidic devices [35]. To do so in a reproducible and efficient way it is necessary to obtain a deep understanding of nanobubbles. Conversely, some industrial processes, for example lithographic systems in the semiconductor industry, are hindered by the presence of surface nanobubbles. In this case a deep insight in nanobubbles may provide a clean, fast and efficient way to dispose them [36].

Although many aspects of surface nanobubbles are unpredicted and unexplained (such as their low gas-side contact angles [37], their 'superstability' [38], their micropancake 'cousins' [39], and their apparent resilience to Ostwald-ripening) the most surprising feature is their lifetime of hours up to days. All free bubbles dissolve (as long as the surrounding liquid is not supersaturated) because there exists a positive feedback due to the Laplace pressure:

$$
\Delta p_{L}=\frac{2 \gamma}{R},
$$

with $R$ the bubble radius. For a nanobubble therefore, with $R=100 \mathrm{~nm}$ this would imply an excess pressure of about one to two atmospheres, which increases even more as the bubble shrinks. This, in turn, causes a higher concentration of dissolved gas in the vicinity of the bubble wall owing to Henry's law, and the diffusive flux of gas away from the bubble will increase in strength increasing the rate of bubble reduction. To obtain an estimate for the bubble lifetime, we should therefore look at the diffusive transport of gas away from the bubble. The diffusion equation reads, in spherical coordinates:

$$
\frac{\partial c}{\partial t}=\frac{D}{r^{2}} \frac{\partial}{\partial r}\left(r^{2} \frac{\partial}{\partial r} c\right)
$$

where $c$ is the dissolved gas concentration and $D$ the diffusion constant. From dimensional analysis we then get the following dependence of the 

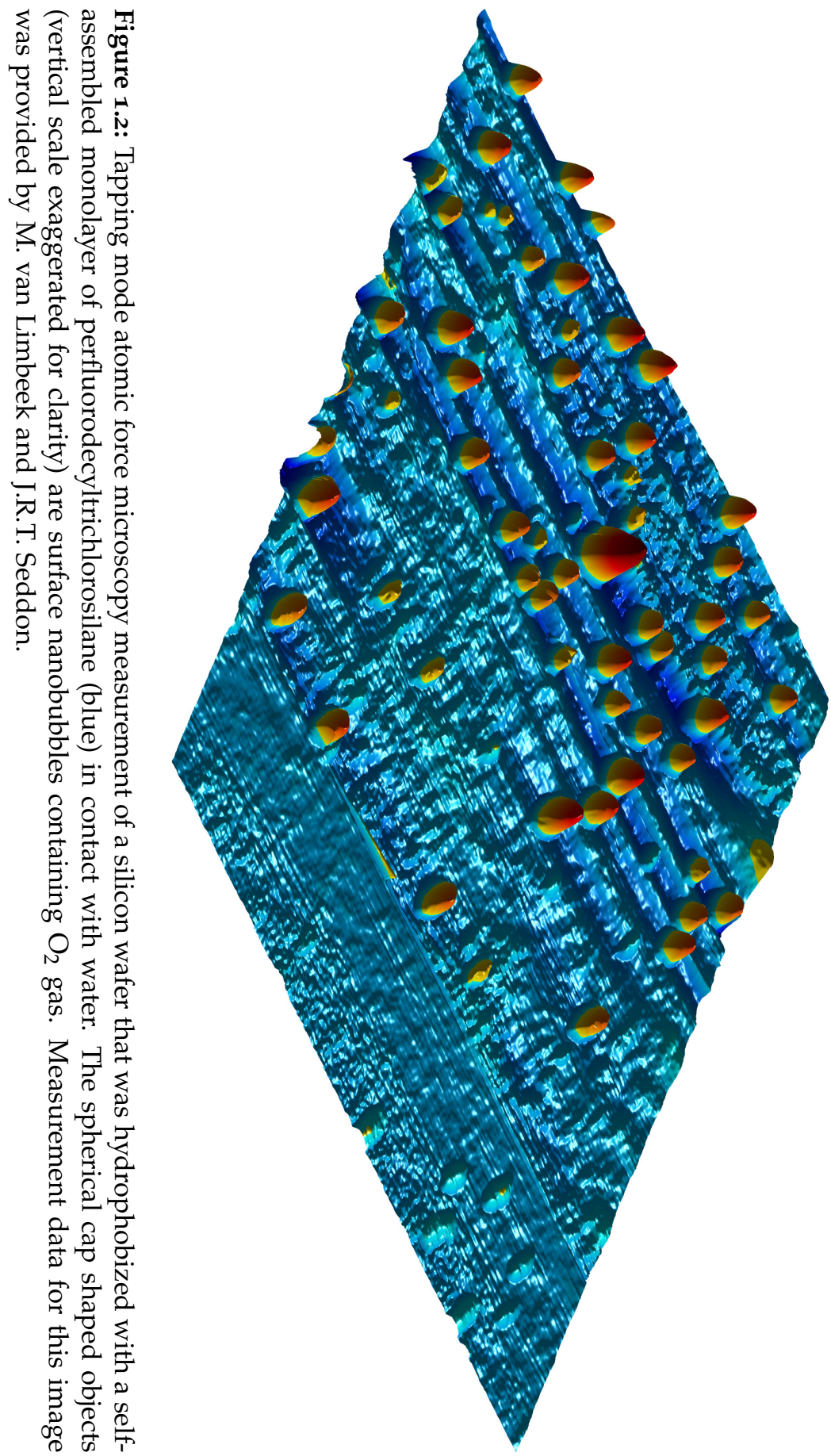
lifetime on the initial size $R_{0}$ and $D$ :

$$
\tau \sim \frac{R_{0}^{2}}{D}
$$

In the case of nanobubbles, the spherical symmetry is of course lost but the scaling behaviour should still hold and we find lifetimes of order $10^{-5}$ $10^{-3} \mathrm{~s}$.

This large discrepancy with the experimental findings have sparked numerous hypotheses to explain the long lifetimes. They can broadly be divided into two categories: static equilibrium and dynamic equilibrium. In the case of static equilibrium the outflux of gas from the bubble is partly or completely suppressed [40]. The responsible mechanism is unclear; a reduction in the surface tension (due to contaminants) alone cannot account for several orders of magnitude of lifetime increase. A complete coverage of the bubble-liquid interface by contaminate surfactants would block the gas flow through the interface completely but it is unknown why such an effect would only occur for nanobubbles. Furthermore, systematic studies where the contaminant level was controlled did not reveal any effect of contaminant concentration on nanobubbles [41].

Another theory for the stability of nanobubbles claims that the gas outflux is compensated by a gas inflow back into the bubbles [10, 42]. Hence, when in- and outflux of gas balance a dynamic equilibrium is reached and the bubble does not grow or shrink any more. To date, there is no direct experimental evidence that such a recirculation exists and, more importantly, it is unknown what would drive this flow.

\subsection{Elastocapillarity}

In the case of a liquid partially wetting a solid we are used to think about how the liquid is affected by the solid, and we describe only the final shape of the droplet: usually a spherical cap with Young's contact angle $\theta_{Y}$. However, it is clear that the liquid also affects the solid: Capillary forces attempt to deform the solid while the solid resists due to elasticity, hence the name elastocapillarity [19-24, 43,44]. The typical length-scale of deformation is therefore naturally

$$
\Delta h_{e c} \sim \frac{\gamma}{E},
$$

with $E$ the elastic modulus and $\gamma$ the surface free energy of the liquid. In the case of a stiff solid this effect is not very pronounced: for water on 

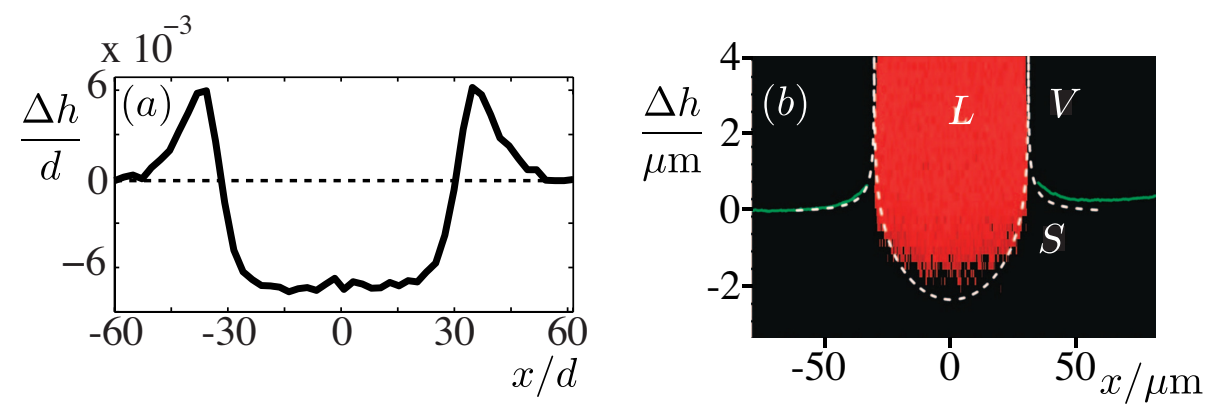

Figure 1.3: Elastocapillary deformation of an elastic solid due to a sessile drop. (a) Molecular dynamics result of an elastic solid in contact with a Lennard-Jones liquid. In SI-units, at temperature $T=300 \mathrm{~K}$ and taking $d=0.34 \mathrm{~nm}$ the elastic modulus of the solid would be $E=11 \mathrm{GPa}$ and the surface tension of the liquid $\gamma=3.1 \cdot 10^{-2} \mathrm{~J} / \mathrm{m}^{2}$. The contact lines are located at $x \approx \pm 35 d$, clearly showing how the solid gets pulled upwards due to the contact line. In the center of the drop $|x|<35 d$ the solid is pushed downwards due to the Laplace pressure of the liquid in the droplet. Note the small length-scales: $d$ is the diameter of a liquid atom [defined in Eq. (1.2)]. Time-averaging was used to filter the noise due to thermal fluctuations. (b) Experimental result of a sessile droplet $\left(\gamma=4.9 \cdot 10^{-2} \mathrm{~J} / \mathrm{m}^{2}\right)$ in contact with a silicone elastomer $(E=25 \mathrm{kPa})$. Although the length-scales are completely different compared to the molecular dynamics case, a similar shape is observed. Image adapted from [21].

steel the solid gets deformed by fractions of an Angstrom. In molecular dynamics such small deformations can be measured after sufficient temporal averaging [Fig. 1.3(a)]. For soft solids such as elastomers or gels, for which the elastic modulus can be as low as $10 \mathrm{kPa}$, the deformation will be up to tens of microns. This can indeed be measured experimentally [21, 22, 24], as shown in Fig. 1.3(b). Here, a very intuitive shape of the solid is recovered: a depression in the center due to the Laplace pressure of the liquid, and a ridge at the contact line where surface tension pulls on the solid.

So how does one characterize this elastocapillary behaviour? In this thesis we show using theoretical approaches (both microscopic and thermodynamic) and molecular dynamics that the elastic properties of a solid play a crucial role in elastocapillarity, which means that, once more, the microscopic physics of the interface needs to be considered. A key property that has been completely overlooked in the field of elastocapillarity is that, in general, one cannot equate the surface free energy $(\gamma)$ to the surface stress 
$(\mathrm{Y})$ at the interface. This result contrasts what we found in Sect. 1.1, where we saw that in the case of two fluids (so no elasticity) surface stress and surface energy are equal. In fact, it is important to note that the Kirkwood-Buff integral (1.1) does not compute the surface energy, but rather the surface stress. The distinction between surface energy and surface stress must be made in the case where one of the phases is an elastic solid. The surface stress $Y$ can, however, be related to the surface energy $\gamma$ in the following way [45]:

$$
\mathrm{Y}=\gamma+\frac{\mathrm{d} \gamma}{\mathrm{d} \varepsilon}
$$

with $\varepsilon$ the (elastic) strain parallel to the interface. This relation is known as the Shuttleworth equation, and it recovers that for fluid-fluid interfaces $\mathrm{Y}=\gamma$, since there is no strain dependence of the surface energy due to the lack of elasticity.

Before we discuss the consequences of Eq. (1.7) we derive it below which helps to understand the physical meaning of this relation. To do this, we consider the solid block shown in Fig. 1.4. Cutting this block in half adds $2 \gamma A_{0}$ to its interfacial energy as shown in the figure. Then, to compress the two halfs requires work $W_{0}$ to overcome the bulk elasticity. In addition, the surface stress of the newly created interface has to be overcome requiring an additional amount of work $2 \mathrm{Yd} A$. The total additional energy in the material due to these actions is therefore:

$$
W_{I}=2 \gamma A_{0}+2 \gamma \mathrm{d} A+W_{0} .
$$

The next step is to repeat the above procedure, but this time we apply the compression first which requires work $W_{0}$ as there is not yet (newly created) interfacial area along which a surface stress acts. Due to this, the side areas of the block have changed by $\mathrm{d} A$, and the surface free energy will have changed to $\gamma+\mathrm{d} \gamma$. Therefore, due to this compression the surface energy that is now associated with a cut in the $y z$-plane is $2[\gamma+(\mathrm{d} \gamma / \mathrm{d} \varepsilon) \mathrm{d} \varepsilon]\left[A_{0}+\right.$ $\mathrm{d} A]$.

$$
W_{I I}=2(\gamma+\mathrm{d} \gamma)\left(A_{0}+\mathrm{d} A\right)+W_{0} .
$$

Both procedures lead to the same end state so $W_{I}=W_{I I}$. Using $(\mathrm{d} \varepsilon / \mathrm{d} A)=$ $1 / A_{0}$ this can then be rewritten as the Shuttleworth equation (1.7).

An important consequence of the general inequality $\gamma \neq \mathrm{Y}$ is that Young's law, which depends on the surface energies, is by itself not sufficient to fully describe an elastocapillary problem since the mechanical equilibrium in the solid is determined by the surface stress. For example, when we compare a 


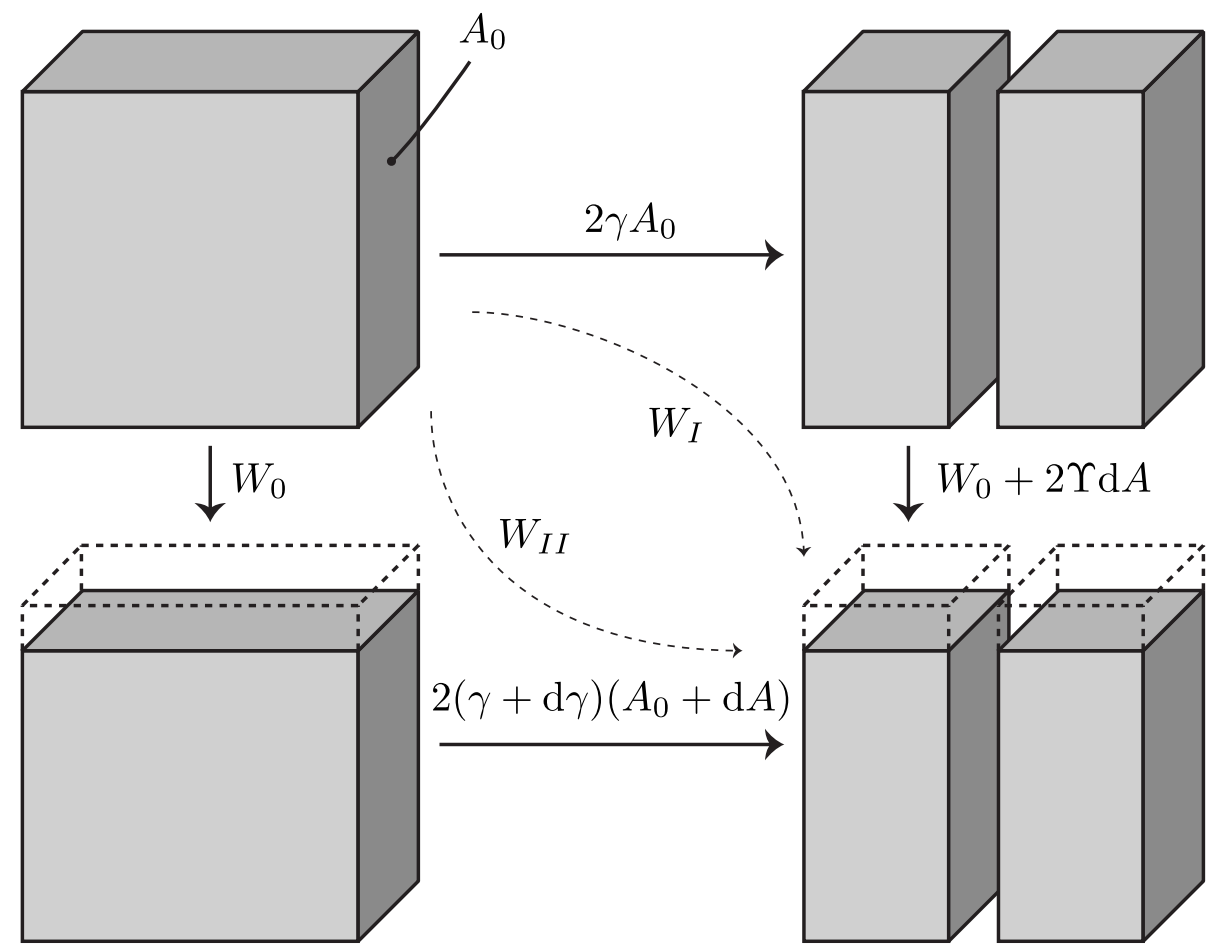

Figure 1.4: Derivation of the Shuttleworth relation. Image adapted from [46].

droplet or a bubble on a surface at $\theta_{Y}=90^{\circ}$, the elastic response in the solid is found to be completely different in both cases. This proves that surface energies (which are equal in both cases) do not fully determine elastocapillary behaviour.

\subsection{Guide through this thesis}

This thesis is divided into two parts: Chapters 2-5 deal with nanobubbles, and chapters 6-10 describe studies on the microscopic origins of capillarity phenomena in a more general fashion.

Chapter 2 contains a study, using molecular dynamics, of the formation, contact angle and stability of surface nanobubbles. By simulating supersaturated liquid mixtures in contact with a solid, we investigate under which conditions surface nanobubbles can form. The nanobubbles that are created 
in this way can then be compared against their real-life counterparts, and it turns out that a high gas-solid affinity can result in the low (gas side) contact-angles that are observed in experiments. Finally, we look into the lifetimes of the simulated nanobubbles and at the details of gas transport across the liquid-gas interface.

In chapter 3 we use molecular dynamics to study bulk nanobubbles. In the case of bulk nanobubbles there is no wall such that the bubbles are spherical, and an isolated bubble would dissolve according to the EpsteinPlesset model [33]. In this case however, we place the nanobubble in a bubble cloud, to study how the presence of neighbouring bubbles affect the lifetime.

Then, in chapter 4 we describe a novel nanobubble model, using solely macroscopic concepts such as diffusion and some geometric properties of nanobubble shrinkage to explain the long lifetimes of surface nanobubbles.

Finally, in chapter 5 we study the growth of surface nanobubbles that are subjected to an ultrasonic acoustic field [47]. We combine experimental, numerical and analytical approaches to gain a quantitative understanding of rectified diffusion for nanobubbles.

In the second part of the thesis we address microscopic aspects of capillarity in a more general context. In chapter 6 we reconcile the thermodynamic, mechanical and microscopic descriptions of capillarity and wetting, by posing some general questions that anyone who studies capillarity faces. By connecting these descriptions we develop a consistent framework to tackle capillarity problems at the microscopic scale.

Then, in chapter 7 we use this microscopic description to study the origin of line tension. Using molecular dynamics, we directly measure this line tension just as done in previous experiments, namely, by measuring the contact angle as a function of drop size, and find consistent results.

Chapters 8 and 9 deal with elastocapillarity: The interaction between a liquid and a solid that is partially wetted by this liquid. As already mentioned, the solid is affected (deformed) by the presence the liquid. In chapter 8 we provide a thermodynamic description of the stress exerted on the solid by the liquid. Then, in chapter 9 we tackle this problem from the microscopic perspective using density functional theory in the sharp kink approximation. Using this method, we find that the Poisson ratio of the solid $v$ is the key parameter that determines the surface stress behaviour. For example, in the case $v=1 / 2$ (incompressible solid) we recover $Y=\gamma$, as in the liquid-fluid case.

The final chapter contains a brief study about spreading. As a droplet 
is brought into contact with a solid a singular point with infinite interfacial curvature is created which causes rapid contact line motion. We measured this contact line motion both in experiments and in molecular dynamics simulations and find an excellent agreement with scaling laws, where the contact line radius of the droplet increases with the square root of time.

\section{References}

[1] P.-G. de Gennes, Wetting: statics and dynamics, Rev. Mod. Phys. 57, 827 (1985).

[2] T. Young, An essay on the cohesion of fluids, Phil. Trans. R. Soc. London 95, 65 (1805).

[3] P.-G. de Gennes, F. Brochard-Wyart, and D. Quere, Capillarity and wetting phenomena: drops, bubbles, pearls, waves (Springer, New York, 2004).

[4] D. Bonn, J. Eggers, J. Indekeu, J. Meunier, and E. Rolley, Wetting and Spreading, Rev. Mod. Phys. 81, 739 (2009).

[5] J. Eijkel and A. van den Berg, Nanofluidics: what is it and what can we expect from it?, Microfluid. Nanofluid. 1, 249 (2005).

[6] L. Bocquet and E. Charlaix, Nanofluidics, from bulk to interfaces, Chem. Soc. Rev. 39, 1073 (2010).

[7] V. S. J. Craig, Very small bubbles at surfaces-the nanobubble puzzle, Soft Matter 7, 40 (2011).

[8] J. R. T. Seddon and D. Lohse, Nanobubbles and micropancakes: gaseous domains on immersed substrates, J. Phys.: Condens. Matter 23, 133001 (2011).

[9] J. R. T. Seddon, D. Lohse, W. A. Ducker, and V. S. J. Craig, A Deliberation on Nanobubbles at Surfaces and in Bulk, ChemPhysChem 13, 2179 (2012).

[10] M. P. Brenner and D. Lohse, Dynamic Equilibrium Mechanism for Surface Nanobubble Stabilization, Phys. Rev. Lett. 101, 214505 (2008).

[11] J. W. Gibbs, The Collected Works of J. Willard Gibbs (Yale University Press, London, 1957), p. 288. 
[12] B. Pethica, Contact-Angle Equilibrium, J. Colloid Interface Sci. 62, 567 (1977).

[13] J. Drelich, The significance and magnitude of the line tension in three-phase (solid-liquid-fluid) systems, Colloid Surf. A-Physicochem. Eng. Asp. 116, 43 (1996).

[14] J. Drelich and J. Miller, The Effect of Solid Surface Heterogeneity and Roughness on the Contact Angle/Drop (Bubble) Size Relationship, J. Colloid Interface Sci. 164, 252 (1994).

[15] J. Drelich and J. Miller, The line/pseudo-line tension in three-phase systems, Particul. Sci. Technol. 10, I (1992).

[16] J. Gaydos and A. W. Neumann, The Dependence of Contact Angles on Drop Size and Line Tension, J Colloid Interface Sci. 120, 76 (1987).

[17] D. Li and A. Neumann, Determination of line tension from the drop size dependence of contact angles, Colloids Surfaces 43, 195 (1990).

[18] A. Checco, P. Guenoun, and J. Daillant, Nonlinear dependence of the contact angle of nanodroplets on contact line curvature, Phys. Rev. Lett. 91, 186101 (2003).

[19] G. Lester, Contact angles of liquids at deformable solid surfaces, J. Colloid Sci. 16, 315 (1961).

[20] A. Rusanov, Theory of wetting of elastically deformed bodies. 1. Deformation with a finite contact-angle, Colloid J. USSR 37, 614 (1975).

[21] R. Pericet-Camara, A. Best, H.-J. Butt, and E. Bonaccurso, Effect of capillary pressure and surface tension on the deformation of elastic surfaces by sessile liquid microdrops: An experimental investigation, Langmuir 24, 10565 (2008).

[22] E. R. Jerison, Y. Xu, L. A. Wilen, and E. R. Dufresne, Deformation of an Elastic Substrate by a Three-Phase Contact Line, Phys. Rev. Lett. 106, 186103 (2011).

[23] A. Marchand, S. Das, J. H. Snoeijer, and B. Andreotti, Capillary Pressure and Contact Line Force on a Soft Solid, Phys. Rev. Lett. 108, 094301 (2012). 
[24] R. W. Style, R. Boltyanskiy, Y. Che, J. S. Wettlaufer, L. A. Wilen, and E. R. Dufresne, Universal Deformation of Soft Substrates Near a Contact Line and the Direct Measurement of Solid Surface Stresses, Phys. Rev. Lett. 110, 066103 (2013).

[25] F. Mugele and J. Baret, Electrowetting: From basics to applications, J. Phys.Condes. Matter 17, R705 (2005).

[26] J. Liu, M. Wang, S. Chen, and M. O. Robbins, Uncovering Molecular Mechanisms of Electrowetting and Saturation with Simulations, Phys. Rev. Lett. 108, 098301 (2012).

[27] L. Bocquet and J.-L. Barrat, Flow boundary conditions from nano- to microscales, Soft Matter 3, 685 (2007).

[28] D. M. Huang, C. Sendner, D. Horinek, R. R. Netz, and L. Bocquet, Water Slippage versus Contact Angle: A Quasiuniversal Relationship, Phys. Rev. Lett. 101, 226101 (2008).

[29] C. Sendner, D. Horinek, L. Bocquet, and R. R. Netz, Interfacial Water at Hydrophobic and Hydrophilic Surfaces: Slip, Viscosity, and Diffusion, Langmuir 25, 10768 (2009).

[30] M. Nijmeijer, C. Bruin, A. Bakker, and J. Van Leeuwen, Wetting and drying of an inert wall by a fluid in a molecular-dynamics simulation, Phys. Rev. A 42, 6052 (1990).

[31] J. Kirkwood and F. Buff, The Statistical Mechanical Theory of Surface Tension, J. Chem. Phys. 17, 338 (1949).

[32] J. W. G. Tyrrell and P. Attard, Atomic Force Microscope Images of Nanobubbles on a Hydrophobic Surface and Corresponding Force-Separation Data, Langmuir 18, 160 (2002).

[33] P. Epstein and M. Plesset, On the stability of gas bubbles in liquid-gas solutions, J. Chem. Phys. 18, 1505 (1950).

[34] X. H. Zhang, A. Quinn, and W. A. Ducker, Nanobubbles at the interface between water and a hydrophobic solid, Langmuir 24, 4756 (2008).

[35] E. Karatay, A. S. Haase, C. W. Visser, C. Sun, D. Lohse, P. A. Tsai, and R. G. H. Lammertink, Control of slippage with tunable bubble mattresses, Proc. Natl. Acad. Sci. 110, 8422 (2013). 
[36] X. H. Zhang, G. Li, N. Maeda, and J. Hu, Removal of induced nanobubbles from water/graphite interfaces by partial degassing, Langmuir 22, 9238 (2006).

[37] B. M. Borkent, S. de Beer, F. Mugele, and D. Lohse, On the Shape of Surface Nanobubbles, Langmuir 26, 260 (2010).

[38] B. M. Borkent, S. M. Dammer, H. Schoenherr, G. J. Vancso, and D. Lohse, Superstability of surface nanobubbles, Phys. Rev. Lett. 98, 204502 (2007).

[39] X. H. Zhang, X. Zhang, J. Sun, Z. Zhang, G. Li, H. Fang, X. Xiao, X. Zeng, and $\mathrm{J}$. Hu, Detection of novel gaseous states at the highly oriented pyrolytic graphite-water interface, Langmuir 23, 1778 (2007).

[40] W. A. Ducker, Contact Angle and Stability of Interfacial Nanobubbles, Langmuir 25, 8907 (2009).

[41] X. Zhang, M. H. Uddin, H. Yang, G. Toikka, W. Ducker, and N. Maeda, Effects of Surfactants on the Formation and the Stability of Interfacial Nanobubbles, Langmuir 28, 10471 (2012).

[42] J. R. T. Seddon, H. J. W. Zandvliet, and D. Lohse, Knudsen Gas Provides Nanobubble Stability, Phys. Rev. Lett. 107, 116101 (2011).

[43] S. Das, A. Marchand, B. Andreotti, and J. H. Snoeijer, Elastic deformation due to tangential capillary forces, Phys. Fluids 23, 072006 (2011).

[44] A. Marchand, S. Das, J. H. Snoeijer, and B. Andreotti, Contact Angles on a Soft Solid: From Young's Law to Neumann's Law, Phys. Rev. Lett. 109, 236101 (2012).

[45] R. Shuttleworth, The surface tension of solids, Proc. Phys. Soc., London Sect. A 63, 444 (1950).

[46] P. Muller and A. Saul, Elastic effects on surface physics, Surf. Sci. Rep. 54, 157 (2004).

[47] A. Brotchie and X. H. Zhang, Response of interfacial nanobubbles to ultrasound irradiation, Soft Matter 7, 265 (2011). 


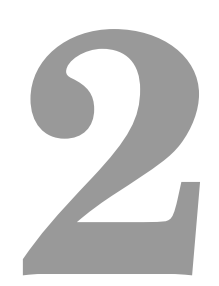

\section{Formation of surface nanobubbles and universality of their contact angles: A molecular dynamics approach *}

We study surface nanobubbles using molecular dynamics simulations of ternary (gas, liquid, solid) systems of Lennard-Jones fluids. They form for a sufficiently low gas solubility in the liquid, i.e., for a large relative gas concentration. For a strong enough gas-solid attraction, the surface nanobubble is sitting on a gas layer, which forms in between the liquid and the solid. This gas layer is the reason for the universality of the contact angle, which we calculate from the microscopic parameters. Under the present equilibrium conditions the nanobubbles dissolve within less of a microsecond, consistent with the view that the experimentally found nanobubbles are stabilized by a nonequilibrium mechanism.

\subsection{Introduction}

When liquid comes into contact with a solid, nanoscopic gaseous bubbles can form at the interface: surface nanobubbles [1-3]. These bubbles were discovered about 15 years ago, after Parker et al. predicted their existence to ex-

\footnotetext{
*Published as: J.H. Weijs, J.H. Snoeijer, and D. Lohse, Phys. Rev. Lett. 108, 104501 (2012).
} 
plain the long-ranged attraction between hydrophobic surfaces in water [4]. Many atomic force microscopy (AFM) and spectroscopy measurements have since then confirmed the existence of spherical cap-shaped, gaseous bubbles at the liquid-solid interface.

Various open questions remain about surface nanobubbles, and in this chapter we will address three crucial ones: (i) How do surface nanobubbles form? This question is difficult to answer by experimental means, since the formation process is too fast to be observed by AFM. (ii) A second question regards the contact angle of surface nanobubbles which is found to disagree with Young's law: all recorded nanobubbles have a much lower gas-side contact angle than expected, and seem to be universal within 20 degrees. (iii) Finally, AFM showed that surface nanobubbles can be stable for hours or even days, whereas the pressure inside these bubbles due to their small radius of curvature $\left(R_{c} \sim 100 \mathrm{~nm}\right)$ would be several atmospheres due to the Laplace pressure: $\Delta p=2 \gamma / R_{c}$, with $\gamma$ the liquid-vapour surface tension. A simple calculation then shows that surface nanobubbles should dissolve within microseconds, which is 9 to 10 orders of magnitudes off with respect to the experimental data.

In this chapter, we use molecular dynamics (MD) simulations to study surface nanobubbles in simple fluids. Using MD simulations, we are able to answer questions (i) and (ii), and provide important information with respect to question (iii). MD simulations are well-suited for nanobubbles, because the temporal resolution is of order fs, and since all atom's motions are resolved, the spatial resolution is intrinsically high enough to resolve nanobubbles. This atomistic model allows us to study microscopic details that are inaccessible by experimental means and standard continuum mechanics. Figure 2.1 shows how surface nanobubbles form in a typical simulation of a liquid containing gas. The gas will homogeneously nucleate to form a bubble, which subsequently attaches to the wall. We will analyze the nucleation process in detail and quantify how the contact angle of the bubble changes upon varying gas solubility. The enhanced gas concentration ("gas-enrichment layer") at the solid-liquid interface, which is strongest at hydrophobic substrates, will turn out to play a key role to account for the universality of the contact angle. 

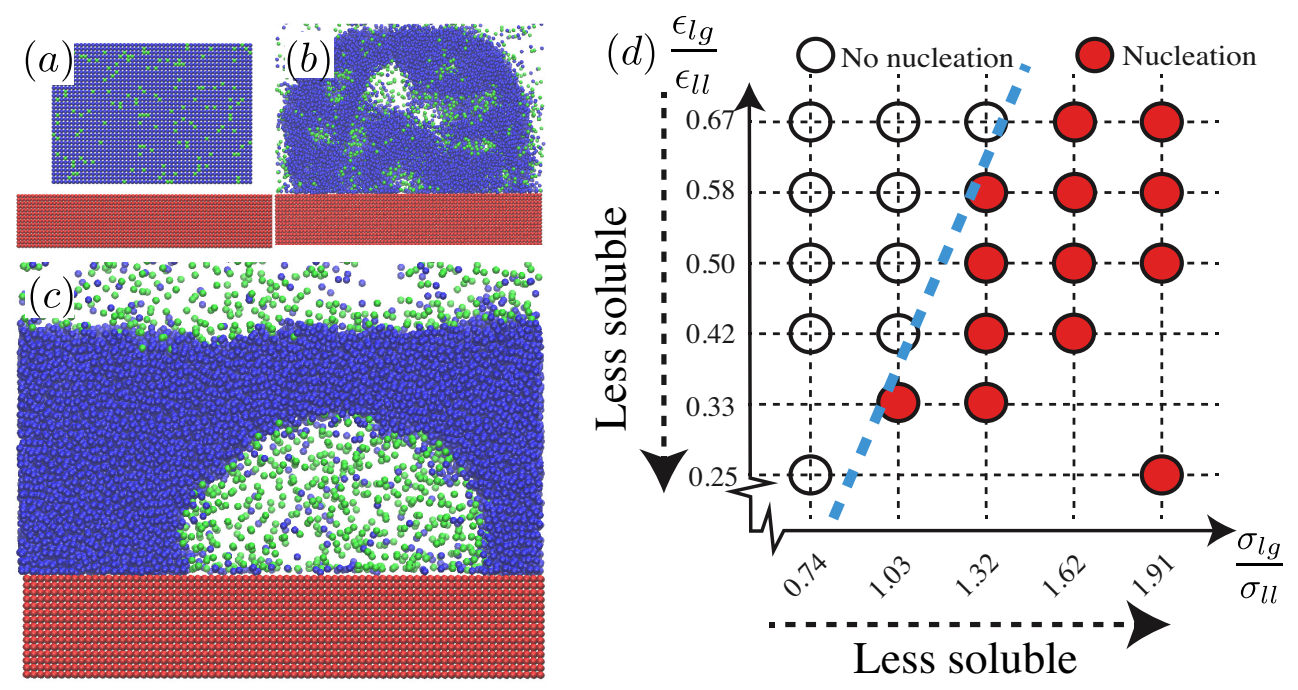

Figure 2.1: (a) Initial conditions: a liquid layer (blue) is placed on top of a solid substrate (bottom, red). Gas is dissolved inside the liquid layer (green). (b) Simulation $\left(\epsilon_{l g} / \epsilon_{l l}=0.58, \sigma_{l g} / \sigma_{l l}=1.32\right)$ after about 0.1 ns: nucleation occurs. (c) $t=10$ ns: a surface nanobubble has formed. (d) Parameter space where the solubility of the gas was tuned through the parameters $\epsilon_{l g} / \epsilon_{l l}$ and $\sigma_{l g} / \sigma_{l l}$. As the gas becomes increasingly soluble [going up, left in Fig. 2.I(c)] a sudden transition takes place where no nanobubbles nucleate. The gas then remains in a dissolved state and partially escapes to the gas-phase above the liquid layer until equilibrium is reached.

\subsection{Numerical setup}

The studies in this chapter are performed using simple fluids, which contain no molecules but rather separate atoms that interact with each other through the Lennard-Jones (LJ12-6) potential:

$$
U=4 \epsilon_{i j}\left[\left(\frac{\sigma_{i j}}{r}\right)^{12}-\left(\frac{\sigma_{i j}}{r}\right)^{6}\right] .
$$

Here, $\epsilon_{i j}$ and $\sigma_{i j}$ are the interaction strength and range between particles $i$ and $j$, respectively. All simulations were performed using the Gromacs software package and were done at constant temperature, volume, and number of particles $(T, V$, and $N$ constant). The augmented Berendsen thermostat described in ref. [5] was used in all simulations. We verified that this thermostat yields the same result as the Nosé-Hoover thermostat. In all 
simulations three types of particles were used: fluid I, fluid II, and solid particles. The fluid particles (I \& II) behave like ordinary particles in a MDsimulation and thus move around the system. Contrarily, the solid particles are constrained to their initial position throughout time and constitute the immobile substrate. The interaction parameters of the fluids are chosen such that at the temperature considered $(T=300 \mathrm{~K})$ fluid $\mathrm{I}$ is in the liquid state and fluid II in the gas state, and they will be referred to by these states throughout the rest of the chapter. These interaction parameters are: $\left(\sigma_{s s}, \sigma_{l l}, \sigma_{g g}\right)=(0.34,0.34,0.50) \mathrm{nm},\left(\epsilon_{s s}, \epsilon_{l l}, \epsilon_{g g}\right)=(1.2,1.2,0.4) k_{B} T$, with $k_{B}$ Boltzmann's constant. For cross interactions we use: $\sigma_{i j}=\left(\sigma_{i i}+\sigma_{j j}\right) / 2$, and $\left(\epsilon_{s l}, \epsilon_{s g}, \epsilon_{l g}\right)=(0.8,0.8,0.7) k_{B} T$, unless otherwise stated in the text. The cut-off length of the potential function was set at $r_{c}=5 \sigma=1.7 \mathrm{~nm}$.

The time step for the simulations is $d t=\tau / 400 \approx 2 \mathrm{fs}$, where $\tau$ is a characteristic timescale of atomic motion $\tau=\sigma_{l l} \sqrt{m / \epsilon_{l l}}$, with $m$ the mass of the liquid and gas particles (20 amu). The initial conditions are shown in Fig. 2.1a: on top of the substrate we place a layer of liquid with dissolved gas. Periodic boundary conditions are present in all directions $(x, y, z)$; the resulting nanobubbles are approximately $20-30 \mathrm{~nm}$ wide, and $10-20 \mathrm{~nm}$ high, depending on the contact angle. The formation and behavior of the bubbles was found to be independent of simulation box-size, which was set at $40 \times 40 \times 5.5 \mathrm{~nm}^{3}$.

\subsection{Bubble nucleation}

What determines whether nanobubbles form? We address this question by varying the relative interaction strength and the relative interaction size. We then explore the parameter space to see under what conditions nanobubbles form. The result is shown in Fig. 2.1d. Decreasing $\epsilon_{l g} / \epsilon_{l l}$ (going down in Fig. 2.1d) results in a lower solubility of gas in the liquid, and since the absolute concentration of gas is kept constant this means that the liquid becomes more and more supersaturated. Eventually, homogeneous nucleation occurs and a nanobubble forms in the bulk liquid phase, which finally attaches to the surface. Increasing $\sigma_{l g} / \sigma_{l l}$ [going right in Fig. 2.1(d)] leads to the same effect: due to the increased size of the gas atoms it becomes energetically less favorable to remain dissolved in the liquid phase. Eventually, when the gas molecules are large enough nanobubbles form due to the supersaturation of gas in the liquid. From this, we can conclude that a local supersaturation of gas inside the liquid is a possible mechanism to generate surface nanobub- 


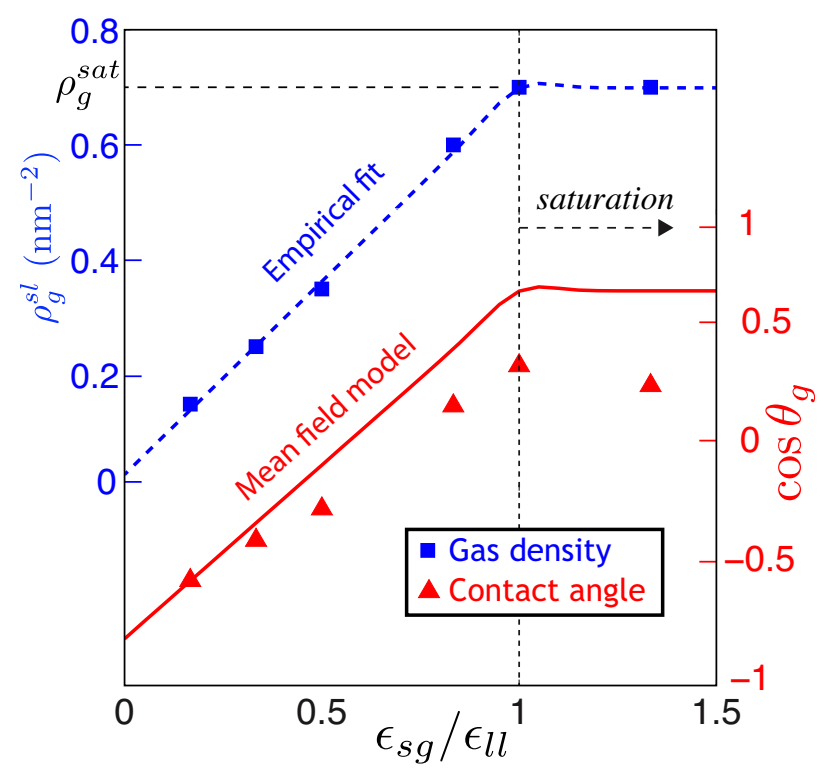

Figure 2.2: The effect of an enhanced gas-solid interaction strength. As the interaction strength $\epsilon_{s g} / \epsilon_{l l}$ is increased, the adsorbed gas density ( $\rho_{g}^{s l}$, blue squares) increases as well until a saturation limit. As the adsorbed gas density increases, the gas-side contact angle $\theta$ lowers ( $\cos \theta$ indicated by red triangles). The red solid line is a fit to the mean-field expression (2.2) taking into account the screening of the adsorbed gas (see text).

bles. These results are consistent with the experimental findings in ref. [6], where it was reported that nanobubble formation strongly depends on the (relative) gas concentration in the liquid. Although the concentration required to spontaneously form nanobubbles is far greater than the saturation concentration, we point out that during deposition of liquid on a substrate gas can be trapped leading to very high local transient concentrations which would not be reflected in measurements of the global gas concentration in the liquid. In fact, numerous experimental papers have pointed out that the method of deposition is of great importance for achieving surface nanobubbles [7]. We have to note, however, that other mechanisms not considered here can also induce the formation of nanobubbles (e.g. heterogeneous nucleation, bulk desorption of gas from micropancakes $[6,8])$. The nanobubbles produced in our simulations are found to be reproducible and, at the very least, can be studied regarding their shape and stability. 


\subsection{Universal contact angle}

Now that we can simulate nanobubbles, we focus on the universal contact angle of surface nanobubbles found in experiments [9-11]. For this we use similar initial conditions as in Fig. 2.1a, with a region in the liquid with a very high gas concentration, providing us with control over where the nanobubbles form, and how much gas they contain (about 1,00o atoms). We measure the gas-side contact angle ${ }^{\dagger}$ at varying values of the solid-gas interactions $\epsilon_{s g}$. As can be seen in Fig. 2.2 (triangles), we observe that the gas-side contact angle of the nanobubble decreases (i.e. the nanobubble becomes flatter), as the solid-gas interaction is increased. This trend saturates around $\epsilon_{s g} / \epsilon_{l l} \approx 1$, where the gas-solid attraction matched the liquid-liquid attraction $¥$. The observed saturation contact angle $\left(75^{\circ}\right)$ is close to the contact angle of nanobubbles that is found in experiments $\left(60^{\circ}\right)$. On the same figure we show the evolution of the $2 \mathrm{D}$ number density of gas concentration at the wall inside the liquid phase, $\rho_{g}^{s l}$. Remarkably, this concentration exhibits a trend that is very similar to that of the contact angle. Stronger solid-gas interactions lead to a planar area of high gas concentration at the solid-liquid interface, which is called a gas-enrichment layer and which has been observed to exist in both simple liquids as well as real liquids [13-15]. In experiments high-density gas adsorbates (micropancakes) have also been observed [6, 8]. Figure 2.2 shows that the adsorbate density increases with $\epsilon_{s g}$, until it finally saturates to a $2 \mathrm{D}$ number density of $\rho_{g}^{s l}=0.7$ atoms per $\mathrm{nm}^{2}$ in the first gas layer above the substrate.

The increase of the gas density near the wall is indeed the origin of the flattening of the nanobubbles. Namely, the presence of the gas weakens the attractive interaction between solid and liquid molecules: The liquid does not "feel" a solid half-space anymore, but there is now a dense gas-layer that effectively renders the wall more hydrophobic. This effect can be quantified using the approximate equation for the contact angle [16-18],

$$
\cos \theta_{g}=1-2 \frac{\rho_{s} \epsilon_{s l}}{\rho_{l} \epsilon_{l l}}
$$

which can be obtained from a mean-field argument. This expression contains only the solid and liquid densities $\rho_{s}, \rho_{l}$, and the solid-liquid and

\footnotetext{
${ }^{\dagger}$ Similarly as done in chapter 7 for nanodroplets.

$\ddagger$ A realistic value for the energy between gaseous argon and individual carbon atoms in graphene is: $\epsilon_{A r-C}=0.2 k_{b} T$. For the energies between the oxygens of two water molecules: $\epsilon_{O_{l}-O_{l}}=0.26 k_{b} T[12]$.
} 
liquid-liquid interactions $\epsilon_{s l}, \epsilon_{l l}$. The vapor phase has a negligible contribution $\left(\rho_{v}\right.$ is small compared to $\rho_{l}$ and $\rho_{s}$ ) and the solid-solid interaction is irrelevant since the solid is nondeformable. In the case of a dense adsorbate, the attraction $\epsilon_{s l}$ is reduced to an effective interaction $\tilde{\epsilon}_{s l}$, with $\tilde{\epsilon}_{s l}<\epsilon_{s l}$. In addition, the adsorbate density is lower than the original solid density, due to the large size of the gas atoms, and gives an 'effective' density $\tilde{\rho}_{s}<\rho_{s}$. According to (2.2), both effects lead to a lower gas-side contact angle. The solid line in Fig. 2.2 shows the predicted contact angle by this expression, assuming an average interaction strength: $\tilde{\epsilon}_{s l}=\left(\epsilon_{s l}+\epsilon_{l g}\right) / 2$. Here the effective density is estimated by $\tilde{\rho}_{s}=\left(1-\frac{\rho_{g}^{s l}}{\rho_{g}^{\text {sat }}}\right) \rho_{s}+\rho_{g}$, as a phenomenological description for the screening of the solid as the adsorbate layer density grows. Note that the influence of the vapor phase is neglected, as was the case in the model without the presence of a gas adsorbate. The model quantitatively explains the observations in MD simulations, in particular, the saturation of the contact angle occurs exactly when a complete layer of gas adsorbate is formed. It therefore provides a very natural explanation for the observed universal contact angles in experiments. [9-11, 19]

\subsection{Stability}

Another aspect of nanobubbles that can now be studied is their stability. Are Lennard-Jones nanobubbles stable? After formation of the nanobubble, we use a shape tracker to follow the dynamics of the nanobubble. The shape tracker locates a nanobubble by performing a circular fit through the liquidvapour interface of the curved bubble wall. Different quantities can then be computed, such as the radius of curvature, the contact angle, the amount of gas inside a nanobubble, and the angular dependence of gas flux through the bubble wall. A good indicator for nanobubble stability is the gas content inside the bubble: when the amount of gas remains constant the bubble is considered stable. The gas contents of nanobubbles on different substrates as a function of time are plotted in Fig. 2.3a. We see that none of the nanobubbles are stable; they dissolve on a timescale $(\mu \mathrm{s})$ much shorter than that observed in experiments (days), see also the inset in Fig. 2.3. However, this fast decay is in agreement with simple macroscopic diffusion calculations. Furthermore, we find that the contact angle of the nanobubbles does not change significantly throughout the dissolution process.

Although the nanobubbles are not stable, some interesting events oc- 


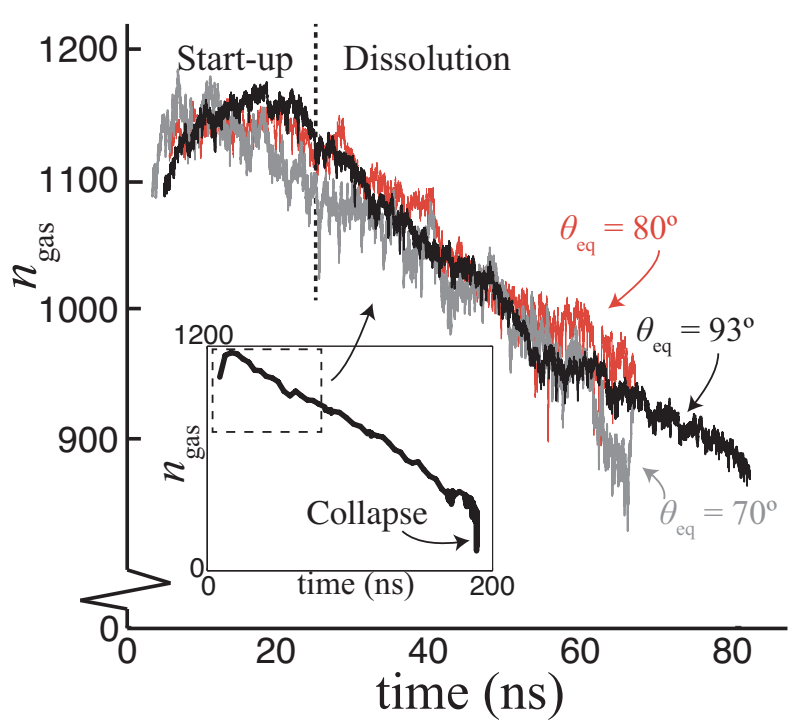

Figure 2.3: Number of gas molecules $n_{\text {gas }}$ inside the nanobubble as a function of time for nanobubbles on different substrates with different equilibrium contact angles. Initially, the fluid is supersaturated and a bubble quickly forms within a few ns. Shortly after the bubble has formed, the liquid is still supersaturated causing gas to enter the bubble ("Start-up"). After about 20 ns the gas in the liquid achieves the equilibrium concentration, and the nanobubbles start to dissolve ("Dissolution"). The dissolution rate of the nanobubbles is independent of the contact angle. The inset shows the full dissolution of the $\theta_{e q}=93^{\circ}$ bubble after $0.2 \mu \mathrm{s}$.

cur near the contact line. When studying the time-averaged local flux as a function of angular position $\phi$ (Fig. 2.4b) we see that the flux is highest near the contact line, indicating that the substrate plays an important role in nanobubble stability. This strong localized flux near the contact line is heavily influenced by the presence and strength of the gas-enrichment layer, which is a plane at the solid-liquid interface in which gas atoms can move relatively easily due to a liquid depletion layer that exists at the same position. The influx indicates that there may exist a condition where a dynamic equilibrium is achieved, i.e., the diffusive outflux is balanced by the influx at the contact line, explaining why in nonequilibrium surface nanobubbles can be dynamically stable [20] (and bulk nanobubbles cannot). A coarse exploration of the parameters $\epsilon_{s l}$ and $\epsilon_{s g}$ has been performed in this study, but under the present equilibrium conditions a stable nanobubble was not 


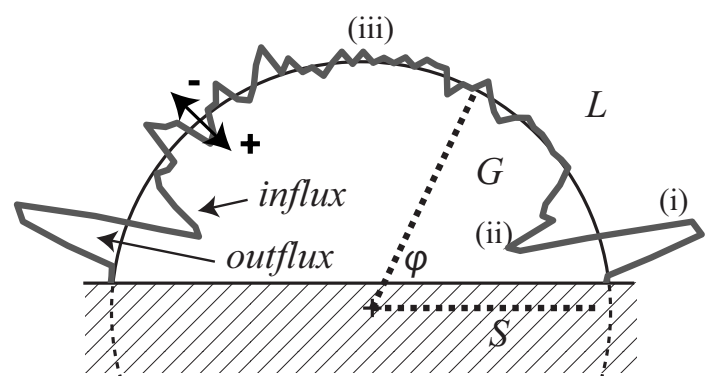

Figure 2.4: Local flux of gas through the liquid-vapour interface of a nanobubble attached to a substrate. The gray line indicates the time-averaged local gas flow direction and strength. The gas flow is directed outwards everywhere (iii), except for a small region near the contact line [(i) and (ii)] where in a very small region a strong in- and outflux are observed, indicating that there exists a recirculation current. The net effect of this recirculation current is found to be of the same order as the diffusive outflux.

achieved.

Of course, there are many more parameters that need to be explored, such as the initial radius of the bubble: it is possible that nanobubbles below a certain critical size are unstable. Also, Lennard-Jones fluids might not contain the necessary properties to form stable nanobubbles, such as electrostatic effects. Most importantly, for the dynamic equilibrium theory to be true, some driving force must exist to sustain the circulation of gas. This means that the equilibrium simulations in this study need to be adapted to contain such a driving force. Such non-equilibrium effects include the presence of a thermal gradient (which are likely to be present in experimental setups as well) or the formation of gas at the substrate (which has been studied using electrolysis [21-23]).

\subsection{Outlook}

In conclusion, we have generated and analysed the formation and stability of surface nanobubbles in simple fluids. We found that in heavily gassupersaturated liquids nanobubbles nucleate spontaneously which can then migrate towards the surface. In experiments, when water is deposited on the substrate, it is possible that some gas becomes trapped near the solidliquid interface leading to the required supersaturation. Other formation 
mechanisms that cannot be accessed by MD simulations can however not be excluded, further work is required on this question. The universal contact angles that surface nanobubbles exhibit in experiments can be explained by a dense layer of gas at the solid-liquid interface, which has been shown to exist for real liquids, that effectively alters the substrate chemistry. Although the Lennard-Jones nanobubbles are unstable, some interesting local gas flows are present near the contact line. These gas flows are caused by the symmetry breaking due to the solid substrate, and hint towards a dynamic equilibrium condition where the diffusive outflux is compensated by an influx near the contact line. Since an energy input is required to sustain a circulatory gas flow as suggested in the dynamic equilibrium theory by Brenner and Lohse [20], it is likely that stable nanobubbles can only occur in non-equilibrium systems. Simulations of non-equilibrium systems, and of systems containing realistic fluids must be performed to address the question regarding the long lifetime of surface nanobubbles.

\section{References}

[1] M. A. Hampton and A. V. Nguyen, Nanobubbles and the nanobubble bridging capillary force, Adv. Colloid Interface Sci. 154, 30 (2010).

[2] J. R. T. Seddon and D. Lohse, Nanobubbles and micropancakes: gaseous domains on immersed substrates, J. Phys.: Condens. Matter 23, (2011).

[3] V. S. J. Craig, Very small bubbles at surfaces-the nanobubble puzzle, Soft Matter 7, 40 (2011).

[4] J. Parker, P. Claesson, and P. Attard, Bubbles, cavities, and the long-ranged attraction between hydrophobic surfaces, J. Phys. Chem. 98, 8468 (1994).

[5] G. Bussi, D. Donadio, and M. Parrinello, Canonical sampling through velocity rescaling, J. Chem. Phys. 126, 014101 (2007).

[6] J. R. T. Seddon, E. S. Kooij, B. Poelsema, H. J. W. Zandvliet, and D. Lohse, Surface Bubble Nucleation Stability, Phys. Rev. Lett. 106, 056101 (2011).

[7] X. H. Zhang, X. D. Zhang, S. T. Lou, Z. X. Zhang, J. L. Sun, and J. Hu, Degassing and Temperature Effects on the Formation of Nanobubbles at the Mica/Water Interface, Langmuir 20, 3813 (2004). 
[8] X. H. Zhang, X. Zhang, J. Sun, Z. Zhang, G. Li, H. Fang, X. Xiao, X. Zeng, and J. Hu, Detection of Novel Gaseous States at the Highly Oriented Pyrolytic Graphite-Water Interface, Langmuir 23, 1778 (2007).

[9] N. Ishida, T. Inoue, M. Miyahara, and K. Higashitani, Nano Bubbles on a Hydrophobic Surface in Water Observed by Tapping-Mode Atomic Force Microscopy, Langmuir 16, 6377 (2000).

[10] J. Yang, J. Duan, D. Fornasiero, and J. Ralston, Very Small Bubble Formation at the Solid-Water Interface, The Journal of Physical Chemistry B 107, 6139 (2003).

[11] X. H. Zhang, N. Maeda, and V. S. J. Craig, Physical Properties of Nanobubbles on Hydrophobic Surfaces in Water and Aqueous Solutions, Langmuir 22, 5025 (2006).

[12] C. Oostenbrink, A. Villa, A. E. Mark, and W. F. Van Gunsteren, $A$ biomolecular force field based on the free enthalpy of hydration and solvation: The GROMOS force-field parameter sets $53 A_{5}$ and $53 A 6$, J. Comp. Chem. 25, 1656 (2004).

[13] S. M. Dammer and D. Lohse, Gas Enrichment at Liquid-Wall Interfaces, Phys. Rev. Lett. 96, 206101 (2006).

[14] D. Bratko and A. Luzar, Attractive Surface Force in the Presence of Dissolved Gas: A Molecular Approach, Langmuir 24, 1247 (2008).

[15] C. Sendner, D. Horinek, L. Bocquet, and R. R. Netz, Interfacial Water at Hydrophobic and Hydrophilic Surfaces: Slip, Viscosity, and Diffusion, Langmuir 25, 10768 (2009).

[16] J. S. Rowlinson and B. Widom, Molecular Theory of Capillarity (Dover publications, Mineola, NY, 1982).

[17] C. Bauer and S. Dietrich, Quantitative study of laterally inhomogeneous wetting films, Eur. Phys. J. B 10, 767 (1999).

[18] J. H. Snoeijer and B. Andreotti, A microscopic view on contact angle selection, Phys. Fluids 20, 057101 (2008).

[19] M. A. J. van Limbeek and J. R. T. Seddon, Surface Nanobubbles as a Function of Gas Type, Langmuir 27, 8694 (2011). 
[20] M. P. Brenner and D. Lohse, Dynamic Equilibrium Mechanism for Surface Nanobubble Stabilization, Phys. Rev. Lett. 101, 214505 (2008).

[21] L. Zhang, Y. Zhang, X. Zhang, Z. Li, G. Shen, M. Ye, C. Fan, H. Fang, and J. Hu, Electrochemically Controlled Formation and Growth of Hydrogen Nanobubbles, Langmuir 22, 8109 (2006).

[22] F. Hui, B. Li, P. He, J. Hu, and Y. Fang, Electrochemical fabrication of nanoporous polypyrrole film on HOPG using nanobubbles as templates, Electrochemistry Communications 11, 639 (2009).

[23] S. Yang, P. Tsai, E. S. Kooij, A. Prosperetti, H. J. W. Zandvliet, and D. Lohse, Electrolytically Generated Nanobubbles on Highly Orientated Pyrolytic Graphite Surfaces, Langmuir 25, 1466 (2009). 


\section{Diffusive shielding stabilizes bulk nanobubble clusters *}

Using molecular dynamics, we study the nucleation and stability of bulk nanobubble clusters. We study the formation, growth, and final size of bulk nanobubbles. We find that, as long as the bubble-bubble interspacing is small enough, bulk nanobubbles are stable against dissolution. Simple diffusion calculations provide an excellent match with the simulation results, giving insight into the reason for the stability: nanobubbles in a cluster of bulk nanobubbles "protect" each other from diffusion by a shielding effect.

\subsection{Introduction}

Gas bubbles are ubiquitous in nature, industry and daily life. They are found in streams of water, manufacturing processes of many types of materials, and, of course, when we enjoy a carbonated drink. Even though gas bubbles are commonly present in many of the liquids we deal with on a daily basis, bubbles are, in fact, usually unstable against dissolution in the medium that surrounds them [1]. The dissolution rate increases as the bubble becomes

*Published as: J.H. Weijs, J.R.T. Seddon, and D. Lohse, “Diffusive shielding stabilizes bulk nanobubble clusters", Chem. Phys. Chem 13, 2197-2204 (2012). 
smaller because of the increased (Laplace) pressure $\Delta p=2 \gamma / R$ inside the bubble, where $\gamma$ is the interfacial tension of the bubble wall and $R$ the bubble radius. The consequence is that nanoscopic bubbles cannot survive for more than a few microseconds.

In contrast to this expectation, surprisingly, experiments by Ohgaki et al. [2] have shown that stable bulk nanobubbles do exist, although there is some discussion on whether these are actually nanobubbles rather than impurities $[3,4]$. In these experiments the bubbles were observed to be packed closely together (the distance between neighbouring bubbles was measured to be less than 10R), suggesting that a shielding mechanism between bubbles may act to keep the bubbles from dissolving. In addition to this direct observation of bulk nanobubbles, their presence has also been indirectly measured in experiments, using dynamic laser light scattering $[5,6]$. Although this technique cannot distinguish between nanobubbles and liquid density variations in the liquid caused by other sources (such as large organic molecules), the observed fluctuations disappear after degassing the liquid, indicating that the observed objects are indeed bulk nanobubbles.

In addition to these experiments, there are many publications where the presence of surface nanobubbles are observed at liquid-solid interfaces. Generally, these surface nanobubbles are detected by Atomic Force Microscopy (AFM), and they can survive for days [7-9]. Similar to bulk nanobubbles, surface nanobubbles should dissolve within microseconds, in contrast to the AFM observations $[7,8,10-19]$. Various stabilization mechanisms have been proposed $[7-9,20,21]$, and many of them invoke the direct bubble-wall interaction. This in particular holds for the dynamic equilibrium theory promoted by some of us $[9,21,22]$. This stabilization mechanisms is therefore not applicable to bulk nanobubbles: the symmetry breaking caused by the presence of the substrate in the case of surface nanobubbles does not exist for bulk nanobubbles.

On the other hand, different stabilization mechanisms may exist that could account for stable bulk nanobubbles. Such a mechanism will be discussed in this chapter: when a bulk nanobubble is surrounded by more nanobubbles, the diffusive outflux is 'shielded': a locally high concentration of dissolved gas in the water suppresses the diffusive outflux from the bubble. For this to happen a cluster of bubbles must exist where the spacing between bubbles is not too large. Indeed, the bulk nanobubbles reported by Ohgaki et al. [2] have a distance of $10 R$ or less.

Previously, some molecular dynamics (MD) simulations studies were 


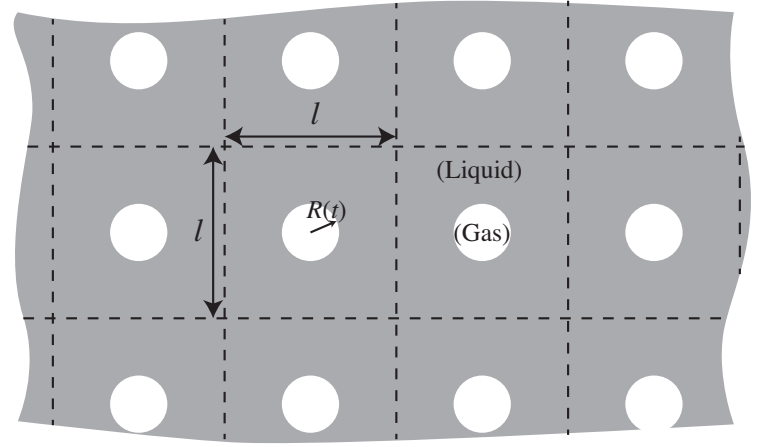

Figure 3.1: A cluster of nanobubbles on a rectangular grid. The dotted lines indicate the unit cell, which is rectangular and has sides with length $l$ : the distance between two neighbouring bubbles.

performed on nanobubbles, where it was found that surface tension plays a role for bubbles larger than $1 \mathrm{~nm}[23]$. In an earlier study it was found that gas concentration is a crucial parameter for the formation of surface nanobubbles [24]. To our knowledge no systemetic study of the stability of bulk nanobubbles has been performed using MD.

In this chapter, we will discuss MD simulations of binary mixtures of simple (Lennard-Jones) fluids. One of the fluids is under the imposed conditions $\left(T=300 \mathrm{~K}, p=10^{5} \mathrm{~Pa}\right)$ in the liquid state, the other in the gaseous state. The simulations will be carried out in a simulation domain of which one dimension is very small $(\ell \times \ell \times d, d \ll \ell)$, such that the simulations are quasi-2D, see also Fig. 3.1. For a full $3 \mathrm{D}$ case, the results will only differ quantitatively, but qualitatively they will be the same. Periodic boundary conditions are applied in all directions, such that we only have to simulate one single nanobubble which is then mirrored. This infinite repetition of nanobubbles then represents an infinite (periodic) nanobubble cluster ( see Fig. 3.1) in a closed system. The closed system means that the total amount of gas is conserved. In this chapter, we explore two box sizes: $\ell=15 \mathrm{~nm}$ and $\ell=30 \mathrm{~nm}$, with $d=3.64 \mathrm{~nm}$ in both cases. Due to the periodic boundary conditions the distance between neighbouring bubbles is $\ell$.

The chapter is organized as follows: In section 3.2 the numerical details of the simulations will be outlined, such as the parameters and algorithms used, as well as the initial conditions. Next, in section 3.3, the results of the MD-simulations will be presented and discussed, and in section 3.4 we 
will compare these results (particularly the equilibrium radius $R_{e q}$ of bulk nanobubbles) with continuum predictions. Finally, in section 3.5 we will discuss the stability of the entire cluster (as opposed to just single bubbles inside the cluster).

\subsection{Numerical details}

\subsubsection{Molecular Dynamics}

To simulate a cluster of bulk nanobubbles, we use Molecular Dynamics (MD) simulations of simple fluids. The atoms in the simple fluids interact with each other through the Lennard-Jones potential:

$$
U_{L J}(r)=4 \epsilon_{i j}\left[\left(\frac{\sigma_{i j}}{r}\right)^{12}-\left(\frac{\sigma_{i j}}{r}\right)^{6}\right] .
$$

Here, $\epsilon_{i j}$ is the interaction strength between atom species $i$ and $j$, and $\sigma_{i j}$ the interaction radius between atoms species $i$ and $j$. In our simulations we use two atom types: the first is in the liquid state under the conditions considered ( $\left.p=10^{5} \mathrm{~Pa}, T=300 \mathrm{~K}\right)$ and the second in the gas state. The interactions are defined as follows: $\left(\epsilon_{l l}, \epsilon_{g g}, \epsilon_{l g}\right)=\left(3,1, \sqrt{\epsilon_{l l} \epsilon_{g g}}=1.73\right) \mathrm{kJ} / \mathrm{mol}$, $\left(\sigma_{l l}, \sigma_{g g}, \sigma_{l g}\right)=\left(0.34,0.5,\left(\sigma_{l l}+\sigma_{g g}\right) / 2=0.42\right) \mathrm{nm}$. The simulations are carried out in the NPT-ensemble (constant number of particles, pressure, and temperature). A Berendsen pressure scaling algorithm was applied, and the temperature was kept constant using the thermostat described in ref. [25].

\subsubsection{Initial conditions}

We use two different atom start-position configurations, which are shown in Fig. 3.2. The first configuration consists of a preformed bubble at a predefined radius $R_{0}$ containing gas ( 333 atoms) and vapour. Outside the bubble the simulation box is completely filled with liquid, and the remainder of the gas is uniformly dissolved throughout the liquid. For the second configuration, the simulation box is completely filled with liquid with the gas uniformly dissolved in this liquid (so no pre-existing bubble). In this configuration, a nanobubble will occur if the concentration of gas in the liquid is high enough such that the energy barrier for homogeneous nucleation can be overcome. Since the pressure is maintained constant throughout the simulation, the box-size is allowed to vary to accommodate this. In practice, we find 

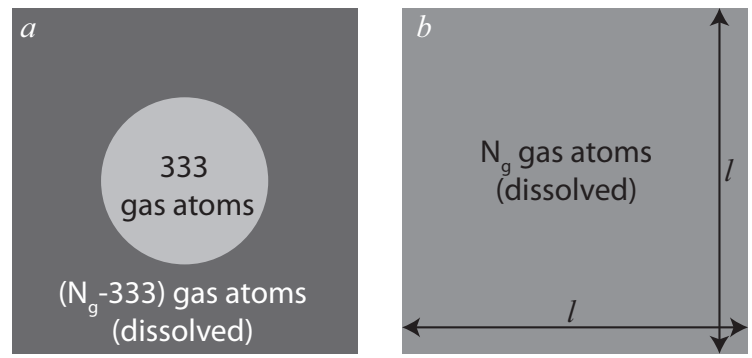

Figure 3.2: The two types of initial conditions for the simulations. a) A preformed bubble containing 333 gas-atoms surrounded by liquid. If there are more gas atoms in the system $\left(N_{g}>333\right)$ they are uniformly dissolved throughout the liquid. b) All $\left(N_{g}\right)$ gas atoms are uniformly dissolved throughout the liquid, so there is no preformed bubble. If the concentration of gas is high enough, homogeneous nucleation will occur forming a nanobubble.

that the box dimensions never vary more than 10\% from their initial values. The initial velocities for all atoms are sampled from a Maxwell-Boltzmann distribution at $300 \mathrm{~K}$.

\subsection{Results from the MD simulations}

A total of 8 different bubbles have been simulated (see Table 3.1) which started with a pre-existing bubble in the initial conditions (Fig. 3.2a). Of those configurations 5 additional simulations were performed using the initial conditions without a pre-existing bubble (Fig. 3.2b), to see whether the initial conditions affect the final result. The boundary of the bubble is defined at $\rho^{*}=0.5$, where:

$$
\rho^{*}(\vec{r})=\frac{\rho(\vec{r})-\rho_{v}}{\rho_{l}-\rho_{v}} .
$$

Here, $\rho_{v}$ is the bulk number density of the gas/vapour phase inside the bubble and $\rho_{l}$ the number density in the bulk liquid. This boundary is then fitted with a circle giving $R(t)$. Some snapshots of a selection of simulations are shown in Fig. 3.3. $R(t)$ against time is plotted in Fig. 3.4 where we see that some bubbles are stable, while others are not.

As one would intuitively expect, the bubbles that are closest together $(\ell=15 \mathrm{~nm}$, configurations I-IV) are stable, whereas some bubbles that are spaced further apart $(\ell=30 \mathrm{~nm})$ are not. The stable bubbles benefit from 


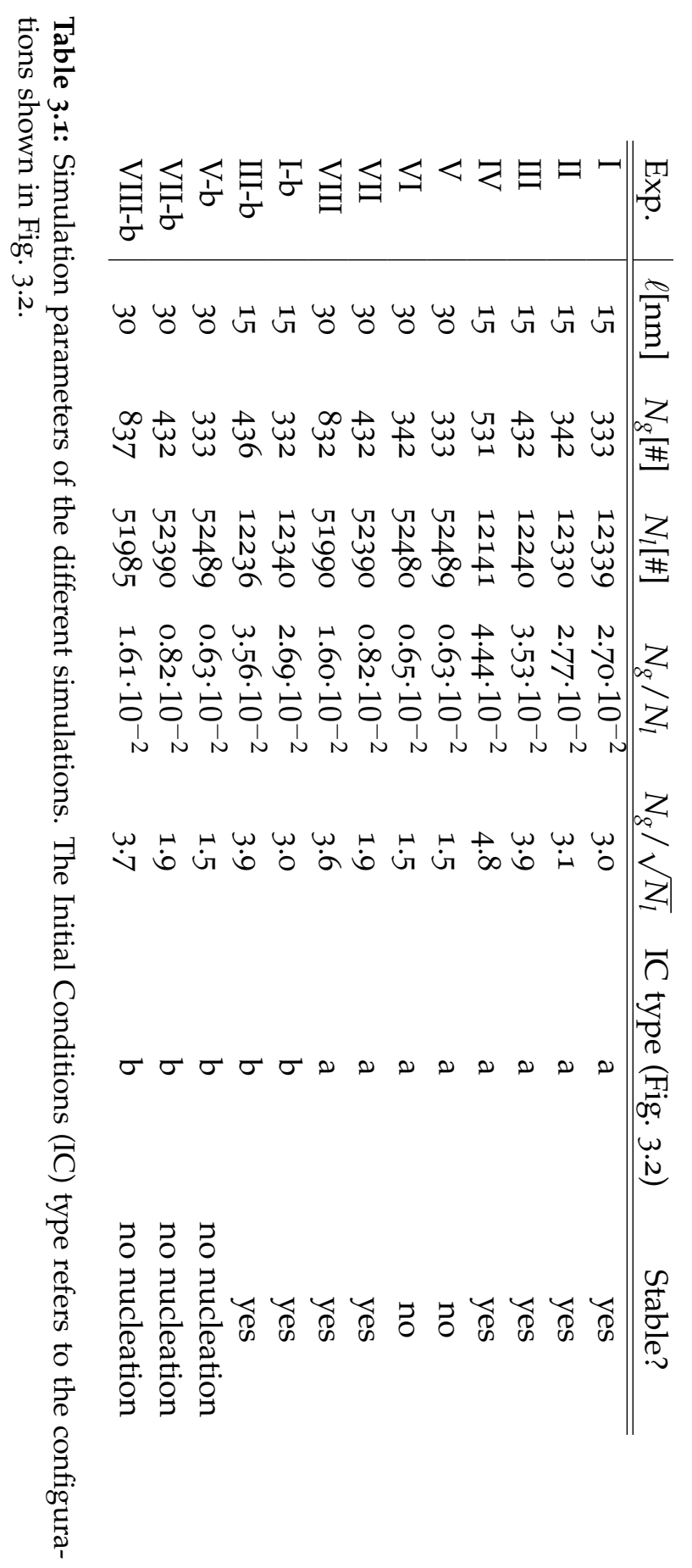


their nearest neighbours, as they 'shield' the diffusive outflux that would normally lead to dissolution within microseconds. To confirm that these bubbles are truly stable, we extended one simulation (configuration III) until $t=0.8 \mu \mathrm{s}$, where we found that after $t=3 \mathrm{~ns}$ the radius $R$ remained perfectly constant (see inset Fig. 3.4).

The bubbles that are spaced farther from each other $(\ell=30 \mathrm{~nm}$, configurations V-VIII) are not always stable. The configurations with the least amount of gas (configurations $\mathrm{V}$ and $\mathrm{VI}$ ) dissolve within $70 \mathrm{~ns}$, whereas configurations VII and VIII - which contain more gas - are stable.

What happens when the initial conditions are changed from a pre-existing bubble (Fig. 3.2a) to uniformly dissolved gas in bulk liquid (Fig. 3.2b)? If a bubble forms, there is no reason why it shouldn't grow to the same equilibrium size as the corresponding bubbles with different initial conditions. In fig. 3.5 we show the results of some simulations with the alternative initial conditions (I-b, III-b, V-b, VII-b, and VIII-b) compared to the data of the similar bubbles with the original initial conditions (I, III, V, VII, and VIII). We see that when nanobubbles form, they indeed grow towards the same equilibrium size which they also achieve when starting with a finite size bubble. The reason that for these initial conditions in some cases no bubbles form is that the gas concentration $\phi$ in the liquid is not large enough to overcome the nucleation barrier.

\subsection{Continuum description}

In this section, we will use continuum fluid mechanics to explain and predict the behavior of nanobubble clusters. In particular, we will address their stability and calculate their equilibrium size. We will however first address the subject of homogeneous nucleation, which is relevant for the simulations where there was no pre-existing bubble in the initial conditions.

\subsubsection{Nucleation theory}

In the case where there is no pre-existing bubble in the initial conditions (Fig. 3.2b), we are dealing with homogeneous nucleation, since there are no seeds (such as contamination) available to start heterogeneous nucleation [26]. In the case of homogeneous nucleation, the change in free energy 


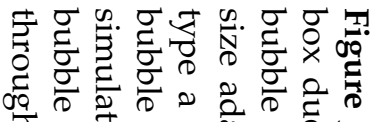

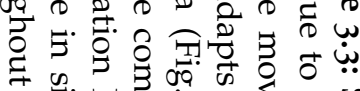

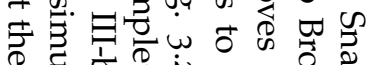

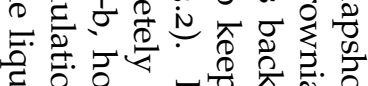

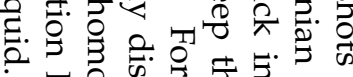

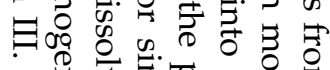

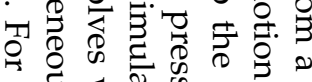

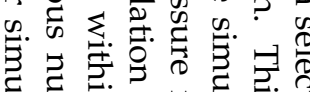

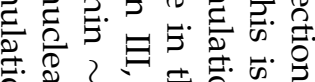

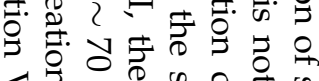

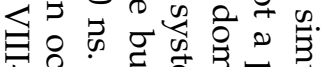

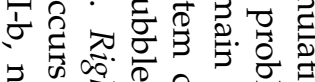

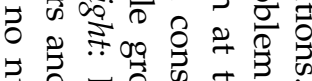

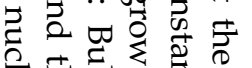

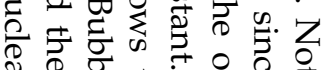

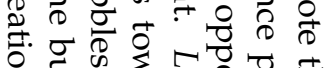

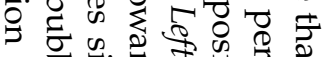

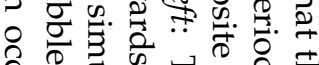

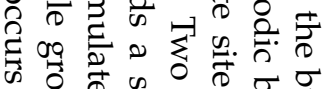

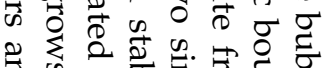
के क्षे

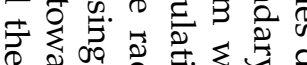

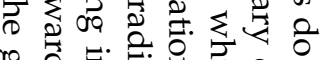

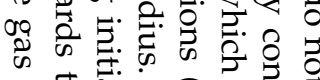

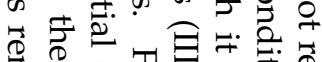

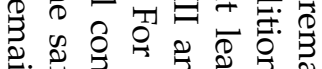
政: S.

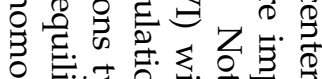
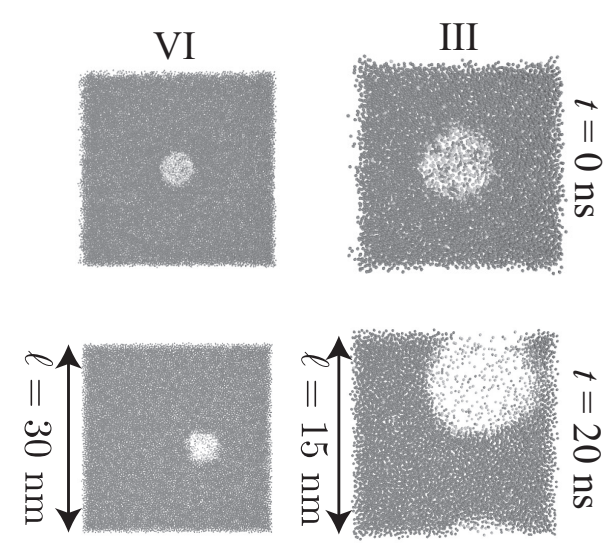
每

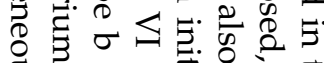

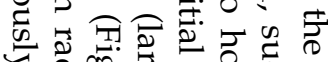

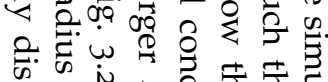

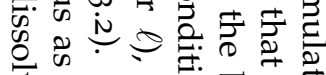

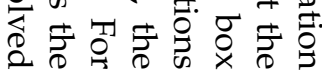
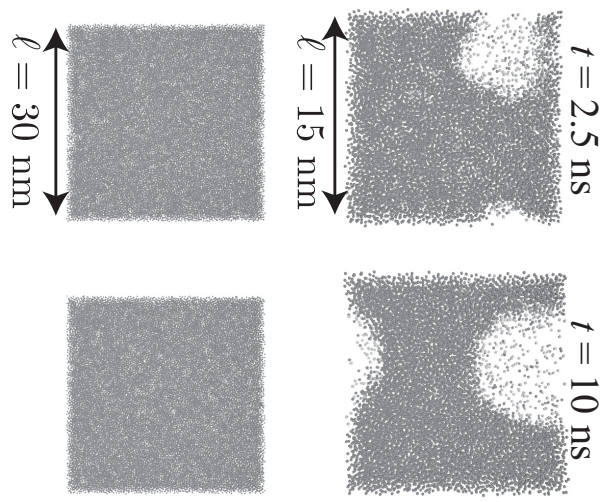


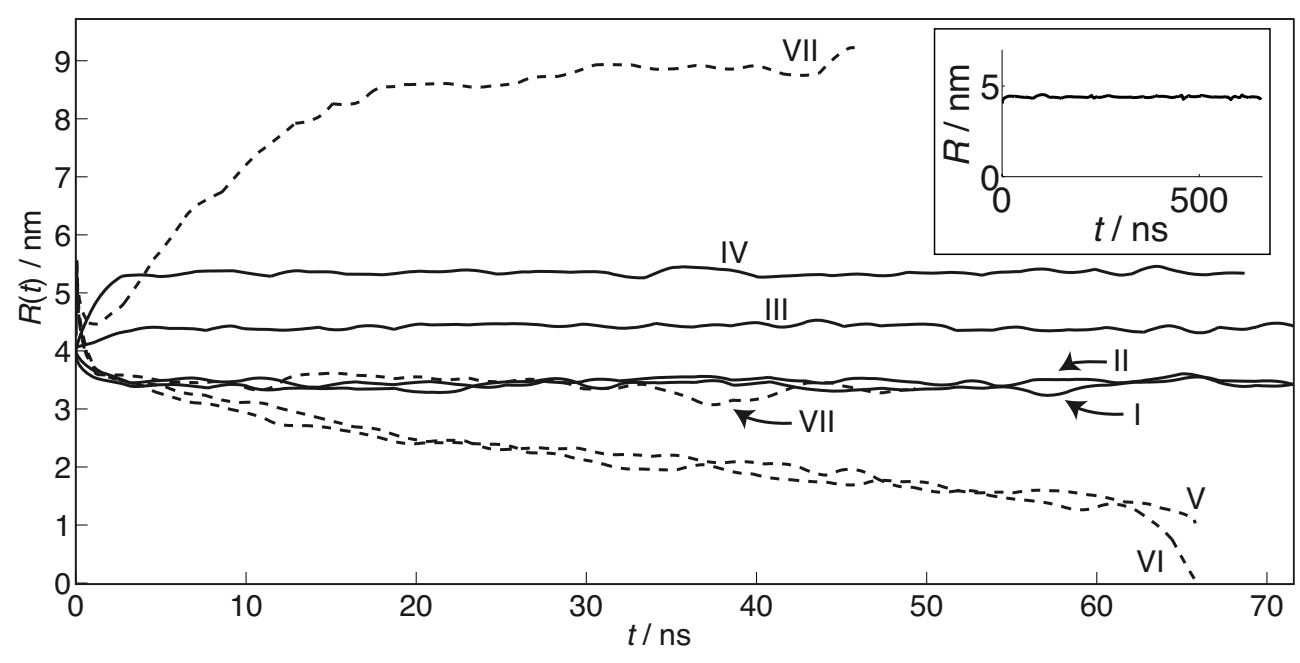

Figure 3.4: Results from the simulations with initial condition type a (Fig. 3.2). All bubbles in the small box $(\ell=15 \mathrm{~nm})$ are stable (I-IV), whereas the simulations in the large box $(\ell=30 \mathrm{~nm})$ and with little gas initially dissolved in the liquid $(\mathrm{V}, \mathrm{VI})$ are unstable. When a sufficient amount of gas is initially dissolved in the liquid (VII, VIII) the bubbles are stable even in the large box. The inset shows the extended simulation of configuration III, to verify that the bubble is indeed stable at long timescales.

of the system when a bubble of radius $R$ forms is:

$$
\Delta G=\frac{4}{3} \pi R^{3} G_{v}+4 \pi R^{2} \gamma
$$

Here, $\Delta G$ is the energy gain or loss for the system to form a bubble of radius $R . G_{v}$ is the (volumetric) energy associated with a unit volume of gas and is a negative number, hence it promotes nucleation. The liquid-vapour surface tension $\gamma$ is always positive and therefore acts agains nucleation. For small $R$, the surface energy term usually dominates, but when the bubble reaches a critical radius $R^{*}=-2 \gamma / G_{v}$ then $\mathrm{d} G /\left.\mathrm{d} R\right|_{R>R^{*}}<0$, meaning that the bubble will grow. In our case, we start out with a bubble of zero radius, but due to thermal fluctuations small bubbles appear randomly throughout the system. By increasing the gas concentration in the liquid, the magnitude of $G_{v}$ increases as it becomes more and more favourable for the system to have gas atoms in the gas phase than in the dissolved state. This decreases the value of $R^{*}$, until it is small enough that the spontaneously forming tiny 


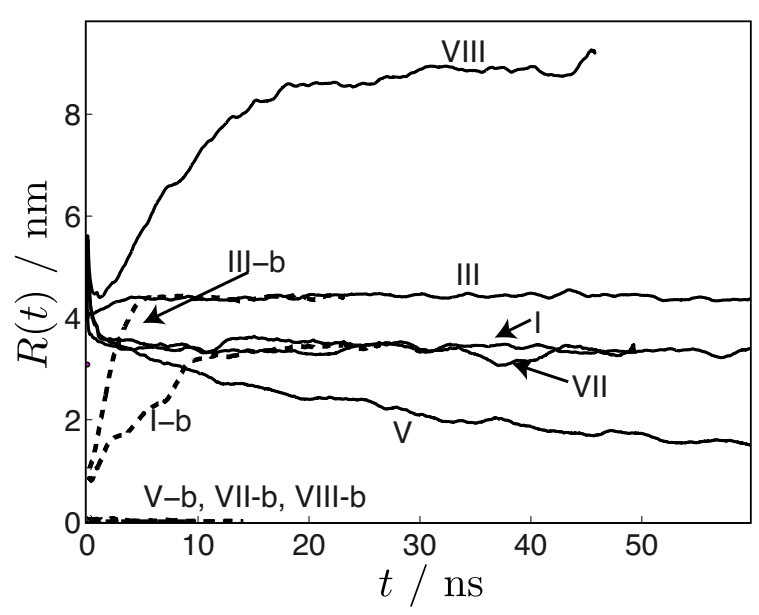

Figure 3.5: Bubble radius evolution as a function of time comparing similar systems with different initial conditions. When bubbles nucleate, they grow towards the same equilibrium size independent of initial conditions. Although a stable bubble exists for configurations VII and VIII, the bubbles in configurations VII-b and VIII-b do not reach this state as the bubbles do not nucleate. Naturally, nucleation also does not occur for the configuration where no stable bubble can exist (V, V-b).

bubbles are already large enough $\left(R>R^{*}\right)$ to overcome the surface energy penalty and grow. The growth stops when equilibrium between the gas and liquid phase is achieved [Eq. (3.5)].

\subsubsection{Growth dynamics}

The stage between nucleation of a gas bubble and it reaching its final size is governed by diffusive bubble growth. The relation between $R$ and $t$ for diffusive bubble growth is well known, namely a square root power law [27, 28],

$$
R(t) \sim(D t)^{\frac{1}{2}},
$$

where $D$ is the diffusion constant of the gas in the liquid. In Fig. 3.6 we show the growth of bubble VIII on a log-log scale. The curve is shifted in time such that $R(t=0)=0$. A power law behaviour with exponent 0.56 is observed. This is very close to the expected exponent of $\frac{1}{2}$, suggesting that the growth of the gas bubbles in the simulation is indeed limited by diffusion. The power law of Eq. (3.4) is derived assuming that the magnitude 


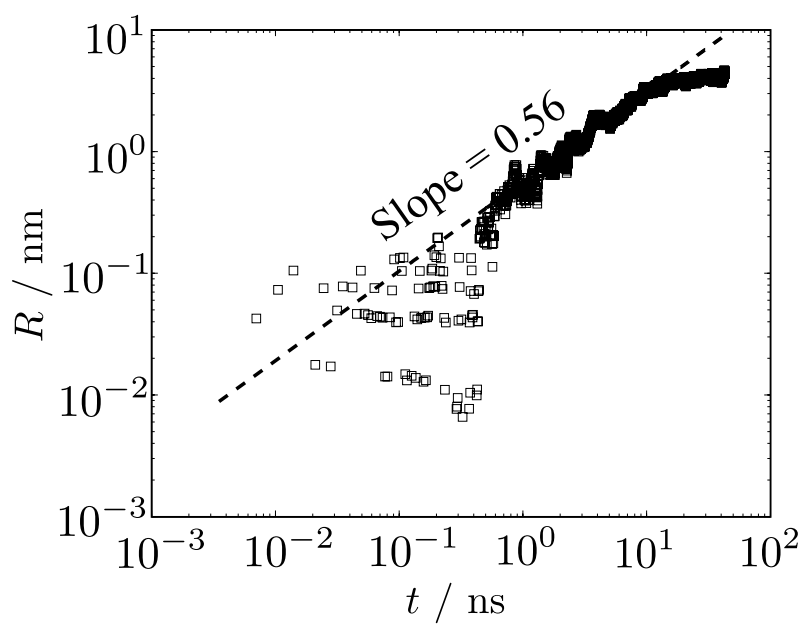

Figure 3.6: Bubble radius as a function of time during growth. The data shown represents bubble VIII, as it grows towards its equilibrium size and has been shifted such that $R(t=0)=0$. At intermediate times an exponent of 0.56 is observed, consistent with diffusive bubble growth. In the final stage the availability of gas is too low to sustain the growth rate, and the bubble settles at its equilibrium radius.

of the Laplace pressure is small compared to atmospheric pressure. This condition is not fulfilled in particular for the tiny bubbles in the beginning, which presumably accounts for the observed deviation from Eq. (3.4). Also, the final stage of bubble growth $(t \gtrsim 10 \mathrm{~ns}$ ) exhibits a different behaviour with time, which is caused by the closed nature of the simulation system and thus the limited amount of gas available. In this stage the available gas in the system is depleted, and the bubble assumes its (final) equilibrium radius.

\subsubsection{Equilibrium radius}

Finally, the bubble reaches its equilibrium radius. When a bubble with radius $R$ exists inside an infinite body of liquid, the concentration of dissolved gas just outside the bubble $(r=R)$ is given by Henry's law:

$$
\frac{p}{\phi}=k_{H} \text {. }
$$

Here, $p$ is the partial pressure of a specific gas in the gas phase, $\phi$ the gas concentration of that specific gas inside the liquid, and $k_{H}$ is Henry's con- 
stant. In this work we will use the (dimensionless) mole fraction of the gas and liquid as the concentration $\phi$. The total pressure in a (2D) bubble is given by:

$$
p_{b}=p_{l}+\frac{\gamma}{R},
$$

where $p_{l}$ is the pressure in the surrounding liquid, which is usually negligible in the case of nanobubbles. From this relation, we can see that surface tension is a strong driving force for dissolution, especially as the bubble radius $R$ becomes small.

$k_{H}$ is Henry's constant and depends on the type of liquid and the type of gas involved. $k_{H}$ is temperature-dependent, but in this work we will consider a system with a fixed temperature. In separate measurements (in a system consisting of a liquid phase in equilibrium with a gas phase at $p=1 \mathrm{~atm})$ we found that $k_{H} \sim 10^{9} \mathrm{~Pa}$ for the Lennard-Jones fluids considered in this work.

A gas concentration gradient induces a diffusive mass flux $J$ according to Fick's law:

$$
J=-D \vec{\nabla} \phi .
$$

In the case of a cluster of bubbles, there is a limited amount of liquid present between neighbouring bubbles. Since the amount is limited (and small if the bubbles are sufficiently close to each other) the concentration is noticeably affected by the gas flow out of the bubbles. Eventually, as more and more gas enters this space, the gas concentration at the mid-point between these bubbles will reach the gas concentration at $R$ as prescribed by Henry's law [Eq. (3.5)]. When this has happened, there is no concentration gradient any more (the concentration is equal everywhere) and no diffusive gas flow exists: The bubbles are stable.

Using Henry's law we can predict the equilibrium size of these nanobubbles. For this, we have to express the concentration of the gas in the liquid as a function of the bubble radius $R$. First, we know that the amount of gas in the liquid is simply given by:

$$
n_{g}^{l}(R)=N_{g}-n_{g}^{b}(R),
$$

where $n_{g}^{l}$ is the number of gas molecules dissolved in the liquid, $N_{g}$ the total number of gas atoms in the system, and $n_{g}^{b}$ the amount of gas molecules inside the bubble. The amount of gas inside the bubble can be related to the 
size of the bubble, using the Laplace pressure and the ideal gas law:

$$
n_{g}^{b}(R)=\frac{2 \pi \gamma R d}{k_{B} T}
$$

Here, $k_{B}$ is Boltzmann's constant, $T$ the temperature of the system. To obtain this relation, we assumed that the Laplace pressure difference is much greater than the ambient pressure, which is a valid assumption for nanobubbles exposed to atmospheric ambient pressure. Henry's law [Eq. (3.5)] dictates the equilibrium condition, so by combining Eqs. (3.8) and (3.9) we can now solve for the equilibrium bubble radius $R_{e q}$ :

$$
R_{e q}=\frac{k_{B} T N_{g}}{4 \pi \gamma d}\left(1 \pm \sqrt{1-4 \frac{\pi \gamma^{2} d N_{l}}{k_{B} T k_{H} N_{g}^{2}}}\right) .
$$

Since the equation is quadratic we get two solutions, of which we cannot say a priori which one is valid, because both are positive and finite.

\subsubsection{Comparison with simulations}

We can now compare the simulation results to the model [Eq. (3.10)].

From Eq. (3.10) it is clear that for a physical solution to exist the number of gas atoms $N_{g}$ must be larger than:

$$
N_{g}^{*}:=\sqrt{\frac{4 \pi \gamma^{2} d N_{l}}{k_{B} T k_{H}}} .
$$

Hence, $N_{g}^{*}$ is a critical amount of gas atoms: When fewer gas atoms are present in the system the bubble will completely dissolve.

In Fig. 3.7 this critical gas content as a function of $N_{l}$, which is proportional to the system size is shown. In the figure the two system sizes considered in this study are annotated. From the figure it is clearly that for larger systems (hence, larger nanobubble seperations and higher $N_{l}$ ) the required amount of gas increases. Intuitively, one would expect that the ratio $N_{g}^{*} / N_{l}$ is constant, but from Eq. (3.11) and Fig. 3.7 we can see that this is not the case. Instead, the minimum number of gas molecules scales with the square root of the amount of liquid present, meaning that it scales linearly with the interbubble distance $\ell$.

The predicted equilibrium radius [Eq. (3.10)] as a function of gas content for the small systems $(\ell=15 \mathrm{~nm})$ and the large systems $(\ell=30 \mathrm{~nm})$ 


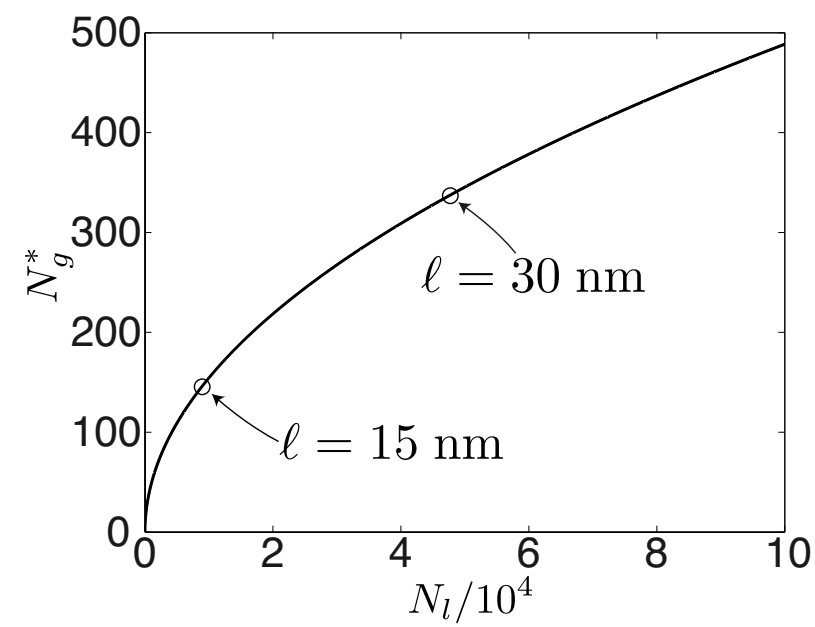

Figure 3.7: Critical number of gas atoms $N_{g}^{*}$ against $N_{l}$. The critical number of gas atoms is the amount of gas atoms required to prevent full dissolution of a nanobubble. As the system is larger (hence larger separation distance between nanobubbles) more gas is required to sustain a stable nanobubble.

are shown in Fig. 3.8(a). The measured equilibrium radii are also depicted, and show excellent agreement with the model. For configurations $\mathrm{V}$ and VI (large systems), the model correctly predicts that there is no stable bubble size, hence they fully dissolve. For configurations VII-b and VIII-b the model predicts that an equilibrium radius does exist, however in these cases there are no bubbles since the nucleation barrier is too high, which is not accounted for in the model. By nondimensionalizing the equilibrium radius $R_{e q}$ with $\ell$, and rescaling $N_{g}$ with $N_{g}^{*}$ we can collapse the data on a single curve, independent of system size, see Fig. 3.8(b).

For $N_{g} \gg N_{g}^{*}$, Eq. (3.10) can be expanded as:

$$
\begin{aligned}
R_{e q}= & \frac{k_{B} T N_{g}}{4 \pi \gamma d} \times\left[1 \pm \sqrt{1-\left(\frac{N_{g}^{*}}{N_{g}}\right)^{2}}\right]= \\
& \frac{k_{B} T N_{g}}{4 \pi \gamma d} \times\left\{\begin{array}{c}
2+\mathcal{O}\left(\left[\frac{N_{g}^{*}}{N_{g}}\right]^{2}\right) \\
\frac{1}{2}\left(\frac{N_{g}^{*}}{N_{g}}\right)^{2}+\mathcal{O}\left(\left[\frac{N_{g}^{*}}{N_{g}}\right]^{4}\right) .
\end{array}\right.
\end{aligned}
$$

These asymptotic solutions are plotted in Fig. $3.8 \mathrm{~b}$ as the black dashed lines. 

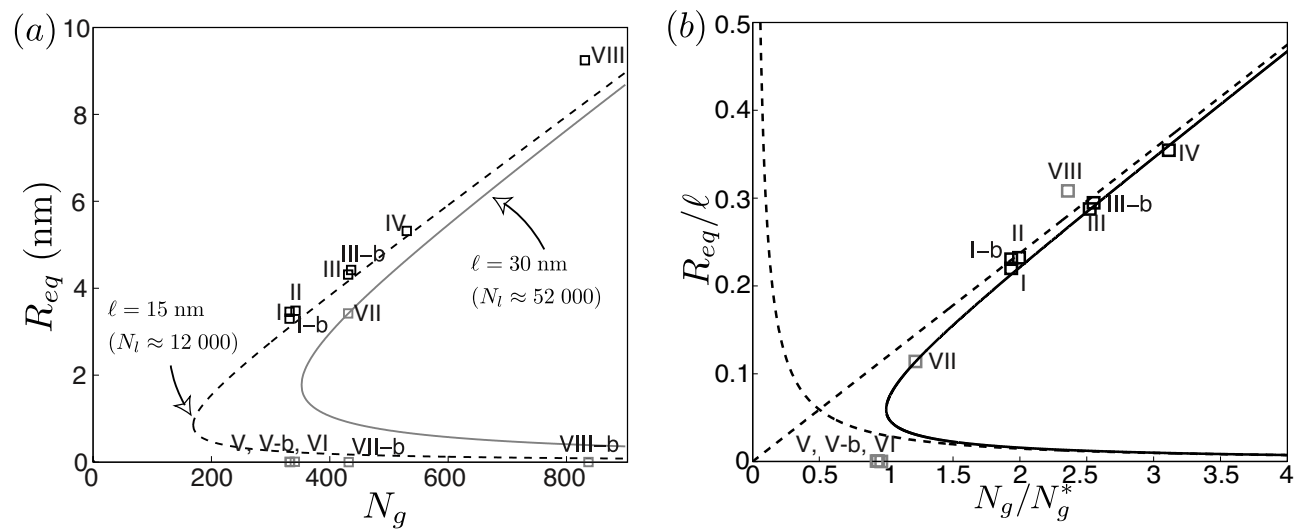

Figure 3.8: (a) Equilibrium radii $R_{e q}$ as measured in the simulations (symbols) and predicted by the model (lines) as a function of $N_{g}$. The black data correspond to the small system $(\ell=15 \mathrm{~nm})$, whereas the gray data correspond to the large system $(\ell=30 \mathrm{~nm})$. The model nicely predicts the equilibrium size in both systems, but of course does not account for the configurations where no bubble nucleation occurs (V-b, VII-b, and VIII-b). To obtain the theoretical curves, a value of $k_{H}=$ $1.5 \cdot 10^{9} \mathrm{~Pa}$ was used as a fit parameter. Independent measurements have shown $k_{H} \sim 10^{9} \mathrm{~Pa}$. (b) Collapse of the nondimensionalized equilibrium radii. All data points nicely follow the master curve. The simulations where no nucleation occurs are not included in this plot, to emphasize the good prediction of both $R_{e q}$ and $N_{g}^{*}$ by the model. The dashed lines indicate the expansion when $N_{g} \gg N_{g}^{*}$, Eq. (3.12).

The first solution of Eq. (3.12) represents the limit where all gas atoms are contained within the bubble. This can also be seen by comparing this result with Eq. (3.9). It is apparent from Fig. 3.8 that almost all bubbles are very close to this limit i.e. there is virtually no gas dissolved inside the liquids in the simulations.

\subsection{Nanobubble cluster stability}

Finally, we will discuss the stability of the entire cluster of nanobubbles. Although it is now clear that bubbles that are surrounded by mirror images can indeed be in equilibrium, this does not mean that the entire cluster is stable. There are two obvious threats to this stability: (i) the Brownian motion of bubbles can cause neighboring bubbles to collide, leading to coalescence and coarsening of bubbles within the cluster and (ii) when neighboring bub- 
bles are not exactly equally sized, smaller bubbles would drain into larger bubbles via diffusion (similar to Ostwald ripening).

First, we address the possibility of bubbles colliding. Since the distance between neighbouring bubbles is at best ten times as large as the bubble radius, Brownian motion of nanobubbles can indeed lead to collisions (see Fig. 3.3, where the nanobubbles are shown to move around). An obvious way to prevent this from happening is to make sure that the bubbles repel each other. This repulsion could be electrostatic (e.g. by using an ionic surfactant, or by the intrinsic negative charge of air bubbles in water [2931]), which is sufficiently long-ranged. Adding salt to the solution, which leads to screening of the electrostatic fields around the nanobubbles, would then reduce the stability of bulk nanobubble clusters which has indeed been observed in experiments [5].

The second issue (Ostwald ripening) is harder to prevent, and we expect that in time coarsening would indeed occur. Of course, the larger bubbles that are formed in this way are still able to provide some shielding, but the polydispersity of bubble sizes will make a theoretical analysis difficult. Therefore, at this point, we do not have an explanation for the measured stability by Ohgaki et al. [2] where Ostwald ripening apparently has been suppressed.

\subsection{Conclusions}

In conclusion, we have shown that bulk nanobubble clusters can indeed be stable under specific conditions. First, the individual bubbles are surrounded by similar nanobubbles. The distance between these bubbles must be small enough, such that the bubbles can succeed in saturating the liquid between the bubbles with gas, before the bubbles are completely drained. When a cluster of stable bubbles exist, and the system is closed (so no gas can escape) the bubbles will in principle live forever, as long as bubbles cannot merge and Ostwald ripening is somehow prevented. The merging of bubbles can be prevented by ionic surfactants. Ostwald ripening is harder to prevent in theory, but experimental results showing that nanobubble cluster can indeed be stable for longer times indicate that there exists a mechanism that can prevent Ostwald ripening from occuring $[2,5,6]$. 


\section{References}

[1] P. Epstein and M. Plesset, On the stability of gas bubbles in liquid-gas solutions, J. Chem. Phys. 18, 1505 (1950).

[2] K. Ohgaki, N. Q. Khanh, Y. Joden, A. Tsuji, and TakaharuNakagawa, Physicochemical approach to nanobubble solutions, Chem. Eng. Sci. 65, 1296 (2010).

[3] A. Häbich, W. Ducker, D. E. Dunstan, and X. Zhang, Do Stable Nanobubbles Exist in Mixtures of Organic Solvents and Water?, The Journal of Physical Chemistry B 114, 6962 (2010).

[4] T. Uchida, S. Oshita, M. Ohmori, T. Tsuno, K. Soejima, S. Shinozaki, Y. Take, and K. Mitsuda, Transmission electron microscopic observations of nanobubbles and their capture of impurities in wastewater, Nanoscale Research Letters 6, 295 (2011).

[5] F. Jin, X. Ye, and C. Wu, Observation of Kinetic and Structural Scalings during Slow Coalescence of Nanobubbles in an Aqueous Solution, J. Phys. Chem. B 111, 13143 (2007).

[6] F. Jin, J. Ye, L. Hong, H. Lam, and C. Wu, Slow Relaxation Mode in Mixtures of Water and Organic Molecules: Supramolecular Structures or Nanobubbles?, J. Phys. Chem. B 111, 2255 (2007).

[7] M. A. Hampton and A. V. Nguyen, Nanobubbles and the nanobubble bridging capillary force, Adv. Colloid Interface Sci. 154, 30 (2010).

[8] V. S. J. Craig, Very small bubbles at surfaces-the nanobubble puzzle, Soft Matter 7, 40 (2011).

[9] J. R. T. Seddon and D. Lohse, Nanobubbles and micropancakes: gaseous domains on immersed substrates, J. Phys.: Condens. Matter 23, 133001 (2011).

[10] J. Parker, P. Claesson, and P. Attard, Bubbles, cavities, and the long-ranged attraction between hydrophobic surfaces, J. Phys. Chem. 98, 8468 (1994).

[11] S. Lou, Z. Ouyang, Y. Zhang, X. Li, J. Hu, M. Li, and F. Yang, Nanobubbles on solid surface imaged by atomic force microscopy, J. Vac. Sci. Technol. B 18, 2573 (2000). 
[12] J. W. G. Tyrrell and P. Attard, Images of Nanobubbles on Hydrophobic Surfaces and Their Interactions, Phys. Rev. Lett. 87, 176104 (2001).

[13] M. Holmberg, A. Kühle, , K. A. Mørch, and A. Boisen, Nanobubble Trouble on Gold Surfaces, Langmuir 19, 10510 (2003).

[14] R. Steitz, T. Gutberlet, T. Hauss, B. Kløsgen, R. Krastev, S. Schemmel, A. C. Simonsen, and G. H. Findenegg, Nanobubbles and Their Precursor Layer at the Interface of Water Against a Hydrophobic Substrate, Langmuir 19, 2409 (2003).

[15] A. C. Simonsen, P. L. Hansen, and B. Klösgen, Nanobubbles give evidence of incomplete wetting at a hydrophobic interface, J. Colloid Interface Sci. 273, 291 (2004).

[16] X. H. Zhang, X. D. Zhang, S. T. Lou, Z. X. Zhang, J. L. Sun, and J. Hu, Degassing and Temperature Effects on the Formation of Nanobubbles at the Mica/Water Interface, Langmuir 20, 3813 (2004).

[17] B. M. Borkent, S. M. Dammer, H. Schönherr, G. J. Vancso, and D. Lohse, Superstability of Surface Nanobubbles, Phys. Rev. Lett. 98, 204502 (2007).

[18] S. Yang, S. M. Dammer, N. Bremond, H. J. W. Zandvliet, E. S. Kooij, and D. Lohse, Characterization of Nanobubbles on Hydrophobic Surfaces in Water, Langmuir 23, 7072 (2007).

[19] S. Yang, E. S. Kooij, B. Poelsema, D. Lohse, and H. J. W. Zandvliet, Correlation between geometry and nanobubble distribution on HOPG surface, Europhys. Lett. 81, 64006 (2008).

[20] W. A. Ducker, Contact Angle and Stability of Interfacial Nanobubbles, Langmuir 25, 8907 (2009).

[21] M. P. Brenner and D. Lohse, Dynamic Equilibrium Mechanism for Surface Nanobubble Stabilization, Phys. Rev. Lett. 101, 214505 (2008).

[22] J. R. T. Seddon, H. J. W. Zandvliet, and D. Lohse, Knudsen Gas Provides Nanobubble Stability, Phys. Rev. Lett. 107, 116101 (2011).

[23] T. Yamamoto and S. Ohnishi, Molecular dynamics study on helium nanobubbles in water, Phys. Chem. Chem. Phys. 13, 16142 (2011). 
[24] W. Chun-Lei, L. Zhao-Xia, L. Jing-Yuan, X. Peng, H. Jun, and F. HaiPing, High density gas state at water/graphite interface studied by molecular dynamics simulation, Chinese Physics B 17, 2646 (2008).

[25] G. Bussi, D. Donadio, and M. Parrinello, Canonical sampling through velocity rescaling, J. Chem. Phys. 126, 014101 (2007).

[26] J. Schmelzer, Nucleation theory and applications (Wiley-VCH, New York, 2005).

[27] C. E. Brennen, Fundamentals of multiphase flow (Cambridge University Press, New York, 2005).

[28] G. Y. Gor and A. E. Kuchma, Dynamics of gas bubble growth in a supersaturated solution with Sievert's solubility law, J. Chem. Phys. 131, 034507 (2009).

[29] A. Graciaa, G. Morel, P. Saulner, J. Lachaise, and R. Schechter, The Zetapotential of gas-bubbles, J. Colloid Interface Sci. 172, 131 (1995).

[30] M. Takahashi, $\zeta$ Potential of Microbubbles in Aqueous Solutions: Electrical Properties of the Gas-Water Interface, J. Phys. Chem. B 109, 21858 (2005).

[31] P. Creux, J. Lachaise, A. Graciaa, and J. K. Beattie, Specific Cation Effects at the Hydroxide-Charged Air/Water Interface, J. Phys. Chem. C 111, 3753 (2007). 


\section{4 \\ Why surface nanobubbles live for hours*}

We present a theoretical model for the experimentally found but counter-intuitive exceptionally long lifetime of surface nanobubbles. We can explain why, under normal experimental conditions, surface nanobubbles are stable for many hours or even up to days rather than the expected microseconds. The limited gas diffusion through the water in the far field, the cooperative effect of nanobubble clusters, and the pinned contact line of the nanobubbles lead to the slow dissolution rate.

\subsection{Introduction}

Since their first prediction and discovery almost 20 years ago [1], intense research on surface nanobubbles has raised many questions about this intriguing and important phenomenon which has great potential for various applications [2-5]. Surface nanobubbles have now been widely reported on various surfaces in contact with water employing various detection mechanisms like atomic force microscopy (AFM) and most recently also through direct optical visualization $[6,7]$. With all these different methods they are found to behave differently than regular macroscopic bubbles. Surface nanobubbles behave peculiarly in several ways: their contact angle is always much

*Published as: J.H. Weijs and D. Lohse, "Why surface nanobubbles live for hours", Phys. Rev. Lett. 110, 054501 (2013). 
lower than expected from Young's law [8, 9]; they are stable against violent decompression [10]; and in particular they are stable for much longer than expected: For such small bubbles one would expect a lifetime of order $\mu \mathrm{s}$, due to the high Laplace pressure inside the bubbles that drives the gas into the liquid. On this last question many explanations were proposed, ranging from contamination that shields or limits the diffusive outflux of gas [11] to a dynamic equilibrium situation where lost gas is replenished [12, 13]. However, both theories are refuted by experimental evidence: the addition of surfactants does not influence the behaviour of nanobubbles [14], and the circulatory gas flow required for the dynamic equilibrium theory is not measured in all experiments so it cannot be the stabilization mechanism [7, 13]. In addition, a large problem with the dynamic equilibrium theory is that it requires some form of driving to satisfy the second law of thermodynamics, and its origin is unclear. In molecular dynamics, some local inflow near the contact line was indeed observed, but its strength was too weak to explain the stability of surface nanobubbles, see chapter 2.

A different approach is therefore required, and in this chapter we provide an alternative explanation for the long lifetimes of surface nanobubbles. The theory relies only on classical, well-known, and proven continuum concepts such as diffusion and Henry's law. Furthermore, the theory only uses confirmed nanobubble properties, namely the pinned contact line $[15,16]$ of nanobubbles and the fact that nanobubbles exist in relatively high coverage fractions at the liquid-solid interface [17, 18]. No fitting or uncontrolled assumptions are required to obtain lifetimes that are consistent with experimental findings. The pinned contact line of surface nanobubbles has, until now, received little attention but it turns out to be crucial for long nanobubble lifetimes. The origin of the pinned contact lines is beyond the scope of this work and here we will only address the lifetime question of surface nanobubbles, which has been puzzling the community ever since the first discovery of surface nanobubbles [1].

This chapter is organized as follows. First, the theory is explained and the relevant equations are derived. Next, we solve the equations numerically and analytically. We vary several parameters to demonstrate the robustness of the long lifetimes of surface nanobubbles in varying experimental conditions. In addition, we apply the theory to the case of electrolytically generated nanobubbles and find that here also it is consistent with experimental results. We conclude with predictions from the theory that can straightforwardly be tested in experiments. 


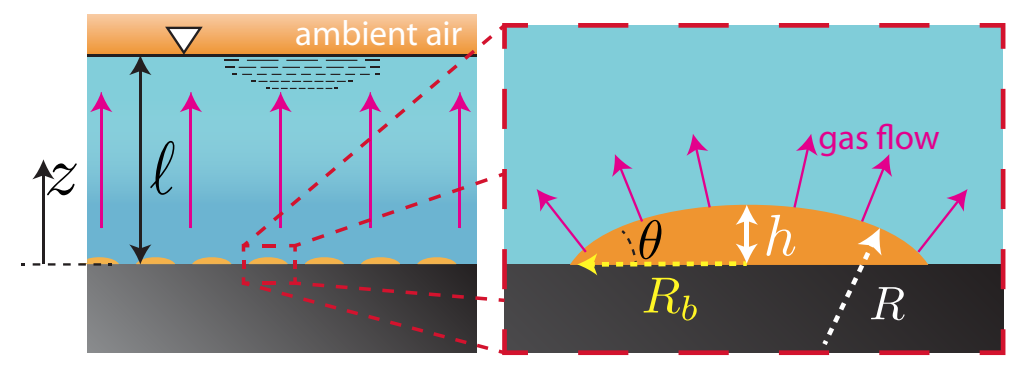

Figure 4.1: Sketch of a liquid layer of thickness $\ell$ in contact with a solid (left). The top of the liquid is exposed to atmospheric conditions. At the solid-liquid interface nanobubbles are present with typical internal contact angle $\theta$, height $h$, and radius of curvature $R$. They are not drawn to scale. The arrows indicate the gas flow direction. On the right a further enlargement of one nanobubble is shown.

\subsection{Theory}

We consider an infinitely large plate in contact with a liquid layer with thickness $\ell$ (Fig. 4.1). The liquid layer is in contact with the atmosphere at $z=\ell$ and the solid-liquid interface is located at $z=0$. The solid-liquid interface is covered with nanobubbles with a number density (per area) of $\rho$, hence the (average) spacing between neighbouring nanobubbles is $\left\langle d_{b u b}\right\rangle \approx \frac{1}{\sqrt{\rho}}$. In experimental studies nanobubbles are always recovered in high coverage densities [17-20]. Assuming quasi-steady diffusion, any variation of the dissolved gas concentration $\phi$ in the horizontal $(x, y)$ direction due to nonuniform gas outflux decays as $\exp \left(-\pi z / d_{b u b}\right)$. Hence we can assume that for $\ell>5\left\langle d_{b u b}\right\rangle$ the diffusion of gas through the liquid layer is governed by the one-dimensional diffusion equation:

$$
\frac{\partial \phi}{\partial t}=D \frac{\partial^{2} \phi}{\partial z^{2}}, z \in[0, \ell],
$$

with $D$ the diffusion constant of the gas in the ambient liquid and $\phi$ the (number) concentration of gas in the liquid. The characteristic time scale for diffusion through the water layer is $\ell^{2} / D \sim 10^{5} \mathrm{~s}$, which is similar to the lifetimes obtained in experiments. The vast difference ( $\mu$ s vs. days) compared to previous estimates originates in using $\ell$ as the relevant length scale instead of the bubble radius $R$, i.e. we use the far-field length scale. Using $R$ as the length scale is justified for a (free) bubble in an infinite medium. However, in the case of nanobubbles it is important to realize that gas has 
not left the system until it is released into the atmosphere and hence $\ell$ is the relevant length scale as this is the distance the gas has to travel through the liquid from the bubble toward the atmosphere. This is also apparent in a thought experiment where we do not allow gas to leave the liquid into the atmosphere, for example by putting the liquid drop in a closed container. Because the liquid is supersaturated with some gas that has left the bubble, and which cannot escape, an equilibrium is reached (described by Henry's law discussed later in this work) and the bubbles do not dissolve as is the case with the bulk nanobubbles discussed in chapter 3. When opening the container this excess dissolved gas can be released into the ambient air, allowing the bubble to lose gas into the liquid. This "traffic jam effect" plays a crucial role in the long nanobubble lifetimes.

We will now fully describe the boundary conditions for the above differential equation (4.1). They are given by Henry's law, which relates the gas concentration in the liquid to the gas pressure outside the liquid near the interfaces:

$$
\phi(z=0, t)=\frac{p_{b u b}(R(N(t)))}{k_{H}} \text { and } \phi(z=\ell, t)=\frac{p_{a t m}}{k_{H}} .
$$

Here, $k_{H}$ is Henry's constant, $p_{b u b}$ the pressure inside the nanobubbles and $p_{a t m}$ the atmospheric pressure. $R(t)$ is the radius of curvature of the nanobubbles, which depends on time because the bubbles get flatter as they drain. In the case of a pinned contact line, the radius of curvature is related to the (internal) contact angle $\theta(t)$ by $R(t)=\frac{R_{b}}{\sin \theta(t)}$, where $R_{b}$ is the base radius (cf. Fig. 4.1) which is constant due to the pinned contact line. The (relative) pressure inside the bubbles $p_{b u b}(t)$ is the Laplace pressure

$$
p_{b u b}(t)=p_{a t m}+\frac{2 \gamma}{R}=p_{a t m}+\frac{2 \gamma}{R_{b}} \sin \theta(t),
$$

where $\gamma$ is the liquid-vapour surface tension. For $\theta<90^{\circ}$, which is the case for surface nanobubbles, the internal pressure thus decreases as $\theta$ decreases. This effect provides a negative feedback in the dissolution process, prolonging the lifetime of the nanobubbles. In this work we do not consider the effects of electrostatic effects on the internal pressure of surface nanobubbles, as electrostatic effects act to reduce the internal pressure and are therefore not a driving force but rather a stabilizing force. It is therefore possible that the derived lifetimes in this work (hours, days) are an underestimation of real lifetimes. 
The (single) nanobubble gas content $N(t)$ decreases due to the diffusive flux of gas at $z=0$, which is the location at which gas is transferred from the nanobubbles $\left(z=0^{-}\right)$to the liquid $\left(z=0^{+}\right)$. The diffusive flux is given by Fick's law $J=-D(\partial \phi / \partial z)$, thus

$$
\frac{\mathrm{d} N}{\mathrm{~d} t}=-\frac{J}{\rho}=\left.\frac{D}{\rho} \frac{\partial \phi(z, t)}{\partial z}\right|_{z=0} .
$$

The factor $\rho$ arises to convert the molecular flux per unit area of the substrate to the molecular flux per single surface nanobubble. Eq. (4.4) immediately shows how a low nanobubble coverage $(\rho \approx 0)$ corresponds to small global flux, $J=-\rho d N / d t$.

To evaluate the boundary condition at $z=0$ (Eq. (4.2)), we need to relate the number of atoms inside a nanobubble (Eq. (4.4)) to the geometrical shape of the bubble. To calculate the geometrical properties of a nanobubble containing $N$ atoms of gas, we use the ideal gas law $p(\theta) V(\theta)=N k_{B} T$ using the expression for the volume of a spherical cap $V(\theta)=\frac{\pi}{3}\left(R_{b} / \sin \theta\right)^{3} \cdot(2-$ $\left.3 \cos \theta+\cos ^{3} \theta\right)$. Here, $k_{B}$ is Boltzmann's constant, $T$ the temperature (assumed to be constant at $300 \mathrm{~K}$ ), and $\theta$ the gas-side (internal) contact angle of the nanobubbles. This implicit equation can be solved numerically for $\theta(N)$. We can then calculate the radius of curvature of the nanobubbles $R$, which gives us the internal bubble pressure [Eq. (4.3)]. Using this pressure, the boundary condition at $z=0$ (Eq. (4.2)) can be evaluated, which closes our model. In the next section, we will solve these model equations numerically.

\subsection{Numerical evaluation}

Due to the nontrivial boundary condition at $z=0$ (Eq. (4.2)) we first solve the diffusion eq. (4.1) numerically. The simulations were done for different initial conditions. Because the real initial conditions are unknown, we choose the two extremes between which we expect the real initial conditions. The first type of initial conditions consists of a linear concentration profile, which allows the system to begin transporting gas from the bubbles immediately $(t=0)$,

$$
\phi(z, t=0)=\frac{p_{b u b}\left(R_{0}\right)-p_{a t m}}{k_{H}}\left(1-\frac{z}{\ell}\right)+\frac{p_{a t m}}{k_{H}} .
$$

Here, $R_{0}$ indicates the initial radius of curvature of the nanobubbles. We choose $R_{0}$ such that it is equivalent to an initial contact angle $\theta_{0}=40^{\circ}$ for given base radius $R_{b}$. 

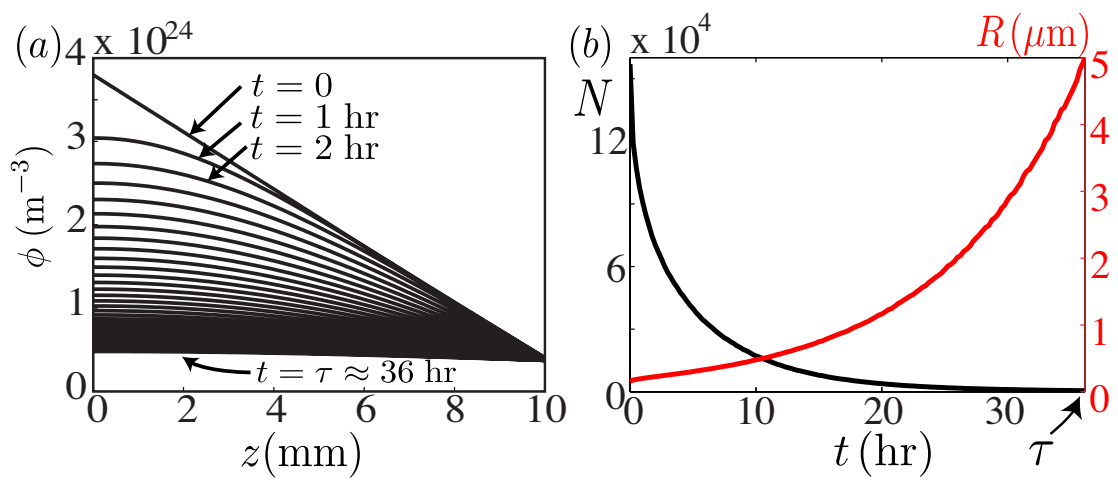

Figure 4.2: Results of the calculations using the initial conditions from Eq. (4.5) and parameters described in the text. (a): Snapshots of the concentration profile at 1 hour intervals. Because the bubbles are pinned, the radius of the curvature increases as the bubble drains, lowering the concentration at the bubble side $(z=0)$. (b): Evolution of the nanobubble gas content (black curve) and the radius of curvature of the liquid-gas interface $R$ (red curve) as a function of time.

The second type of initial conditions assumes that the nanobubble formation procedure (ethanol-water exchange or replacing cold water with warm water [20]) supersaturates and mixes the water such that the concentration is uniform and equal to the concentration near the nanobubbles:

$$
\phi(x, t=0)=\frac{p_{b u b}\left(R_{0}\right)}{k_{H}} .
$$

The real initial concentration profile will most likely be something between (4.5) and (4.6): the ethanol-water exchange uniformly supersaturates the water but it takes the nanobubbles some time to form so some gas already drains into the atmosphere. As we will see, both initial concentration profiles produce long-living nanobubbles.

\subsection{Results}

How long does a nanobubble survive according to this description? Using typical parameters that apply to experiments on surface nanobubbles [8] $\left(\tilde{\rho}=4 \cdot 10^{12} \mathrm{~m}^{-2}, \ell=10^{-2} \mathrm{~m}, \gamma=0.072 \mathrm{~N} / \mathrm{m}, D=10^{-9} \mathrm{~m}^{2} / \mathrm{s}, k_{H}=2.6\right.$. $10^{-19} \mathrm{~Pa} \cdot \mathrm{m}$, and $T=300 \mathrm{~K}$ ) and the initial condition (4.5) we obtain the 
results shown in Fig. 4.2. Fig. 4.2(a) shows hourly snapshots of the concentration profile $\phi(z)$. From these curves it is apparent that the transport of gas away from the bubble is limited by the diffusion rate of the gas through water far away from the bubble. This leads to an almost flat (zero-slope) concentration profile near the bubble through which the diffusive gas flux is very small. Fig. 4.2(b) (red curve) shows the radius of curvature of the spherical cap $R$ as a function of time. This radius of curvature increases (due to the pinned contact line) which lowers the internal gas pressure [Eq. (4.3)], enhancing the lifetime of the nanobubbles. Finally, Fig. 4.2(b) (black curve) shows the amount of particles contained inside an individual nanobubble through time. Here we see that a typical nanobubble that starts out with over 100,000 atoms and ends up with just over 500 atoms after 36 hours.

As a criterion for bubble dissolution we choose a critical bubble height of $h^{*}=1 \mathrm{~nm}$, after which it may no longer be appropriate to use continuum physics, and also effects such as the disjoining pressure start to dominate the pressure inside the bubble rather than solely the Laplace pressure. From molecular dynamics it is known that continuum models (e.g. Navier-Stokes, diffusion equation, Henry's law) are valid down to the nanometer scale, see also chapters 2 and 3 . Using the same criterion we find that the above bubble is stable for 36 hours, which is 10 orders of magnitude longer than previously thought and in agreement with experimental findings and the expected timescale $\ell^{2} / D$.

\subsection{Analytic solution}

The concentration profiles in Fig. 4.2 suggest that the boundary condition at $z=0$ is of Neumann type, $\partial \phi /\left.\partial z\right|_{z=0}=0$. Taking the time derivative of the boundary condition at $z=0$ [Eq. (4.2)] and substituting Eq. (4.4) into the result we obtain

$$
\left.\frac{\partial \phi}{\partial z}\right|_{z=0}=\left.\frac{\rho}{D \frac{d \phi}{d N}} \frac{\partial \phi}{\partial t}\right|_{z=0} .
$$

Filling in representative values for the quantities we find that the concentration gradient at $z=0$ is over 5 orders of magnitude smaller than the typical global concentration gradient $(\approx \hat{\phi} / \ell)$, with $\hat{\phi}=\phi-\phi_{a t m}$. This means that the boundary condition for the gradient at $z=0$ can indeed be considered to be approximately zero. For zero gradient the analytic solution is $\hat{\phi}(z, t) \sim \sum_{n=1}^{\infty} \exp \left(-n^{2} \pi^{2} D t /\left(4 \ell^{2}\right)\right) \cdot \cos (n \pi z /(2 \ell))$. We only take into account the first mode $(n=1)$, which has the smallest time constant and there- 
fore remains after any initial effects due to arbitrary initial conditions have declined. A remarkable feature of this result is that $\hat{\phi}(z, t)$ only depends on $D$ and $\ell$, and is completely independent of $\rho$ and $\gamma$. Of course, $\rho$ must be high enough to be able to consider the system as one-dimensional. Similarly, the fraction $\rho /(D d \phi / d N)$ must be low enough such that the local gradient at $z=0$ [Eq. (4.7)] is small compared to the global gradient.

\subsection{Varying initial conditions}

How does the initial concentration profile affect the lifetime of the nanobubbles? During the ethanol-water exchange procedure that is most commonly used to generate nanobubbles experimentally, ethanol is flushed away with clean water. It is therefore likely that the initial gas concentration profile is uniform in $z$, due to mixing. We redid the same calculations as before, using an uniform initial concentration profile, and the results are plotted in Fig. 4.3. We observe very similar concentration profiles as before, except for small times where the influence of the initial conditions is still felt. As can be observed in Fig. 4.3 (b), it takes 2-3 hours before the bubbles "feel" the influence of the ambient air and start to dissolve. This means that during these first hours, the bubbles barely shrink as the (global) concentration gradient near the bubbles is close to zero.

\subsection{Robustness of the results}

How robust are the nanobubble lifetimes against variations in the experimental system? By changing $\gamma$ and $\ell$ in the numerical calculation, we verify the result from the analytical solution to the diffusion equation for which it holds that the diffusion profile evolution only depends on $D$ and $\ell$.

First, we look at the surface tension $\gamma$. Most often an ethanol-water exchange procedure is applied to form nanobubbles. This method introduces contamination into the system which lowers the surface tension. It is therefore important to understand the influence of $\gamma$ on the nanobubble lifetime. We find that surface tension does not play any role in the dissolution time of nanobubbles. This result remains counter-intuitive as surface tension is the driving force for nanobubble dissolution. Indeed, a higher surface tension increases the Laplace pressure [Eq. (4.3)], thus increasing the driving that leads to dissolution. However, it also increases the gas content inside the 

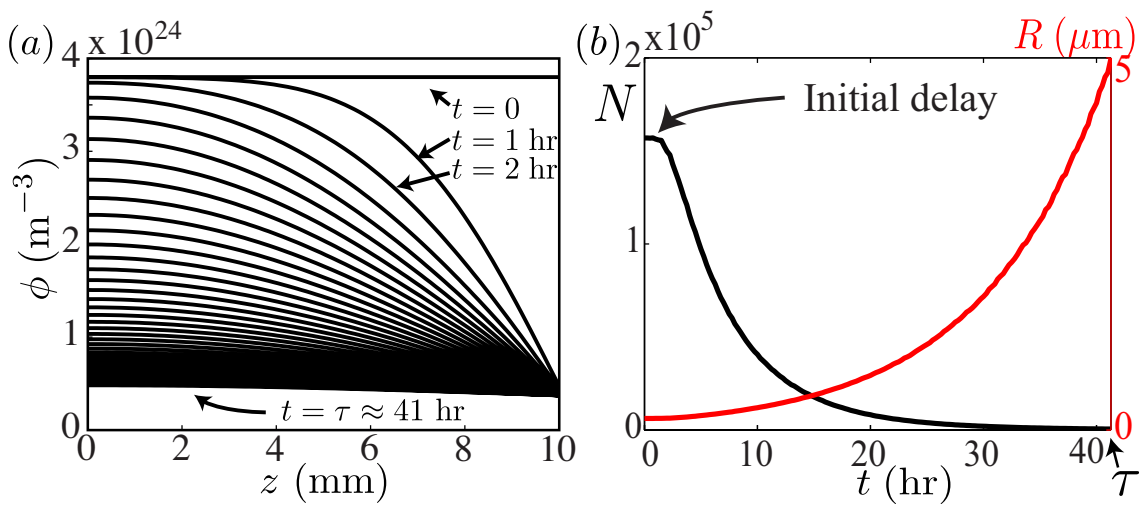

Figure 4.3: Numerical results for the initial conditions (4.6). (a): Hourly snapshots of the concentration profile. Due to the initial conditions, some gas that is initially dissolved in the liquid first has to drain to the atmosphere until a concentration gradient exists near the bubble. As compared to the linear profile case (Fig. 4.2) the bubbles gain an additional few hours of lifetime because of this. (b): Evolution of the nanobubble gas content and the radius of curvature of the surface nanobubbles.

bubbles. This denser reservoir requires a larger flux to drain in the same time. Both effects scale linearly with $\gamma$; hence, they cancel.

In previous studies, the liquid height $\ell$ has never been considered as a parameter that affects nanobubble lifetimes. Based on the analytical solution presented before we expect that $\tau \sim \ell^{2} / D$, which is indeed exactly recovered from the numerical calculations. This indicates that the liquid cell size (and geometry) is very important to the lifetime of nanobubbles: the amount of liquid the gas has to travel through determines the timescale of the nanobubble lifetime. $\ell$ is easy to vary, we suggest performing corresponding experiments to test our prediction.

\subsection{Electrolysis}

How do nanobubbles behave according to this description when gas is generated at the solid-liquid interface (e.g. by electrolysis [15, 21])? A rough estimate based on the values cited in ref. [15] gives a constant influx of order $\sim 10^{5}$ molecules per bubble per second. For an equilibrium to exist (the bubbles neither grow or shrink) the diffusive flux away from the bubble must 
then be equal to this influx due to electrolysis, hence $\frac{D}{\rho} \hat{\phi}(z=0) / \ell=10^{5} \mathrm{~s}^{-1}$. This corresponds to a nanobubble contact angle of $\theta=49^{\circ}$, and nanobubble content $N=2 \cdot 10^{5}$. Interestingly, this means that the bubble contents are refreshed every two seconds. This only highlights the fact that nanobubbles are not static: without driving (such as electrolysis) they dissolve whereas with driving the gas atoms inside the bubble are replaced every couple of seconds.

\subsection{Conclusion}

We have modelled the gas flow from nanobubbles through the liquid to the atmosphere to study the lifetime of surface nanobubbles. We find that nanobubbles are not stable, but dissolve by diffusion. However, due to their pinned contact lines and because the gas has to diffuse toward the atmosphere, they dissolve on a much longer time scale than free bubbles in an infinite liquid. This last point (diffusion through a relatively thick layer of liquid) explains why previous molecular dynamics results could not recover long nanobubble lifetimes: the system size in molecular dynamics is limited to several tens of nanometers, resulting in lifetimes of order $100 \mathrm{~ns}$, consistent with our findings in chapter 2 . Using the correct length scales $(\ell \sim 1-10 \mathrm{~mm})$, we find that surface nanobubbles can easily survive in excess of a day, an increase of 10 orders of magnitudes as compared to previous estimates of $\mu \mathrm{s}$. The results from the experiment where electrolysis is used corroborate the results in this Letter: a nonzero current is measured meaning that gas is continuously formed at the substrate. We find that the gas flux induced by this current leads to nanobubbles with $\theta=46^{\circ}$, consistent with the experimental results of ref. [15].

\section{References}

[1] J. Parker, P. Claesson, and P. Attard, Bubbles, cavities, and the long-ranged attraction between hydrophobic surfaces, J. Phys. Chem. 98, 8468 (1994).

[2] M. A. Hampton and A. V. Nguyen, Nanobubbles and the nanobubble bridging capillary force, Adv. Colloid Interface Sci. 154, 30 (2010).

[3] J. R. T. Seddon and D. Lohse, Nanobubbles and micropancakes: gaseous 
domains on immersed substrates, J. Phys.: Condens. Matter 23, 133001 (2011).

[4] V. S. J. Craig, Very small bubbles at surfaces-the nanobubble puzzle, Soft Matter 7, 40 (2011).

[5] J. R. T. Seddon, D. Lohse, W. A. Ducker, and V. S. J. Craig, A Deliberation on Nanobubbles at Surfaces and in Bulk, ChemPhysChem 13, 2179 (2012).

[6] S. Karpitschka, E. Dietrich, J. R. T. Seddon, H. J. W. Zandvliet, D. Lohse, and H. Riegler, Nonintrusive Optical Visualization of Surface Nanobubbles, Phys. Rev. Lett. 109, 066102 (2012).

[7] C. U. Chan and C.-D. Ohl, Total-Internal-Reflection-Fluorescence Microscopy for the Study of Nanobubble Dynamics, Phys. Rev. Lett. 109, 174501 (2012).

[8] B. M. Borkent, S. de Beer, F. Mugele, and D. Lohse, On the Shape of Surface Nanobubbles, Langmuir 26, 260 (2010).

[9] X. H. Zhang, N. Maeda, and V. S. J. Craig, Physical Properties of Nanobubbles on Hydrophobic Surfaces in Water and Aqueous Solutions, Langmuir 22, 5025 (2006).

[10] B. M. Borkent, S. M. Dammer, H. Schönherr, G. J. Vancso, and D. Lohse, Superstability of Surface Nanobubbles, Phys. Rev. Lett. 98, 204502 (2007).

[11] W. A. Ducker, Contact Angle and Stability of Interfacial Nanobubbles, Langmuir 25, 8907 (2009).

[12] M. P. Brenner and D. Lohse, Dynamic Equilibrium Mechanism for Surface Nanobubble Stabilization, Phys. Rev. Lett. 101, 214505 (2008).

[13] J. R. T. Seddon, H. J. W. Zandvliet, and D. Lohse, Knudsen Gas Provides Nanobubble Stability, Phys. Rev. Lett. 107, 116101 (2011).

[14] X. Zhang, M. H. Uddin, H. Yang, G. Toikka, W. Ducker, and N. Maeda, Effects of Surfactants on the Formation and the Stability of Interfacial Nanobubbles, Langmuir 28, 10471 (2012).

[15] S. Yang, P. Tsai, E. S. Kooij, A. Prosperetti, H. J. W. Zandvliet, and D. Lohse, Electrolytically Generated Nanobubbles on Highly Orientated Pyrolytic Graphite Surfaces, Langmuir 25, 1466 (2009). 
[16] X. Zhang, D. Y. C. Chan, D. Wang, and N. Maeda, Stability of Interfacial Nanobubbles, Langmuir 29, 1017 (2013).

[17] S. Yang, S. M. Dammer, N. Bremond, H. J. W. Zandvliet, E. S. Kooij, and D. Lohse, Characterization of Nanobubbles on Hydrophobic Surfaces in Water, Langmuir 23, 7072 (2007).

[18] S. Yang, E. S. Kooij, B. Poelsema, D. Lohse, and H. J. W. Zandvliet, Correlation between geometry and nanobubble distribution on HOPG surface, Europhys. Lett. 81, 64006 (2008).

[19] J. W. G. Tyrrell and P. Attard, Atomic Force Microscope Images of Nanobubbles on a Hydrophobic Surface and Corresponding Force-Separation Data, Langmuir 18, 160 (2002).

[20] X. H. Zhang, X. Zhang, J. Sun, Z. Zhang, G. Li, H. Fang, X. Xiao, X. Zeng, and J. Hu, Detection of Novel Gaseous States at the Highly Oriented Pyrolytic Graphite-Water Interface, Langmuir 23, 1778 (2007).

[21] L. Zhang, Y. Zhang, X. Zhang, Z. Li, G. Shen, M. Ye, C. Fan, H. Fang, and J. Hu, Electrochemically Controlled Formation and Growth of Hydrogen Nanobubbles, Langmuir 22, 8109 (2006). 


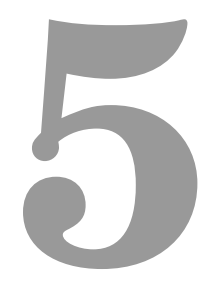

\section{Ultrasonically induced growth of surface nanobubbles: Experiment, numerics, and theory *}

In a recent study by Brotchie and Zhang [1] it was found that, when subjected to an acoustic field, surface nanobubbles can grow. It was postulated that this growth occurred due to rectified diffusion, similarly as with bulk (spherical) bubbles in an acoustic field. In this chapter, we review these experiments and provide a model (based on the diffusive model discussed in chapter 4) with which the effect of an acoustic field on surface nanobubbles, namely growth by rectified diffusion, is studied. Using the method of seperation of timescales, we recover a criterium for nanobubble growth due to rectified diffusion which we qualitatively compare to the experimental results. We establish a detailed phase diagram revealing how the bubble growth or shrinkage depends on the bubble size and driving parameters such as the amplitude of the acoustic signal.

*Manuscript in preparation: "Ultrasonically induced growth of surface nanobubbles: Experiment, numerics, and theory", J.H. Weijs, X.H. Zhang, K. van der Weelde, and D. Lohse. 


\subsection{Introduction}

Various recent studies have revealed the existence of nanoscale gas bubbles on liquid-solid interfaces. These objects, dubbed surface nanobubbles [2], typically have heights of $\sim 10 \mathrm{~nm}$ and diameters of $2 R_{b} \approx 100 \mathrm{~nm}$. As pointed out in reference [3], they are of potentially great technological importance, since they result in fluid slip on hydrophobic surfaces, thus reducing the drag in micro- and nanofluidics applications. The evidence in their favor is meanwhile considerable; for recent reviews see e.g. references [4-6]. While in the first decade after the discovery of surface nanobubbles [7] this evidence mainly came from atomic force microscopy (AFM) - a method which had been suspected to generate these nanobubbles - in the last years various other nonintrusive methods -from infrared spectroscopy $[8,9]$ to quartz crystal microbalance studies [10] to direct optical visualisation through optical interference-enhanced reflection microscopy [11] to total internal reflection fluorescence microscopy [12] to neutron $[13,14]$ and $x$-ray reflectivity [15]- have proven that these surface nanobubbles exist and can survive for days.

The key question is: why? Given their small size and their corresponding large Laplace pressure, one would expect a lifetime of only microseconds. Various theories have been suggested to account for the long stability of surface nanobubbles. Ducker [16] suggested that the origin lies in a coating of the bubbles with contaminants which would block the diffusive gas exchange. However, Das et al. [17] argued that this effect would not be sufficient to account for the experimentally observed lifetimes. Brenner and Lohse [18] and later Seddon et al. [19] suggested that the nanobubbles would survive due to a dynamic equilibrium, with the diffusive gas outflux being compensated by an influx close the contact line. However, neither experiments $[12,20]$ nor MD simulations [21] could provide evidence for such a flux. Finally, in chapter 4 and also in refs. $[22,23]$ it was suggested that the contact line pinning plays a prominent role in the stabilisation of the surface nanobubbles, reducing the Laplace pressure during dissolution and accounting for lifetimes up to tens of hours, as the relevant length scale would not be the bubble size, but the distance to the outside world (chapter 4), i.e., the thickness of the droplet or pool in which the nanobubbles live. However, there is also evidence against this hypothesis, at least in salty water: Berkelaar et al. [24] found that in a drying salt droplet, nanobubbles survive up to the very end of the drying process, i.e., when the liquid layer is so thin that according to the diffusive model in chapter 4 one would expect fast 
dissolution.

In view of this puzzling situation, the field is in need of controlled experiments for situations which are also theoretically or numerically accessible. An important control mechanism for gas bubbles with diffusive gas exchange with their environment is an acoustic field, defined by its amplitude $p_{a c}$ and its frequency $f$. It is well known that acoustically driving free gas bubbles can lead to their growth by rectified diffusion $[25,26]$ : Compressed bubbles lose gas due to their overpressure, but during the expansion phase for strong enough acoustic driving this loss can be compensated by a gas influx through the then larger bubble surface, leading to a net bubble growth. This effect could quantitatively be studied in the context of single bubble sonoluminescence (see e.g. refs. $[27,28]$ ), leading to quantitative agreement between theory and experiment [29].

Recently, Brotchie and Zhang [1] showed that also surface nanobubbles can grow by rectified diffusion. The aim of this study is to perform a numerical and theoretical analysis of acoustically driven surface nanobubbles, next to further analysis of the experimental data obtained in ref. [1] (provided in section 5.2). By doing so, we want to sharpen the modelling tools for surface nanobubbles, to eventually better understand their long lifetime also in the case without acoustic driving.

We will use a one-dimensional model, similar to that one developed in chapter 4, but now with an acoustic field included. This model is developed in section 5.3. In section 5.4 we numerically solve the model, and in section 5.5 we use the method of seperation of timescales (between the acoustic driving period and bubble growth timescale) to formulate a criterium for nanobubble growth. Finally, the results are compared to the experimental data.

\subsection{Experimental observations}

The setup for producing and imaging nanobubbles is shown in figure 5.1. This setup allows us to image the same nanobubbles before and after the application of ultrasound. The substrate, freshly cleaved highly oriented pyrolytic graphite (HOPG), was fixed onto the inside surface of the bottom of a closed fluid cell by epoxy. The outer surface of the cell bottom was glued firmly onto a piezoceromic ultrasound transducer (Undatim Ultrasonics, 515 $\mathrm{kHz}$ ). The top half of the cell was sealed by a flexible membrane, through which the AFM tip holder was mounted. There were two ports on the side 

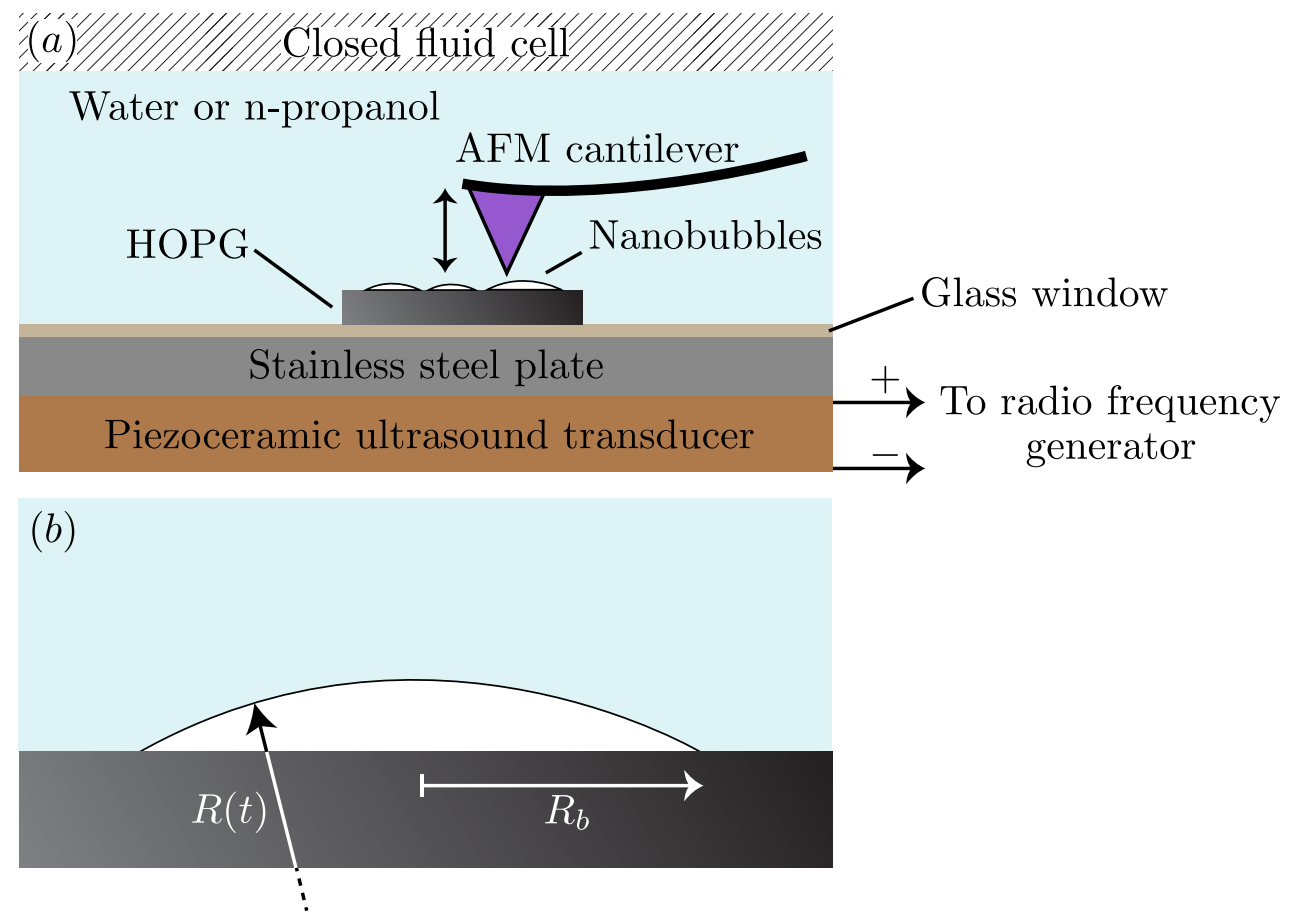

Figure 5.1: (a) Sketch of the nanobubble setup. The nanobubbles are created in a closed AFM liquid cell on a sample that is attached to a piezoceramic ultrasound transducer. (b) Close-up of a single nanobubble with (fixed) base radius $R_{b}$ and variable radius of curvature $R(t)$. 

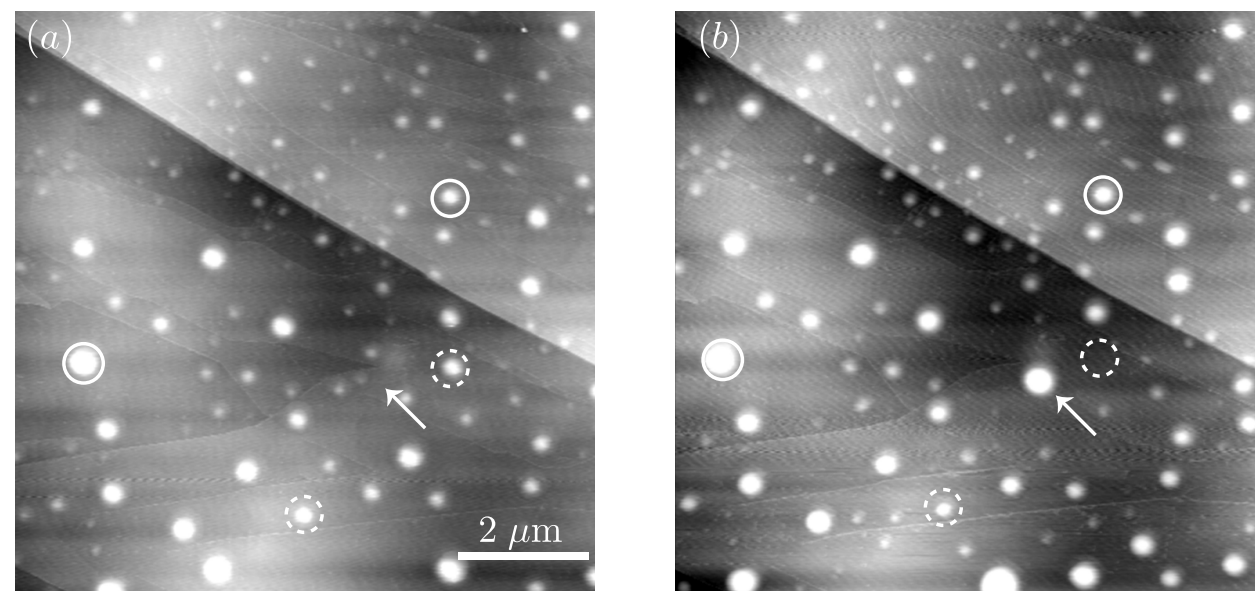

Figure 5.2: Tapping mode AFM height images of a collection of nanobubbles before (a) and after (b) having been exposed to a sound field for $40 \mathrm{~s}$. Some bubbles have been marked as a guide to the eye: solid circles indicate growing nanobubbles, dashed circles indicate shrinking or disappearing nanobubbles. The arrows indicate the location where a new nanobubble is formed, possibly out of a merger of several smaller nanobubbles.

body of the fluid cell as the inlet and outlet of solvents.

Surface nanobubbles were produced by an established method, called solvent exchange process. In this process, ethanol is first introduced into the fluid cell, and then thoroughly rinsed by water. The nanobubbles in this work were produced and imaged in a $100 \mathrm{mM}$ n-propanol solution. The ethanol used in the experiments was double distilled. Water was purified by a Milli-Q unit and stored at $4^{\circ} \mathrm{C}$ overnight to be saturated with air. Before use, it was slowly warmed up to $37^{\circ} \mathrm{C}$, leading to gas supersaturation.

Nanobubbles were imaged by tapping mode AFM (MFP-3D, Asylum Research, Santa Barbara, CA). The cantilever (NP, Bruker) with a normal spring constant of $0.32 \mathrm{~N} / \mathrm{m}$ was cleaned by UV/ozone irradiation for 15-20 min before use. The location of the AFM tip on the substrate was recorded by a CCD camera and the cleavage steps on HOPG that were visible optically could be used as the reference to assist the relocation of the AFM tip in the later measurements after the sonication.

Once the AFM images of nanobubbles were obtained in water, the AFM tip was withdrawn at least $100 \mu \mathrm{m}$ from the substrate and the ultrasound was applied to the system. The ultrasound was terminated after 25-6o s, imme- 


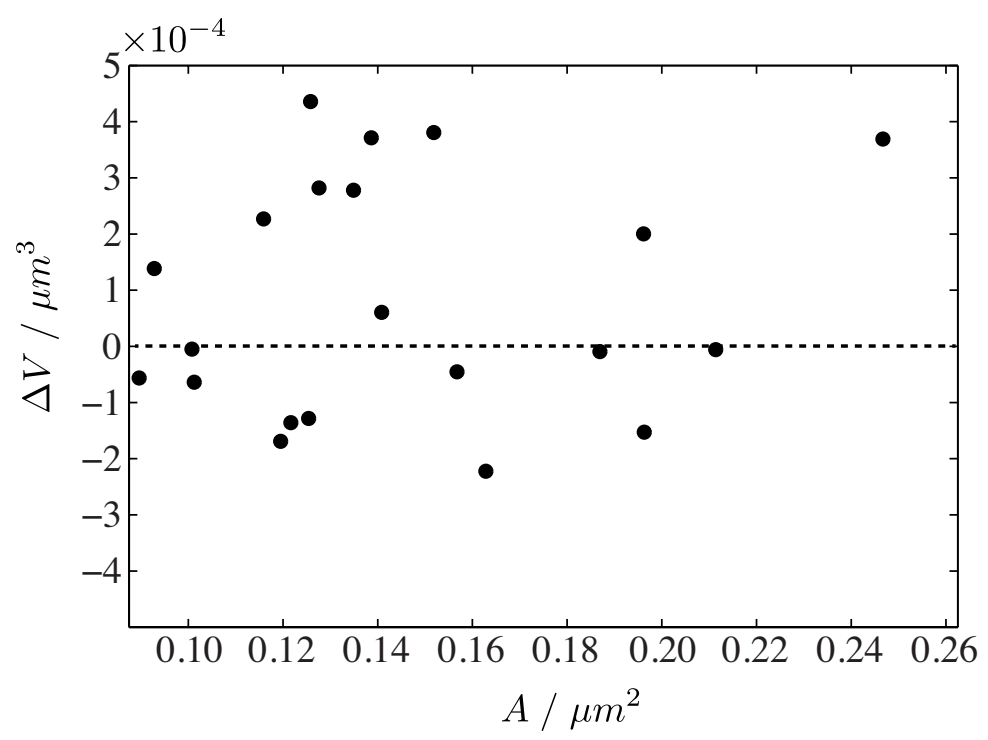

Figure 5.3: Absolute volume change of a selection of the bubbles from Fig. 5.2. The influence of the ultrasound is quite pronounced: typical nanobubble volumes are $10^{-3} \mu \mathrm{m}^{3}$, so the relative volume changes are $10-50 \%$. Note that some bubbles shrink, as also observed in Fig. 5.2.

diately upon the formation of micrometer-sized bubbles that arose through cavitation, regardless of the presence or absence of surface nanobubbles. The ultrasonic intensity transmitted to the fluid cell was estimated calorimetrically to be $2.2 \mathrm{~W}$ and could lead to a temperature increase of about $4 \mathrm{~K}$. The system was allowed to cool down to the temperature as before the sonication, and then the AFM tip was brought close to the substrate and relocated to the same imaging area as before the sonication. The images of the same nanobubbles were taken by using the same imaging parameters (e.g. set point, drive frequency and drive amplitude).

\subsection{Mathematical formulation of the problem}

We set out to calculate the time evolution of the gas concentration field $c$ which denotes the number concentration $\left([c]=\# / m^{3}\right)$. We assume, to make the analysis tractable, that diffusion mainly takes place in the $z$-direction (normal to the solid-liquid interface), similarly as done in chapter 4 where it 
was found that this is a valid assumption due to the large nanobubble density on the surface. Furthermore, we apply the flat bubble approximation (as in ref. [18]), meaning that during bubble oscillations no liquid is displaced and only the liquid-gas interfacial area $A_{b u b}(t)$ changes. Thus, $c(z, t)$ is assumed to obey the one-dimensional diffusion equation:

$$
\frac{\partial c}{\partial t}=D \frac{\partial^{2} c}{\partial z^{2}} .
$$

The bubble at the bottom of the container $(z=0)$ undergoes oscillations due to the driving pressure signal. In the far field $(z \rightarrow \infty)$ the concentration of dissolved gas is taken to have the constant value $c_{\infty}$. So we have:

$$
\begin{aligned}
c(z=0, t) & =\frac{p_{b u b}(t)}{k_{H}}=\frac{1}{k_{H}}\left(p_{a m b}+p_{a c} \sin \omega t+\frac{2 \gamma}{R(t)}\right), \\
c(z \rightarrow \infty, t) & =c_{\infty},
\end{aligned}
$$

with $p_{a c}$ and $\omega$ the amplitude and driving frequency of the acoustic signal, respectively, and $R(t)$ the radius of curvature of the bubble surface; $p_{a m b}$ is the ambient pressure in the undisturbed liquid. Usually this will be equal to atmospheric pressure but in a liquid cell with a closed lid it may also be different.

Since we apply a periodic pressure signal $p_{a c} \sin (\omega t)$ the pressure in the bubble $p_{b u b}(t)$ will vary in a periodic fashion. In the above equation we have assumed that it does so without any time delay. The pressure $p_{b u b}(t)$ also depends on the radius of curvature, via the Laplace term $2 \gamma / R(t)$, where $\gamma$ is the surface tension. Also $R(t)$ will vary periodically.

The radius of curvature depends, among other things, on the number $N(t)$ of gas molecules in the bubble which changes according to:

$$
\frac{\mathrm{d} N}{\mathrm{~d} t}=\left.D A_{b u b}(t) \frac{\partial c}{\partial z}\right|_{z=0} .
$$

The exact dependence of the bubble shape on the pressure in the ambient liquid is described in the next section.

The first step is to nondimensionalize above equations. We rescale all distances in the problem with the base radius $R_{b}$ of the bubble, so (denoting dimensionless variables by a tilde) $\tilde{z}=z / R_{b}, \tilde{A}=A / R_{b}^{2}$, etc. The time is made dimensionless via $\tilde{t}=\omega t$ and the concentration via $\tilde{c}=c / c_{\infty}$. With these choices [which also fix the dimensionless form of all other quantities 
in the system e.g. $\tilde{p}=p c_{\infty} k_{H}$, and provides the proper way to rescale $N$ : $\left.\tilde{N}=N /\left(R_{b}^{3} c_{\infty}\right)\right]$ we find that Eqs. (5.1) and (5.4) take the form:

$$
\begin{aligned}
\frac{\partial \tilde{c}}{\partial \tilde{t}} & =\epsilon \frac{\partial^{2} \tilde{c}}{\partial \tilde{z}^{2}}, \\
\frac{\mathrm{d} \tilde{N}}{\mathrm{~d} \tilde{t}} & =\left.\epsilon \tilde{A}(\tilde{t}) \frac{\partial \tilde{c}}{\partial \tilde{z}}\right|_{\tilde{z}=0}
\end{aligned}
$$

with $\epsilon=1 / \mathrm{Pe} \ll 1$, where $\mathrm{Pe}=\omega R_{b}^{2} / D$ is the Péclet number. In a typical experiment (with $\omega=2 \pi f=3 \cdot 10^{6} \mathrm{rad} \cdot \mathrm{s}^{-1}, R_{b}=10^{-7} \mathrm{~m}$ and $D=2 \cdot 10^{-9} \mathrm{~m}^{2} / \mathrm{s}$ ) the value of the small parameter is $\epsilon=0.06$. The boundary conditions (5.2) and (3) in dimensionless form are:

$$
\begin{aligned}
\tilde{c}(\tilde{z}=0, \tilde{t}) & =\tilde{p}_{a m b}+\tilde{p}_{a c} \sin \tilde{t}+\tilde{p}_{L}(\tilde{t}), \\
\tilde{c}(\tilde{z} \rightarrow \infty, \tilde{t}) & =1,
\end{aligned}
$$

where $\tilde{p}_{L}$ denotes the non-dimensionalized Laplace pressure.

\subsection{Numerical evaluation}

In this section we solve the diffusion equation (5.1) with boundary conditions (5.2) numerically. We will find that, under favourable conditions, the nanobubbles indeed grow under the influence of an ultrasonic field, as in the experiments. By looking at the obtained concentration profiles $c(z, t)$ we can identify important properties which we will use when deriving an analytic solution in section $5 \cdot 5$.

\subsubsection{Numerical procedure and nanobubble geometry}

To numerically evaluate the diffusion equation (5.1) we define a non-uniform grid in $z$, such that the large gradients in $c$ near $z=0$ (due to the fast bubble oscillations) are captured while allowing to evaluate large system sizes by using a coarser grid far away from the bubble, where the concentration profile is much smoother. The partial differential equation (5.1) is discretized on this grid to a system of initial value ordinary differential equations, which are then solved using an explicit, variable time step, Runge-Kutta (DormandPrice method) scheme. The timestep is chosen such that during each driving cycle, the equations are resolved at least six times, and at all timesteps the boundary condition at $z=0$ is explicitly calculated. It is at this boundary 
$(z=0)$ where the effects of the ultrasound enters the equations, as the shape and internal pressure of the bubbles are directly affected by the ultrasound signal. Equation (5.4) is solved in parallel and is affected by the local density gradient $\partial c / \partial z$ at $z=0$ and also couples back through the boundary condition at $z=0$, equation (5.2).

Finally, to close the problem we require a relation between the pressure in the liquid (which is typically the atmospheric pressure plus the (ultrasonic) driving pressure) and the shape of the bubble at given $N(t)$ and $\gamma$. For the gas, we assume the van der Waals equation of state $p_{b u b} \cdot(V-N b)=N k_{B} T$ to hold, where $p_{b u b}$ is the absolute pressure inside the bubble, $V$ the volume of the bubble, $N$ the number of atoms inside the bubble, $b$ the excluded volume due to the finite size of the atoms, $k_{B}$ Boltzmann's constant, and $T$ the temperature (constant at $300 \mathrm{~K})$. The equation describes an ideal gas, with a correction $b N$ to the volume to account for the fact that a bubble cannot be compressed to zero volume due to the finite size of the atoms. The bubble shape is described by a spherical cap, and we assume that the nanobubbles are pinned, i.e. their contact lines do not move, but the contact angle $\theta$ is allowed to vary. Previously, in chapter 4, we showed that the lifetime of pinned nanobubbles is the same as those observed in experiments. Furthermore, the pinned contact line explains many other special features of nanobubbles such as their low contact angles (compared to Young's law), their resilience to Ostwald ripening, and their 'superstability' against tensile stress shocks [30]. Additionally, there is experimental evidence that the contact line of nanobubbles is not round [31] and that the dimensions of growing and shrinking nanobubbles vary mostly in height alone, rather than both height and width as would be the case for unpinned bubbles. Furthermore, recent optical measurements of a meniscus retracting over surface nanobubbles clearly show pinning effects: the meniscus becomes curved as it moves over a nanobubble [32].

\subsubsection{Numerical results}

In figure 5.4 the nanobubble content $N(t)$ is shown as a function of time for two cases. Figure 5.4(a) shows the gas content of a nanobubble in an acoustic field. In this case, the acoustic driving is insufficient to let the nanobubble grow and it shrinks. In figure $5.4(\mathrm{~b})$, with the same acoustic driving $\left(p_{a c}=1.5 \mathrm{~atm}\right)$ but a larger bubble $\left(R_{b}=1 \mu \mathrm{m}\right)$ we see that the bubble grows due to rectified diffusion. It is therefore clear that in order to achieve growth by rectified diffusion, certain conditions have to be fulfilled and it 

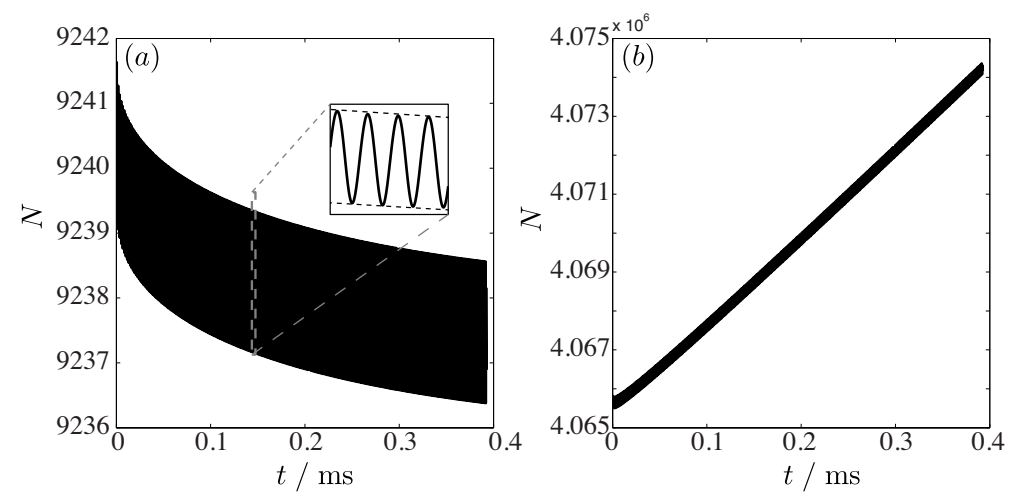

Figure 5.4: Nanobubble content $N$ as a function of time $t$ for a nanobubble with the following parameters: $p_{a c}=1.5 \mathrm{~atm}, \gamma=0.05 \mathrm{~J} / \mathrm{m}^{2}, c_{\infty}=p_{a t m} / k_{H}$, and $\theta_{0}=10^{\circ}$. (a) Small bubble, $R_{b}=100 \mathrm{~nm}$. Although there is some mass-gain due to rectified diffusion, this is not enough to balance the diffuse outflux under these particular conditions, so the bubble shrinks. The inset shows some details where clearly the seperation between the fast timescale (oscillations due to the driving signal) and the slow timescale (net shrinkage of the bubble) can be seen. (b) Large bubble, $R_{b}=1 \mu \mathrm{m}$. In this case, the driving pressure of the acoustic signal is strong enough to overcome diffusive outflux by rectified diffusion and the bubble grows.

is the primary goal of this chapter to form a detailed phase diagram for nanobubble growth by rectified diffusion.

In figure 5.5 the concentration profile is shown at different times for the case of a bubble that grows by rectified diffusion. The inset shows a zoom around $z=0$ where the oscillatory behaviour of the bubble is clearly visible for $z<\delta$, with $\delta \sim \sqrt{D / \omega}$. Outside this region $(z>\delta)$ a much smoother profile is recovered as all high frequency oscillations caused by the rapidly expanding and shrinking bubble are damped out. It will turn out that the shape of this smooth profile determines whether nanobubbles grow or shrink.

\subsection{Analytical derivation of the bubble growth rate}

\section{Separation of time-scales}

To solve the set of equations (5.5) with boundary conditions (5.7) analytically, we make use of the fact that there are two distinct timescales in this problem: (i) the fast timescale $\tilde{t}$ of the pressure oscillations and (ii) the slow 


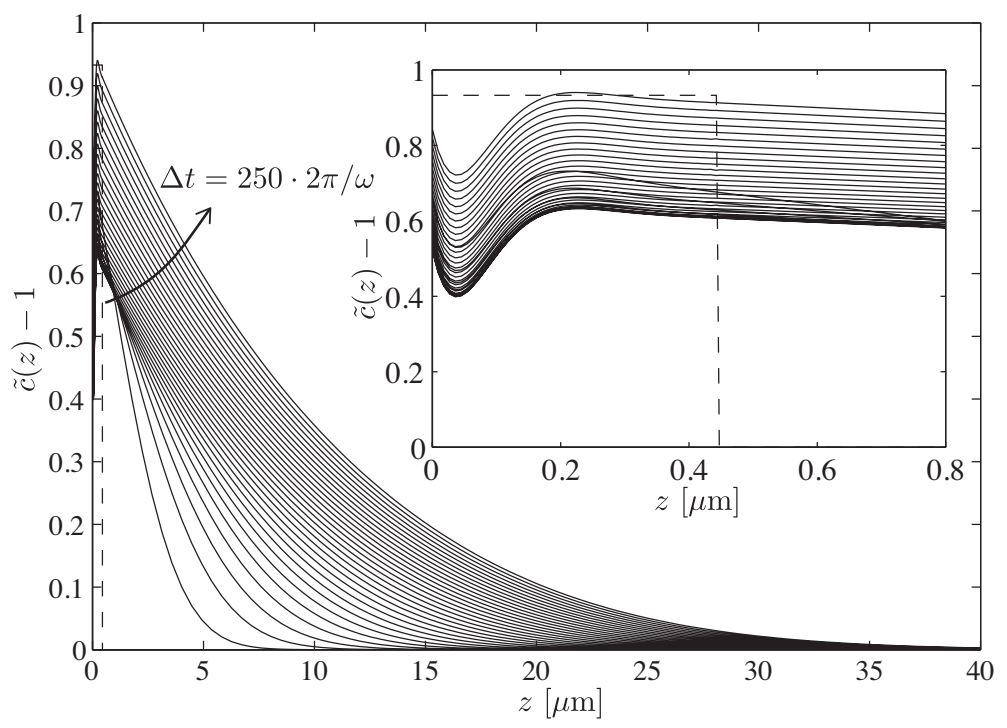

Figure 5.5: Concentration profiles of dissolved gas calculated numerically using the method described in section 5.4, in the case of a growing bubble. The dashed line represents the initial condition, where a small (approx. $0.4 \mu \mathrm{m}$ ) region of liquid above the nanobubble is supersaturated with gas. The solid lines give the concentration profiles with an interval of 250 driving cycles. Already before the first 250 cycles, the concentration profile assumes a shape which it preserved throughout the simulation: a smooth decay towards $c=c_{\infty}$ for large $z$, and at a very small scale $(z<0.2 \mu \mathrm{m})$ some nontrivial shape due to the oscillating bubble. Note that this oscillatory part of the concentration profile changes shape at the driving rate, but this cannot be seen in this plot due to the stroboscopic effect: all profiles shown are such that $(t \bmod 2 \pi / \omega)=0$. In order to simulate an infinite liquid, the numerical domain of $z$ extends beyond that shown in the plot here. 
diffusive timescale $\mathcal{T}=\epsilon \tilde{t}$ on which the bubble grows or shrinks. We employ a method similar to that used by Fyrillas and Szeri [27] and split the concentration profile into a fast, oscillatory part $\tilde{c}_{o s c}(\tilde{z}, \tilde{t})$ and a slow, smooth part $\tilde{c}_{s m}(\tilde{z}, \tilde{t})$ such that $\tilde{c}=\tilde{c}_{o s c}+\tilde{c}_{s m}$. The main idea in ref. [27] is to reformulate the problem such that the net gain or loss of the bubble only manifests itself in the smooth part of the solution and, consequently, the gain or loss in the oscillatory part over one period is zero. To achieve this, we need a non-linear time variable $\tau$ defined as follows:

$$
\tau(\tilde{t}) \equiv \int_{0}^{\tilde{t}} \tilde{A}^{2}\left(\tilde{t}^{\prime}\right) \mathrm{d} \tilde{t}^{\prime}
$$

We use the ^symbol throughout to indicate a nondimensional variable which depends on $\tau$ instead of $\tilde{t}$. We also split the boundary conditions into an oscillatory and a smooth part:

$$
\begin{aligned}
\hat{c}(\tilde{z}=0, \tau) & =\left\langle\hat{p}_{b u b}(\tau)\right\rangle_{\tau}+\left[\hat{p}_{b u b}(\tau)-\left\langle\hat{p}_{b u b}(\tau)\right\rangle_{\tau}\right], \\
\hat{c}(\tilde{z} \rightarrow \infty, \tau) & =1+0 .
\end{aligned}
$$

where $\langle\cdot\rangle_{\tau}$ denotes the (weighed) temporal average over one oscillation pe$\operatorname{riod} T_{a c}=2 \pi / \omega$ :

$$
\langle f(\tilde{t})\rangle_{\tau}=\frac{\int_{0}^{T_{a c}} f(\tilde{t}) \tilde{A}^{2}(\tilde{t}) \mathrm{d} \tilde{t}}{\int_{0}^{T_{a c}} \tilde{A}^{2}(\tilde{t}) \mathrm{d} \tilde{t}}
$$

\section{Oscillatory part}

We first deal with the oscillatory part of the problem. The problem is now posed as follows:

$$
\begin{gathered}
\frac{\partial \tilde{c}_{o s c}}{\partial \tilde{t}}=\epsilon \frac{\partial^{2} \tilde{c}_{o s c}}{\partial \tilde{z}^{2}}, \\
\frac{\mathrm{d} \tilde{N}_{o s c}}{\mathrm{~d} \tilde{t}}=\left.\epsilon \tilde{A}(\tilde{t}) \frac{\partial \tilde{c}_{o s c}}{\partial \tilde{z}}\right|_{\tilde{z}=0},
\end{gathered}
$$

with the boundary conditions as indicated in (5.9) and (5.10). By replacing the spatial coordinate $\tilde{z}$ by the rescaled coordinate $s=\tilde{A}(\tilde{t}) \tilde{z}$ (such that $\partial \tilde{c} / \partial \tilde{z}=\tilde{A} \partial \tilde{c} / \partial s$ and $\left.\partial^{2} \tilde{c} / \partial z^{2}=\tilde{A}^{2} \partial^{2} \tilde{c} / \partial s^{2}\right)$ the above two equations are recast into a form that contains $\tilde{A}^{2}(\tilde{t})$ on the right hand side of both equations. Transferring this time-dependent factor to the time derivative, with $\tilde{A}^{2}(\tilde{t}) d \tilde{t}=d \tau$ [which explains our choice for the non-linear time $\tau$ introduced 
in Eq. (5.8)] we get for the diffusion and mass gain equations for $\hat{c}_{o s c}(s, \tau)$ and $\hat{N}(s, \tau)$ :

$$
\begin{aligned}
& \frac{\partial \hat{c}_{o s c}}{\partial \tau}=\epsilon \frac{\partial^{2} \hat{c}_{o s c}}{\partial s^{2}}, \\
& \frac{\mathrm{d} \hat{N}}{\mathrm{~d} \tau}=\left.\epsilon \frac{\partial \hat{c}_{o s c}}{\partial s}\right|_{s=0} .
\end{aligned}
$$

We see how the spatial and temporal transformations described above have resulted into a set of equations without any dependence on the varying bubble area $\tilde{A}$. From this, it can be inferred that the net mass gain over a complete oscillation period must necessarily be zero. This becomes explicit by deriving the full solution of the oscillatory problem, which we derive below.

First, we decompose the boundary condition into a Fourier series:

$$
\begin{aligned}
& \hat{c}_{o s c}(s=0, \tau)=\hat{p}_{b u b}(\tau)-\left\langle\hat{p}_{b u b}(\tau)\right\rangle_{\tau} \\
&=\sum_{m=0}^{\infty}\left[a_{m} \cos \omega_{m} \tau+b_{m} \sin \omega_{m} \tau\right],
\end{aligned}
$$

with

$$
\omega_{m}=\frac{2 m \pi}{\tau\left(T_{a c}\right)}=\frac{2 m \pi}{\int_{0}^{T_{a c}} \tilde{A}^{2}(\tilde{t}) \mathrm{d} \tilde{t}} .
$$

Note that $a_{0}=0$, since it corresponds to the mean value, which is zero:

$$
\left\langle\hat{c}_{o s c}(s=0, \tau)\right\rangle_{\tau}=\left\langle\hat{p}_{b u b}(\tilde{t})\right\rangle_{\tau}-\left\langle\left\langle\hat{p}_{b u b}(\tau)\right\rangle_{\tau}\right\rangle_{\tau}=0 .
$$

We can now solve the oscillatory problem for each Fourier mode $m \geq 1$ to get:

$$
\begin{gathered}
\hat{c}_{o s c}(s, \tau)=\sum_{m=1}^{\infty}\left(\exp \left[-\left(\frac{\omega_{m}}{2}\right)^{\frac{1}{2}} s\right] \times\right. \\
\left.\left\{a_{m} \cos \left(\epsilon \omega_{m} \tau-\left(\frac{\omega_{m}}{2}\right)^{\frac{1}{2}} s\right)+b_{m} \sin \left(\epsilon \omega_{m} \tau-\left(\frac{\omega_{m}}{2}\right)^{\frac{1}{2}} s\right)\right\}\right) .
\end{gathered}
$$

The bubble growth over a single cycle associated with the oscillatory part of the problem is therefore identically zero:

$$
\begin{gathered}
\int_{0}^{\tau\left(T_{a c}\right)} \frac{\mathrm{d} \hat{N}}{\mathrm{~d} \tau^{\prime}} \mathrm{d} \tau=\left.\int_{0}^{\tau\left(T_{a c}\right)} \epsilon R_{b}^{3} c_{\infty} \frac{\partial \hat{c}}{\partial s}\right|_{s=0} \mathrm{~d} \tau= \\
\epsilon R_{b}^{3} c_{\infty} \int_{0}^{\tau\left(T_{a c}\right)} \sum_{m=1}^{\infty}\left(\frac{\omega_{m}}{2}\right)^{\frac{1}{2}}\left[a_{m}\left(\sin \left(\epsilon \omega_{m} \tau\right)-\cos \left(\epsilon \omega_{m} \tau\right)\right)\right. \\
\left.+b_{m}\left(\sin \left(\epsilon \omega_{m} \tau\right)-\cos \left(\epsilon \omega_{m} \tau\right)\right)\right] \mathrm{d} \tau=0
\end{gathered}
$$

This is a the desired result: by scaling the time and space variables with the fluctuating area of the bubble, we have managed to move the entire mass flux towards the smooth solution. 


\section{Smooth part}

The next step is to solve the smooth concentration profile. In this step we will also need the 'regular' time average:

$$
\langle f(\tilde{z}, \tilde{t})\rangle_{\tilde{t}} \equiv \frac{1}{T_{a c}} \int_{0}^{T_{a c}} f(\tilde{z}, \tilde{t}) \mathrm{d} \tilde{t}
$$

and we define the slow, non-dimensional timescale

$$
\mathcal{T} \equiv \epsilon \tilde{t}=\frac{t D}{R_{b}^{2}} .
$$

It is instructive to compare the two timescales. The fast time $\tilde{t}$ was nondimensionalized with the driving frequency $\omega$. The events at this timescale affect the concentration profile only in a small region near the bubble, see Eq. (5.19). The slow timescale $\mathcal{T}$ represents slow diffusive effects. To achieve adequate separation between the timescales, which is required to split the oscillatory and smooth parts, $\epsilon=1 /$ Pe must be sufficiently small, as evidenced by the definition of $\mathcal{T}(5.22)$. We recall the equation to solve:

$$
\frac{\partial \tilde{c}_{s m}}{\partial \tilde{t}}=\epsilon \frac{\partial^{2} \tilde{c}_{s m}}{\partial \tilde{z}^{2}}
$$

and expand $\tilde{c}_{s m}(\tilde{z}, \tilde{t})$ as follows:

$$
\tilde{c}_{s m}(\tilde{z}, \tilde{t})=\tilde{c}_{s m}^{0}(\tilde{z}, \tilde{t}, \mathcal{T})+\epsilon \tilde{c}_{s m}^{1}(\tilde{z}, \tilde{t}, \mathcal{T})+\mathcal{O}\left(\epsilon^{2}\right) .
$$

With $\partial / \partial \tilde{t} \rightarrow \partial / \partial \tilde{t}+\epsilon \partial / \partial \mathcal{T}^{\dagger}$. Eq. (5.23) then becomes:

$$
\begin{aligned}
& \frac{\partial}{\partial \tilde{t}}\left(\tilde{c}_{s m}^{0}+\epsilon \tilde{c}_{s m}^{1}+\mathcal{O}\left(\epsilon^{2}\right)\right) \\
& \quad+\epsilon \frac{\partial}{\partial \mathcal{T}}\left(\tilde{c}_{s m}^{0}+\epsilon \tilde{c}_{s m}^{1}+\mathcal{O}\left(\epsilon^{2}\right)\right) \\
& \quad=\epsilon \frac{\partial^{2}}{\partial \tilde{z}^{2}}\left(\tilde{c}_{s m}^{0}+\epsilon \tilde{c}_{s m}^{1}+\mathcal{O}\left(\epsilon^{2}\right)\right) .
\end{aligned}
$$

To leading order $\left(\epsilon^{0}\right)$ this simply yields:

$$
\frac{\partial \tilde{c}_{s m}^{0}}{\partial \tilde{t}}=0,
$$

\footnotetext{
${ }^{\dagger}$ As described in ref. [33]. In this description $\partial / \partial \tilde{t}$ is taken at constant $\mathcal{T}$ and $\partial / \partial \mathcal{T}$ is taken with all quantities that depend on the fast time-scale $\tilde{t}$ averaged over one complete driving period.
} 
which tells us that $\tilde{c}_{s m}^{0}$ does not depend on the fast time variable $\tilde{t}$ :

$$
\tilde{c}_{s m}^{0}(\tilde{z}, \tilde{t}, \mathcal{T})=\tilde{c}_{s m}^{0}(\tilde{z}, \mathcal{T}) .
$$

The dependence of $\tilde{c}_{s m}^{0}$ on the slow time variable $\mathcal{T}$ and $\tilde{z}$ will follow from the next order equation $\left(\epsilon^{1}\right)$, which reads:

$$
\frac{\partial \tilde{c}_{s m}^{1}}{\partial \tilde{t}}+\frac{\partial \tilde{c}_{s m}^{0}}{\partial \mathcal{T}}=\frac{\partial^{2} \tilde{c}_{s m}^{0}}{\partial \tilde{z}^{2}} .
$$

To exclude divergence of the expansion (secular behavior) we impose that $\left\langle\partial \tilde{c}_{s m}^{1} / \partial \tilde{t}\right\rangle_{\tilde{f}}=0$, which means that the above equation reduces to an equation for $\tilde{c}_{s m}^{0}(\tilde{z}, \mathcal{T})$ alone:

$$
\frac{\partial \tilde{c}_{s m}^{0}}{\partial \mathcal{T}}=\frac{\partial^{2} \tilde{c}_{s m}^{0}}{\partial \tilde{z}^{2}}
$$

where both sides are averaged over one driving cycle. This averaging does not have to be written explicitly, since these terms are independent of $\tilde{t}$ anyway (they do not change on the fast timescale). The corresponding boundary conditions are:

$$
\begin{aligned}
\tilde{c}_{s m}^{0}(\tilde{z}=0, \mathcal{T}) & =\left\langle\hat{p}_{b u b}(\tau)\right\rangle_{\tau}(\mathcal{T}) \\
\tilde{c}_{s m}^{0}(\tilde{z} \rightarrow \infty, \mathcal{T}) & =1 .
\end{aligned}
$$

The above problem is a diffusion equation with a time-dependent boundary condition at $\tilde{z}=0:\left\langle\hat{p}_{b u b}\right\rangle_{\tau}(\mathcal{T})$.

The full solution for this problem is [34]:

$$
\tilde{c}_{s m}^{0}(\tilde{z}, \mathcal{T})=1+\int_{0}^{\mathcal{T}}\left(\frac{\tilde{z}\left(\left\langle\hat{p}_{b u b}\right\rangle_{\tau}\left(\mathcal{T}^{\prime}\right)-1\right)}{\sqrt{4 \pi\left(\mathcal{T}-\mathcal{T}^{\prime}\right)^{3}}} \exp \left[-\frac{\tilde{z}^{2}}{4\left(\mathcal{T}-\mathcal{T}^{\prime}\right)}\right]\right) \mathrm{d} \mathcal{T}^{\prime}
$$

An approximate solution, assuming for the moment $\left\langle p_{b u b}\right\rangle_{\tau}$ to be constant, then gives:

$$
\tilde{c}_{s m}^{(0,0)}(\tilde{z}, \mathcal{T})=1+\left(\left\langle\hat{p}_{b u b}\right\rangle_{\tau}-1\right) \cdot \operatorname{Erfc}\left(\frac{\tilde{z}}{2 \sqrt{\mathcal{T}}}\right)
$$

where $\operatorname{Erfc}(\cdot)$ is the complimentary errorfunction $1-\operatorname{Erf}(\cdot)$. In figure 5.6 the same concentration profiles are plotted as those from the growing bubble in figure 5.5, nondimensionalized according to eq. (5.33). From this we clearly 


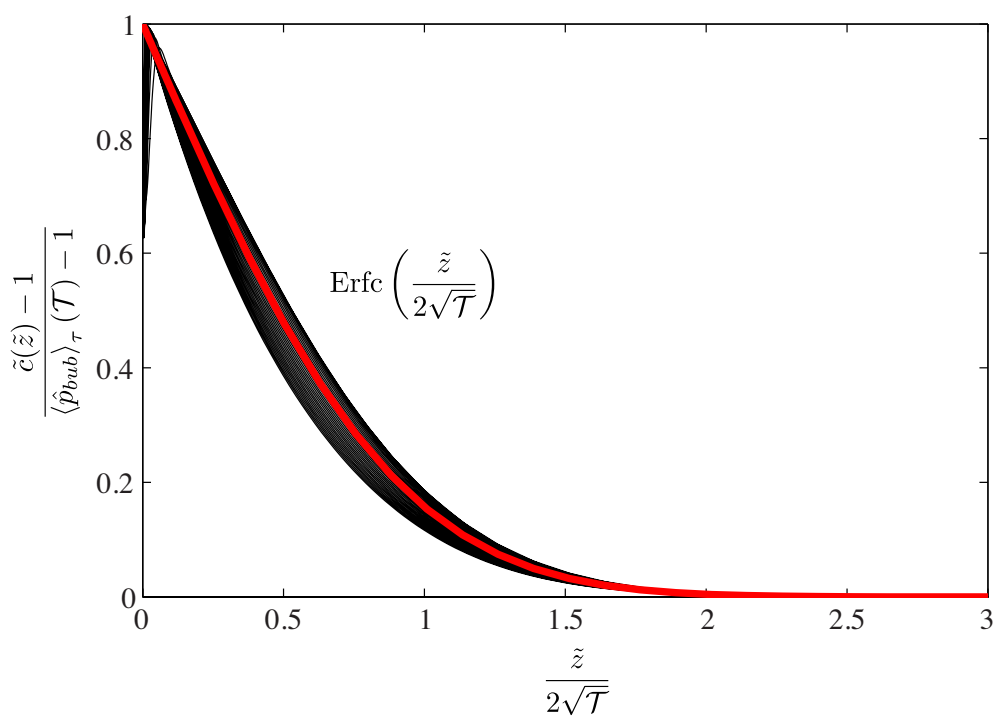

Figure 5.6: Nondimensionalized concentration profiles (based on equation (5.33)). The plot shows the same data as that in figure 5.5, which clearly collapses onto the approximated curve (5.33) (indicated by the red line). The small deviations (mainly at intermediate $\tilde{z} / \sqrt{4 \mathcal{T}}$ ) arise due to the fact that $\left\langle p_{b u b}\right\rangle_{\tau}$ is not completely constant in time. It shows, therefore, that the value of $\left\langle p_{b u b}\right\rangle_{\tau}$ indeed varies slowly, and a small error is to be expected due to this assumption. 
see that the approximate smooth solution (5.33) desbribes the numerical results very well: all profiles collapse on the predicted curve, indicated by the red line. The small deviations at intermediate $\tilde{z} / \sqrt{4 \mathcal{T}}$ are caused by the time-dependence of $\left\langle p_{b u b}\right\rangle_{\tau}$, which we have ignored in(eq. (5.33).

To evaluate the bubble growth rate we fully evaluate eq. (5.32). Simply taking the derivative with respect to $\tilde{z}$ will result in singular behaviour of the gradient at $\tilde{z}=0$, so a different approach is required. In order to do so, we first recognize that eq. (5.32) is a convolution, which is commutative, and we can exchange $\mathcal{T}^{\prime}$ and $\left(\mathcal{T}-\mathcal{T}^{\prime}\right)$. Naturally, this also follows from a substitution of variables. Doing so, and integrating by parts then gives:

$$
\begin{aligned}
\tilde{c}_{s m}^{0}(\tilde{z}, \mathcal{T})= & 1+\left.\operatorname{erf}\left(\frac{\tilde{z}}{2 \sqrt{\mathcal{T}^{\prime}}}\right) \cdot\left(\left\langle\hat{p}_{b u b}\right\rangle_{\tau}\left(\mathcal{T}-\mathcal{T}^{\prime}\right)-1\right)\right|_{\mathcal{T}^{\prime}=0} ^{\mathcal{T}^{\prime}=\mathcal{T}}- \\
& \int_{0}^{\mathcal{T}} \operatorname{erf}\left(\frac{\tilde{z}}{2 \sqrt{\mathcal{T}^{\prime}}}\right) \frac{\mathrm{d}}{\mathrm{d} \mathcal{T}^{\prime}}\left(\left\langle\hat{p}_{b u b}\right\rangle_{\tau}\left(\mathcal{T}-\mathcal{T}^{\prime}\right)-1\right) \mathrm{d} \mathcal{T}^{\prime}= \\
& 2+\operatorname{erf}\left[\frac{\tilde{z}}{2 \sqrt{\mathcal{T}}}\right]\left(\left\langle\hat{p}_{b u b}\right\rangle_{\tau}(0)-1\right)-\left\langle\hat{p}_{b u b}\right\rangle_{\tau}(\mathcal{T})- \\
& \int_{0}^{\mathcal{T}} \operatorname{erf}\left(\frac{\tilde{z}}{2 \sqrt{\mathcal{T}^{\prime}}}\right) \frac{\mathrm{d}}{\mathrm{d} \mathcal{T}^{\prime}}\left(\left\langle\hat{p}_{b u b}\right\rangle_{\tau}\left(\mathcal{T}-\mathcal{T}^{\prime}\right)-1\right) \mathrm{d} \mathcal{T}^{\prime},
\end{aligned}
$$

where we recognize the approximate solution (5.33).

The next step is to derive how the mass gain or loss rate depends on this solution. First, we take the regular time-average over one cycle at both sides of Eq. (5.14):

$$
\left\langle\frac{\partial \tilde{N}}{\partial \tau}\right\rangle_{\tilde{t}}=\epsilon\left(\left\langle\left.\frac{\partial \tilde{c}_{o s c}^{0}}{\partial s}\right|_{s=0}\right\rangle_{\tilde{t}}+\left\langle\left.\frac{\partial \tilde{c}_{s m}^{0}}{\partial s}\right|_{s=0}\right\rangle_{\tilde{t}}\right) .
$$

We know from the analysis of the oscillatory part of the solution that the term containing $\tilde{c}_{o s c}$ does not contribute, and we get after transforming $d \tau$ to $d \tilde{t}$ and $s$ to $\tilde{z}$ :

$$
\left\langle\frac{1}{\tilde{A}^{2}(\tilde{t}, \mathcal{T})} \frac{\mathrm{d} \tilde{N}}{\mathrm{~d} \mathcal{T}}\right\rangle_{\tilde{t}}=\left\langle\left.\frac{1}{\tilde{A}(\tilde{t}, \mathcal{T})} \frac{\partial \tilde{c}_{s m}^{0}}{\partial \tilde{z}}\right|_{\tilde{z}=0}\right\rangle_{\tilde{t}} .
$$

Since both partial derivatives in this expression depend on the slow timescale only they are constant over a single cycle and can be taken outside the $\langle\cdot\rangle_{\tilde{t}}$. We obtain:

$$
\frac{\mathrm{d} \tilde{N}}{\mathrm{~d} \mathcal{T}}=\left.\frac{\left\langle\tilde{A}^{-1}(\tilde{t}, \mathcal{T})\right\rangle_{\tilde{t}}}{\left\langle\tilde{A}^{-2}(\tilde{t}, \mathcal{T})\right\rangle_{\tilde{t}}} \frac{\partial \tilde{c}_{s m}^{0}}{\partial \tilde{z}}\right|_{\tilde{z}=0} .
$$


The derivative of $\tilde{c}_{s m}^{0}$ with respect to $\tilde{z}$ at $\tilde{z}=0$ is:

$$
\left.\frac{\partial \tilde{c}_{s m}^{0}}{\partial \tilde{z}}\right|_{\tilde{z}=0}=\frac{\left\langle\hat{p}_{b u b}\right\rangle_{\tau}(\mathcal{T}=0)-1}{\sqrt{\pi \mathcal{T}}}-\int_{0}^{\mathcal{T}} \frac{\frac{\mathrm{d}}{\mathrm{d} \mathcal{T}^{\prime}}\left(\left\langle\hat{p}_{b u b}\right\rangle_{\tau}\left(\mathcal{T}-\mathcal{T}^{\prime}\right)-1\right)}{\sqrt{\pi \mathcal{T}^{\prime}}} \mathrm{d} \mathcal{T}^{\prime} .
$$

The above integro-differential equation set (5.37), (5.38) is our desired result, which characterizes whether bubbles grow or shrink on the long timescale. Still, equations (5.37), (5.38) must be solved numerically, but this numerical integration is much less expensive than the brute-force integration of the diffusion equation.

\subsection{Phase space}

\section{Static behaviour}

From the previous section, specifically equations (5.29), (5.30), and (5.31), it is evident that bubbles grow by rectified diffusion when:

$$
\left\langle\hat{p}_{b u b}\right\rangle_{\tau}<1 \text {. }
$$

Note that the saturation concentration $c_{\infty}$ enters through the nondimensionalization of the pressure. The dependence of $\left\langle\hat{p}_{b u b}\right\rangle_{\tau}$ on $R_{b}$ and $p_{a c}$ is shown in Fig. 5.7, which because of (5.39) represents a phase diagram where we can differentiate between shrinking, growing, and cavitating bubbles for bubbles with $\theta=20^{\circ}$, at fixed $N$. The striped region indicates acoustic driving pressures that are so high that at some point during the driving cycle the bubbles will cavitate. The coloured iso-density lines indicate the parameter-range for which growth by rectified diffusion can occur, depending on the far-field concentration $c_{\infty}$, parametrized by:

$$
\eta \equiv \frac{c_{\infty} k_{H}}{p_{a t m}}
$$

We see that even in undersaturated solutions $(\eta<1)$, growth can occur for large enough $\left(R_{b} \gtrsim 1.5 \mu \mathrm{m}\right)$ bubbles and strong enough acoustic driving.

It is instructive to look at some limiting behaviour. First, we see that when $p_{a c}=0$ some bubbles grow. This can only occur in solutions that are supersaturated relative to the bubble pressure: $c_{\infty} \geq 2 \gamma /\left(R k_{H}\right)$ as there is no acoustic driving, and these are indeed the recovered values. For $R_{b} \rightarrow \infty$ the iso-lines corresponding to $\eta \leq 1$ therefore never reach the bottom of 


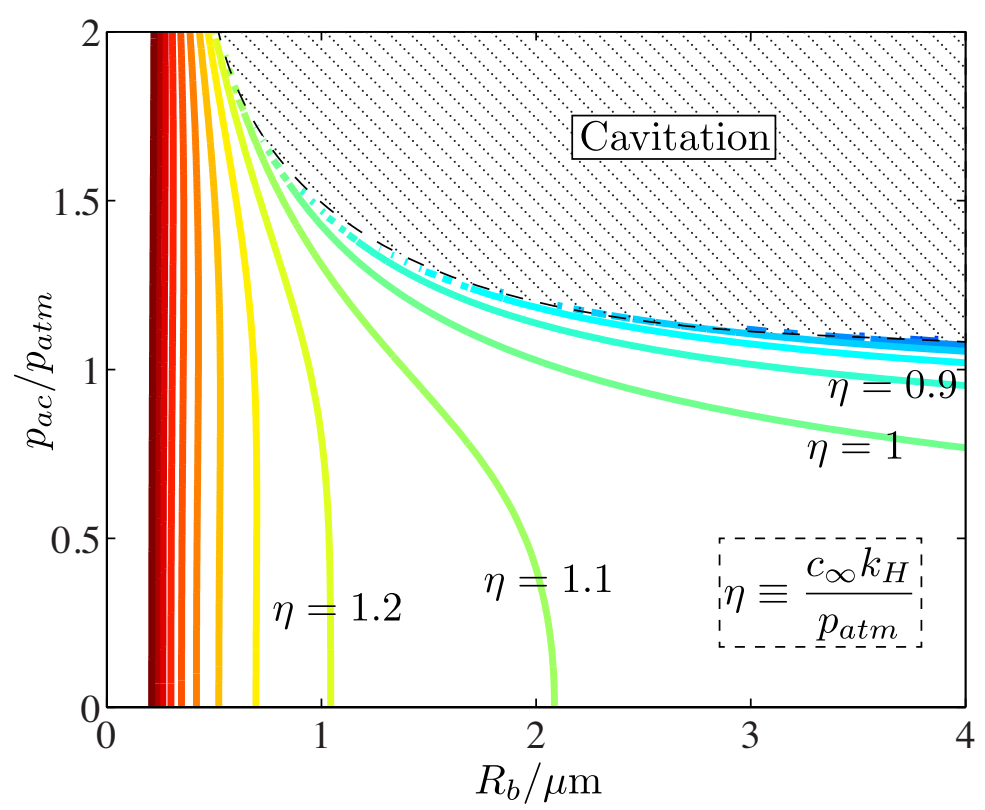

Figure 5.7: Isodensity contours of $\left\langle\hat{p}_{b u b}\right\rangle_{\tau}$ for nanobubbles with equilibrium $\left(p_{\text {liq }}=p_{\text {atm }}\right)$ contact angle $\theta_{0}=20^{\circ}$ of various sizes $R_{b}$ and at different acoustic driving pressures $p_{a c}$ in a liquid with surface tension $\gamma=0.03 \mathrm{~J} / \mathrm{m}^{2}$. Comparing the isolines of $\left\langle\hat{p}_{b u b}\right\rangle_{\tau}$ against the equivalent pressure of the far-field concentration $c_{\infty} k_{H}$ then results in a stability phase diagram. Here, the region above a specific line denotes the area of growth by rectified diffusion at a specific initial dissolved gas concentration (characterized by $\eta=c_{\infty} k_{H} / p_{a t m}$ ). The striped region indicates the parameters at which, at some time during one acoustic cycle, the surrounding pressure $p_{\text {liq }}$ becomes so low that the bubble experiences unbounded growth (cavitation). This region is therefore the upper limit for the acoustic pressure amplitude $p_{a c}$. Therefore, for given bubble size $R_{b}$, the iso-line corresponding to the far-field gas concentration $c_{\infty}$ indicates the minimal required driving amplitude $p_{a c}$. For a nanobubble for which $R_{b}=1 \mu \mathrm{m}$ at mild supersaturation, say: $\eta=1.2$, we therefore recover growth when $p_{a t m}<p_{a c}<1.5 p_{a t m}$. 
the phase diagram when $\gamma>0$. For small bubbles $R_{b} \rightarrow 0$ the required supersaturation at finite acoustic driving pressures diverges. This can be explained by the extremely high curvatures of the liquid-gas interface for such nanobubbles.

\section{Dynamic behaviour}

The analyses above were performed at constant $N$, and provide great insight in the required driving pressures and supersaturation to achieve bubble growth through rectified diffusion. In reality, $p_{a c}$ is held fixed while $N$ varies as bubbles grow or shrink. The corresponding phase diagram is shown in Fig. 5.8(a). Here, we see that at an acoustic driving pressure of $p_{a c}=1.5 p_{a t m}$, various stable and unstable equilibria points exist at varying saturation levels $\eta$ and bubble sizes $R_{b}$. Suppose, for example, a very wide bubble with $R_{b}=800 \mathrm{~nm}$ in a solution that is only slightly supersaturated: $\eta=1.1$. In Fig. 5.8(a) several starting contact angles $\theta_{0}(0)$ are selected. When the bubble is flat enough $\left(\theta_{0} \lesssim 27^{\circ}\right)$, the bubble grows or shrinks towards a stable equilibrium at $\sim 6^{\circ}$. Bubbles for which $\theta_{0} \gtrsim 27^{\circ}$ experience unbounded growth until they reach the cavitation regime. In Fig. $5.8(\mathrm{~b})$ the numerically calculated result of Eqs. (5.37) and (5.38) are shown for these starting point, confirming the behaviour expected from the phase diagram. Using this phase diagram, therefore, we can predict the asymptotic behaviour in time of any surface (nano)bubble. The nondimensional time $\mathcal{T}=6 \cdot 10^{4}$ in Fig. 5.8(b) corresponds to real-time $t=38.4 \mathrm{~s}$.

We can now compare the full numerics from section 5.4 , specifically figure 5.4 where $\eta=1$, with the phase diagram in figure 5.8. We see that the results agree qualitatively. The smaller of the two bubbles $\left(R_{b}=100 \mathrm{~nm}\right.$, figure 5.4(a)) indeed shrinks as also predicted in the phase diagram. The larger of the two bubbles $\left(R_{b}=1 \mu \mathrm{m}\right.$, figure $\left.5.4(\mathrm{~b})\right)$ was found to grow. Indeed, we find in the phase diagram that for $\eta=1$ at $\theta=10^{\circ}$ we should expect unbounded growth until the bubble cavitates. This cavitation regime is not reached in the full numerics because of the limited time-range accessible using this method.

\section{Comparison with experiments}

In this section, we compare the results discussed above with the experimental results from section 5.2. We note that, due to approximations (e.g. the $1 \mathrm{D}$ flat bubble-approximation) and unknown parameters in the experiment (e.g. 


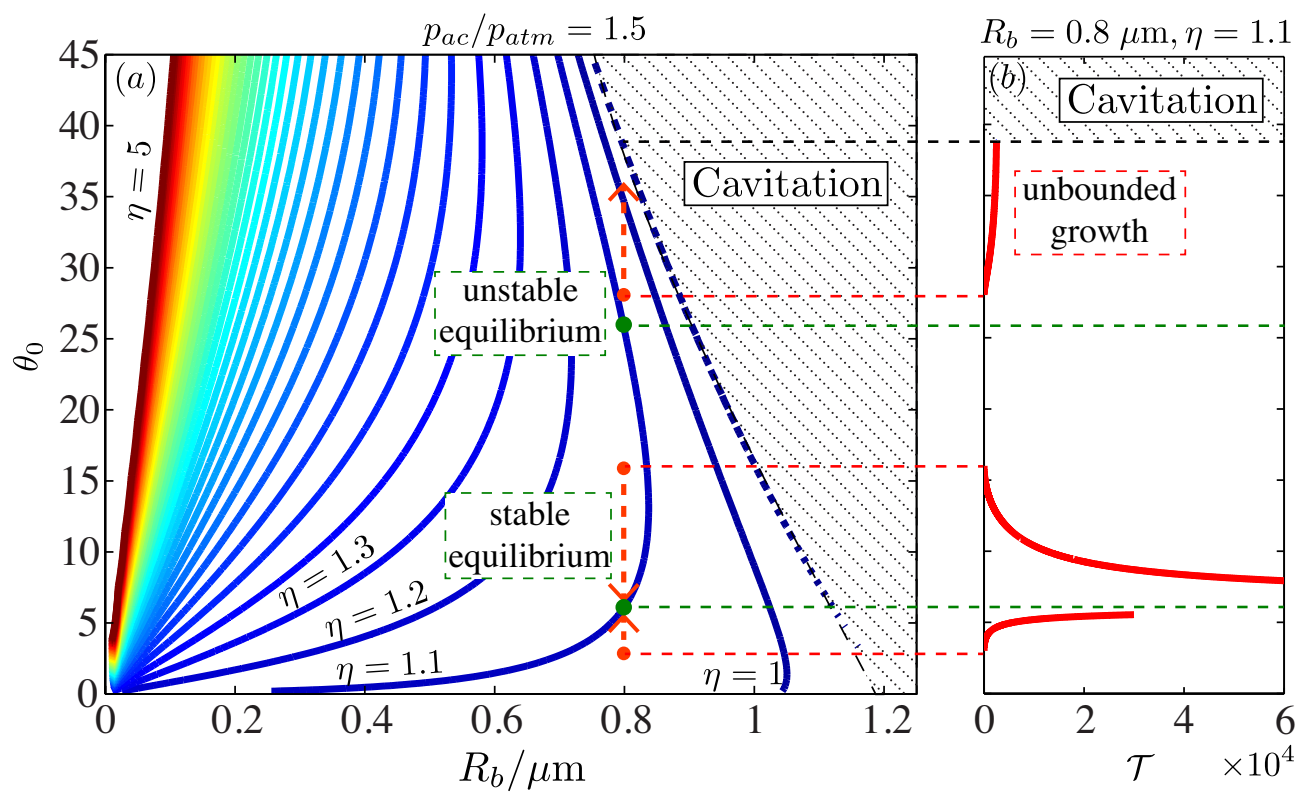

Figure 5.8: Phase diagram for bubble growth/shrinkage at fixed driving pressure $p_{a c} / p_{a t m}=1.5$. Each line indicates the boundary between growth (right) and shrinkage (left) at given saturation level $\eta$ of nanobubbles of different lateral sizes $R_{b}$ and (equilibrium) contact angles $\theta_{0}$. Note that $\theta_{0}$ is a monotonically increasing function with $N$, i.e. the contact angle of growing bubbles always increases. The red arrows indicate the behaviour in time for a bubble with a lateral size of $R_{b}=0.8 \mu \mathrm{m}$ in a slightly supersaturated solution $(\eta=1.1)$. For $\theta_{0} \lesssim 6^{\circ}$ the bubble grows towards an equilibrium size. For $6^{\circ} \lesssim \theta_{0} \lesssim 27^{\circ}$ the bubble shrinks towards the same equilibrium size. If the bubble is larger, $\theta_{0} \gtrsim 27^{\circ}$ the bubble experiences unbounded growth and ends up in the cavitation regime. If the same bubble were placed in a solution where $\eta=1.2$ the bubble would always experience unbounded growth whereas if it were placed in a solution where $\eta=1$ the bubble would shrink until it has disappeared. 

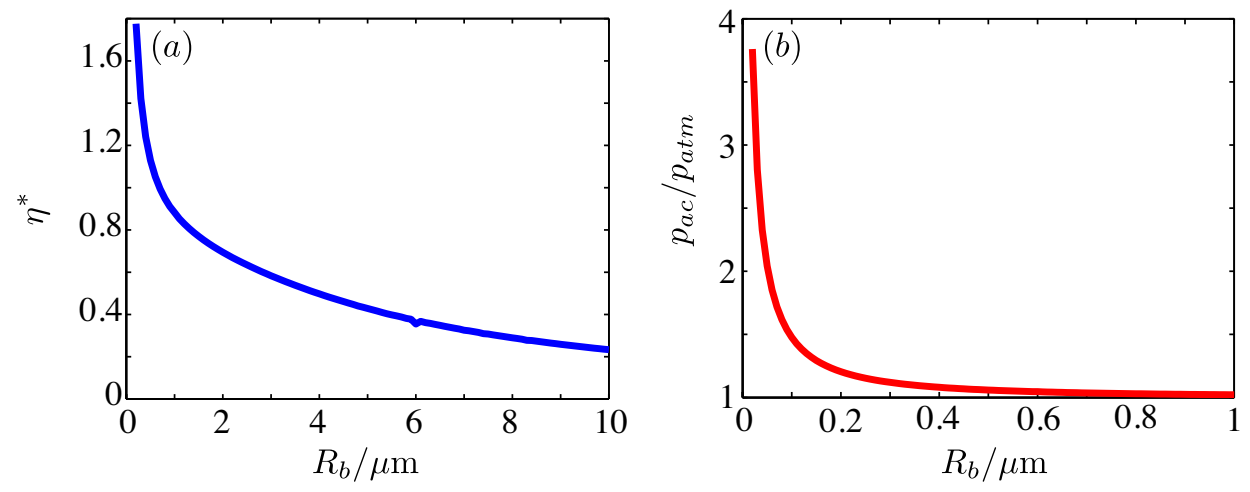

Figure 5.9: Threshold values at which growth by rectified diffusion can occur. (a) At given footprint radius $R_{b}$, bubbles can only grow when $\eta>\eta^{*}$. Smaller bubbles require higher gas concentrations. (b) Similarly, smaller bubbles also require higher acoustic driving pressures.

supersaturation level and distribution, acoustic pressure) we cannot expect quantitative agreement. Therefore, we discuss the quantitative similarities between the model and the experiments.

First, we check whether growth by rectified diffusion can realistically be expected in the experimental situation from sect 5.2. For example: What amount of supersaturation is required to be able to induce growth by rectified diffusion for nanobubbles of a certain size? We evaluate $\left\langle\hat{p}_{b u b}\right\rangle_{\tau}$ at the cavitation threshold (dashed line in Fig. 5.7), which indicates the leftside boundary in the phase-diagram, and gives the minimum value of $\eta$, denoted $\eta^{*}$, for which growth by rectified diffusion can occur. The result is shown in Fig. 5.9(a). The dependence of critical supersaturation $\eta^{*}$, where we see that, when the acoustic driving is maximal (tuned just below the strength where cavitation would occur) larger bubbles $\left(R_{b} \gtrsim 1.5 \mu \mathrm{m}\right)$ do not require a supersaturated liquid. For surface nanobubbles with $R_{b} \approx 100 \mathrm{~nm}$, a supersaturation of approximately $60 \%$ is required at an acoustic driving pressure of $p_{a c} \approx 1.5 \mathrm{~atm}$. Although the exact amount of supersaturation in the experiments is unknown, a value of $60 \%$ is not unrealistic at all as the air-equilibrated solvent was heated prior to the experiments. Furthermore, the ethanol-water exchange process used to generate nanobubbles also leads to supersaturation. We can also check whether the required driving pressure $p_{a c}$ is close to the approximate $p_{a c}=1.5 p_{a t m}$ in the experiments. The required driving pressure to sustain growth at the saturation level $\eta^{*}$ is 
shown in Fig. 5.9(b). We see that in order to sustain growth for the smaller nanobubbles, an increasingly higher driving pressure is required. This is to be expected because the Laplace pressure becomes stronger as the bubble is smaller. For the experimental value, we see that a bubble with $R_{b} \approx 80 \mathrm{~nm}$ can be sustained. Although this is on the large side for surface nanobubbles, the order of magnitude is correct which is all that can be expected given the approximations and unknowns.

\section{$5 \cdot 7$ Conclusion}

We have theoretically shown and quantified that the nanobubble growth found in the experiments discussed in section 5.2 and in ref. [1] can indeed be caused by rectified diffusion. At the given experimental conditions, nanobubbles with footprint sizes of order $10-100 \mathrm{~nm}$ indeed grow, given that the acoustic driving is strong enough. We have provided a detailed phase diagram that shows that bubbles can either shrink, experience unbounded growth or grow/shrink towards an equilibrium radius, depending on the driving parameters. Future work should be focused on a geometrically more accurate description of the nanobubbles: Particularly the effect of neighboring nanobubbles. In the case of driving, we have seen that complex concentration profiles develop around the nanobubbles (cf. Fig. 5.5) which may affect nearby nanobubbles. Additionally, it was found that some nanobubbles move across the substrate during the application of ultrasound. This is a particularly interesting effect, from which we can learn whether, and how strongly, nanobubbles are pinned. A more elaborate discussion on this can be found in chapter 11 of this thesis.

\section{References}

[1] A. Brotchie and X. H. Zhang, Response of interfacial nanobubbles to ultrasound irradiation, Soft Matter 7, 265 (2011).

[2] P. Ball, How to keep dry in water, Nature 423, 25 (2003).

[3] P. G. de Gennes, On fluid/wall slippage, Langmuir 18, 3413 (2002).

[4] V. S. J. Craig, Very small bubbles at surfaces - the nanobubble puzzle, Soft Matter 7, 40 (2011). 
[5] M. A. Hampton and A. V. Nguyen, Nanobubbles and the nanobubble bridging capillary force, Adv. Coll. Int. Sci. 154, 30 (2010).

[6] J. R. T. Seddon and D. Lohse, Nanobubbles and micropancakes: Gaseous domains on immersed substrates, J. Phys. Cond. Mat. 23, 133001 (2011).

[7] J. L. Parker, P. M. Claesson, and P. Attard, Bubbles, cavities, and the longranged attraction between hydrophobic surfaces, J. Phys. Chem. 98, 8468 (1994).

[8] X. H. Zhang, A. Khan, and W. A. Ducker, A nanoscale gas state, Phys. Rev. Lett. 98, 136101 (2007).

[9] X. H. Zhang, A. Quinn, and W. A. Ducker, Nanobubbles at the interface between water and a hydrophobic solid, Langmuir 24, 4756 (2008).

[10] X. H. Zhang, Quartz crystal microbalance study of the interfacial nanobubbles, Phys. Chem. Chem. Phys. 10, 6842 (2008).

[11] S. Karpitschka, E. Dietrich, J. R. T. Seddon, H. J. W. Zandvliet, D. Lohse, and H. Riegler, Nonintrusive Optical Visualization of Surface Nanobubbles, Phys. Rev. Lett. 109, 066102 (2012).

[12] C. U. Chan and C.-D. Ohl, Total-Internal-Reflection-Fluorescence Microscopy for the Study of Nanobubble Dynamics, Phys. Rev. Lett. 109, 174501 (2012).

[13] R. Steitz, T. Gutberlet, T. Hauss, B. Klösgen, R. Krastev, S. Schemmel, A. C. Simonsen, and G. H. Findenegg, Nanobubbles and their precursor layer at the interface of water against a hydrophobic substrate, Langmuir 19, 2409 (2003).

[14] D. A. Doshi, E. B. Watkins, J. N. Israelachvili, and J. Majewski, Reduced water density at hydrophobic surfaces: Effect of dissolved gases, PNAS 102, 9458 (2005).

[15] M. Mezger, H. Reichert, S. Schöder, J. Okasinski, H. Schröder, H. Dosch, D. Palms, J. Ralston, and V. Honkimäki, High-resolution in situ $x$-ray study of the hydrophobic gap at the water-octadecyl-trichlorosilane interface, Proc. Nat. Acad. Sci. 103, 18401 (2006).

[16] W. A. Ducker, Contact Angle and Stability of Interfacial Nanobubbles, Langmuir 25, 8907 (2009). 
[17] S. Das, J. H. Snoeijer, and D. Lohse, Effect of impurities in description of surface nanobubbles, Phys. Rev. E 82, 056310 (2010).

[18] M. P. Brenner and D. Lohse, Dynamic equilibrium mechanism for surface nanobubble stabilization, Phys. Rev. Lett. 101, 214505 (2008).

[19] J. R. T. Seddon, H. J. W. Zandvliet, and D. Lohse, Knudsen gas provides nanobubble stability, Phys. Rev. Lett. 107, 116101 (2011).

[20] E. Dietrich, H. J. W. Zandvliet, D. Lohse, and J. R. T. Seddon, Particle tracking around surface nanobubbles, J. Phys.-Condens. Matter 25, 184009 (2013).

[21] J. H. Weijs, J. H. Snoeijer, and D. Lohse, Formation of Surface Nanobubbles and the Universality of Their Contact Angles: A Molecular Dynamics Approach, Phys. Rev. Lett. 108, 104501 (2012).

[22] X. Zhang, D. Y. C. Chan, D. Wang, and N. Maeda, Stability of Interfacial Nanobubbles, Langmuir 29, 1017 (2013).

[23] Y. Liu and X. Zhang, Nanobubble stability induced by contact line pinning, J. Chem. Phys. 138, 014706 (2013).

[24] R. P. Berkelaar, H. J. Zandvliet, and D. Lohse, Covering surface nanobubbles with a $\mathrm{NaCl}$ nano-blanket, Langmuir Submitted, (2013).

[25] A. Eller and H. G. Flynn, Rectified Diffusion During Nonlinear Pulsations of Cavitation Bubbles, J. Acoust. Soc. Am. 37, 493 (1964).

[26] C. E. Brennen, Cavitation and Bubble Dynamics (Oxford University Press, Oxford, 1995).

[27] M. M. Fyrillas and A. J. Szeri, Dissolution or growth of soluble spherical oscillating bubbles, J. Fluid Mech. 277, 381 (1994).

[28] S. Hilgenfeldt, D. Lohse, and M. P. Brenner, Phase diagrams for sonoluminescing bubbles, Phys. Fluids 8, 2808 (1996).

[29] M. P. Brenner, S. Hilgenfeldt, and D. Lohse, Single bubble sonoluminescence, Rev. Mod. Phys. 74, 425 (2002).

[30] B. M. Borkent, S. M. Dammer, H. Schönherr, G. J. Vancso, and D. Lohse, Superstability of surface nanobubbles, Phys. Rev. Lett. 98, 204502 (2007). 
[31] P. Attard, Nanobubbles and the hydrophobic attraction, Adv. Colloid Int. Sci. 104, 75 (2003).

[32] X. Zhang, H. Lhuissier, O. R. Enriquez, C. Sun, and D. Lohse, Deactivation of Microbubble Nucleation Sites by Alcohol-Water Exchange, Langmuir (in press), (2013).

[33] E. Hinch, Pertubation methods (Cambridge University Press, New York, 1995).

[34] A. Prosperetti, Advanced Mathematics for Applications (Cambridge University Press, New York, 2011). 


\section{6 Why is surface tension a force parallel to the interface? *}

A paperclip can float on water. Drops of mercury refuse to spread on a surface. These capillary phenomena are macroscopic manifestations of molecular interactions, and can be explained in terms of surface tension. For students, the concept of surface tension is quite challenging since the microscopic intuition is often in conflict with the common macroscopic interpretations. In this chapter we address a number of conceptual questions that are often encountered when teaching capillarity. By answering these questions we provide a perspective that reconciles the macroscopic viewpoints, from thermodynamics or fluid mechanics, and the microscopic perspective from statistical physics.

\subsection{Basic concepts and problems}

Capillarity is one of the most interesting subjects to teach in condensed matter physics, as its detailed understanding involves three otherwise separated domains: macroscopic thermodynamics [1-3], fluid mechanics and statistical physics [4]. The microscopic origin of surface tension lies in the intermolec-

${ }^{*}$ Published as: A. Marchand, J. H. Weijs, J. H. Snoeijer, and B. Andreotti, "Why is surface tension a force parallel to the interface?", Am. J. Phys. 79, 9992011 
ular interactions and thermal effects $[5,6]$, while macroscopically it can be seen as a force acting along the interface or an energy per unit surface. In the present article we discuss the link between these three aspects of capillarity, on the basis of simple academic examples. We first discuss the standard problems faced by students - and many researchers - in the understanding of surface tension. We will see that the difficulty of understanding surface tension forces is often caused by the improper or incomplete definition of a system on which the forces act. We bring up four basic questions, such as the one raised in the title, which are answered in the rest of the article. Contrary to many textbooks on the subject, this provides a picture that reconciles the microscopic, thermodynamic and mechanical aspects of capillarity.

\subsubsection{The interface}

\section{Thermodynamic point of view}

Following the pioneering work of Gibbs [7], we introduce surface tension as the excess free energy due to the presence of an interface between bulk phases. Let us consider a molecule in the vicinity of an interface, for example near a liquid-vapor interface. The environment of this molecule is manifestly different from the molecules in the bulk. This is usually represented schematically by drawing the attractive bonds around molecules, as shown in Fig. 6.1. One clearly sees from this picture that approximately half of the bonds are missing for a molecule at the interface, leading to an increase of the free energy. One thus defines the surface tension from the free energy $F$ per unit area:

$$
\gamma_{\mathrm{LV}}=\left(\frac{\partial F}{\partial A}\right)_{T, V, n},
$$

for a system of volume $V$ containing $n$ molecules at temperature $T$. Hence, $\gamma_{\mathrm{LV}}$ is the energy needed to increase the interfacial area by one unit. Its dimension is $\left[\gamma_{\mathrm{LV}}\right]=M \cdot T^{-2}$ (mass per time squared) and it is usually expressed in $\mathrm{N} / \mathrm{m}$ (force per unit length) or $\mathrm{J} / \mathrm{m}^{2}$ (energy per unit area).

It is instructive to estimate the magnitude of surface tension, which must be of the order of the bond energy $\epsilon$ divided by the cross section area $\sigma^{2}$ of a molecule $-\sigma$ is a fraction of nanometer. For oils, interaction through van der Waals interactions leads to $\epsilon \sim k_{B} T \simeq \frac{1}{40} \mathrm{eV}$ and thus $\gamma_{\mathrm{LV}} \sim 0.02 \mathrm{~N} / \mathrm{m}$. For water, hydrogen bonds lead to a higher value $\gamma_{\mathrm{LV}} \sim 0.072 \mathrm{~N} / \mathrm{m}$. For mercury, the high energy bonds $(\epsilon \sim 1 \mathrm{eV})$ lead to a high surface tension $\gamma_{\mathrm{LV}} \sim 0.5 \mathrm{~N} / \mathrm{m}$. 


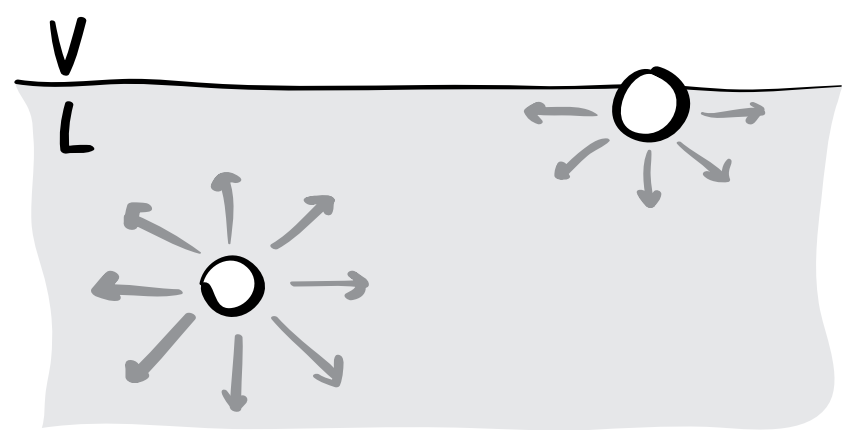

Figure 6.1: Sketch showing the 'missing' intermolecular bonds close to the liquidvapor interface. This gives rise to an increase in free energy per unit area, i.e. the surface tension.

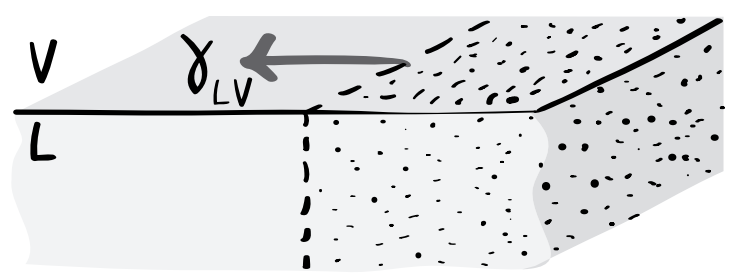

Figure 6.2: Sketch showing surface tension as a force per unit length exerted by one subsystem on the other. The system on which the forces act is the dotted region. The force is parallel to the interface and perpendicular to the dividing line.

\section{Mechanical point of view}

In fluid mechanics, the surface tension is not defined in terms of a surface energy but rather as a force per unit length. In the bulk of a fluid at rest, two sub-parts of a fluid exert a repulsive interaction on one another, which is called the pressure. If the surface separating these two subsystems crosses the liquid-vapor interface, an additional force needs to be taken into account: surface tension. As shown in Fig. 6.2, the surface tension is a force tangent to the surface and normal to the contour separating the two subsystems. The total force is proportional to the width of the contour, which we will call $W$ throughout the paper. Contrarily to pressure, surface tension is an attractive force.

The link between mechanics and thermodynamics is provided by the vir- 
tual work principle. If one moves a contour of width $W$ by a length $d l$, the area of the interface of the subsystem considered increases by Wdl. Consequently, the free energy is increased by $\gamma_{\mathrm{LV}} W d l$. The free energy should equal the work done by the surface tension force, which means that this force must be parallel to the interface, normal to the contour and have a magnitude $\gamma_{\mathrm{LV}} W$. Per unit length, the tension force is thus $\gamma_{\mathrm{LV}}$.

On the other hand, the link between mechanics and statistical physics is much less obvious for students. One clearly sees in Fig. 6.1 that the molecule at the interface is submitted to a net force (which would be represented by the sum of the vectors) along the direction perpendicular to the interface. However, we just argued from the mechanical point of view, that the force is parallel to the interface. This leads to the first key question of this article:

- Q1: Why is surface tension a force parallel to the interface while it is so obvious that it must be perpendicular to it?

\subsubsection{The contact line}

\section{Thermodynamic point of view}

A standard experimental method for determining the liquid-vapor surface tension is to measure the force required to pull a metallic plate (usually made of platinum) out of a liquid bath. This force is related to the liquidvapor surface tension $\gamma_{\mathrm{LV}}$, as is usually explained by the diagram of Fig. 6.3a. Imagine that the plate is moved vertically by a distance $d l$. The area of the liquid-vapor interface does not change by this motion, so the corresponding interfacial energy is unaffected. However, the motion does lead to a decrease of the immersed solid-liquid interface area, by $W d l$, while the solid-vapor interface increases by the same amount. In other words, part of the wetted surface is exchanged for dry surface. This leads to a variation of the free energy $d F=\left(\gamma_{\mathrm{SV}}-\gamma_{\mathrm{SL}}\right) W d l$, where $\gamma_{\mathrm{SV}}$ and $\gamma_{\mathrm{SL}}$ are the solid-vapor and solid-liquid surface tensions respectively. This energy is provided by the work done by the experimentalist, due to the force required to displace the plate by $d l$. Hence, this force must be equal to $\left(\gamma_{S V}-\gamma_{S L}\right) W$.

To relate this force to the value of the liquid-vapor surface tension $\gamma_{\mathrm{LV}}$, one has to invoke Young's law for the contact angle $\theta$. When the three interfaces (between solid, liquid and vapor) join at the contact line, the liquid makes contact with the substrate at an angle $\theta$ given by[8]:

$$
\gamma_{\mathrm{LV}} \cos \theta=\gamma_{\mathrm{SV}}-\gamma_{\mathrm{SL}} \text {. }
$$



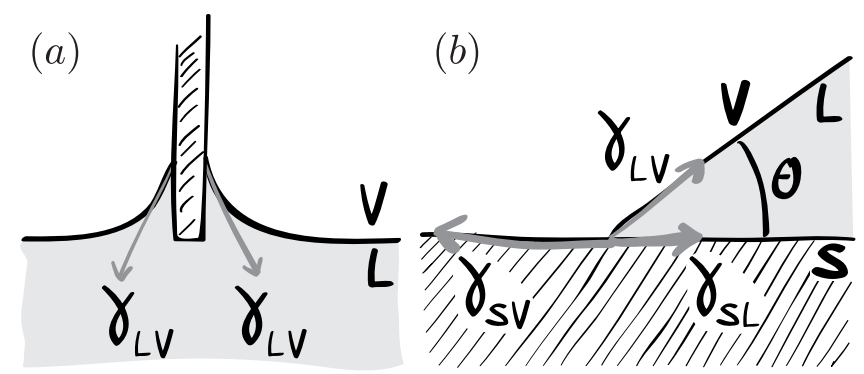

Figure 6.3: (a) Experimental method to determine the liquid-vapor surface tension. The force per unit length needed to pull a plate from a bath of liquid is equal to $\gamma_{\mathrm{LV}} \cos \theta$, where $\theta$ is the equilibrium contact angle. (b) A classical way to interpret Young's law as a force balance of surface tensions. Question 2: Why is there no force balance in the normal direction? Question 3: Why do we draw a single surface tension force in the left panel $\left(\gamma_{\mathrm{LV}}\right)$ while there are three in the right panel $\left(\gamma_{\mathrm{LV}}, \gamma_{\mathrm{SV}}, \gamma_{\mathrm{SL}}\right)$ ?

With this, the force exerted on the plate can be expressed as $W \gamma_{\mathrm{LV}} \cos \theta$, and can thus be used to design a tensiometer.

\section{Mechanical point of view}

From the mechanical point of view, we can interpret this force as the surface tension acting parallel to the liquid-vapor interface. By symmetry, the total force exerted on the solid is vertical (the horizontal components add up to zero). Projecting the surface tension force on the vertical direction and multiplying by the length $W$ of the contact line, one indeed gets $W \gamma_{\mathrm{LV}} \cos \theta$.

By a similar argument one usually interprets Young's law for the contact angle as the balance of forces at the contact line (Fig. 6.3b). By projection along the direction parallel to the solid substrate, one obtains $\gamma_{\mathrm{SL}}+$ $\gamma_{\mathrm{LV}} \cos \theta=\gamma_{\mathrm{SV}}$, which is the same as equation (6.2). This force interpretation is a common source of confusion for students:

- Q2: From Fig. 6.2b, there seems to be an unbalanced force component in the vertical direction $\gamma_{\mathrm{LV}} \sin \theta$. What force is missing to achieve equilibrium?

- Q3: Why does one draw a single force acting on the contact line in the case of the plate (Fig. 6.3a), while for Young's law we need to balance all three forces (Fig. 6.3b)?

Actually, when measuring a surface tension using the plate technique, one often uses a platinum plate to be sure that the liquid completely wets the solid. In that case, however, $\gamma_{\mathrm{SV}}-\gamma_{\mathrm{SL}}>\gamma_{\mathrm{LV}}$ and Young's law does not 
apply! Then, the thermodynamic and mechanical approaches give conflicting answers:

- Q4: In the situation of complete wetting, is the force on the plate given by $\gamma_{\mathrm{LV}}$ or by $\gamma_{\mathrm{SV}}-\gamma_{\mathrm{SL}}$ ?

\subsubsection{Answers}

Before addressing these points in detail, we start with a short overview of the answers to the questions raised above. We emphasize that the thermodynamic result (i.e. from the virtual work principle) always gives the correct total force. If one wants to know the local force distribution, which cannot be extracted from thermodynamics, it is imperative that the system on which the forces act is properly defined. Confusion regarding the forces is often caused by an improper or incomplete definition of such a system.

- A1: The schematic of Fig. 6.1 represents only the attractive intermolecular forces. The real force balance requires both repulsive and attractive interactions between liquid molecules.

To answer the questions related to the contact line it is crucial to specify the system of molecules on which the forces are acting:

- A2: In Young's law, the system on which the forces act is a corner of liquid bounded by the contact line. $\gamma_{\mathrm{LV}}$ is indeed the force exerted on this system inside the liquid-vapor interface, but the forces exerted by the solid on the corner are incomplete in Fig. 6.3b. An extra vertical force on the liquid, caused by the attraction of the solid, exactly balances the upward force $\gamma \sin \theta$.

- A3: To obtain the force on the plate, the system to consider is the solid plate. In this case, the force exerted by the liquid on the solid is in fact equal to $\gamma_{\mathrm{LV}} \cos \theta$ per unit length.

- A4: The correct vertical force on the plate is $W \gamma_{\mathrm{LV}} \cos \theta$. In the case of complete wetting $(\theta=0)$, the virtual work principle can be applied, but only when taking into account the prewetting film.

\subsection{Microscopic interpretation of capillarity}

\subsubsection{The liquid state}

To address the origin of capillarity, we first have to understand how a liquid phase and a vapor phase can coexist. This goes back to the work of van der Waals who derived an equation of state for fluids that can account for a 


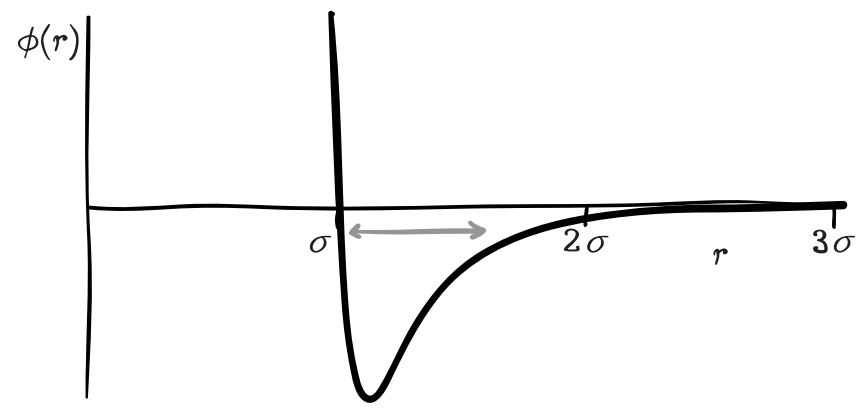

Figure 6.4: Lennard-Jones intermolecular potential $\phi$. The interaction is strongly repulsive for intermolecular distances $r<\sigma$, where $\sigma$ reflects the 'hard-core' of the molecules. At large distances the molecules are attracted to one another. The gray arrow points to the presence of thermal fluctuations, which, in a liquid, lead to substantial variations of the intermolecular distance.

liquid-gas phase transition[5, 9]:

$$
P=\frac{k T}{v-b}-\frac{a}{v^{2}} .
$$

Here $P$ is the pressure, $v$ the volume per molecule, and $T$ the temperature. This equation of state corrects the ideal gas law to incorporate the effect of intermolecular forces. The constant $b$ introduces repulsion between molecules as an excluded volume effect: the pressure diverges when the total volume per molecule reaches a minimal size $b$. In this limit the molecules are densely packed and constitute a liquid phase. In this phase, the volume per molecule no longer depends on pressure, which means that the liquid phase is incompressible. Ultimately, this effect comes from the repulsion of the electron clouds of the molecules, due to Pauli exclusion principle in quantum mechanics. The constant $a$ represents the long-range attraction between molecules which finds its origin in the dipole-dipole interaction (van der Waals attraction).

Van der Waals's equation of state (6.3) explains how a low density gas phase can coexist with a high density liquid phase. This coexistence requires the pressures to be identical on both sides of the interface, despite the striking difference in density. In a gas, where $v=v_{g}$ is large, most of the energy is of kinetic origin $\left(a / v_{g}^{2} \ll P\right)$ : the pressure is $P \simeq k T / v_{g}$. In the liquid phase the volume per unit molecule is almost in the incompressible limit: $v=v_{l} \approx b$. This strong repulsive effect $\left(k T /\left(v_{l}-b\right) \gg P\right)$, however, is coun- 
terbalanced by the presence of attractions $\left(a / v_{l}^{2} \gg P\right)$ so that, for the same temperature, the pressure in the liquid phase can be in equilibrium with the pressure in the gas phase. This gives rise to a stable liquid-vapor interface.

How can a liquid at the same time be repulsive and attractive? A single pair of atoms can of course only attract or repel each other, depending on the distance separating their two nuclei. This interaction is shown schematically in Fig. 6.4. The steep potential reflects the short-range repulsion, while the negative tail of the potential represents the long-range attraction. This means that the balance of attractive and repulsive interactions in (6.3) only has a statistical meaning: some particles are in an attractive state while others are in a repulsive state. This property of 'simultaneous' repulsion and attraction is what makes a liquid very different from a solid: in an elastic solid, molecules in the same region are either all compressed (if the solid is submitted to an external compression), or all attracted to each other (if the solid is submitted to an external tension).

The difference between a solid and a liquid can be traced back to the importance of thermal fluctuations, i.e. the kinetic energy of the molecules. In the solid phase, these fluctuations are relatively small with respect to the potential energy, i.e. $k_{B} T \ll \epsilon$, where $\epsilon$ is the energy scale for the intermolecular forces. As a consequence the system only explores a small region of the potential. Hence the solid is either in the compressed or in the tensile state. On the other hand, the liquid phase is characterized by large fluctuations, for which $k_{B} T \sim \epsilon$. A broad range of the potential is therefore sampled by molecules in the same region of space (Fig. 6.4). Finally, the case $k_{B} T \gg \epsilon$ corresponds to a gas phase of weakly interacting particles that is dominated by kinetic energy.

\subsubsection{The liquid-vapor interface: Question 1}

\section{The force of surface tension}

Let us now consider the liquid-vapor interface in more detail. Figure 6.5a shows a snapshot of the interface obtained in Molecular Dynamics simulations of molecules that interact through a Lennard-Jones potential [10, 11]. The corresponding time-averaged density profile is plotted in Fig. 6.5b. The transition from the high density liquid to the low density gas takes place in a very narrow region that is a few molecules wide. In order to determine the capillary forces we need to divide the system along the direction normal to the interface into two subsystems (see Fig. 6.5a). One considers the 


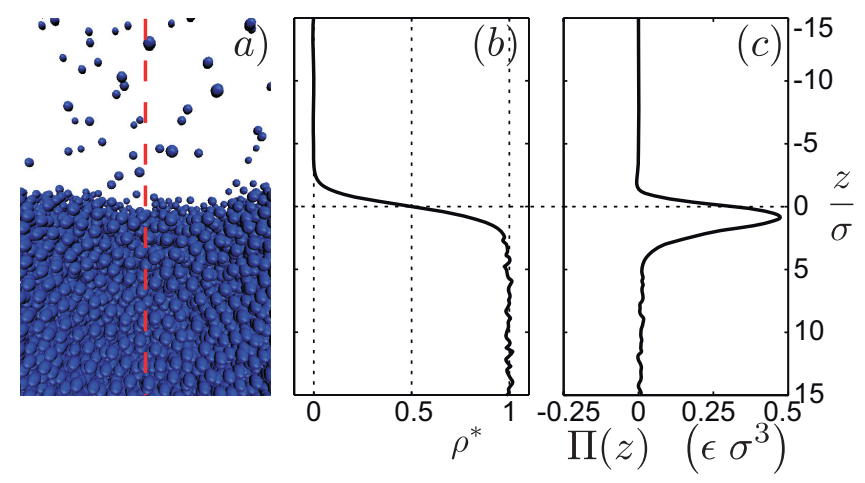

Figure 6.5: The liquid-vapor interface. The vertical axis is in units of the molecular scale $\sigma$. (a) Snapshot of Lennard-Jones simulation of a liquid-vapor interface. (b) Time-averaged normalized density profile $\rho^{*}(z)$ across the interface. (c) Tangential force per unit area exerted by the left part on the right part of the system. Technically speaking, the plot shows the difference $\Pi=p_{N N}-p_{T T}$ between the normal and tangential components of the stress tensor.

force per unit area, called the stress, exerted by the left subsystem on the right subsystem as a function of the vertical position $z$. This stress can be decomposed into two contributions: the pressure $P$, which we recall to be the same in the vapor and the liquid bulk, plus an extra stress $\Pi(z)$ acting along the direction parallel to the interface, plotted in Fig. 6.5c. The profile of this stress anisotropy shows that there is a force localized at the interface, acting in the direction parallel to the interface. This force spreads over a few molecular scales, which is also the typical thickness of the density jump across the interface. The integrated contribution of this force is indeed equal to $\gamma_{\mathrm{LV}}$ per unit length, the surface tension. This shows that surface tension really is a mechanical force.

Having seen that in our simulations there is a parallel force localized at the interface, let us turn to Question 1. Why is the tension force parallel and not normal to the interface? First, we note that Fig. 6.1 only depicts the attraction between molecules. A more complete picture also incorporates the repulsive forces in the internal pressure, as sketched as dashed arrows in Fig. 6.6. Away from the surface there is perfect force balance due to the symmetry around the molecule. Near the interface, however, the up-down symmetry is broken. To restore the force balance in the vertical direction, the upward repulsive arrow (dashed) has to balance with the downward at- 


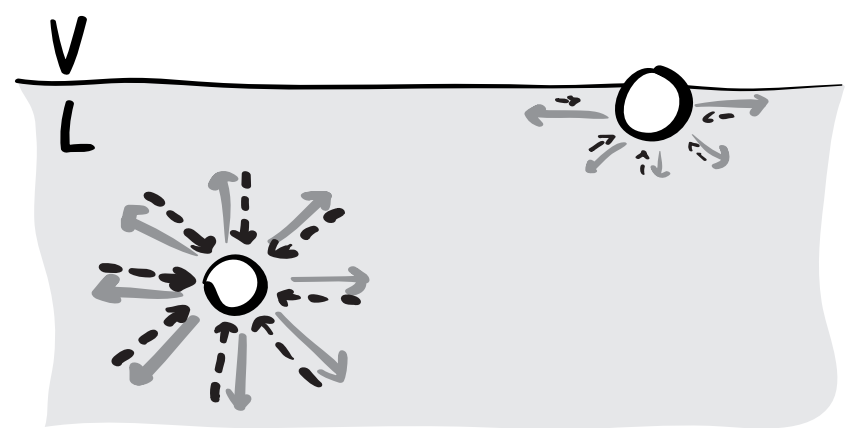

Figure 6.6: Sketch showing repulsive (dashed black arrows) and attractive (gray arrows) forces in the bulk and at the surface.

tractive arrow (solid). In the direction parallel to the interface the symmetry is still intact, thus automatically ensuring a force balance parallel to the interface. This means that along the direction parallel to the interface, there is no reason why the attractive forces should have the same magnitude as the repulsive forces. As described above, we find that in practice, the attractive forces are stronger, which indeed give rise to a positive surface tension force.

\section{Separate roles of attraction and repulsion}

However, we still need to explain why these intermolecular forces give rise to such a strong tension along the surface. This question was previously addressed by Berry[6], who noted the separate roles of attraction and repulsion. The key observation is that, to a good approximation, the repulsive contribution to the pressure is isotropic while attraction is strongly anisotropic. This is because the repulsion is very short ranged due to the hard core of the molecules, and can therefore be thought of as a "contact force". As such, repulsion is not very sensitive to the changes in molecular structure near the interface and is equally strong in all directions. By contrast, the long-range nature of the attractive forces make them very susceptible for the molecular structure. This is the origin of the observed pressure anisotropy near the interface that generates the surface tension force.

To see how this works out in detail it is useful to divide the liquid into two subsystems using control surfaces parallel to the liquid-vapor interface, as shown in Fig. 6.7a. The force exerted on the dotted subsystem by the rest of the liquid results from the superposition of attractive (vertical gray 
arrows) and repulsive (dashed black arrows) interactions (Fig. 6.7a). As the subsystem is at equilibrium, these attractive and repulsive components must balance each other. The magnitude of the attractive force increases with the size of attracting region - this is because the density increases as one moves from the vapor towards the liquid phase. The magnitude of the attraction saturates to the bulk value when the control surface is a few molecular sizes from the interface. We then divide the liquid into two subsystems using a control surface perpendicular to the liquid vapor interface (Fig. 6.7b). One can now use that the repulsive short-range forces are isotropic. This means that the magnitude of repulsion (dashed black arrows) exerted by the left side on the subsystem (dotted region) increases with depth in a way analogous to that in Fig. 6.7a. By contrast, the strength of attraction has a much weaker dependence on depth - for the sake of simplicity we draw it at a constant magnitude that equals the attraction in the bulk. As a result, there indeed is a net attraction of the subsystem by the rest of the liquid (dark gray arrow in Fig. 6.7c).

\subsubsection{The liquid-solid interface}

\section{Forces near the liquid-solid interface}

We now consider the liquid-solid interface (see Fig. 6.8). Here, two effects superimpose. First, due to the presence of the solid, there is a lack of liquid in the lower half-space (hatched region in Fig. 6.8). This missing liquid induces an anisotropy of the attractive liquid-liquid force in the same way as it does in the case of the liquid-vapor interface. Therefore, as in Fig. 6.7c, the left hand side of the liquid exerts a net attractive force $\gamma_{\mathrm{LV}}$ per unit length on the right hand side subsystem. The second effect is due to the liquid-solid interaction. In the same way as with the liquid-vapor interface, we divide the liquid into two subsystems using a control surface parallel to the interface, as shown in Fig. 6.8a. The attraction by the solid (gray arrow) decreases with the distance and is perfectly balanced by a short range liquidliquid repulsive force (dashed arrows). Now, we divide the liquid into two subsystems using a control surface perpendicular to the liquid solid interface (Fig. 6.8b). Assuming once more that the liquid-liquid repulsion is isotropic, the left part of the liquid exerts a net repulsive force on the right subsystem (dotted region). This force is induced by the influence of the solid and, perhaps surprisingly, it is not equal to $\gamma_{\mathrm{SL}}$. Instead, it has been shown[11] that this force is equal to $\gamma_{\mathrm{SV}}+\gamma_{\mathrm{LV}}-\gamma_{\mathrm{SL}}$, and this will be motivated in more 

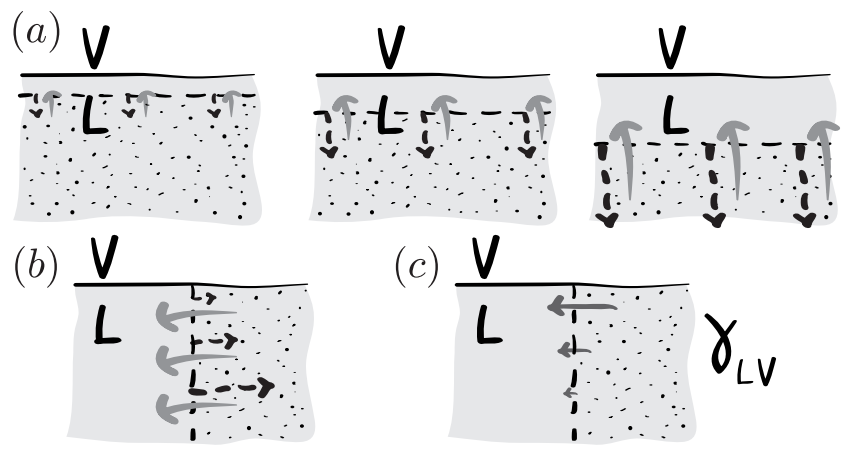

Figure 6.7: Forces exerted on a subsystem of liquid (dotted region) by the rest of the liquid (gray region without dots) (a) The subsystem considered is the lower part of the liquid and is separated from the interfacial zone by a line parallel to the liquidvapor interface. The subsystem (dotted region) is submitted to an attractive force (gray arrows) and a repulsive force (dashed black arrows) exerted by the rest of the liquid (gray region without dots). They must balance each other. (b) The liquid is now divided along a line perpendicular to the interface. The subsystem considered (dotted region on the right) is submitted to an attractive force (gray arrows) and to a repulsive force (dashed arrows) exerted by the rest of the liquid (gray region without dots).As the repulsive force is isotropic, it has the same magnitude as in (a) and therefore decays close to the surface. On the contrary, the attractive force is nearly constant and remains almost unchanged close to the surface. (c) This leads to a net attractive force from one side on the other.

detail below.

To combine these two effects, we subtract the unbalanced attractive force due to the absent liquid $\left(\gamma_{\mathrm{LV}}\right)$ from the repulsive force due to the solid $\left(\gamma_{\mathrm{SV}}+\right.$ $\left.\gamma_{\mathrm{LV}}-\gamma_{\mathrm{SL}}\right)$ to find the net repulsive force between the subsystems: $\gamma_{\mathrm{SV}}-\gamma_{\mathrm{SL}}$.

\section{Solid-liquid interaction and the surface tensions}

Let us motivate why the strength of the solid-liquid interaction does not couple directly to the solid-liquid surface tension $\gamma_{\mathrm{SL}}$, but to the combination $\gamma_{\mathrm{SV}}+\gamma_{\mathrm{LV}}-\gamma_{\mathrm{SL}}[1]$. Note that this feature will also be crucial for understanding the wetting phenomena discussed in the following section.

The solid-liquid surface tension represents the free energy needed to create a solid-liquid interface. To make such an interface, one first has to "break" a bulk solid and a bulk liquid into two separate parts, and then join these solid and liquid parts together. This breaking of liquid is de- 


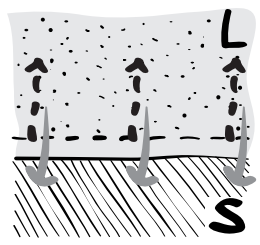

(a)

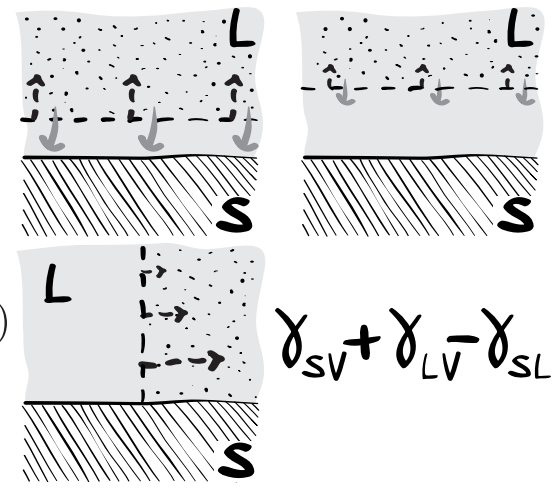

Figure 6.8: Forces exerted by the solid (dashed line) on a subsystem of liquid (dotted region). On these schematics, the attractive liquid-liquid interactions already treated in Fig. 6.7, are not considered. (a) The liquid subsystem is semi-infinite. It is delimited by a line parallel to the liquid-solid interface, at different distances above it. The subsystem (dotted region) is submitted to an attractive force (gray arrows) exerted by the solid and to a repulsive force (dashed arrows) exerted by the rest of the liquid (gray region without dots). As the subsystem is in equilibrium, they must balance each other. (b) The liquid is now divided along a line perpendicular to the interface. Only the horizontal force components are shown. The solid exerts no horizontal attraction. As the repulsive interactions are isotropic, this results into an horizontal repulsive force exerted by one side on the other.

picted schematically in Fig. 6.9a (it works similarly for the solid). The corresponding energy is the "work of adhesion" $A_{\mathrm{LL}}$ due to liquid-liquid attractions ( $A_{\mathrm{SS}}$ for the solid). This gives rise to a surface tension $2 \gamma_{\mathrm{LV}}=A_{\mathrm{LL}}$ $\left(2 \gamma_{\mathrm{SV}}=A_{\mathrm{SS}}\right)$, since the liquid (solid) is connected to a vacuum at this intermediate stage. When joining the solid-vacuum and liquid-vacuum interfaces, the attractive solid-liquid interaction will reduce the surface energy by the solid-liquid work of adhesion $A_{\mathrm{SL}}$ (Fig. 6.9b). Hence, the resulting solid-liquid surface tension becomes

$$
\gamma_{\mathrm{SL}}=\gamma_{\mathrm{SV}}+\gamma_{\mathrm{LV}}-A_{\mathrm{SL}} .
$$

From this one finds that, indeed, the strength of the solid-liquid adhesion reads

$$
A_{\mathrm{SL}}=\gamma_{\mathrm{SV}}+\gamma_{\mathrm{LV}}-\gamma_{\mathrm{SL}}=\gamma_{\mathrm{LV}}(1+\cos \theta) .
$$

In the last step we used Young's law for the equilibrium contact angle. As 


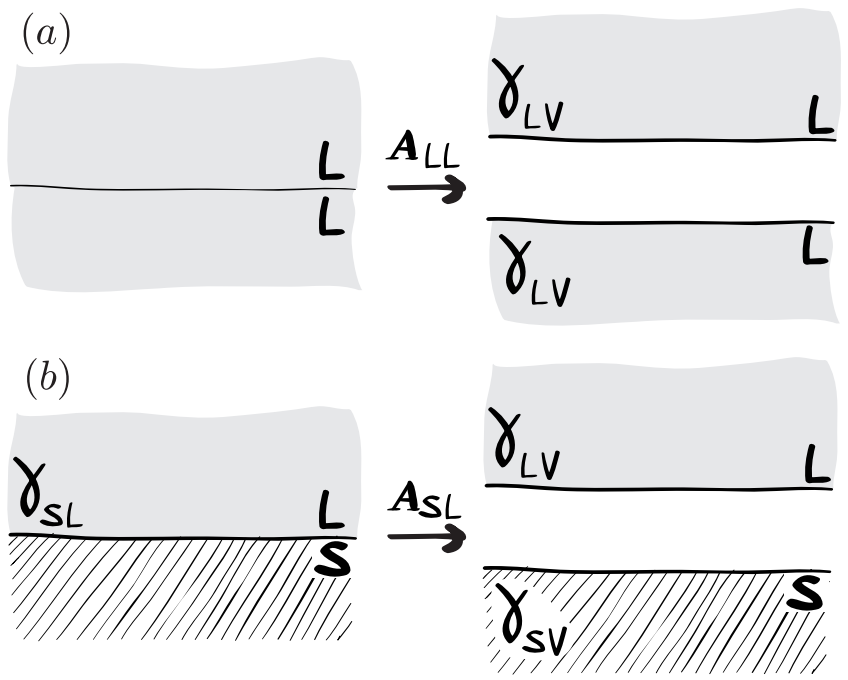

Figure 6.9: Relation between adhesion work and surface tensions. (a) To split a liquid volume into two semi-infinite volume, one has to create two liquid-vacuum interfaces. It costs an energy $A_{\mathrm{LL}}=2 \gamma_{\mathrm{LV}}$. (b) To create a solid-liquid interface one first needs to create a liquid-vacuum and solid-vacuum interface, which costs an energy $\gamma_{\mathrm{SV}}+\gamma_{\mathrm{LV}}$. Joining the liquid-vacuum and solid-vacuum interfaces yields an energy reduction $A_{\mathrm{SL}}=\gamma_{\mathrm{SV}}+\gamma_{\mathrm{LV}}-\gamma_{\mathrm{SL}}$ due to the solid-liquid attraction.

a consequence, the capillary forces induced by solid-liquid attractions will have a magnitude $A_{\mathrm{SL}}=\gamma_{\mathrm{LV}}(1+\cos \theta)$ and not $\gamma_{\mathrm{SL}}$.

\subsection{Microscopic interpretation of wetting}

The question of the force balance is even more intricate in the vicinity of the contact line, where the liquid-vapor interface meets a solid. It is crucial to note that the contact line itself does not represent any material: it is a mathematical line that marks the separation between wetted and dry parts of the solid. The question "What is the force on the contact line?" is thus ill-posed, since there are no molecules on which such a force would act. As a matter of fact, only a material system (a collection of matter) can be submitted to a force. Therefore, care should be taken to properly define the systems that play a role near the contact line: the liquid near the contact line and the solid underneath it. In the following sections we will show how a careful 


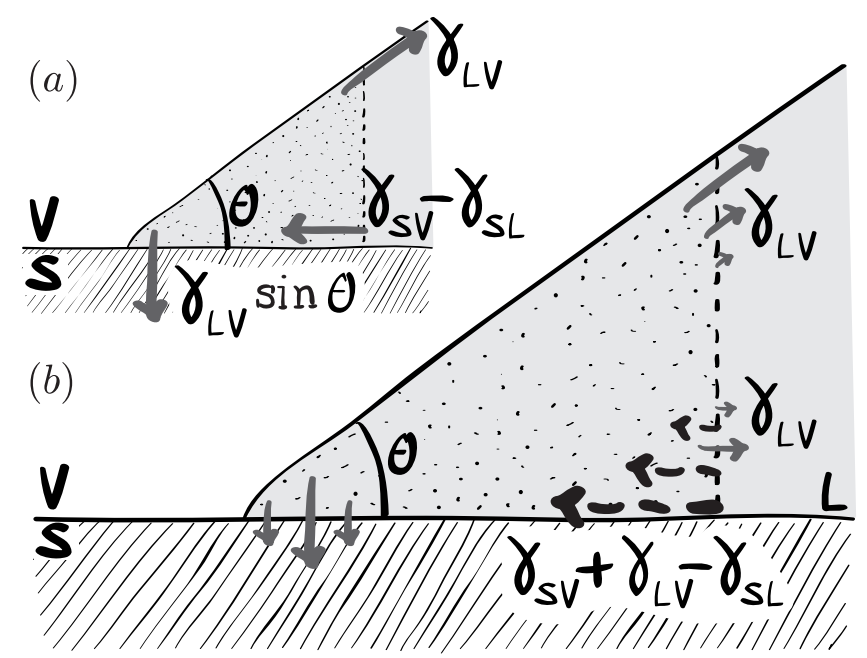

Figure 6.10: Solid and liquid forces acting on a liquid subsystem (dotted regions) near the contact line. (a) Sketch of a wedge of liquid near the contact line with the three forces exerted on the system. (b) Each of the three corners of this system must be treated differently. The upper right corner is at the liquid vapor interface. Following Fig. 6.7, the rest of the liquid exert a net attractive force parallel to the interface equal to $\gamma_{\mathrm{LV}}$ per unit length. The lower right corner is at the liquid solid interface. Following Figs. 6.7 and 6.8, the rest of the liquid exerts a repulsive force $\gamma_{\mathrm{SV}}-\gamma_{\mathrm{SL}}$. The liquid near the solid-liquid interface is attracted by the solid, this force is balanced everywhere by repulsion at the solid-liquid interface, except in the vicinity of the contact line.

consideration of all the forces on the appropriate material systems will lead to proper force balances, consistent with the thermodynamic predictions.

All results and sketches provided in this section, some of which may appear counterintuitive, are backed up by a Density Functional Theory model for microscopic interactions $[12,13]$ and molecular dynamics simulations, see e.g. chapter 7 .

\subsubsection{Force on a liquid corner: Question 2}

Let us consider the forces on the wedge-shaped liquid corner in the vicinity of the contact line, as shown in Fig. 6.10. Choosing this subsystem, we will now explain Young's force construction from Fig. 6.3 b and answer Question 2: What happens to the force balance normal to the solid-liquid interface? 
There are two types of forces acting on the liquid molecules inside the subsystem: interactions with the solid and interactions with other liquid molecules outside the subsystem. We first consider the solid-on-liquid forces. One can immediately see that, since the solid spans an infinite half space, every liquid molecule experiences a resultant force that is oriented purely normal to the solid-liquid interface: the left-right symmetry of the solid ensures that there is no force component parallel to the interface. Far from the contact line at the solid-liquid interface, this attractive force is balanced by a repulsive force, as discussed in Fig. 6.8. However, since the repulsive force is continuous and zero outside the droplet, the repulsive force must decay close to the contact line. This means that there is an unbalanced attractive force that is strongly localized in the vicinity of the contact line. It has been shown[14] that this force per unit length is equal to $\gamma_{\mathrm{LV}} \sin \theta$. The existence of this force has recently been challenged $[15,16]$. To show that this force indeed must exist to achieve equilibrium, we consider the droplet shown in Fig. 6.11. Choosing the drop as the system, and recognizing that the force in the interior of the droplet at the liquid-solid interface (small arrows) is due to the Laplace pressure $2 \gamma_{\mathrm{LV}} \mathcal{K}$ (with $\kappa$ the curvature $1 / R$ ), the attractive force at the contact line must be exactly $\gamma_{\mathrm{LV}} \sin \theta$ to achieve a force balance[17-21]. This provides the answer to Question 2: the downward solid-on-liquid force is not drawn in Fig. 6.3b. This missing force has often been interpreted as a reaction from the solid [2], whose existence is demonstrated experimentally by the elastic deformation of soft solids below the contact line[20-23]. Here, we clarify the molecular origin of this normal force [14].

To finalize the force construction near the contact line we return to the wedge shown in Fig. 6.1ob. Since the solid can only exert a normal force on the liquid, all parallel force components drawn in Young's construction are purely due to the liquid molecules outside the corner. The force drawn along the liquid-vapor interface can be understood directly from the tension $\gamma_{\mathrm{LV}}$ inside the liquid-vapor interface (cf. the discussion of Fig. 6.7). A similar force arises at the solid-liquid interface (Fig. 6.8), which, however, is repulsive and has a magnitude $\gamma_{\mathrm{SV}}-\gamma_{\mathrm{SL}}$, as explained in the previous section. Including these forces gives a perfect force balance on the liquid corner, as seen in Fig. 6.10a. In fact, one can easily verify that even the resultant torque (or force moment) is zero for this force construction. As such, it provides a more physical alternative to the classical picture of Young's law. 


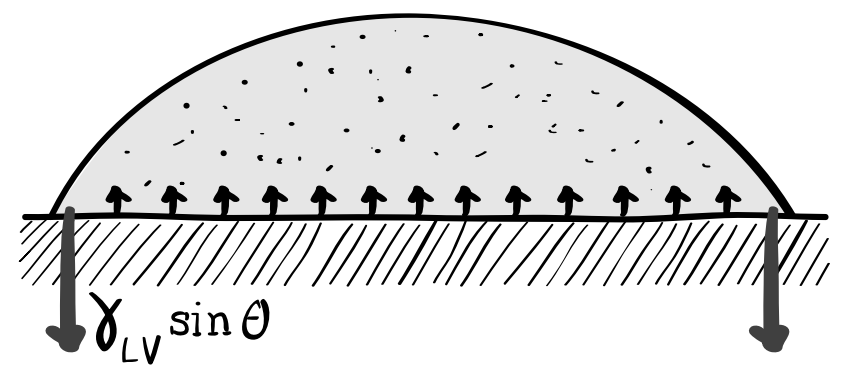

Figure 6.11: Forces acting on a system, in this case a liquid drop (dotted area). The system is in equilibrium so the sum of all (external) forces must be zero. Due to Laplace pressure, there is a repulsive force exerted by the solid on the liquid across the liquid-solid interface (upward black arrows). In the vicinity of the contact line, repulsion and attraction of the liquid by the solid do not balance each other. Therefore, the solid attracts the liquid with a vertical force equal to $\gamma_{\mathrm{LV}} \sin \theta$ per unit length (downward dark gray arrows).

\subsubsection{Liquid-on-Solid force: Question 3}

The measurement of surface tension in Fig. 6.3a directly relies on the force exerted by the liquid on the solid plate. Again, we emphasize the importance of a proper definition of "the system" on which the forces act, and in this case this is the solid on which the liquid rests. The situation is thus very different from the forces on the liquid corner, which are in equilibrium and experiences a zero resultant force. This difference between systems provides the key to Question 3. In Fig. 6.3a, the total force exerted by the liquid on the solid is represented by the resultant $\vec{\gamma}_{\mathrm{LV}}$, while Fig. $6.3 \mathrm{~b}$ represents the balance of the forces acting on the liquid wedge.

\section{Forces near the contact line}

Once again, we turn to the microscopic description of the forces in the vicinity of the contact line. It turns out that the normal component of the force exerted on the solid is equal to $\gamma_{\mathrm{LV}} \sin \theta$, consistent with the macroscopic picture of a tension along the liquid-vapor interface. However, the parallel component of the liquid-on-solid force does not have the expected magnitude $\gamma_{\mathrm{LV}} \cos \theta$, but $\gamma_{\mathrm{LV}}+\gamma_{\mathrm{SV}}-\gamma_{\mathrm{SL}}=\gamma_{\mathrm{LV}}(1+\cos \theta)$. This can be understood as follows. Fig. 6.12a illustrates that the tangential force component originates from the long-range attraction between solid and liquid molecules. In the 


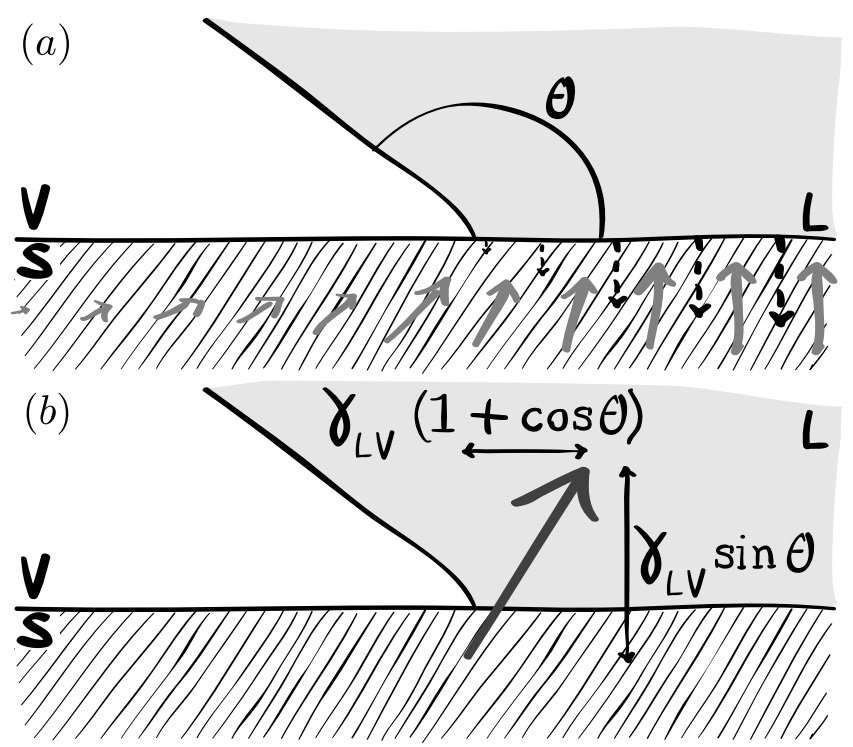

Figure 6.12: Forces acting on the solid subsystem (hatched areas) by the liquid (gray areas) near the contact line. (a) Distribution of forces acting on the solid near the contact line. Due to the attraction of the liquid, the solid is attracted towards the liquid (solid gray arrows). The absence of liquid on the left part of the contact line ensures the tangential force is always towards the liquid, even for $\theta>90^{\circ}$. The repulsion (dotted arrows) arises from the contact force at the solid-liquid interface. Far from the contact line, repulsion and attraction balance each other. (b) Resultant force acting on the solid near the contact line. The net normal force is $\gamma_{\mathrm{LV}} \sin \theta$, and the parallel force $\gamma_{\mathrm{LV}}+\gamma_{\mathrm{SV}}-\gamma_{\mathrm{SL}}=\gamma_{\mathrm{LV}}(1+\cos \theta)$.

previous section we already demonstrated the strength of this solid-liquid adhesion to be $A_{\mathrm{SL}}=\gamma_{\mathrm{LV}}(1+\cos \theta)$. Hence, there is no reason why the total force on the solid should be $\gamma_{\mathrm{LV}} \cos \theta$. Indeed, the DFT calculation confirms a tangential liquid-on-solid force of magnitude $A_{\mathrm{SL}}=\gamma_{\mathrm{LV}}(1+\cos \theta)$ [14].

The physics of this surprising result is nicely illustrated by Fig. 6.12. The macroscopic intuition that the resultant surface tension force is pulling along the liquid-vapor interface would predict a force to the left whenever the contact angle $\theta>90^{\circ}$. However, it is clear from the sketch of the attractive forces that the sum of all parallel components must be oriented towards the liquid (right side in the figure). This stems from the asymmetry between the amount of liquid attracting the solid molecules on both sides of the contact line: there are always more liquid molecules on the right side of the contact 
(a)

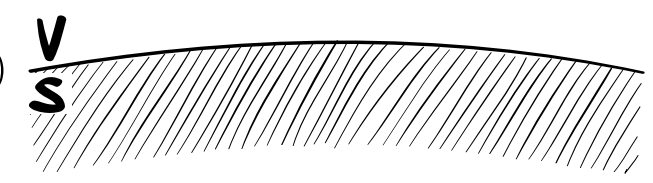

(b)
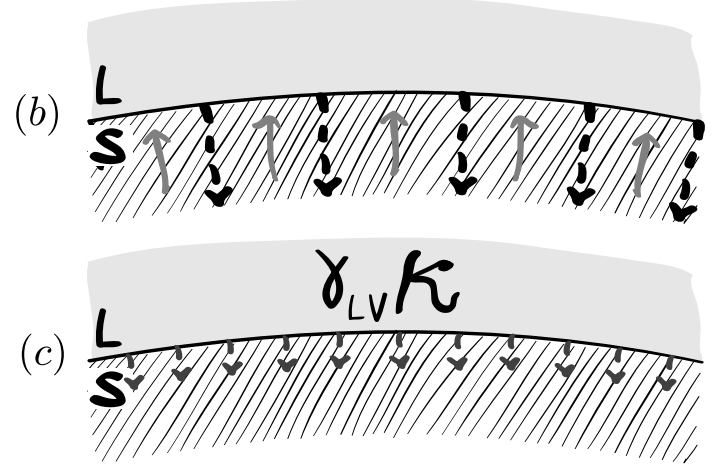

Figure 6.13: Forces acting on a solid at the solid-liquid interface. (a) Without liquid, there is neither repulsion nor attraction. (b) When liquid is present there is repulsion and attraction. However, the repulsion is not completely balanced in this curved interface, as there is more liquid than in a plane geometry. (c) The resulting force per unit surface is $\gamma_{\mathrm{LV}} \kappa$ where $\kappa$ is the curvature of the liquid (positive in this case), equivalently to the Laplace pressure. It only shows a dependency on the liquid-liquid interactions as it is only the absence of liquid that is relevant to the curvature.

line in this figure. This is consistent with a parallel force $\gamma_{\mathrm{LV}}(1+\cos \theta)$, but not with a force $\gamma_{\mathrm{LV}} \cos \theta$ (which changes sign at $90^{\circ}$ ). Note that when considering the force exerted by the solid on the liquid, this asymmetry does not occur since the solid is left-right symmetric, therefore there is no tangential component. This once more illustrates that a detailed force interpretation crucially relies on the definition of the system.

\section{Global force balance: curvature of solid-liquid interface}

To solve this apparent discrepancy with the thermodynamic result one has to consider all the forces exerted by the liquid on the solid, i.e. not just the forces near the contact line. The key point is that the submerged solid bodies cannot be flat everywhere and must have curved pieces of liquid-solid interface. If the interface separating the solid from the liquid is flat, the net normal force is locally zero as repulsion balances attraction (far away 
from the contact line). However, when this interface is curved, a repulsive force inside the liquid is enhanced due to the curvature of the solid-liquid interface, in a way similar to the Laplace pressure. As shown in Fig. 6.13, the presence of a curved half-space of liquid acts on the solid, and creates an unbalanced liquid-on-solid force $\gamma_{\mathrm{LV}} \mathcal{K}$ per unit area. Here $\kappa$ is the local curvature (inverse radius of curvature) of the solid. Interestingly, DFT calculations [14] show that the resultant pressure couples only to $\gamma_{\mathrm{LV}}$ and not to $\gamma_{S L}$. As we show below, this is exactly what is needed to restore consistency between microscopic and thermodynamic forces.

An excellent demonstration of this effect is the long debated case of a 'floating-pin' under zero-gravity, as shown in Fig 6.14. Whereas a floating pin in a system with gravity leaves a visible depression in the liquid-vapor interface near the contact line (cf. Fig. 6.14e), the zero-gravity condition ensures that the interface has a constant curvature, i.e. it is straight everywhere. This means that the vertical position depends on the equilibrium contact-angle alone, and not on the density ratio of the involved materials. As discussed in Fig. 6.12, the liquid-on-solid force near the contact line is not oriented along the liquid-vapor interface, but points towards the interior of the liquid. This creates a resultant downward force that has to be compensated to restore equilibrium. Additionally, the curvature of the solid-liquid interface creates a normal force distributed over the whole immersed surface of the solid of magnitude $\gamma_{\mathrm{LV}} \kappa$ per unit surface (cf. Fig. 6.14a). Integrating over the curvature of the submerged surface from one contact line to the other then gives the resultant of the Laplace pressure:

$$
\gamma_{\mathrm{LV}} \int_{1}^{2} \kappa \vec{n} d S=\gamma_{\mathrm{LV}}\left(\vec{t}_{2}+\vec{t}_{1}\right)
$$

where $\vec{t}_{1}$ and $\vec{t}_{2}$ are the unit vectors tangential to the pin, pointing upwards. Therefore, the resultant is orientated upwards and is equal to $2 \gamma_{\mathrm{LV}} \sin \theta$ per unit depth (see Fig. 6.14b). It balances exactly the downward forces induced close to the contact lines (Fig. 6.12b), hence the pin is in equilibrium. Importantly, this result is independent of the shape of the body (see Fig. 6.14d).

The same principle applies to the partially wetted plate of Fig. 6.3a: the force exerted by the fluid on the plate results from two contributions, as shown schematically in Fig. 6.15c. First, there is the vertical force component (per unit length) due to the vicinity of the contact line: $\gamma(1+\cos \theta)$ (cf. Fig. 6.12). Second, there are submerged surfaces of the plate where a localized curvature exists at the corners. This curvature induces a Laplace force 


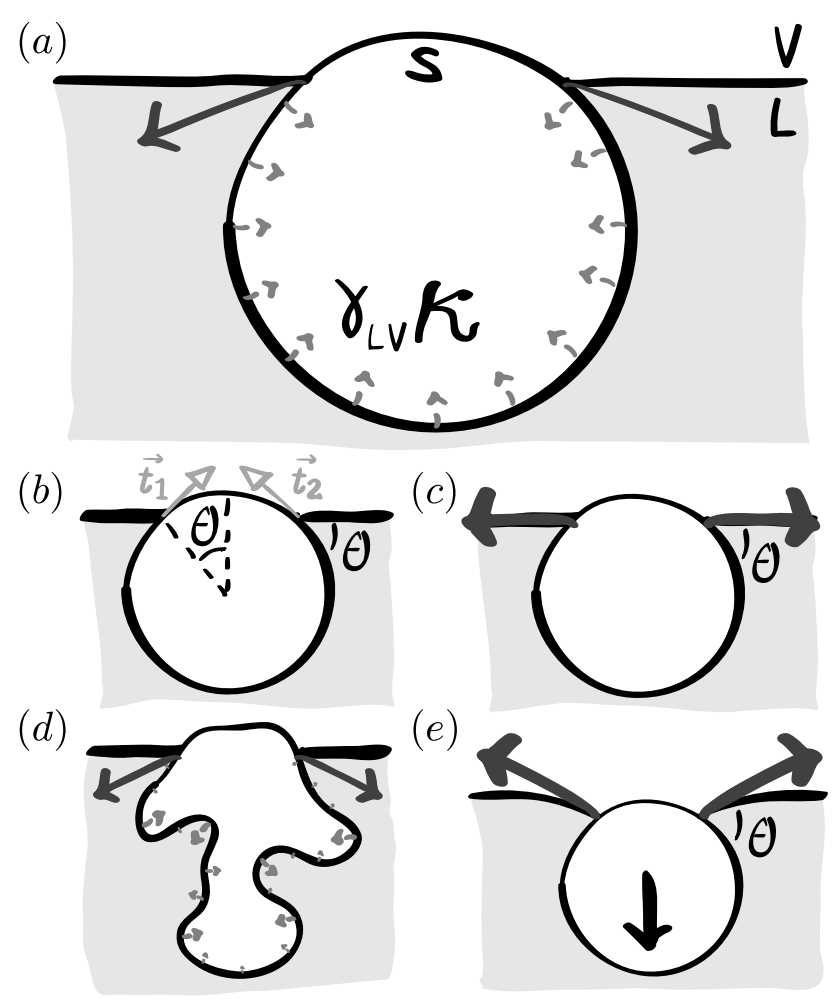

Figure 6.14: Capillary forces on immersed objects. (a) Schematic of a pin floating at the surface of a liquid under partial wetting conditions and in zero-gravity. The downward thin gray arrows are the forces exerted by the liquid on the pin located in the vicinity of the contact line. The small light gray arrows show the Laplace pressure $\gamma_{\mathrm{LV}} \kappa$ acting on the solid, due to the curvature $\kappa$ of the solid-liquid interface. (b) $\theta$ denotes the contact angle and $\vec{t}_{1}$ and $\vec{t}_{2}$ are two unit vectors tangential to the pin, pointing upwards, at the two contact lines. (c) The thick gray arrows show the resultants of the capillary forces detailed in (a), which apply on each half of the pin. They reduce to forces tangential to the liquid-vapor interface at the contact lines. Note that this schematic does not show the distribution of capillary forces. (d) Distribution of the capillary forces in the case of an irregular shape. Since the integral over the curvature is equal to the sum of the two tangential vectors at the contact lines, the resultant is independent of the shape of the body. It is thus the same as in (c). (e) Pin floating at the surface of a liquid under gravity. The upward thick gray arrows are the resultants of capillary forces. They balance the effect of gravity (corrected by the Archimedes force), shown as the downward black arrow. 
on the pin (see Fig. 6.14d) which results into a net upward force $\gamma_{\mathrm{LV}}$ per unit length of contact line which means the total force (per unit length of contact line) on the plate is $\gamma_{\mathrm{LV}} \cos \theta$, in agreement with the thermodynamic result.

\subsubsection{Complete wetting: Question 4}

In the case of complete wetting, Young's law for the contact angle is no longer applicable. Instead, the apparent contact angle $\theta$ vanishes because the three surface tensions do not balance each other:

$$
\gamma_{\mathrm{SV}}-\gamma_{\mathrm{SL}}>\gamma_{\mathrm{LV}}
$$

Physically, there is no real contact line in this configuration (Fig. 6.15b), but there is a meniscus where the liquid-vapor interface approaches the solid. Beyond the meniscus, there exists a mesoscopic liquid film called a prewetting film, which covers the solid completely (Fig. 6.15d). The existence of an apparent contact line is only due to the effect of gravity: on a flat surface, the liquid would simply spread. The interface between the liquid and vapor phases consequently presents two regions. In the lower region, the meniscus can be described by the balance between the Laplace pressure and the gravity potential

$$
\gamma_{\mathrm{LV}} \kappa=\rho g z,
$$

where $z$ is the height above the bath (thus, no additional constant needed) and $\kappa$ the curvature of the interface. Introducing the capillary length $\ell_{\gamma}=$ $\sqrt{\gamma_{\mathrm{LV}} / \rho g}$ this equation can be written as: $\ell_{\gamma}^{2} \kappa=z$. In the upper region, there is the pre-wetting film whose thickness $h(z)$ is determined by the balance between the gravity potential and the disjoining pressure $\Pi(h)$. The disjoining pressure is by definition the potential energy per unit volume at the surface of a liquid layer of thickness $h$, therefore the balance reads[1]:

$$
\Pi(h)=\rho g z .
$$

As the pre-wetting film is very flat, the contribution of Laplace pressure can be neglected $(\kappa=0)$ in this regime. The disjoining pressure scales as:

$$
\Pi(h) \simeq \frac{\left(\gamma_{\mathrm{SV}}-\gamma_{\mathrm{SL}}-\gamma_{\mathrm{LV}}\right) \sigma^{2}}{h^{3}},
$$

for films where $h \gg \sigma$, where $\sigma$ is a length on the order of the molecular size. The disjoining pressure vanishes in the limit case $\gamma_{\mathrm{SV}}=\gamma_{\mathrm{SL}}+\gamma_{\mathrm{LV}}$, which can 

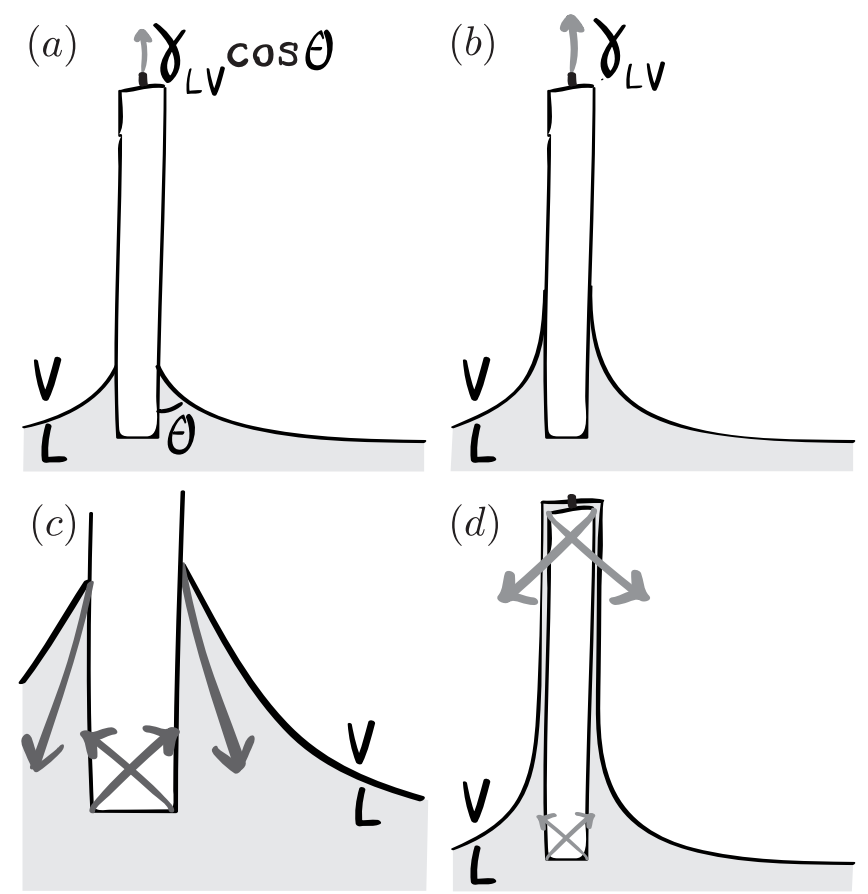

Figure 6.15: $(a-b)$ Force per unit length of the contact line needed to keep a plate in equilibrium in a bath in partial (a) and complete wetting (b). (c) Partial wetting. The vertical force at the contact line, equal to $\gamma_{\mathrm{LV}}(1+\cos \theta)$, is balanced by the Laplace pressure induced by the curvature of the plate. Note that any plate shape would lead to the same resultant force as the integral of the curvature over the surface reduces to the local tangents at the contact line (see Fig. 6.14d). (d) Complete wetting case. Due to the mesoscopic pre-wetting film, of which the thickness is exaggerated in the picture, there is no contact line, thus there is no force located near the apparent macroscopic contact line. The forces are related only to the Laplace pressure: the curvature of the solid gives a zero resultant force, as it is completely immersed in the liquid, and the curvature of the liquid is acting on the solid only in the pre-wetting zone - as it is compensated by gravity in the meniscus. The resultant force per unit length is then equal to $\gamma_{\mathrm{LV}}$. 
be interpreted as the situation for which the interaction is the same with the liquid and with the solid. Then, one indeed does not expect any influence of the thickness $h$ on the energy.

Equating gravity and disjoining pressure (eq. 6.9), we obtain the thickness profile in the pre-wetting region:

$$
h(z) \simeq\left[\frac{\left(\gamma_{\mathrm{SV}}-\gamma_{\mathrm{SL}}-\gamma_{\mathrm{LV}}\right) \sigma^{2}}{\rho g z}\right]^{1 / 3}
$$

In the vicinity of the apparent contact line, where the two zones must match, the thickness is thus of order $l_{\gamma}^{1 / 3} \sigma^{2 / 3}$. As $l_{\gamma}$ is millimetric and $\sigma$ nanometric, $h$ is mesoscopic ( $h \simeq 100 \mathrm{~nm}$ ). From the microscopic point of view, the solid is then completely surrounded by a semi-infinite layer of liquid $(h \gg \sigma)$. Therefore, the only forces acting on a solid in complete wetting are normal contact forces, such as Laplace pressures. There are no contact line forces such as those described in Fig. 6.12b.

The forces exerted by the liquid on the solid are then related, as previously, to the curvature of the liquid-solid interface but also, inside the pre-wetting film, to the curvature of the liquid-vapor interface (Fig 6.15d). Integrated over the whole submerged solid, the curvature of the solid gives a zero resultant force, whereas the curvature of the liquid is only integrated where the pre-wetting film exists. As a result, the resultant force is vertical and has an amplitude $\gamma_{\mathrm{LV}}$ per unit length of the apparent contact line.

This result is indeed consistent with the thermodynamic perspective. Since the solid is covered by a liquid layer much thicker than the molecular size the surface tension above the apparent contact line is not $\gamma_{S V}$ but $\gamma_{\mathrm{SL}}+\gamma_{\mathrm{LV}}$ : the plate is always completely submerged. In essence, this means that the plate never leaves the liquid bath when the plate is pulled upwards. When moving, there is no change of the solid-vapor interfacial area (it remains zero) or of the solid-liquid interfacial area (which is simply the total area of the plate). The only change occurs at the liquid-vapor interfacial area, which is increased, and the required pulling strength is thus $\gamma_{\mathrm{LV}}$ per unit length of the apparent contact line. 


\subsection{Conclusion}

\subsubsection{Summary}

In this article we have raised simple questions about capillarity that many students face. By studying the interfaces from a microscopic perspective, we were able to provide answers to these questions, and also reconcile thermodynamics and statistical physics.

We have provided a mechanical perspective about why there exists an attractive force parallel to interfaces: surface tension. The absence of liquid creates an attractive anisotropic force within a few molecular lengths from the interface whereas the repulsion remains isotropic and scales with the local density of the fluid. The attractive anisotropy leads to a strong localized stress parallel to the interface called surface tension. This occurs at liquidsolid interfaces as well, where there is also a half-space of liquid missing.

The problems when constructing force pictures in capillarity often arise from the improper definition of a system on which the forces act. Considering a corner of liquid near the contact line as a system, we have proposed here an alternative to Young's construction (Fig. 6.3b). The analysis allows to locate and understand the different forces, in particular the attractive force exerted by the solid. This new force constructions leads to a perfect mechanical equilibrium: vectorial force balance and torque balance.

When looking at the force that is exerted by the liquid on the solid near the contact line we find that, surprisingly, this force is not $\gamma_{\mathrm{LV}} \cos \theta$, but $\gamma_{\mathrm{LV}}(1+\cos \theta)$. Moreover, a normal stress is exerted in all the regions of any curved solid-liquid interface: the liquid pulls the solid when the latter is convex. This force is equivalent to the usual Laplace pressure. One has to take both these forces into account, to obtain the net force from the thermodynamic result. The advantage of this microscopic force description is that it provides a simple answer to an academic problem which has become controversial in the last decade: the floating pin paradox $[15,16,24]$.

The drawings and several relations presented in this chapter are based on results obtained using the Density Functional Theory in the sharp-kink approximation[14], which allows to make quantitative predictions of the force distributions in the liquid and in the solid. . 


\subsubsection{Teaching perspective}

We realize that the detailed picture of microscopic forces is not necessarily the most accessible for teaching purposes. In particular when introducing the basic concepts of capillarity, it is much simpler to work from the thermodynamic perspective: energy minimization naturally yields the equilibrium conditions, while the resultant forces can be computed from the virtual work principle. Nevertheless, our analysis provides a number of insights that are useful when teaching capillarity:

- To determine "capillary forces" it is crucial to explicitly specify the system (= collection of matter) to which the forces are applied.

- The surface tensions $\gamma_{\mathrm{SL}}$ and $\gamma_{\mathrm{SV}}$ do not pull on the solid.

- The global force exerted on the solid by the liquid can be computed by adding the contributions of (Laplace) pressure inside the liquid and a localized tension force $\gamma_{\mathrm{LV}}$ parallel to the liquid-vapor interface. While this gives the correct answer, it does not reflect the true microscopic distribution of liquid-on-solid forces.

- By contrast, the resultant force on the liquid near the contact line does involve the three surface tensions $\gamma_{\mathrm{LV}}, \gamma_{\mathrm{SL}}$ and $\gamma_{\mathrm{SV}}$.

- The classical construction of Fig. 6.3b to explain Young's law does not accurately represent the force balance. A complete picture is provided in Fig. 6.10a.

We hope in particular that the force construction of Fig. 6.10a will find its way to the classroom to explain Young's law (6.2). It is conceptually simple, it clarifies the "system" to which forces are applied, and represents a perfect mechanical equilibrium. Namely, besides a balance of normal and tangential components, the forces also exert a zero torque.

Finally, we note that the virtual work principle yields the correct resultant force on a solid, but that it cannot recover the true microscopic force distribution. The knowledge of such a force distribution is crucial when one wants to take into account how a solid is elastically deformed by the contact line [17-19]. Even though these deformations can be as small as a few nanometers, these can be measured due to the advent of modern experimental techniques [20-23]. This experimental access renews the fundamental interest in the microscopic details of capillarity [14]. 


\section{References}

[1] P.-G. de Gennes, Wetting - Statics and Dynamics, Rev. Mod. Phys. 57, 827 (1985).

[2] P.-G. de Gennes, F. Brochard-Wyart, and D. Quéré, Capillarity and Wetting Phenomena (Springer, New York, 2004).

[3] D. Bonn, J. Eggers, J. Indekeu, J. Meunier, and E. Rolley, Wetting and spreading, Rev. Mod. Phys. 81, 739 (2009).

[4] J. Kirkwood and F. Buff, The Statistical Mechanical Theory of Surface Tension, J. Chem. Phys. 17, 338 (1949).

[5] J. S. Rowlinson and B. Widom, Molecular Theory of Capillarity (Dover publications, Mineola, NY, 1982).

[6] M. Berry, The Molecular mechanism of surface tension, Physics Educations 6, 79 (1971).

[7] J. W. Gibbs, The Collected Works of J. Willard Gibbs (Yale University Press, London, 1957), p. 288.

[8] T. Young, An Essay on the Cohesion of Fluids, Philos. Trans. 95, 65 (1805).

[9] J.-P. Hansen and I. R. McDonald, Theory of Simple Liquids (Academic Press, Waltham, MA, 2006).

[10] J. Indekeu, Line Tension near the Wetting Transition - Results from an Interface Displacement Model, Physica A 183, 439 (1992).

[11] M. Nijmeijer, C. Bruin, A. Bakker, and J. Van Leeuwen, Wetting and drying of an inert wall by a fluid in a molecular-dynamics simulation, Phys. Rev. A 42, 6052 (1990).

[12] C. Bauer and S. Dietrich, Quantitative study of laterally inhomogeneous wetting films, Eur. Phys. J. B 10, 767 (1999).

[13] T. Getta and S. Dietrich, Line tension between fluid phases and a substrate, Phys. Rev. E 57, 655 (1998).

[14] S. Das, A. Marchand, B. Andreotti, and J. H. Snoeijer, Elastic deformation due to tangential capillary forces, Phys. Fluids 23, 072006 (2011). 
[15] R. Finn, The contact angle in capillarity, Phys. Fluids 18, 047102 (2006).

[16] R. Finn, Comments related to my paper "The contact angle in capillarity", Phys. Fluids 20, 107104 (2008).

[17] A. Rusanov, Theory of wetting of elastically deformed bodies. 1. Deformation with a finite contact angle, Coll J. USSR 37, 614 (1975).

[18] A. Carre, J. Gastel, and M. Shanahan, Viscoelastic effects in the spreading of liquids, Nature 379, 432 (1996).

[19] L. R. White, The contact angle on an elastic substrate. 1 . The role of disjoining pressure in the surface mechanics, Journal of Colloid and Interface Science 258, 82 (2003).

[20] R. Pericet-Camara, G. K. Auernhammer, K. Koynov, S. Lorenzoni, R. Raiteri, and E. Bonaccurso, Solid-supported thin elastomer films deformed by microdrops, Soft Matter 5, 3611 (2009).

[21] R. Pericet-Cámara, A. Best, H.-J. Butt, and E. Bonaccurso, Effect of Capillary Pressure and Surface Tension on the Deformation of Elastic Surfaces by Sessile Liquid Microdrops: An Experimental Investigation, Langmuir 24, 10565 (2008).

[22] Y. Wang, B. Bhushan, and X. Zhao, Nanoindents produced by nanobubbles on ultrathin polystyrene films in water, Nanotechnology 20, 045301 (2009).

[23] Y.-S. Yu and Y.-P. Zhao, Elastic deformation of soft membrane with finite thickness induced by a sessile liquid droplet, Journal of Colloid and Interface Science 339, 489 (2009).

[24] I. Lunati, Young's law and the effects of interfacial energy on the pressure at the solid-fluid interface, Phys. Fluids 19, 118105 (2007). 


\section{7 \\ Origin of line tension for a Lennard-Jones nanodroplet $*+$}

The existence and origin of line tension has remained controversial in literature. To address this issue we compute the shape of Lennard-Jones nanodrops using molecular dynamics and compare them to density functional theory in the approximation of the sharp kink interface. We show that the deviation from Young's law is very small and would correspond to a typical line tension length scale (defined as line tension divided by surface tension) similar to the molecular size and decreasing with Young's angle. We propose an alternative interpretation based on the geometry of the interface at the molecular scale.

\subsection{Introduction}

The development of microfluidics in the last decade has renewed the interest for a thermodynamical concept introduced by Gibbs in his pioneering article: line tension [1]. By analogy with surface tension, which is by defi-

${ }^{*}$ Published as: J.H. Weijs, A. Marchand, B. Andreotti, D. Lohse, and J.H. Snoeijer, “Origin of line tension for a Lennard-Jones nanodroplet.", Phys. Fluids 23, 022001 (2011).

${ }^{+}$The MD simulations in this chapter are part of the present thesis. The DFT work was done by Antonin Marchand. 
nition the excess free energy per unit surface of an interface separating two phases, line tension is the excess free energy per unit length of a contact line where three distinct phases coexist. The variation of a system free energy $F$ therefore presents three contributions, a bulk contribution when the volume $V$ is varied, a surface contribution when any interface area $S_{i}$ is varied and a line contribution when the contact line length $L$ is varied:

$$
d F=P d V+\sum_{i} \gamma_{i} d S_{i}+\tau d L .
$$

Here, we use a summation to indicate that one has to take all interfaces into account (liquid-solid, liquid-vapour, and solid-vapour). The stability of deformable surfaces, such as a liquid-vapor or liquid-liquid interface, necessarily requires a positive surface tension. Although the shape of the contact line is deformable as well, the line tension cannot be inferred from a stability argument [2]. In addition, there are conceptual problems defining line tension properly $[3,4]$.

The simplest system in which a line tension effect may be observed is a liquid drop on a solid substrate, in partial wetting conditions. Consider a drop whose shape is a spherical cap characterised by its contact line radius $R$ - seen from the top - and its contact angle $\theta$. The drop volume is $V=$ $\frac{1}{3} \pi \tilde{R}^{3}\left(2-3 \cos \theta+\cos ^{3} \theta\right)$, the liquid-vapour area $S_{L V}=2 \pi \tilde{R}^{2}(1-\cos \theta)$, the solid-liquid area $S_{S L}=\pi R^{2}$ and the contact line length $L=2 \pi R$. Here we defined $\tilde{R}$ as the radius of curvature of the spherical cap: $\tilde{R}=R / \sin \theta$, see also Fig. 7.1. When minimizing the free energy with respect to $\theta$ at constant volume $(P d V=0)$, one gets [5]:

$$
\cos \theta=\frac{\gamma_{S V}-\gamma_{S L}}{\gamma}-\frac{\tau / \gamma}{R}=\cos \theta_{Y}-\frac{\tau / \gamma}{R},
$$

where $\theta_{Y}$ is the Young contact angle and $\gamma_{S L}, \gamma_{S V}, \gamma$ the solid-liquid, solidvapour, and liquid-vapour surface tension, respectively. Note that (7.2) only holds for spherical cap-shaped droplets - the contact line of a cylindershaped drop has zero curvature, which means that the contact angle $\theta$ is unaffected by line tension and independent of drop size. In this derivation, we did not take any interface curvature effects into account (such as Tolmancorrections on $\gamma$ ), if these become comparable in magnitude to line tension the measured $\tau$ from eq. 7.2 cannot be considered 'pure' line tension, but rather an apparent line tension [3,4]. From (7.2) one can see that when $\tau$ is positive, drops will present a larger contact angle than Young's angle. 


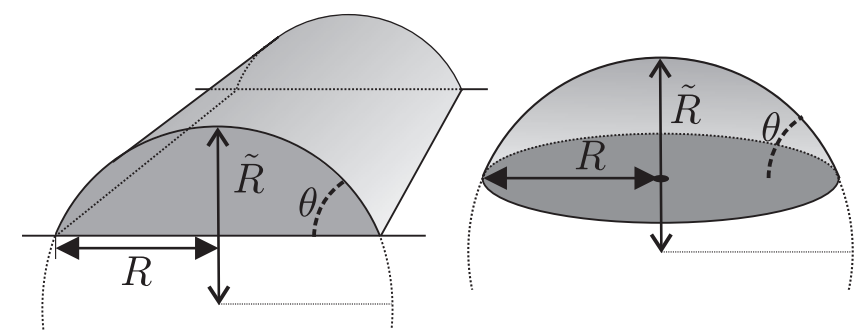

Figure 7.1: Schematic of two drops of same size, one cylindrical cap shaped (left) and the other spherical cap shaped (right). For small volumes, the contact angle $\theta$ for the spherical cap is affected by line tension, while $\theta$ is constant for the cylindrical cap.

Theoretical predictions on the strength of line tension are based on calculating the free energy (per unit length) associated with the contact line, using statistical mechanics (e.g. using density functional theory [6, 7]) or a model based on interface displacement $[8,9]$. These analyses predict the value of line tension to be in the range $10^{-12}$ to $10^{-10} \mathrm{~J} / \mathrm{m}$. Of particular interest is the behaviour near the wetting transition $(\theta \rightarrow 0)$, for which $\tau$ can vanish or diverge depending on the details of the interaction [10-13].

A large amount of experimental work has been done to determine the magnitude of line tension. The most direct way is to measure the contact angle as a function of contact line curvature, and thus droplet size [14-18]. Using the modified Young-equation from (7.2) $\tau$ can then be calculated. Due to the small length scales involved for the measurement of $\tau$, the observed values for $\tau$ vary greatly in magnitude: both negative and positive values as low as $10^{-11} \mathrm{~J} / \mathrm{m}$ and as high as $10^{-5} \mathrm{~J} / \mathrm{m}$ have been reported. The reason for the huge variation is that determining the contact angle is notoriously difficult due to contact angle hysteresis caused by surface inhomogeneities [19]. The slightest amount of surface inhomogeneities can cause a severe overestimation of $\tau$. Indeed, contaminated surfaces can even lead to an apparent change of sign of $\tau[20,21]$. Historically, droplets were used to measure line tension. Recent developments on surface nanobubbles allowed to detect a similar size dependence of the contact-angle of the nanobubbles [22-25]. Because of the difficulty of exact contact angle measurements at the required scale (1-100 $\mathrm{nm}$ ), alternative methods have been developed, for example by calculating the effective potential near the contact line by measuring the deviation of the liquid surface from a wedge shape [26, 27]. For reviews on 
experimental methods and results see refs. [11, 16].

In this chapter we adopt the usual experimental method to determine the line tension, by measuring $\theta$ against $1 / R$, in a theoretical setting. We will perform this measurement for different equilibrium contact angles $\theta_{Y}$, to study the dependence of the line tension length $(\ell)$ on $\theta_{Y}$. The tension length is defined as:

$$
\ell \equiv-\frac{\tau}{\gamma} .
$$

Since we always find negative values of $\tau$ for the Lennard-Jones drops studied in this chapter, the minus sign is added to ensure that the tension length is always positive.

We perform these measurements for both ${ }_{3} \mathrm{D}$ (spherical cap-shaped) and 2D (cylindrical-shaped) droplets, to compare similar sized droplets with and without contact line curvature. In the first part of this chapter, we investigate line tension by means of molecular dynamics simulations of a LennardJones droplet, which has the added advantage that no assumptions have to be made as is required for most analytical approaches. In this sense, these simulations are like an experiment, but with unprecedented accuracy and without surface inhomogeneity. Since the expected tension length is of the order of the molecular size, the problem also has the right scale for molecular dynamics. Line tension has been observed in molecular dynamics studies before $[28,29]$, but a systematic study has to our knowledge not been carried out. In the second part of the chapter, we analyse the existence and origin of line tension using the density functional theory (DFT) in the approximation of the sharp kink interface. Finally, we will calculate the line tension using a geometric interpretation based on missing bonds in a wedge-shaped interface. We show that the deviation from Young's law is very small and would correspond to a line tension of a fraction of the molecular size.

\subsection{Nanodrops from Molecular Dynamics}

\subsubsection{Numerical setup}

We perform Molecular Dynamics (MD) simulations on nanodrops, using the GROMACs software package [30]. We simulate binary systems in which two types of particles exist: fluid particles that can move around either in the gas or liquid phase, and solid particles which are frozen on an fcc lattice and constitute the solid substrate,Fig. 7.3(a). The simulations are done in the 


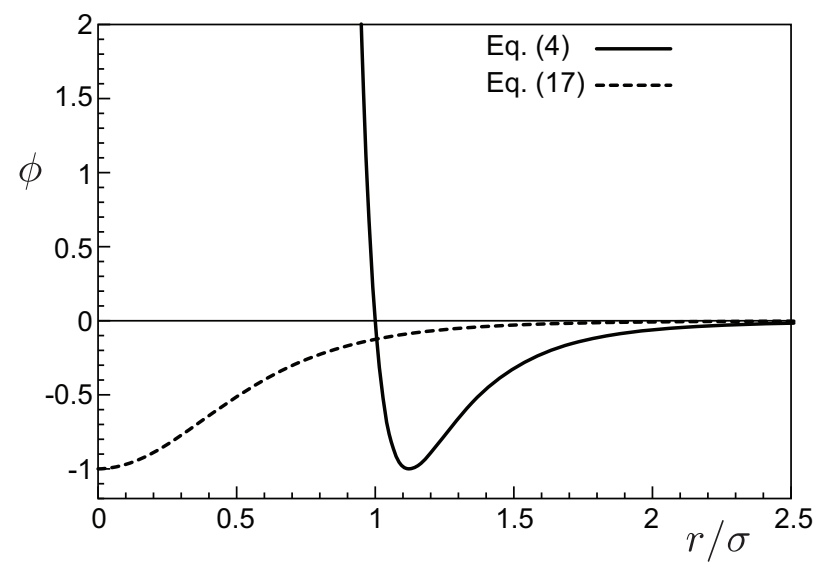

Figure 7.2: Interaction potentials $\phi(r)$ for the Lennard-Jones particles (7.4) (solid line) and the potential $\rho^{(1)} \rho^{(2)} g_{r}(r) \phi(r)$ used for the DFT-calculations (7.17) (dashed). Note that the DFT potential is regularized to account for vanishing $g_{r}(r)$ when $r \rightarrow 0$.

NVT-ensemble, where the temperature is held at $300 \mathrm{~K}$ using a thermostat, which is below the critical point for a Lennard-Jones fluid with the interaction strengths used. All particle interactions are defined by the LennardJones (LJ) potential:

$$
\phi_{i j}^{L J}(r)=4 \epsilon_{i j}\left[\left(\frac{\sigma_{i j}}{r}\right)^{12}-\left(\frac{\sigma_{i j}}{r}\right)^{6}\right],
$$

see also Fig. 7.2. Here, $\epsilon_{i j}$ is the interaction strength between particles $i$ and $j$ and $\sigma_{i j}$ the characteristic size of the molecules. This size is chosen the same for all interactions, $\sigma_{i j}=\sigma$. The potential function is truncated at a relatively large radius $\left(r_{c}=5 \sigma\right)$ where $\phi^{L J}$ is practically zero. The timestep is chosen at $d t=\sigma \sqrt{m / \epsilon_{l l}} / 200$, with $m$ the mass of the particles. The fluid particles are initially positioned on an fcc-lattice near the substrate, but are free to move around and relax towards an equilibrium droplet shape (Fig. 7.3). Periodic boundary conditions are present in all directions. To study the effect of line tension we consider two different systems. In the "3D" case the dimensions of the system are chosen large enough to ensure that the droplet does not interact with itself, resulting in an isolated droplet with the shape of a spherical cap. In the "2D" case the system size in the $x$-direction (parallel to the 
substrate) is only $15 \sigma$ leading to an infinitely long cylindrical-cap shaped droplet. The small length is required to suppress the Rayleigh-instability, which is only effective at wavelengths $\lambda>2 \pi R$. Now, as there is no contact line curvature, line tension has no effect.

The wettability of the substrate (and thus, the equilibrium contact angle) is tuned through the parameter ratio $\epsilon_{L S} / \epsilon_{L L}$, where the subscript indicator $S$ denotes the solid (fixed) particles and $L$ the liquid particles. A higher value for $\epsilon_{L S} / \epsilon_{L L}$ results in a large attraction of fluid particles to the substrate, and thus a more wetting substrate. A range of contact angles can be explored in this way, as shown in table I. The obtained contact angles and compare well to those found in previous studies [31, 32]. Depending on the size of the droplet, the effect of layering inside the liquid (Fig. 7.3) limits the range where reliable contact angle measurements can be performed. In practice, this limits the analysis to contact angles larger than approximately $70^{\circ}$.

\subsubsection{Cylindrical vs spherical caps}

Fig. 7.3(a) shows the shape of two nano-drops $\left(\theta_{Y}=65^{\circ}\right)$ with similar radii $R$ (as seen from the top), but with a different geometry. The cylindrical droplet on the left is formed in the quasi-2D system (several periods shown). The spherical cap shaped droplet on the right is simulated in a fully ${ }_{3} \mathrm{D}$ system. Fig. 7.3(b) shows the isodensity contours from the same droplets. One observes that these cross-sectional shapes are already very similar, indicating that line tension is indeed a weak effect, even for such nanodrops. We will now perform careful and precise contact angle measurements in order to quantify line tension.

\subsubsection{Measurement of contact angle}

To perform precise contact angle measurements we first compute the density field by averaging over time and over space (translational or rotational symmetry). During this averaging, we compensate for any center of mass motion of the droplet parallel to the substrate by moving the droplet such that the center of mass is stationary throughout the averaging procedure. When the droplet has reached its equilibrium state (Fig. $7 \cdot 3$ ), the density profiles are calculated by time-averaging over 1,000,000-10,000,000 time steps until the density field has converged. Using real world parameters for Argon as the fluid this would correspond to 2 - 20 nanoseconds. This leads to droplet shapes as shown in the bottom row of Fig. 7.3. The part of the droplet that 

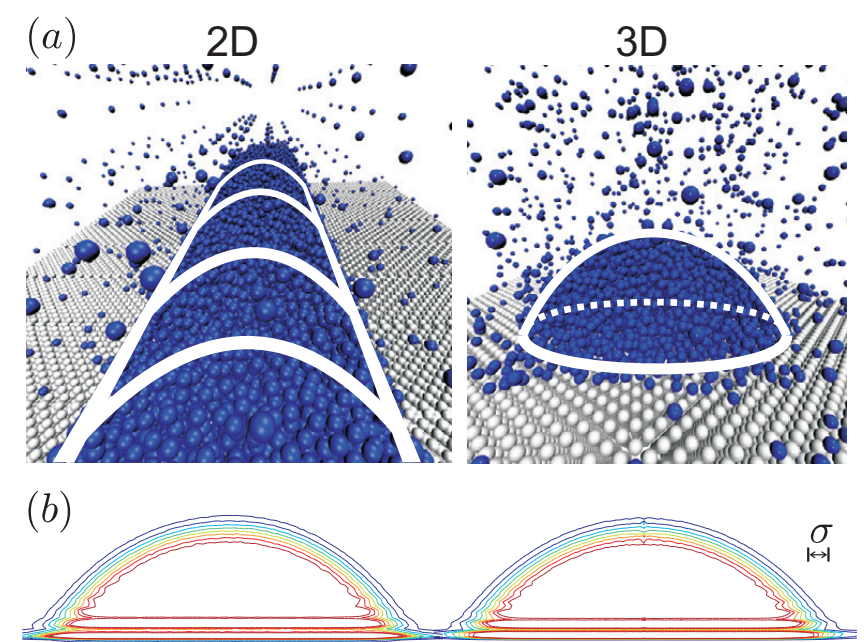

Figure 7·3: (a) Snapshots from Molecular Dynamics simulations of a 2D, cylindrical cap-shaped droplet (left), and a $3 \mathrm{D}$, spherical cap-shaped droplet (right). The light spheres represent the immobilized solid particles, forming the substrate to which the droplet attaches. The darker spheres represent the mobile fluid particles. The lines are a guide to the eye. Several periods of the $2 \mathrm{D}$ droplet are shown (periodic boundary conditions), causing the same particle to be printed multiple times. These drops were simulated using identical interaction parameters $\left(\frac{\epsilon_{S L}}{\epsilon_{L L}}=\frac{2}{3} \Rightarrow \theta_{Y} \approx 65^{\circ}\right)$, and differ in shape only because of the difference in the periodic boundary conditions. (b) Isodensity contours measured using statistical averaging from the droplets shown in the top row. The contact angle and overall shape of the two drops is almost identical, requiring a precise measurement to observe the effect of line tension. 


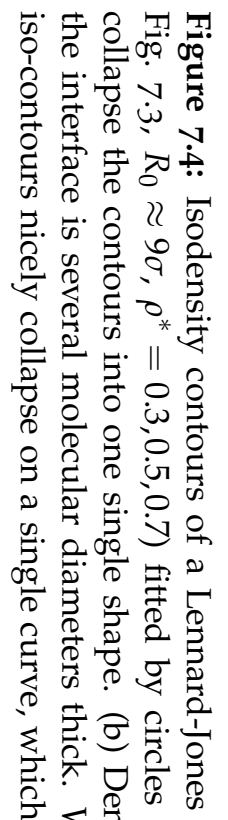

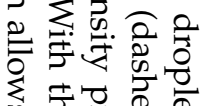

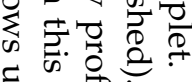

क

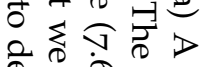

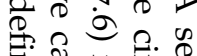
节寻总

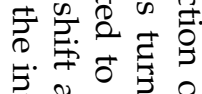

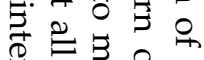

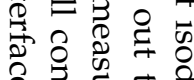
ठ. ఫे के के క.

막 $\stackrel{*}{=}$ 。 क. क्षे

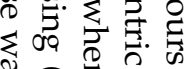
ঐণิ

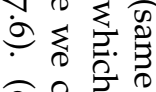

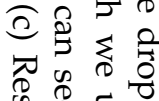
कृ के के 迅

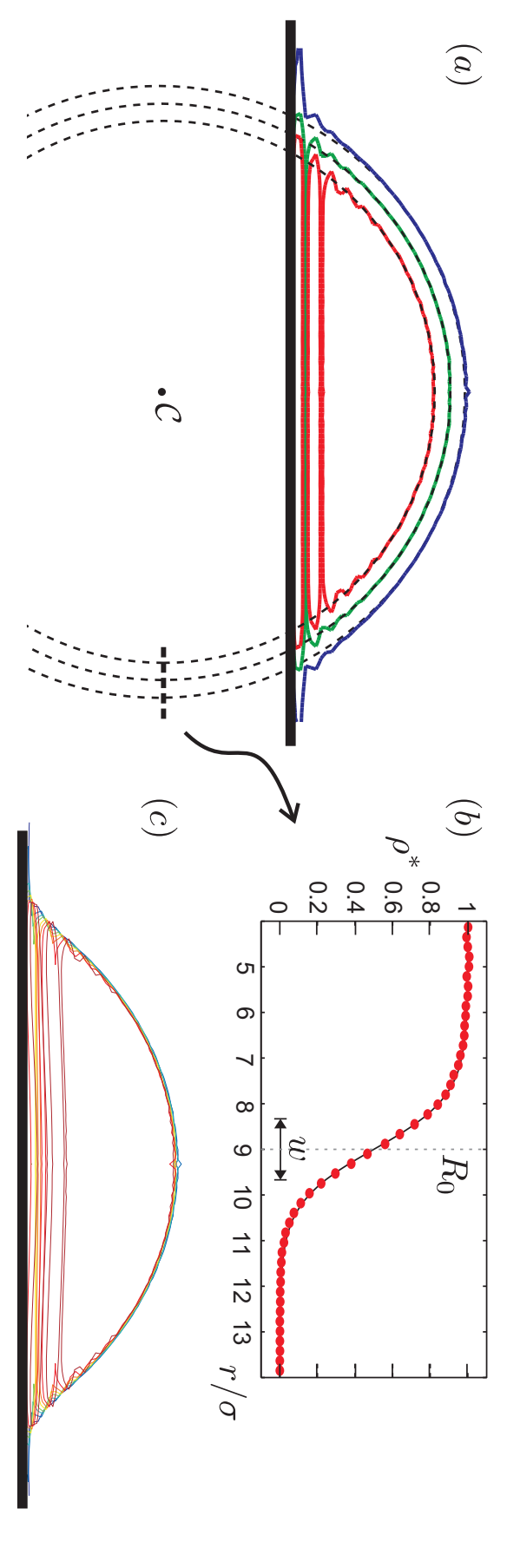


is close to the substrate is subject to layering [32]: the density oscillates as a function of height. To avoid interference from this effect, we ignore this part of the droplet when determining the contact angle: we perform a circular fit through the top part of the spherical cap, and extrapolate towards the substrate (which is defined to be $\sigma / 2$ above the top row of substrate atoms) to find $\theta$ and $R$. Fig. 7.4(a) shows these fits through some isodensity contours $\left(\rho^{*}=0.3,0.5,0.7\right)$.

This leads, however, to a new problem: which isodensity should one choose? As can readily be seen from Fig. 7.4(a) it turns out that the width of the interface cannot be neglected, and choosing different isodensity contours results in different values for the $\theta$ and $R$. To overcome this problem, we use the data from the cylindrical droplets to determine which isodensity contour to use. From a macroscopic perspective, the cylindrical caps are not affected by line tension $\left(\theta=\theta_{Y}\right)$. It turns out that this property is obeyed by the Gibbs dividing surface at $\rho^{*}=0.5$, where $\rho^{*}$ is a parametrized version of the local density given by:

$$
\rho^{*}(\vec{r})=\frac{\rho(\vec{r})-\rho_{V}}{\rho_{L}-\rho_{V}} .
$$

Here, $\rho_{L}$ and $\rho_{V}$ are the bulk densities of the liquid and vapour phase, respectively. We note that although line tension does not affect cylindrical droplets, other curvature effects (such as the Tolman-correction on $\gamma$ and the effect of the increased Laplace-pressure on $\gamma_{s l}$ ) do play a role. The baseline established by this methodology is therefore not based on 'pure' line tension, but rather an apparent line tension in which all these effects are combined. This is in line with previous experimental work, where this distinction could also not be made.

To improve statistics, we have also used the remaining isodensity contours. This can be done since the density profile across the interface is accurately fitted by (Fig. 7.4(b)):

$$
\rho^{*}=\frac{1}{2}\left(1+\tanh \left(\frac{R_{0}-r}{w}\right)\right),
$$

where $R_{0}$ is the point where the density is halfway between the liquid value and the vapour value $\left(\rho^{*}=0.5\right) . w$ is a fit parameter that defines the width of the liquid-vapour transition. Since the circular fits are concentric (they share a common center point, $\mathcal{C}$ in Fig. 7.4(a)) we can easily transform any isodensity contour to the reference contour. For this, we calculate the radial distance from the contour towards the reference contour using (7.6). The 
result of this transformation for the spherical droplet from Fig. $7 \cdot 3$ is shown in Fig. 7.4(c), where we see that the contour shapes indeed collapse and can now all be used to determine the contact angle. The spread of these values for different $\rho^{*}$ are used to determine the error of the measurements.

\subsubsection{Results: tension length}

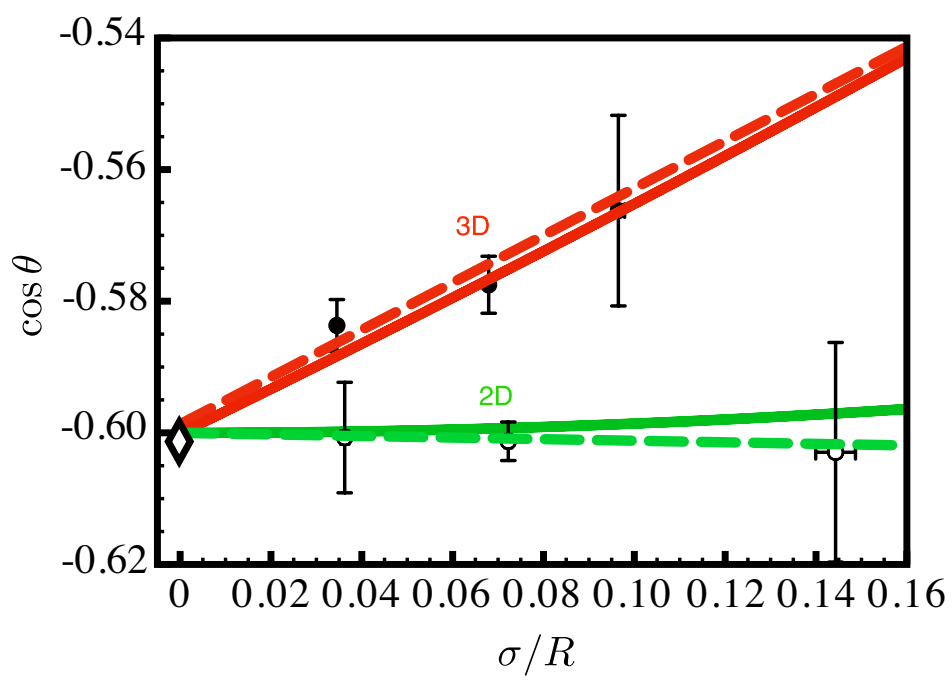

Figure 7.5: $\cos \theta$ vs $\sigma R^{-1}$ for cylindrical (open symbols) and spherical (filled symbols) drops for $\cos \theta_{Y}=-0.60\left(\approx 127^{\circ}\right)$. The dashed lines are linear fits through the data points, and the solid lines are the solutions obtained using DFT described in section 7.3. The top two (red) lines represent the $3 \mathrm{D}$-data, whereas the bottom two (green) lines the 2D-data. The diamond at $1 / R=0$ indicates Young's law, calculated independently by determining the surface tensions of the three interfaces: $\gamma, \gamma_{S L}$, $\gamma_{S V}$. The difference between the slopes of the $2 \mathrm{D}$ and ${ }_{3} \mathrm{D}$ fits quantifies the tension length $\ell$. Note that for this particular equilibrium contact angle, MD and DFT agree quantitatively on the tension length.

Figure 7.5 shows the relation between the contact angle defined as described above and the drop radius for different sized droplets. Young's angle $\theta_{Y}$ was independently calculated from independent measurements of the surface tensions [33] of planar interfaces, under the same simulation conditions as the droplet simulations. This is shown as the diamond symbol at $1 / R=0$. One can observe that the contact angle does not present any vari- 


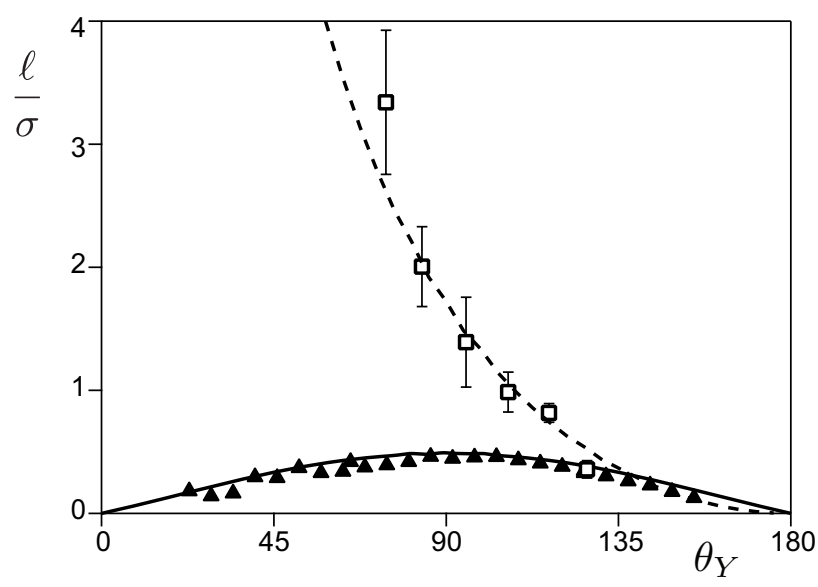

Figure 7.6: Tension length $\ell$ vs $\theta_{Y}$ for spherical drops. Square symbols are the molecular dynamics results, triangle symbols are the results from the self-consistent Density Functional Theory model discussed in section 7.3.1. These data points were acquired by measuring the contact angle for different drop sizes, meaning they represent an 'apparent' line tension. The solid and dashed lines also result from DFT, assuming a wedge-shaped geometry near the contact line: eqs. (7.25), (7.26), (7.27). For the self-consistent DFT data the characteristic lengths are determined analytically: $\zeta_{L L}=\zeta_{L S}=\pi \sigma / 4$. The resulting curve is the solid line. The characteristic lengths for the MD data are acquired by fitting: $\zeta_{L L}=3.5 \sigma, \zeta_{L S}=0$, represented by the dashed line.

\begin{tabular}{c|c|c|c}
$\epsilon_{L S} / \epsilon_{L L}$ & $\theta_{Y}$ & $\theta_{\text {DFT }}$ & $\ell(\sigma)$ \\
\hline \hline 0.33 & $129 \pm 1.0^{\circ}$ & $109^{\circ}$ & $0.36 \pm 0.02$ \\
\hline 0.40 & $117 \pm 1.2^{\circ}$ & $102^{\circ}$ & $0.82 \pm 0.03$ \\
\hline 0.47 & $106 \pm 1.3^{\circ}$ & $94^{\circ}$ & $0.99 \pm 0.09$ \\
\hline 0.53 & $95 \pm 1.8^{\circ}$ & $86^{\circ}$ & $1.39 \pm 0.20$ \\
\hline 0.60 & $84 \pm 1.5^{\circ}$ & $78^{\circ}$ & $2.01 \pm 0.18$ \\
\hline 0.67 & $74 \pm 1.8^{\circ}$ & $71^{\circ}$ & $3.34 \pm 0.32$
\end{tabular}

Table 7.1: MD results as shown in Fig. 7.6. The ratio $\epsilon_{L S} / \epsilon_{L L}$ was varied to obtain the tension length $\ell$ for different equilibrium contact angles $\theta_{Y} . \theta_{\mathrm{DFT}}$ is the contact angle resulting from the interaction ratio according to the DFT-model described in Sect. $7 \cdot 3$. 
ation with drop size in the cylindrical cap case, which is consistent with the macroscopic picture. By contrast, the spherical drops exhibit a decreasing contact angle for small radii (large $R^{-1}$ ), which according to (7.2) is consistent with a negative line tension $\tau$. A negative value of $\tau$ means that the contact line has the tendency to expand - a larger contact line length leads to a decrease in $\theta$ under the constant volume constraint. The solid line corresponds to the density functional theory in the sharp kink approximation that will be discussed below.

The difference between the slopes of the $2 \mathrm{D}$ - and $3 \mathrm{D}$-fits in Fig. $7 \cdot 5$ (dashed lines) is equal to the tension length, $\ell \equiv-\tau / \gamma$, which is defined to be positive for negative values of $\tau$ (see (7.3)). For this equilibrium contact angle $\left(\theta_{Y}=127^{\circ}\right)$ we find $\ell=0.36 \sigma$. Now, by varying the interaction ratio $\epsilon_{L S} / \epsilon_{L L}$ we measure $\ell$ for varying $\theta_{Y}$. The result is shown in table 7.1 and in Fig. 7.6 by the square symbols. Whatever $\theta_{Y}$, the tension length turns out to be positive (so $\tau$ is always negative) and very small - of the order of the atomic size $\sigma$. The tension length recovered from the MD simulations is a decreasing function of $\theta_{Y}$, indicating that the effect is stronger when the wedge formed by the liquid in the vicinity of the contact line is sharp. The other curves in Fig. 7.6 result from DFT, and will be discussed in the following sections.

\subsection{Origin of line tension effect}

In this section we study line tension in the framework of Density Functional Theory using the sharp kink approximation. Once more, the strategy is to determine the equilibrium shapes of $2 \mathrm{D}$ and ${ }_{3} \mathrm{D}$ drops and to compare their contact angles. Starting from the basic equations of DFT we first motivate the form of the free energy functional in Sec. 7.3.1. Some of the assumptions are directly tested using Molecular Dynamics simulations. We then derive the equilibrium condition for the capillary pressure (Sec. 7.3.2) and describe the numerical scheme that was used to solve the equilibrium shapes of the drops (Sec. 7.3.3). The numerical results are presented and interpreted in detail in Secs. 7.3.4 and 7.3.5.

\section{7-3.1 Density Functional Theory in the sharp kink approximation}

The primary idea of Density Functional Theory (DFT) is to express the grand potential $\Omega=U-T S-\mu N=F-\mu N$ as a functional of the particle density 
$\rho$ and to perform a functional minimisation for a given $\mu$ and T. For an ideal gas, the free energy functional is known explicitly

$$
F_{i d}[\rho]=k T \int \rho\left[\ln \left(\rho \Lambda^{3}\right)-1\right] d \vec{r},
$$

but this is not the case for general liquids. Let us denote $\phi\left(\vec{r}_{1}, \vec{r}_{2}\right)$ or $\phi(r)$ as the additive pair potential between particles at $\vec{r}_{1}$ and $\vec{r}_{2}$ with distance $r=\left|\vec{r}_{2}-\vec{r}_{1}\right|$. From a grand canonical averaging, one can show (see e.g. refs. $[34,35])$

$$
\frac{\delta \Omega}{\delta \phi\left(\vec{r}_{1}, \vec{r}_{2}\right)}=\frac{1}{2} \rho^{(2)}\left(\vec{r}_{1}, \vec{r}_{2}\right)=\frac{1}{2} \rho\left(\vec{r}_{1}\right) \rho\left(\vec{r}_{2}\right) g\left(\vec{r}_{1}, \vec{r}_{2}\right),
$$

where $\rho^{(2)}$ is the two-body density distribution function and $g$ the pair correlation function. This relation can be used to construct the free energy for non-ideal systems. Introducing a coupling parameter $\lambda$ in front of the interaction, the free energy can be constructed by integration as:

$$
\begin{array}{r}
F[\rho]=F_{i d}[\rho]+ \\
\frac{1}{2} \int_{0}^{1} d \lambda \int d \vec{r}_{1} \int d \vec{r}_{2} \rho\left(\vec{r}_{1}\right) \rho\left(\vec{r}_{2}\right) g_{\lambda}\left(\vec{r}_{1}, \vec{r}_{2}\right) \phi\left(\left|\vec{r}_{2}-\vec{r}_{1}\right|\right) .
\end{array}
$$

Here $g_{\lambda}$ is the pair correlation function in a system of same geometry and same volume, for which the interaction is $\lambda \phi(r)$.

Although exact, this expression cannot be used as it is, as $g_{\lambda}$ is not known. For a practical approximation of the energy functional, one can separate the thermodynamic non-ideality in contributions due to attractive and repulsive components of the intermolecular potential. As the repulsive forces have a very short range, their effect is mainly local. Using the Local Density Approximation, the repulsive contribution can be estimated from the Helmoltz energy density $f_{r}(\rho)$ in a uniform system of density $\rho$ at temperature $T$, composed of purely repulsive molecules. The attractive Van der Waals interactions, $\phi_{a t t}$, can then be treated as a perturbation, assuming that the pair correlation function remains mostly that of the purely repulsive reference system, $g_{r}\left(\vec{r}_{1}, \vec{r}_{2}\right)$. The free energy then reads:

$$
\begin{array}{r}
F[\rho]=\int f_{r}(\rho) d \vec{r}+ \\
\frac{1}{2} \int d \vec{r}_{1} \int d \vec{r}_{2} \rho\left(\vec{r}_{1}\right) \rho\left(\vec{r}_{2}\right) g_{r}\left(\vec{r}_{1}, \vec{r}_{2}\right) \phi_{a t t}\left(\left|\vec{r}_{2}-\vec{r}_{1}\right|\right) .
\end{array}
$$




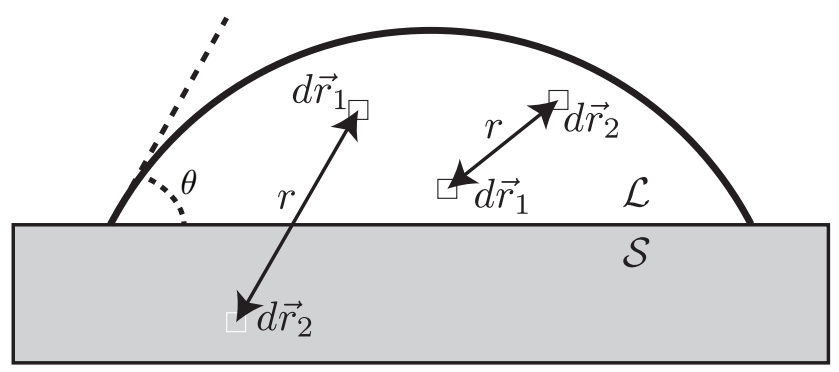

Figure 7.7: Schematic representation of the integration variables and domains from (7.11). Both the liquid-solid (left) and the liquid-liquid (right) interactions are integrated over their respective volumes (the liquid cap $\mathcal{L}$ and the solid substrate $\mathcal{S}$ ) to obtain the total free energy $F$.

To end up with a numerically tractable scheme, we make a final approximation that the density profile across the interface is mostly independent of the geometry. Defining the position of the interface e.g. by the iso-density $\rho^{*}=1 / 2$, the integrals in (7.10) can be approximated by assuming that the density is uniform in both phases [6].This so-called 'sharp kink approximation' neglects the thickness of the diffuse interface. Thermal effects are implicitly taken into account, since $f_{r}, g_{r}$ and the liquid density depend on temperature. In this approximation, the free energy becomes an explicit functional of the shape of liquid, solid, and vapor domains. Since the vapor density is neglible with respect to that of the solid and liquid, we find

$$
\begin{aligned}
F & =f_{r}\left(\rho_{L}\right) \int_{\mathcal{L}} d \vec{r}+f_{r}\left(\rho_{S}\right) \int_{\mathcal{S}} d \vec{r} \\
& +\frac{1}{2} \rho_{L}^{2} \int_{\mathcal{L}} d \vec{r}_{1} \int_{\mathcal{L}} d \vec{r}_{2} g_{r}\left(\left|\vec{r}_{2}-\vec{r}_{1}\right|\right) \phi_{L L}\left(\left|\vec{r}_{2}-\vec{r}_{1}\right|\right) \\
& +\rho_{L} \rho_{S} \int_{\mathcal{L}} d \vec{r}_{1} \int_{\mathcal{S}} d \vec{r}_{2} g_{r}\left(\left|\vec{r}_{2}-\vec{r}_{1}\right|\right) \phi_{L S}\left(\left|\vec{r}_{2}-\vec{r}_{1}\right|\right),
\end{aligned}
$$

where $\mathcal{L}$ and $\mathcal{S}$ are the liquid and solid domains respectively, see also Fig. 7.7. $\phi_{L L}$ and $\phi_{L S}$ denote the attractive parts of the respective interactions. The drop shapes are found by minimizing this free energy with respect to the shape of the liquid domain $\mathcal{L}$.

Before proceeding, it is instructive to discuss the approximations underlying (7.11) in the light of the Molecular Dynamics simulations of the 
Lennard-Jones droplets. First, the assumption that the pair-correlation function is homogeneous in space ignores the layering near the solid wall (cf. Fig. 7.3). This can induce significant corrections to the estimated free energy. Second, the Local Density Approximation of the short-range repulsive forces gives rise to isotropic repulsive interactions, while the attractive interactions will become anisotropic in the vicinity of an interface. If this is indeed the case, the surface tension (and line tension) effects mostly result from the attractive component of the interaction. We test the validity of this hypothesis in the Molecular Dynamics simulations by measuring the anisotropy of the stress tensor in the vicinity of the liquid-vapour interface. We define a cumulative stress-tensor $\sigma^{\alpha \alpha}\left(R^{*}\right)$ that incorporates only the interactions with a bond length smaller than $R^{*}$ :

$$
\overline{\bar{\sigma}}^{\alpha \alpha}\left(R^{*}\right)=\sum_{i} m_{i} v_{i}^{\alpha} v_{i}^{\alpha}-\sum_{j \neq i} \sum_{\left|r_{i j}\right|<R^{*}} f_{i j}^{\alpha} r_{i j}^{\alpha} .
$$

The true stress in the system is recovered when $R^{*}=\infty$, for which all interactions are taken into account. Here, $m_{i}, v_{i}$ are the mass and velocity of particle $i$, respectively, and $f_{i j}$ and $r_{i j}$ are the force and displacement vector between particles $i$ and $j$. With this, we quantify the anisotropy from the difference between the stress components tangential $(T)$ and normal $(N)$ to the interface, as

$$
A\left(R^{*}\right)=\frac{\overline{\bar{\sigma}}^{T T}\left(R^{*}\right)-\overline{\bar{\sigma}}^{N N}\left(R^{*}\right)}{\overline{\bar{\sigma}}^{T T}(\infty)+\overline{\bar{\sigma}}^{N N}(\infty)} .
$$

Figure 7.8 shows the anisotropy $A$ as a function of $R^{*}$. The dashed line indicates the transition from the repulsive $\left(r<2^{1 / 6} \sigma\right)$ to the attractive domain $\left(r>2^{1 / 6} \sigma\right)$. The figure clearly shows that the majority of the anisotropy in the liquid-vapour interface is due to the attractive interaction, while the repulsive interaction accounts for about $20 \%$ of the anisotropy. This indeed justifies a local density approximation for the repulsion, although one can expect quantitative differences with Molecular Dynamics.

\subsubsection{Capillary pressure}

The equilibrium shape of liquid drops can be obtained by minimizing the free energy $F$ at constant volume $V$. This can be done by variation of (7.11) with respect to the drop shape $\mathcal{L}$ under the constraint of constant volume. The resulting equilibrium condition is a constant potential energy density 


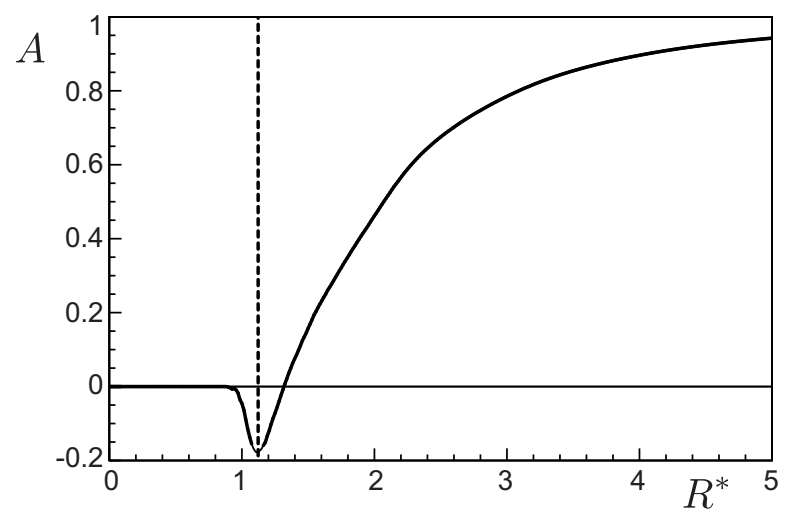

Figure 7.8: Stress anisotropy $A$ for bond lengths smaller than $R^{*}$, see (7.13). The measurement was done in a slab of height $\sigma / 3$ within the liquid-vapour interface in a molecular dynamics simulation. The dashed line indicates the minimum of the Lennard-Jones potential at $R^{*}=2^{1 / 6} \sigma$ and marks the separation between the attractive and repulsive bonds. The majority of the anisotropy comes from attraction.

$\Pi$ along the free surface $[6,36,37]$. This potential can be interpreted as the capillary pressure and can be decomposed into a liquid-liquid and a solidliquid contribution, as $\Pi=\Pi_{L L}+\Pi_{L S}$. The former can be written as

$$
\Pi_{L L}(\vec{r})=-\Pi_{L L}^{\circ}+\rho_{L} \int_{\mathcal{L}} d \vec{r}^{\prime} g_{r}\left(\left|\vec{r}^{\prime}-\vec{r}\right|\right) \phi_{L L}\left(\left|\vec{r}^{\prime}-\vec{r}\right|\right),
$$

where we subtracted $\Pi_{L L}^{\circ}$, the interaction due to a semi-infinite volume of liquid. The solid-liquid contribution follows from the interaction due to the semi-infinite volume of solid

$$
\Pi_{L S}(\vec{r})=\rho_{S} \int_{\mathcal{S}} d \vec{r}^{\prime} g_{r}\left(\left|\vec{r}^{\prime}-\vec{r}\right|\right) \phi_{L S}\left(\left|\vec{r}^{\prime}-\vec{r}\right|\right) .
$$

The equilibrium condition is thus that

$$
\Pi\left(\vec{r}^{*}\right)=\Pi_{L L}\left(\vec{r}^{*}\right)+\Pi_{L S}\left(\vec{r}^{*}\right)=\text { constant, }
$$

where $\vec{r}^{*}$ denotes an arbitrary position at the liquid-vapor interface.

Note that the capillary pressure $\Pi$ depends on the shape of the liquid, through the domain of integrations, and thus reflects the effect of the interface geometry on the free energy. It implicitly contains the Laplace pressure, which is the capillary pressure associated to a macroscopic curvature of the interface, and the disjoining pressure, which is the capillary pressure in the case of a microscopic film. 


\subsubsection{Shape relaxation}

We compute droplet shapes for a pair interaction consistent with the longrange van der Waals interaction used in the Molecular Dynamics simulations (see Fig. 7.2):

$$
\rho_{i} \rho_{j} g_{r}(r) \phi_{i j}(r)=\frac{-c_{i j}}{\left(\sigma^{2}+r^{2}\right)^{3}},
$$

where $c_{i j}$ represents the strength of the interaction between molecule $i$ and $j$. Comparing to the van der Waals interaction of (7.4), one finds $c_{i j}=$ $4 \rho_{i} \rho_{j} \sigma^{6} \epsilon_{i j}$. For mathematical convenience we have chosen a simple regularization around $r=\sigma$, which represents the effective size of the short-range repulsion. Let us note that the potential of equation (17) does not lead to the formation of a precursor film. Namely, the corresponding energy per unit surface for a flat film is a monotonic function of the thickness $h$, with a prefactor depending on the spreading parameter. For partial wetting, the system tends to zero thickness rather than to a precursor film of finite thickness. We have tested that other similar choices like $g(r)=0$ for $r<\sigma$ and $g(r)=1$ for $r>\sigma$ leads to quantitatively similar results (see ref. [37]). The surface tensions corresponding to (7.17) can be computed as

$$
\gamma=\pi c_{l l} / 8 \sigma^{2}
$$

and

$$
\gamma+\gamma_{s v}-\gamma_{s l}=\pi c_{l s} / 4 \sigma^{2}
$$

By choosing identical functional forms for both interactions, one simply has [34]

$$
\cos \theta_{Y}=\cos \theta_{D F T} \equiv 2 \frac{c_{l s}}{c_{l l}}-1
$$

Similar to the Molecular Dynamics simulations, we compute the equilibrium shapes of nanodrops in both the $2 \mathrm{D}$ configuration (cylindrical caps) and $3 \mathrm{D}$ configuration (spherical caps). The drop shapes are parameterized by $r(\alpha)$ as shown in Fig. 7.9 - polar coordinates are used to allow contact angles larger than $\pi / 2$. We numerically determine the equilibrium shape of the drop by an iterative algorithm that tends to a constant $\Pi(\alpha)$ along the interface. The initial shape is taken as a spherical cap with $\theta_{Y}$ according to (7.20). This is shown in Fig. 7.9 by dashed lines. The corresponding potential $\Pi(\alpha)$ is uniform except within a few molecular scales from the contact line, 

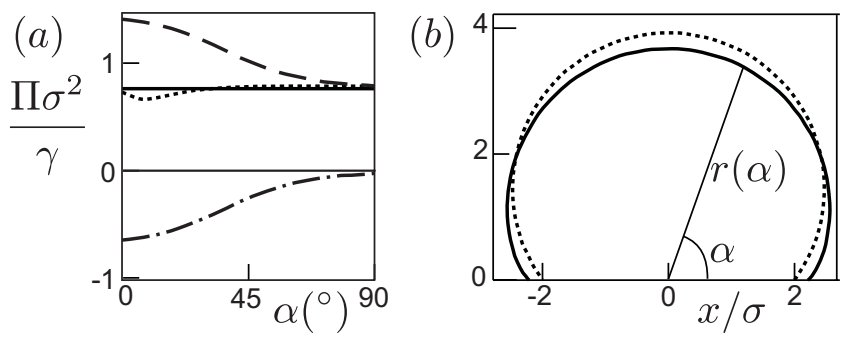

Figure 7.9: Typical result of the DFT model in the sharp kink approximation. (a) Surface potentials of an axisymmetric drop with $\theta_{Y}=127^{\circ}$, a measured angle $\theta=$ $116^{\circ}$ and radius $R \approx 2 \sigma . \alpha=0$ at the contact line. The dashed line (top) represents $\tilde{\Pi}_{l l}$, the bottom line represents $\Pi_{s l}$, and the lines in the middle indicate the sum of the two. Here, the dashed and solid lines indicate the potential energy density of the droplet in its initial shape, and its equilibrium (final) shape, respectively. (b) Initial and final drop profiles shown by the dashed and solid lines, respectively. The initial profile is a spherical cap shaped drop with $\theta=\theta_{Y}=127^{\circ}$.

where the influence of the solid plays a role. We iteratively construct drop shapes $r^{t}(\alpha)$ according to $r^{t+1}(\alpha)=r^{t}(\alpha)+\lambda^{t}\left(\pi^{t}(\alpha)-\left\langle\pi^{t}\right\rangle_{\alpha}\right)$, while keeping the volume constant. Here, $\pi^{t}(\alpha)$ is the capillary pressure at angle $\alpha$ during iteration $t .\left\langle\pi^{t}\right\rangle_{\alpha}$ is the space-averaged potential at the interface during iteration $t$. The parameter $\lambda^{t}$ is selected such that the variance of the potential is minimised at each step. After a few hundred steps, the shape converges and yields $\Pi(\alpha)$ that indeed is constant within numerical precision. Note that for the potential studied here, no precursor film is formed.

The shape $r(\alpha)$ and the capillary pressure $\Pi(\alpha)$ of a small drop are plotted as solid lines in Fig. 7.9. Away from the contact line the drop is a spherical cap, but a significant deviation can be observed near the contact line. The drop has spread with respect to the initial shape, resulting into a lower contact angle than $\theta_{Y}$. Once more, this is consistent with a negative value of the line tension $\tau$. Far from the contact line, the capillary pressure is dominated by the $\Pi_{l l}$ term. The corresponding value is simply the expected Laplace pressure $2 \gamma / \tilde{R}$, where $\tilde{R}$ is the radius of curvature the drop.

\subsubsection{Results}

In Fig. 7.10 we compare the contact angles of $3 \mathrm{D}$ drops (squares) and $2 \mathrm{D}$ drops (triangles), as a function of inverse drop radius $1 / R$. In both cases 


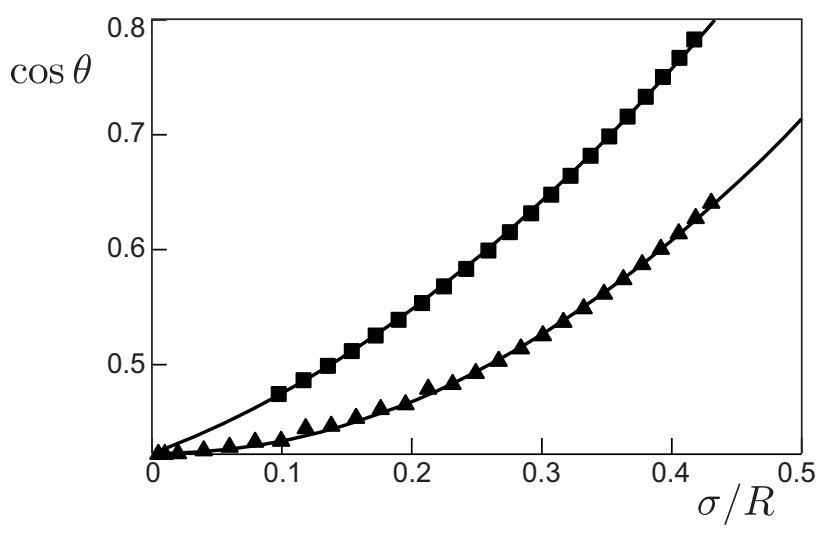

Figure 7.10: Cosine of the equilibrium contact angle against $1 / R$, for $2 \mathrm{D}$ drops (triangles) and ${ }_{3} \mathrm{D}$ drops (squares). The corresponding Young's angle is $\theta_{Y}=65^{\circ}$. The slope of the curve near $1 / R=0$ can be attributed to line tension for the ${ }_{3} \mathrm{D}$ drops, while it is zero for $2 \mathrm{D}$ drops. Note that both curves exhibit a significant $1 / R^{2}$ contribution for smaller drop sizes. This contribution was not recovered from the molecular dynamics simulations, since the radius of the droplets was not small enough: $R>7 \sigma$. Smaller droplets would not allow for spherical cap fitting because the droplet size becomes similar to the particle size.

the interactions were identical, corresponding to $\theta_{Y}=65^{\circ}$. For large drops there is indeed a difference that can be attributed to line tension: the slope at $1 / R \rightarrow 0$ is finite for $3 \mathrm{D}$ drops while it vanishes in the $2 \mathrm{D}$ case. Interestingly, however, there remains a $1 / R^{2}$ contribution for both types of drops. The two data sets are accurately fitted by parabola, with equal prefactors for the quadratic term. This suggests that the effect of line tension in (7.2) can be seen as the leading order contribution of an expansion in $\sigma / R$, and is only valid for relatively large drops. In particular, (7.2) must break down when $\cos \theta \approx 1$. This is illustrated by Fig. 7.11 showing a saturation of the contact angle to $\theta \approx 0$ for very small drops. This effect is of course most pronounced for drops that already have a small Young's angle $\theta_{Y}$. For such small drops, the range over which one observes a $1 / R$ behaviour is very small and the main size effect is to induce a wetting transition.

We are now in the position to make a comparison of the DFT model with the molecular dynamics simulations presented in Sec. 7.2. The solid line in Fig. 7.5 represents the contact angles for $3 \mathrm{D}$ drops of varying sizes as obtained from the numerical DFT calculation. We took the same Young's 


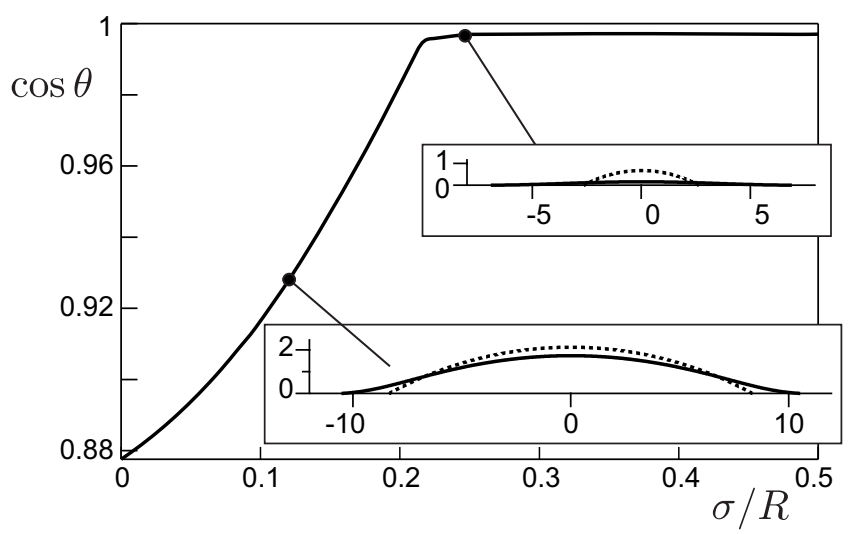

Figure 7.11: Cosine of the equilibrium contact angle against $1 / R$, for a $3 \mathrm{D}$ drop $\theta_{Y}=28^{\circ}$ as shown in the insets for two different sizes $R$. At sufficiently small radius we observe a saturation of $\cos (\theta)=1$, approaching a perfectly wetting drop (top inset).

angle as obtained in the molecular dynamics simulations, i.e. $\theta_{Y}=127^{\circ}$. The trends of DFT and molecular dynamics are very similar, clearly showing a decrease in contact angle for decreasing drop radius. For both cases line tension is thus negative and has a similar magnitude in units of $\sigma$.

Finally, we determined the tension length $\ell$ from the slope near $1 / R \rightarrow 0$, for a broad range of $\theta_{Y}$. The results are reported as triangles in Fig. 7.6. The value of $\ell$ vanishes both for 0 and $180^{\circ}$ and presents a maximum around $90^{\circ}$. Beside the sign and the order of magnitude, the behaviour is thus qualitatively different from the molecular dynamics results. This difference is most pronounced at small $\theta_{Y}$ (Fig. 7.6).

\subsubsection{Geometric interpretation of line tension}

Within our DFT model, the dependence of $\ell$ on contact angle $\theta_{Y}$ can be accurately described from a geometric argument (solid line in Fig. 7.6). We separate the free energy (7.11) in volumic, surfacic and linear contributions, as $F=P V+\sum_{i} \gamma_{i} S_{i}+\tau L$. By assuming the liquid domain to be wedgeshaped, it is indeed possible to explicitly separate the domains of integration 


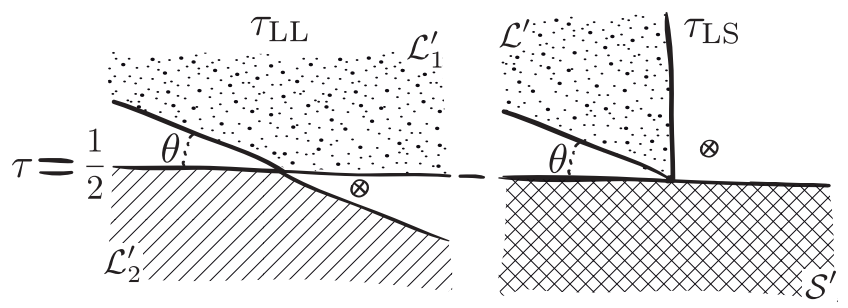

Figure 7.12: Integration domains of the free energy (7.11) that contribute to line tension due to liquid-liquid interactions $\tau_{L L}$ (left) and solid-liquid interactions $\tau_{L S}$ (right). Assuming the contact line region to be perfectly wedge-shaped the total free energy $F$ minus the volumic and surfacic contributions results in a residual energy, which can be attributed to line tension. We show in the Appendix how the line tension contribution can be isolated from the liquid-liquid and solid-liquid interactions. Then, by calculating the free energy associated with these integration domains (Eq. (7.21)) one directly finds the line tension due to liquid-liquid interactions and solid-liquid interactions: eqs. (7.25), (7.26), and (7.27).

in (7.11) in bulk, surface, and line contributions:

$$
\begin{aligned}
F & =P V+\sum_{i} \gamma_{i} S_{i} \\
& +\frac{1}{2} \rho_{L}^{2} \int_{\mathcal{L}_{1}^{\prime}} d \vec{r}_{1} \int_{\mathcal{L}_{2}^{\prime}} d \vec{r}_{2} g_{r}\left(\left|\vec{r}_{2}-\vec{r}_{1}\right|\right) \phi_{L L}\left(\left|\vec{r}_{2}-\vec{r}_{1}\right|\right) \\
& +\rho_{L} \rho_{S} \int_{\mathcal{L}^{\prime}} d \vec{r}_{1} \int_{\mathcal{S}^{\prime}} d \vec{r}_{2} g_{r}\left(\left|\vec{r}_{2}-\vec{r}_{1}\right|\right) \phi_{L S}\left(\left|\vec{r}_{2}-\vec{r}_{1}\right|\right) .
\end{aligned}
$$

Here the integration domains $\mathcal{L}_{1}^{\prime}, \mathcal{L}_{2}^{\prime}, \mathcal{L}^{\prime}$, and $\mathcal{S}^{\prime}$ are those represented in Fig. 7.12 and the Appendix. Note that such a decomposition is uniquely defined in the sharp-kink approximation, while this is no longer the case for inhomogeneous density profiles.

From (7.21) one sees directly that line tension has two contributions, due to liquid-liquid interactions $\tau_{L L}$, and due to liquid-solid interactions $\tau_{L S}$. These can be computed as follows. The integration domains $\mathcal{L}_{1}^{\prime}, \mathcal{L}_{2}^{\prime}, \mathcal{L}^{\prime}$, and $\mathcal{S}^{\prime}$ are bordered by straight lines passing through the contact line so that they do not present a characteristic scale. Therefore, both $\tau_{L L}$ and $\tau_{L S}$ can be written as products of a characteristic length (that does not depend on $\theta$ ), and a function of $\theta$ (that does not depend on the potentials $\phi_{L L}$ and $\phi_{L S}$ ). It turns out that the lengths can be expressed in terms of the liquid-liquid 
and solid-liquid disjoining pressures $\Pi_{L L}^{\mathrm{disj}}(h)$ and $\Pi_{L S}^{\mathrm{disj}}(h)$. The disjoining pressure is the energy per unit liquid volume at a distance $h$ from a flat semiinfinite zone of liquid or solid (see the integration domain $\mathcal{S}^{\prime}$ in Fig. 7.12). The surface tensions, already computed in $(7 \cdot 18,7.19)$, can be expressed as the integrals of these quantities:

$$
\begin{array}{r}
\int \Pi_{L L}^{\mathrm{disj}}(h) d h=2 \gamma \\
\int \Pi_{L S}^{\mathrm{disj}}(h) d h=\gamma+\gamma_{s v}-\gamma_{s l}=\gamma\left(1+\cos \theta_{\Upsilon}\right)
\end{array}
$$

The characteristic lengths $\zeta_{L L}$ and $\zeta_{L S}$ that appear in the calculation of the line tension turn out to be the first moment of the disjoining pressure:

$$
\zeta_{L L}=\frac{\int z \Pi_{L L}^{\mathrm{disj}}(z) d z}{\int \Pi_{L L}^{\mathrm{disj}}(z) d z} \quad \text { and } \quad \zeta_{L S}=\frac{\int z \Pi_{L S}^{\mathrm{disj}}(z) d z}{\int \Pi_{L S}^{\mathrm{disj}}(z) d z}
$$

Following the interpretation of surface tension as a force per unit length, $\zeta_{L L}$ and $\zeta_{L S}$ are the "moment arms" of these forces. Within our DFT model, the liquid-liquid and solid-liquid potentials have the same shape so that these two lengths are equal, $\zeta_{L S}=\zeta_{L L}=\pi \sigma / 4$.

The line tension $\tau_{L S}$ follows as:

$$
\tau_{L S}=\zeta_{L S} \gamma \frac{\left(1+\cos \theta_{Y}\right)}{\tan \theta} .
$$

This contribution is positive for $0<\theta<\pi / 2$ and changes sign at $\theta=\pi / 2$. The prefactor $\left(1+\cos \theta_{Y}\right)$ is not of geometric origin, but stems from the strength of the liquid-solid interaction $c_{L S}$. A similar result for $\tau_{L S}$ was previously obtained in ref. [38], but this work omitted the contribution due to liquid-liquid interactions, $\tau_{L L}$, which is crucial to describe our numerical DFT results. The contribution due to liquid-liquid interactions is negative for all angles. In the limit of small angles, $\tau_{L L}$ diverges as

$$
\tau_{L L} \equiv-\zeta_{L L} \gamma \frac{2}{\tan \theta} .
$$

As the angle $\theta$ tends to $\pi, \tau_{L L}$ vanishes as

$$
\tau_{L L}=-\frac{2}{3 \pi} \zeta_{L L} \gamma(\pi-\theta)^{2}
$$

In between, we have determined the ratio $\tau_{L L} / \zeta_{L L}$ by numerical integration. 
Adding the two contributions $\tau_{L L}$ and $\tau_{L S}$, we obtain the solid line in Fig. 7.6, which indeed closely follows the full numerical simulations obtained from the spherical cap measurements. Note that both $\tau_{L L}$ and $\tau_{L S}$ scale as $1 / \theta$ for small angles, but the diverging contributions balance exactly. This is a consequence of having identical values for the moment arms, i.e. $\zeta_{L L}=\zeta_{L S}$, resulting in a vanishing line tension for small $\theta$. Of course, this will not be the case in general, where we expect one of the contributions to dominate.

\subsection{Discussion}

We theoretically investigated the effect of line tension by studying the contact angles of Lennard-Jones droplets of varying sizes. The equilibrium shapes of nanodrops were determined using two methods: Molecular Dynamics (MD) and Density Functional Theory (DFT). For $3 \mathrm{D}$ drops we found a size-dependent contact angle consistent with (7.2), while the contact angle was nearly constant for $2 \mathrm{D}$ drops. DFT in the employed approximation does not fully reproduce the MD simulations, but it does capture the main physics. In particular, DFT gives the correct (negative) sign and order of magnitude of $\tau$, and also captures the dependence on wettability for large contact angles. Obiously, the exact numerical values resulting from the DFT calculation depend on our specific choice of the potential (7.17). Note however, that both the recovered trend and the orders of magnitude for $\ell$ are a general result, independent of the specific choice of the potential. The only exception is the limit of the wetting transition, $\theta \rightarrow 0$, which is known to depend on details of the interaction $[8,12,13]$.

In addition, we identified a simple geometric interpretation of line tension. Molecules inside a liquid wedge interact with a larger number of surrounding molecules than estimated from surface tension, which is based on an infinite half space of liquid. Hence the negative sign of line tension. The wedge shape of the liquid is indeed a good approximation of the liquid geometry for large contact angles and yields a very accurate prediction for $\tau$ in the DFT case. This is remarkable, since these DFT-measurements did not discriminate between line tension and other curvature effects, suggesting that line tension is the dominant mechanism for the size dependence of the contact angle. Once more, the behavior for small contact angles is sensitive to details of the interaction: it depends on the "moment arm" of the surface tensions, characterized by the length scales $\zeta_{L L}$ and $\zeta_{L S}$. We specu- 
late that the layering effect near the substrate in MD substantially reduces the moment arm $\zeta_{L S}$ for the liquid-solid interaction. This would explain the discrepancy with DFT. Indeed, the MD data can be described by the wedgeapproximation of $\tau_{L L}$ by fitting the moment arms to $\zeta_{L L}=3.5 \sigma$ and $\zeta_{L S}=0$. It would be interesting to further investigate this matter.

Although we were able to observe the variation of contact angle with drop size, the effect is only noticeable for very small, nano-scale drops. Taking $\sigma=0.34 \mathrm{~nm}$ and $\gamma=0.017 \mathrm{~J} / \mathrm{m}^{2}$, our results correspond to line tension in the range $\tau=10^{-12}-10^{-11} \mathrm{~J} / \mathrm{m}$ (depending on the wettability). This is consistent with theoretical predictions as well as with recent experiments [26]. Note, however, that much larger experimental values for $\tau$ have also been reported [16-18]. Resolving this issue is particularly important for surface nanobubbles $[22,25,39,40]$, typically $100 \mathrm{~nm}$ wide, whose stability was suggested to rely on an effective line tension [41].

\section{References}

[1] J. W. Gibbs, The Collected Works of J. Willard Gibbs (Yale University Press, London, 1957), p. 288.

[2] L. Guzzardi, R. Rosso, and E. G. Virga, Residual stability of sessile droplets with negative line tension, Phys. Rev. E 73, 021602 (2006).

[3] L. Schimmele and S. Dietrich, Line tension and the shape of nanodroplets, Eur. Phys. J. E 30, 427 (2009).

[4] L. Schimmele, M. Napiorkowski, and S. Dietrich, Conceptual aspects of line tensions, J. Chem. Phys. 127, 164715 (2007).

[5] B. Pethica, Contact-Angle Equilibrium, J. Colloid Interface Sci. 62, 567 (1977).

[6] T. Getta and S. Dietrich, Line tension between fluid phases and a substrate, Phys. Rev. E 57, 655 (1998).

[7] C. Bauer and S. Dietrich, Quantitative study of laterally inhomogeneous wetting films, Eur. Phys. J. B 10, 767 (1999).

[8] H. Dobbs and J. Indekeu, Line tension at wetting - interface displacement model beyond the gradient-squared approximation, Physica A 201, 457 (1993). 
[9] N. Churaev, V. Starov, and B. Derjaguin, The shape of the transition zone between a thin-film and bulk liquid and the line tension, J. Colloid Interface Sci. 89, 16 (1982).

[10] P.-G. De Gennes, Wetting - Statics and Dynamics, Rev. Mod. Phys. 57, 827 (1985).

[11] A. Amirfazli and A. W. Neumann, Status of the three-phase line tension: a review, Advances in Colloid and Interface Science 110, 121 (2004).

[12] J. Indekeu, Line Tension near the Wetting Transition - Results from an Interface Displacement Model, Physica A 183, 439 (1992).

[13] J. O. Indekeu, Line tension at wetting, Int. J. Mod. Phys. B 8, 309 (1994).

[14] J. Drelich and J. Miller, The Effect of Solid Surface Heterogeneity and Roughness on the Contact Angle/Drop (Bubble) Size Relationship, J. Colloid Interface Sci. 164, 252 (1994).

[15] J. Drelich and J. Miller, The line/pseudo-line tension in three-phase systems, Particul. Sci. Technol. 10, I (1992).

[16] J. Gaydos and A. W. Neumann, The Dependence of Contact Angles on Drop Size and Line Tension, J Colloid Interface Sci. 120, 76 (1987).

[17] D. Li and A. Neumann, Determination of line tension from the drop size dependence of contact angles, Colloids Surfaces 43, 195 (1990).

[18] R. Vera-Graziano, S. Muhl, and F. Rivera-Torres, The effect of illumination on contact angles of pure water on crystalline silicon, J. Colloid Interface Sci. 170, 591 (1995).

[19] J. Drelich, The significance and magnitude of the line tension in three-phase (solid-liquid-fluid) systems, Colloid Surf. A-Physicochem. Eng. Asp. 116, 43 (1996).

[20] A. Checco, P. Guenoun, and J. Daillant, Nonlinear Dependence of the Contact Angle of Nanodroplets on Contact Line Curvature, Phys. Rev. Lett. 91, 186101 (2003).

[21] D. Li, F. Lin, and A. Neumann, Effect of corrugations of the 3-phase line on the drop size dependence of contact angles, J. Colloid Interface Sci. 142, 224 (1991). 
[22] B. M. Borkent, S. de Beer, F. Mugele, and D. Lohse, On the Shape of Surface Nanobubbles, Langmuir 26, 260 (2010).

[23] J. Yang, J. Duan, D. Fornasiero, and J. Ralston, Very Small Bubble Formation at the Solid-Water Interface, J. Phys. Chem. B 107, 6139 (2003).

[24] N. Kameda, N. Sogoshi, and S. Nakabayashi, Nitrogen nanobubbles and butane nanodroplets at Si(100), Surface Science 602, 1579 (2008).

[25] N. Kameda and S. Nakabayashi, Size-induced sign inversion of line tension in nanobubbles at a solid/liquid interface, Chem. Phys. Lett. 461, 122 (2008).

[26] T. Pompe and S. Herminghaus, Three-phase contact line energetics from nanoscale liquid surface topographies, Phys. Rev. Lett. 85, 1930 (2000).

[27] Z. Zorin, D. Platikanov, and T. Kolarov, The transition region between aqueous wetting films on quartz and the adjacent meniscus, Colloids Surfaces 22, 147 (1987).

[28] J. D. Halverson, C. Maldarelli, A. Couzis, and J. Koplik, Atomistic simulations of the wetting behavior of nanodroplets of water on homogeneous and phase separated self-assembled monolayers, Soft Matter 6, 1297 (2010).

[29] T. Ingebrigtsen and S. Toxvaerd, Contact Angles of Lennard-Jones Liquids and Droplets on Planar Surfaces, J. Phys. Chem. C 111, 8518 (2007).

[30] D. Van der Spoel, E. Lindahl, B. Hess, G. Groenhof, A. Mark, and H. Berendsen, GROMACS: Fast, flexible, and free, J. Comput. Chem. 26, 1701 (2005).

[31] J. Barrat and L. Bocquet, Large slip effect at a nonwetting fluid-solid interface, Phys. Rev. Lett. 82, 4671 (1999).

[32] S. M. Dammer and D. Lohse, Gas Enrichment at Liquid-Wall Interfaces, Phys. Rev. Lett. 96, 206101 (2006).

[33] M. Nijmeijer, C. Bruin, A. Bakker, and J. Van Leeuwen, Wetting and drying of an inert wall by a fluid in a molecular-dynamics simulation, Phys. Rev. A 42, 6052 (1990).

[34] J. S. Rowlinson and B. Widom, Molecular Theory of Capillarity (Dover publications, London, 1982). 
[35] J.-P. Hansen and I. R. McDonald, Theory of Simple Liquids (Academic Press, Waltham, MA, 2006).

[36] G. J. Merchant and J. B. Keller, Contact Angles, Phys. Fluids A 4, 477 (1992).

[37] J. H. Snoeijer and B. Andreotti, A microscopic view on contact angle selection, Phys. Fluids 20, 057101 (2008).

[38] A. Marmur, Line tension and the intrinsic contact angle in solid-liquid-fluid systems, J. Colloid Interface Sci. 186, 462 (1997).

[39] B. M. Borkent, S. M. Dammer, H. Schönherr, G. J. Vancso, and D. Lohse, Superstability of Surface Nanobubbles, Phys. Rev. Lett. 98, 204502 (2007).

[40] M. A. Hampton and A. V. Nguyen, Nanobubbles and the nanobubble bridging capillary force, Adv. Colloid Interface Sci. 154, 30 (2010).

[41] M. P. Brenner and D. Lohse, Dynamic Equilibrium Mechanism for Surface Nanobubble Stabilization, Phys. Rev. Lett. 101, 214505 (2008).

\section{Appendix}

\section{Geometric interpretation of line tension}

Figure 7.13 shows by illustration how the free energy associated with the liquid-liquid interactions of a wedge-shaped liquid interface sitting on a solid can be decomposed in its bulk, surface and contact line contributions. $F_{L L}$ shows the total free energy of the liquid-liquid interactions, which is the interaction of the liquid in the wedge with itself. For clarity, we separated the two domains in the first row spatially, but in reality they of course overlap since they are the same volume of liquid. First, we decompose the integral domain in the bulk energy contribution (wedge shape $\otimes$ infinite volume): $F_{\text {bulk. }}$. The surplus that has to be subtracted is shown in the top right of Fig. 7.13, because one has to compensate for the areas where no liquid is present. From this surplus we extract the surface contributions. Note that the liquid wedge has two surfaces: the liquid-vapour interface and the liquid-solid interface, which are both represented by integration of the wedge (dotted area) with an infinite half-space, resulting in the total surface energy term. The third row shows what remains and is by definition 

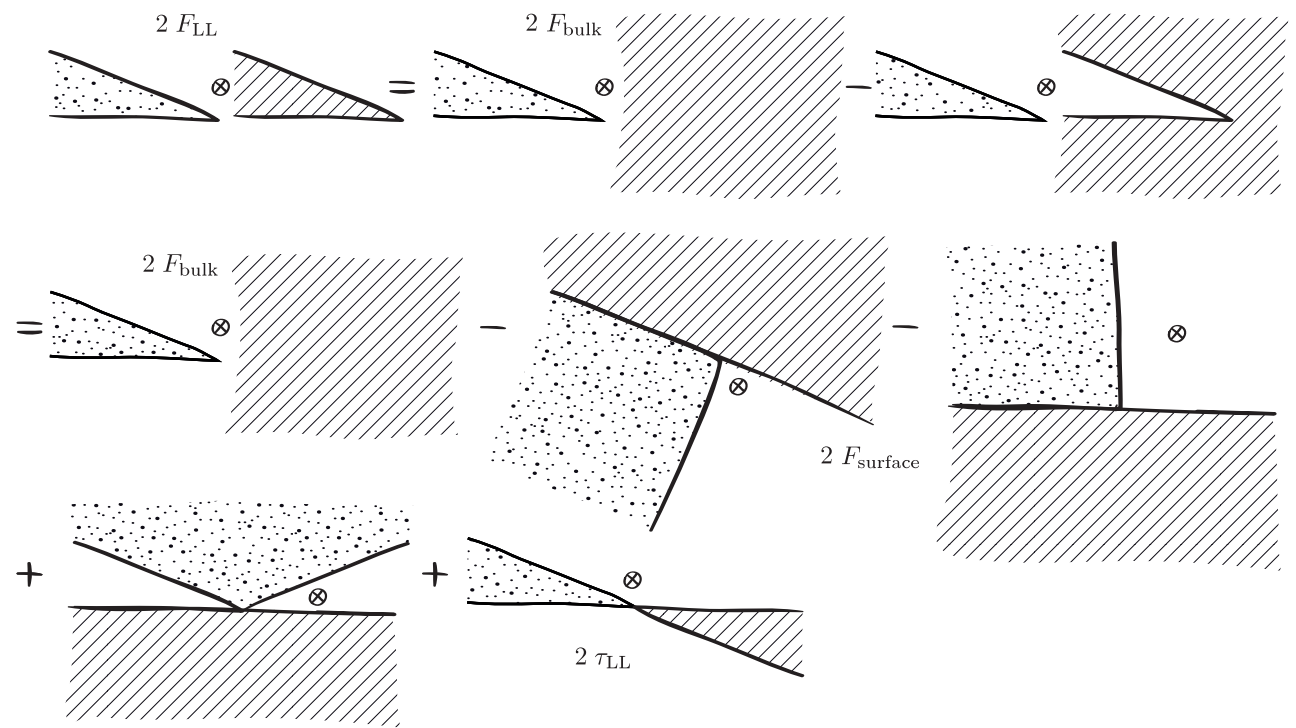

Figure 7.13: Integration domain for the liquid-liquid interaction energy decomposed in the bulk, surface, and line components. The dotted and striped regions represent the domains of integration for the variables $d \vec{r}_{1}$ and $d \vec{r}_{2}$, respectively, in the liquid-liquid term of (7.21). The remainder after subtracting the bulk energy $\left(F_{\text {bulk }}\right)$ and surface energy $\left(F_{\text {surface }}\right)$ is the free energy associated with liquid-liquid line tension $\left(\tau_{L L}\right)$. Note that the two line-tension contributions shown here can be combined into the integration domains shown in Fig. 7.12.

(Eq. (7.1)) the line tension. These integration domains can be simplified and merged into the one shown in Fig. 7.12 (left).

To compute $\tau_{L S}$ we follow a similar route. Fig. 7.14 (left) shows the integration domain for $F_{L S}$ for a liquid wedge (dotted) in contact with a solid (striped). The right two panels directly give the decomposition into the surfacic component (solid half-space $\otimes$ liquid half-space, over the solidliquid interface), and the remainder which is the line tension component $\left(\tau_{L S}\right)$. There is no bulk energy term since we are dealing with two separate (and spatially separated) phases. 


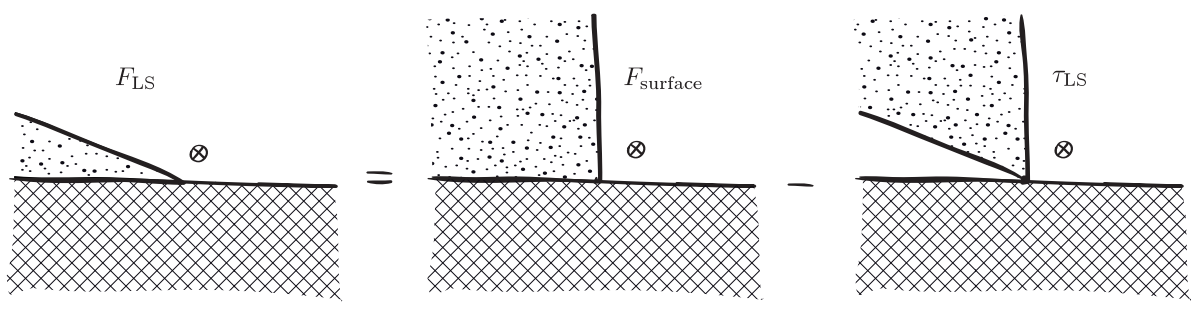

Figure 7.14: Integration domain for the liquid-solid interaction energy (left). This integration domain can be decomposed in the corresponding surface energy contribution $\left(F_{\text {surface }}\right)$ and the free energy associated with liquid-solid line tension $\left(\tau_{L S}\right)$. The dotted region represents the integration variable $d \vec{r}_{1}$ in the liquid-solid term of (7.21) and the double striped region the integration variable $d \vec{r}_{2}$ in the same equation. 


\section{Elastocapillarity at the nanoscale: on the coupling between elasticity and surface energy in soft solids *}

The capillary forces exerted by liquid drops and bubbles on a soft solid are directly measured using molecular dynamics simulations. The force on the solid by the liquid near the contact line is not oriented along the liquid vapor interface nor perpendicular to the solid surface, as usually assumed, but points towards the liquid. It is shown that the elastic deformations induced by this force can only be explained if, contrary to an incompressible liquid, the surface stress is different from the surface energy. Using thermodynamic variations we show that the the surface stress and the surface energy can both be determined accurately by measuring the deformation of a slender body plunged in a liquid. The results obtained from molecular dynamics fully confirm those recently obtained experimentally [Marchand et al. Phys. Rev. Lett. 108, 094301 (2012)] for an elastomeric wire.

*Published as: J.H. Weijs, B. Andreotti, and J.H. Snoeijer, "Elasto-capillarity at the nanoscale: on the coupling between elasticity and surface energy in soft solids", Soft Matter 9, 8494 (2013). 


\subsection{Introduction}

As largely demonstrated in the last two decades, elasticity plays an important role in surface physics. Phenomena such as surface reconstruction [1, 2], surface segregation [3], surface adsorption [4], elastic instabilities [5], self assembly [6, 7], and nanostructuration [8] of crystalline solids are directly induced by surface stresses. In parallel, and almost without any connection, the elastic deformations of sheets and rods [9-16] as well as gels and elastomers induced by capillary forces have been evidenced and investigated [17-33]. It has remained unclear to what extent it is important to distinguish surface tension from surface stress for these elastocapillary phenomena.

The definition and properties of surface stresses can be derived from thermodynamics, atomistic models and mechanics. These approaches are complementary and should in principle be consistent with one another. The simplest situation is an interface between a condensed phase and its vapor. Let us consider an extensive quantity, the density of which varies across the interface over the scale of a few molecular sizes. At a macroscopic scale, the density can be seen as homogeneous on both sides of the interface. However, the extensive quantity then presents an interfacial excess. For instance, the free energy presents an interfacial excess called the "surface energy", denoted $\gamma$. Dividing now the total volume of the condensed phase and its vapor into two subsystems with a dividing plane normal to the interface, the mechanical force between the two subsystems also presents an excess quantity called the "surface stress", which throughout the paper we refer to as $Y$. This is a force per unit length acting parallel to the interface, originating from molecular interactions.

In the very particular situation where the condensed phase is an incompressible liquid, it can be shown from the virtual work principle that the surface stress and the surface energy are strictly equal, i.e. $Y=\gamma$. The surface stress and surface energy are then unified into a single name "surface tension", and it is common to address capillary problems using either the thermodynamic, or the mechanical route [34,35]. In a solid, by contrast, the surface energy a priori depends on the strain in the bulk and yields an additional elastic contribution to the surface stress $[5,36]$. More precisely, the difference between the surface stress and the surface energy is the derivative of the surface energy with respect to the strain. This result is known as the Shuttleworth equation $[5,36]$, 


$$
\mathrm{Y}_{i j}=\gamma_{i j}+\frac{\partial \gamma_{i j}}{\partial \epsilon},
$$

where $\epsilon$ is the bulk strain parallel to the interface. The subscripts refer to the phases $i$ and $j$ on both sides of the interface. Indeed, the Shuttleworth equation also applies when the interface separates two condensed phases composed of different molecules. This is highly relevant for wetting phenomena, for which one naturally deals with liquid-solid interfaces. Such interfaces present an excess free energy $\gamma_{S L}$ that, according to (8.1), is different from the surface stress $Y_{S L}$. Once again, the surface stress is the excess force parallel to the interface and can be measured at the edge of any control volume that includes the interface. Importantly, the force $Y_{S L}$ is exerted on a subsystem composed two types of molecules, solid and liquid. From a molecular perspective, $\mathrm{Y}_{S L}$ is the resultant of all types of molecular forces: solid-liquid, solid-solid and liquid-liquid interactions.

The aim of this paper is to explore the difference between surface energy and surface stress in the case of soft solids that are partially wetted by a liquid. A paradigmatic example of this effect consists of a drop (or bubble) on a deformable solid [17, 18, 22-24, 28-33], as shown in Fig. 8.1. This problem has recently been explored experimentally and theoretically, with contradicting interpretations emerging from microscopic and macroscopic descriptions [24, 27-30, 32, 33, 37]. Indeed, we expect a particularly strong manifestation of the difference between surface energy and surface stress near a three-phase contact line. On the one hand, the liquid-vapor interface is characterized by a surface tension, i.e. $\gamma_{L V}=\mathrm{Y}_{L V}$, and Young's law for the equilibrium contact angle

$$
\gamma_{L V} \cos \theta=\gamma_{S V}-\gamma_{S L}
$$

only involves the surface energies. On the other hand, the mechanical equilibrium of the solid involves the surface stresses $Y_{S V} \neq \gamma_{S V}$ and $Y_{S L} \neq \gamma_{S L}$.

In this work, we reveal the connection between elastocapillary interactions at the nanoscale and the thermodynamic concepts of surface energy and surface stress. In Section 8.2, we perform Molecular Dynamics simulations of drops and bubbles on soft substrates, for which we can accurately determine the elastic deformations and the liquid-on-solid forces. We find that the deformation below a drop is markedly different from the deformation below a bubble (Fig. 8.1): we measure a tangential force oriented towards the liquid side, even for contact angles of $\theta=90^{\circ}$ for which $\gamma_{S V}=\gamma_{S L}$. 

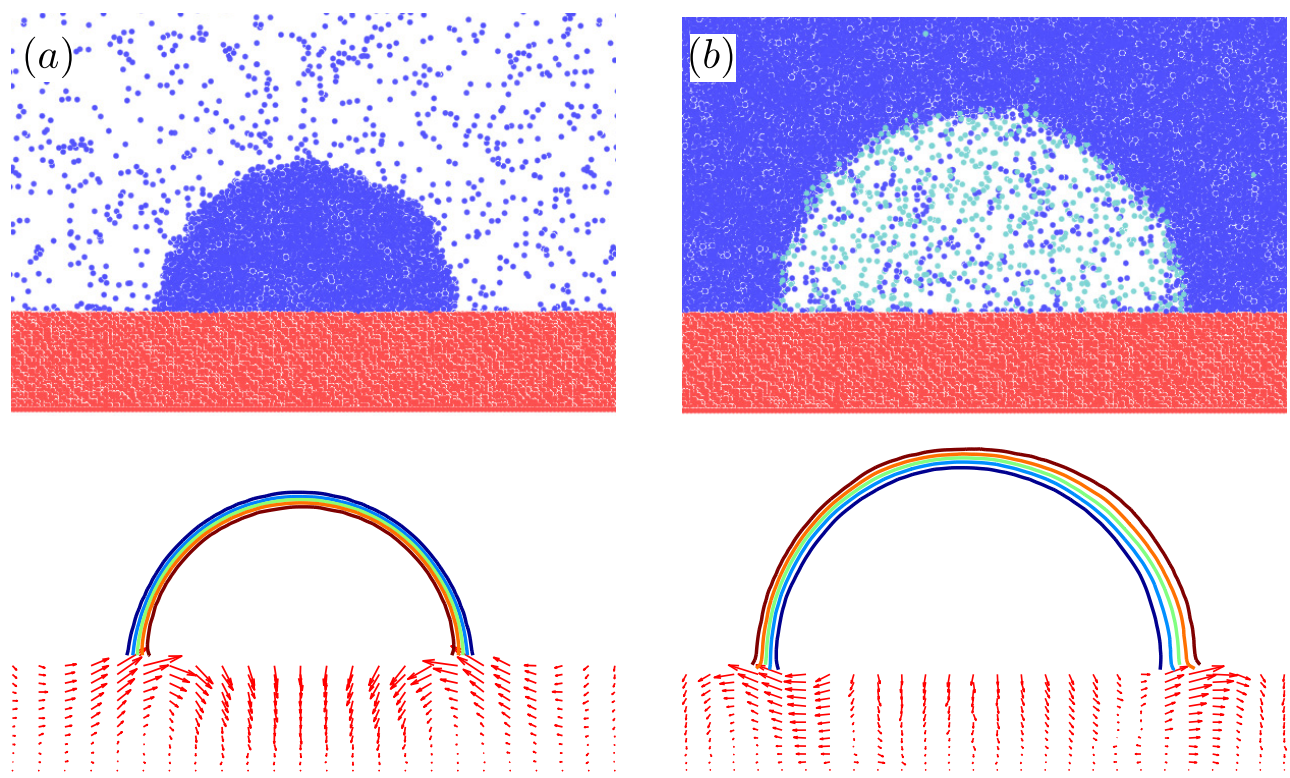

Figure 8.1: Molecular dynamics simulations of a drop (a) and a bubble (b) on a deformable substrate, both with contact angle $\theta=90^{\circ}$, hence $\gamma_{S L}=\gamma_{S V}$. (a) Drop on a soft substrate. Top: Snapshot, blue particles are liquid atoms, red particles are solid atoms. Bottom: Local displacement (red arrows) of the solid due to the presence of the liquid drop (shown by liquid isodensity contours). (b) Bubble on a soft substrate. Top: Snapshot, red and blue particles are the same as in (a), cyan particles are gas atoms. Bottom: Local displacement (red arrows) of the solid due to the presence of the bubble (shown by liquid isodensity contours). Note that the tangential displacement near the contact line is different between both situations: in the drop case the solid is pulled inwards whereas in the bubble case the solid is pulled outwards.

Can one explain this tangential force by invoking surface stresses? In Section 8.3 we therefore develop a purely thermodynamic view, in the case of a plate partially immersed in a liquid, and compare this directly to Molecular Dynamics simulations. Finally, we conclude in Section 8.4 by relating the tangential forces to the difference between surface energies and stresses. 


\subsection{Drops and bubbles}

\subsubsection{Molecular Dynamics}

The aim of this paper is to investigate the basic mechanisms controlling elastocapillary interactions in model situations. We have therefore performed Molecular Dynamics simulations using simple interactions for both the liquid and the soft solid, in a quasi-2D geometry. The simulations have been performed using the GromaCs software package [38]. The liquid consists of a Lennard-Jones fluid whose pair interaction potential is given by:

$$
\phi_{i j}(r)=4 e_{i j}\left[\left(\frac{d_{i j}}{r}\right)^{12}-\left(\frac{d_{i j}}{r}\right)^{6}\right] .
$$

Here, $e_{i j}$ and $d_{i j}$ are the interaction strength and range between particle types $i$ and $j$, respectively. The potential is cut off at $5 d_{L L}$, where $d_{L L}$ is the liquid atom size. The simulations are performed in the NVT-ensemble (constant number of particles $N$, constant volume $V$, and constant temperature $T$ using the thermostat described in [39]). The solid consists of atoms placed on a cubic lattice of 25 layers, with harmonic springs connecting each atom to its neighbour and next-nearest neighbour. The lattice spacing $a=0.8 d_{L L}$, while all spring constants are taken equal with $k=38.5 \cdot e_{L L} / d_{L L}^{2}$. There are no Lennard-Jones solid-solid interactions, hence the solid atoms only interact with each other through the harmonic springs. The solid (S) interacts with the liquid (L) through Lennard-Jones interactions. By varying the solidliquid interaction, we explore drops of different equilibrium contact angles as in chapter 7 . In the simulations for a gas bubble, we added gas atoms $(G)$ that also interact according to a Lennard-Jones potential. The addition of gas atoms in the bubble case is required to prevent the bubble from collapsing immediately, as would be the case for a vapour bubble. The Lennard-Jones interaction parameters are given in Table 8.1. In the following paragraph we define the relevant dimensionless numbers.

From a macroscopic view, the elastocapillary deformations arise from a balance between surface tension $\gamma$ and the elastic modulus $E$ (for simplicity of notation we use $\gamma$ for the liquid-vapor surface tension $\gamma_{L V}$ ). The ratio of these parameters $\gamma / E$ gives the elastocapillary length, which sets the scale of the elastic deformations. Our quasi-two-dimensional simulations have plane-strain conditions, in which case the relevant elastic modulus reads $E=\tilde{E} /\left(1-v^{2}\right)$, where $\tilde{E}$ is the Young's modulus and $v$ the Poisson ratio. 
In terms of lattice parameters in our simulations, we find $E=15 k /(8 a)$. To quantify the relative softness of the substrate, one can compare the elastocapillary length to the (liquid) atomic size $d_{L L}$, which gives the dimensionless quantity $\gamma /\left(E d_{L L}\right)$. Whenever this quantity is small, the deformations are weak and one should recover the contact angles according to Young's law [37]. Here we measure the liquid-vapour surface tension $\gamma$ in a separate system using a Kirkwood-Buff integral over the stress-anisotropy near the interfaces $\gamma=\int\left(p_{N}-p_{T}(z)\right) d z$ where, $p_{N}$ is the (constant) thermodynamic pressure in the system, and $p_{T}$ the tangential (relative to the interface) component of the stress-tensor which deviates from $p_{N}$ near the liquid-vapour interface [40-42]. We find, using the method outlined in [41] to determine the local pressure that $\gamma=0.78 e_{L L} / d_{L L}^{2}$. In this work, therefore, the parameter $\gamma /\left(E d_{L L}\right)=8.6 \cdot 10^{-3}$ is indeed small, meaning that all elastic displacements are much smaller than atomic size (small elastic strains), and allows the use of linear elasticity theory. The typical length scale of thermal fluctuations in the solid, $\sqrt{k_{B} T / k}$, compared to the elastocapillary deformation described before provides another dimensionless quantity $\frac{\gamma \sqrt{k}}{E \sqrt{k_{B} T}}=0.18$. The smallness of this parameter shows that the thermal fluctuations in the system are much larger than the deformation due to capillary forces. Still, as we will show, the displacement field can be measured very accurately in the simulations after averaging over time. Finally, we note that in SI-units, the chosen material properties correspond to real materials at $T=300 \mathrm{~K}$, $E=11 \mathrm{GPa}$, and $\gamma=3.1 \cdot 10^{-2} \mathrm{~J} / \mathrm{m}^{2}$. Surface tension coefficients of simple liquids typically lie between $0.02 \mathrm{~J} / \mathrm{m}^{2}$ (ethanol) and $0.07 \mathrm{~J} / \mathrm{m}^{2}$ (water). Young's modulus of crystalline solids is typically around $E=100 \mathrm{GPa}$ while it can be much lower for elastomers (between $10^{-2}$ and $10^{-1} \mathrm{GPa}$ for rubber) and gels (down to $1 \mathrm{kPa}$ ) whose elasticity is entropic.

The small strains in the solid (smaller than thermal fluctuations) are measured by calculating the time-averaged displacements (relative to the center of mass of the droplet or bubble) of the solid atoms compared to a base state, obtained from a simulation of the same solid in vacuum. As we are interested in the influence of the liquid on the solid, this procedure allows us to exclude effects associated with the presence of a solid-vacuum interface. The contact angle of the droplet and of the bubble are measured by determining the time-averaged density field of the liquid and the position of the Gibbs interface. We refer the reader to our previous work [42] for technical details. The circular fit to this liquid-vapor/gas interface is extrapolated to the solid, which provides the contact angle. 


\begin{tabular}{lll}
\hline Interaction pair $i, j$ & $\frac{e_{i j}}{k_{B} T}(T=300 \mathrm{~K})$ & $d / \mathrm{nm}$ \\
\hline LL & 1.2 & 0.34 \\
SL & varied & 0.34 \\
SS & 0 & 0 \\
GG & 0.4 & 0.5 \\
SG & 0.004 & 0.34 \\
LG & 0.7 & 0.42 \\
\hline
\end{tabular}

Table 8.1: Lennard-Jones Interaction parameters for the MD-simulations for Liquid, Solid and Gas atoms. With these values, the liquid-vapour surface tension is $\gamma=$ $3.1 \cdot 10^{-2} \mathrm{~J} / \mathrm{m}^{2}$.

\subsubsection{Elastocapillary deformations}

To illustrate that surface energy is not sufficient to characterize elastocapillary deformations, we first consider a case where $\gamma_{S V}=\gamma_{S L}$, such that the contact angle of the liquid is close to $90^{\circ}$. The contact angle can be adjusted by tuning only the Lennard-Jones interaction $e_{S L}$, while keeping the liquid parameters $\left(e_{L L}, \sigma_{L L}, \sigma_{S L}\right)$ fixed. This way, the liquid properties are unchanged except for the interaction with the solid. Note that, in general, the surface stresses will be different from the surface energies, and thus $\mathrm{Y}_{S V} \neq \mathrm{Y}_{S L}$.

Figure 8.1 shows the elastic deformation in the solid below a liquid drop (panel a), and below a bubble filled with gas (panel b). Since $\theta=90^{\circ}$, the shape of the liquid-vapor interface, characterized by the iso-density profiles, is very similar in both cases. By contrast, the elastic deformations are markedly different, as can be seen from the vector field (red arrows): while below the drop one observes a tangential displacement towards the center of the drop, the displacements below the bubble are oriented outwards. This surprising outcome has important consequences. The drop and the bubble are perfectly symmetric from the point of view of the surface energies, since $\gamma_{S V}=\gamma_{S L}$ in this case. Yet, this symmetry is not reflected in the surface displacements: the deformations are not invariant under an inversion of the phases. Instead, the solid is always pulled towards the liquid side of the contact line (not only for the case $\theta=90^{\circ}$ ). Therefore, an elastocapillary description based on constant surface energies (i.e. on surface tensions) is not sufficient to describe the elastic deformations below a drop or below a bubble. 


\subsubsection{Capillary traction and contact line force}
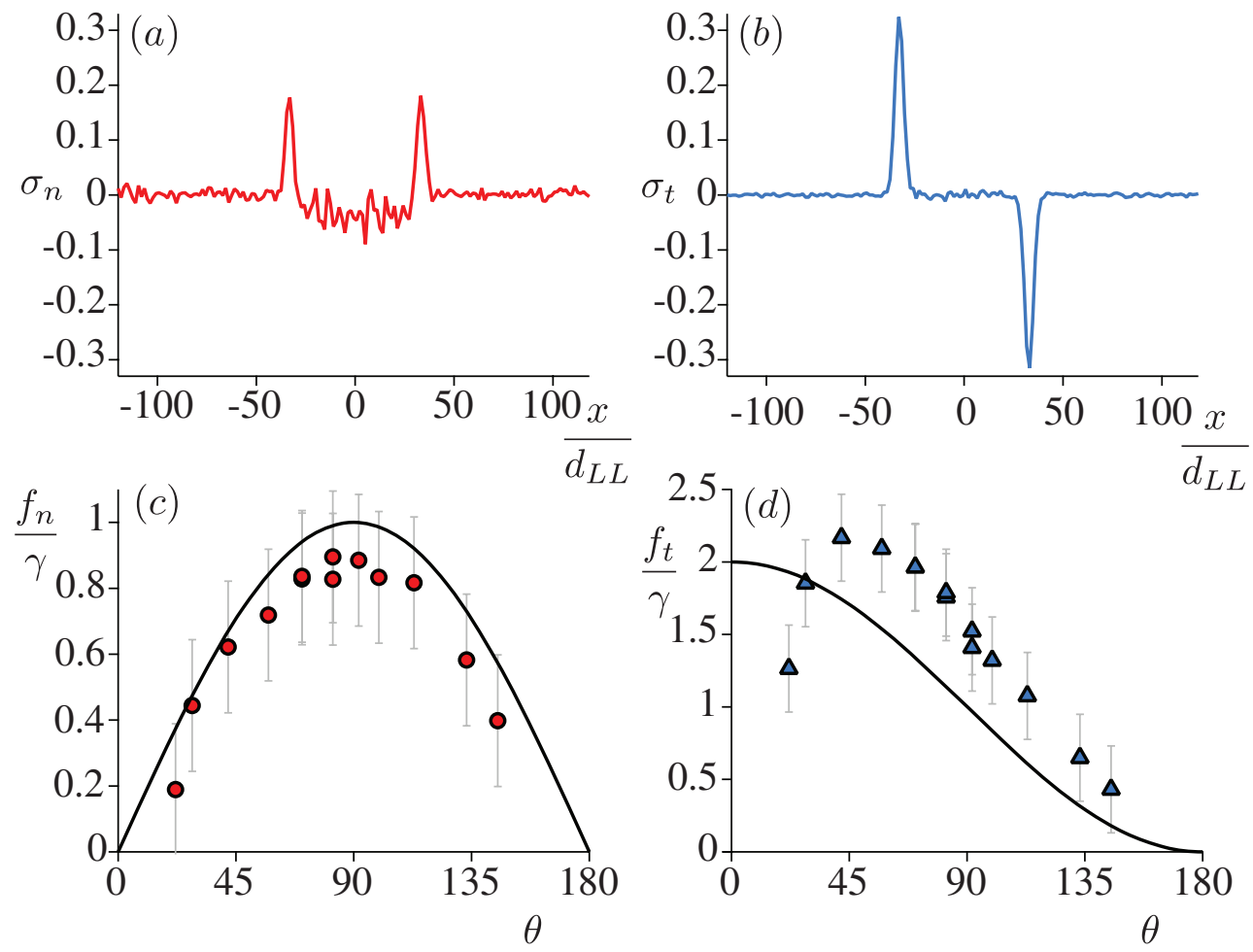

Figure 8.2: Capillary traction: the liquid on solid forces per unit area of the substrate measured in molecular dynamics of a droplet on a substrate $\left(\theta=82^{\circ}\right)$. (a) Normal component of the force per area by the liquid on the solid, $\sigma_{n}$. The two peaks correspond to the contact lines, where the solid gets pulled up. The region between the peaks corresponds to the liquid-solid interface, where the solid gets pushed down due to the Laplace pressure. (b) Tangential component of the force per area by the liquid on the solid, $\sigma_{t}$. There is only a force near the contact lines, and the force is directed towards the interior of the droplet. (c) Normal component of the total force by the liquid on the solid due to the contact line at varying $\theta$. The solid line corresponds to $\sin \theta$. (d) Tangential component of the total force by the liquid on the solid due to the contact line at varying $\theta$. The solid line corresponds to $1+\cos \theta$. The low values for small contact angles $\theta<40^{\circ}$ are likely due to finite size effects in the simulations.

Before turning to a fully thermodynamic description of elastocapillary deformations in Sec. 8.3, we first quantify the capillary liquid on solid forces at the nanoscale. Note that for a complete description of the bulk deforma- 
tions one requires both liquid on solid forces and any solid on solid forces that are present in the surface layer. However, since the asymmetry is caused by the liquid on solid forces we first quantify the liquid on solid forces only. The common feature of the deformation below the drop and the bubble is that, below the contact line, the tangential deformations are oriented towards the side of the liquid phase (Fig. 8.1). This is consistent with the predictions of the Density Functional Theory in the sharp interface approximation $[29,35]$, which is based on a microscopic description of the interactions. The mechanism for this asymmetry is that the long-ranged attraction by the liquid molecules creates a resultant force on the solid that is biased towards the liquid: the solid is more strongly attracted by the phase of highest density. This resultant force ultimately determines the elastic deformations, and is responsible for breaking the symmetry between drops and bubbles in Fig. 8.1.

In Molecular Dynamics, we can of course directly quantify this effect by measuring the time-averaged forces that all liquid molecules exert on the solid molecules. Since we wish to reveal the capillary traction (force per area) that the liquid induces on the solid, we integrate over the vertical depth of the solid. In practice, the direct liquid-solid interaction only acts in the superficial layers of the solid, due to the short range of Lennard-Jones (van der Waals) interactions $\left(\sim r^{-6}\right)$. The resulting capillary traction below a drop is shown in Fig. 8.2. First, the curve in Fig. 8.2a shows the normal traction, $\sigma_{n}$, of the liquid on the solid in the case $\theta=82^{\circ}$. As expected, we observe a large traction that is localized near the two contact lines: this corresponds to the "pulling" action of the contact line. The width of the peak is a few molecular sizes and reflects the width of the liquid-vapor interface. At the center of the drop one observes a slightly negative traction, corresponding to the Laplace pressure in the bulk of the drop. As the drop is in equilibrium, this Laplace pressure perfectly balances the upward stress at the contact line: the total liquid-on-solid normal force is zero. Using macroscopic thermodynamics we can estimate the normal force exerted by the liquid on the solid in the vicinity of the contact line. For drop sizes that are much larger than the width of the peak in $\sigma_{n}$ (which coincides with the thickness of the liquidvapour interface), we can separate the capillarity forces into a contribution per unit area, $P=\gamma / R$, and a perfectly localized force per unit contact line $f_{n}$. Using that the width of the drop $2 R \sin \theta$ and that the total force vanishes, one predicts that the strength of the force on the solid near the contact line should be $f_{n}=\gamma \sin \theta$ (per unit contact line). We can test this macroscopic 
prediction in our simulations. First, we determine the liquid-vapor surface tension $\gamma$ from an independent calibration, as described in Sec. 8.2.1. Then, we integrate the normal stress over the peak located around the contact line, yielding the total normal force per unit contact line $f_{n}$. The result for different contact angles $\theta$ is shown in Fig. 8.2c. The results of MD simulations are consistent with a contact line force in the normal direction $f_{n}=\gamma \sin \theta$, shown in solid line.

Similarly, we can determine the tangential capillary traction on the solid, denoted $\sigma_{t}$. The result is shown in Fig. 8.2b. We find a positive traction at the contact line located on the left (i.e. pointing towards the right), and a negative traction at the contact line located on the right (i.e. pointing towards the left). Indeed, we identify an effective liquid-on-solid force that is oriented towards the interior of the drop, i.e. into the liquid phase. This tangential force is the reason why the elastic displacements point towards the interior of the drop. Again, we quantify the total force exerted on the solid near the contact line from the integral of the peaks, $f_{t}$. The resulting tangential force per unit contact line is shown in Fig. 8.2d, as a function of the contact angle. We observe a nonzero inward tangential force for all angles. This can be understood from the left-right symmetry breaking below the contact line: the solid atoms are attracted much more strongly by the highdensity liquid phase than by the low-density vapor phase. As the strength of the solid-liquid interaction is directly quantified by the work of adhesion, $\gamma+\gamma_{S V}-\gamma_{S L}$, one expects below a liquid/vapour/solid triple line $[35]^{+}$:

$$
f_{t}=\gamma+\gamma_{S V}-\gamma_{S L}=\gamma(1+\cos \theta) \text {. }
$$

This equation is shown as the solid line in Fig. 8.2d: it indeed captures the features of the tangential force, which is always positive, i.e. oriented towards the liquid side, and nicely describes the magnitude and trend with the contact angle. This also explains the difference between the deformations below a drop and a bubble.

In conclusion, our simulations clearly demonstrate the existence of a tangential capillary force exerted on the region of the solid below the contact line. This force has a strong influence on the elastic deformation below a drop or bubble. This effect is usually ignored in the literature on elastocapillarity $[17,18,22,28,30,32]$, likely due to the fact that in the case of an

\footnotetext{
${ }^{\dagger}$ In case the vapor is replaced by a second, immiscible liquid, there will be second "work of adhesion" contribution. This yields another tangential force on the solid that is biased toward the second liquid, and weakens the asymmetry.
} 
incompressible liquid (or solid) this tangential force is exactly balanced by solid on solid forces and therefore is not transmitted to the bulk. However, in general for $v \neq 1 / 2$, this is not the case and the tangential force (which is always pointed towards the liquid phase, see Fig. 8.2d) needs to be taken into account when considering the elastic deformation.

\subsection{Partially immersed solid}

From the preceding section is it clear that the elastic deformation below a contact line results from the detailed interactions (capillary and elastic) at the nanoscale. We will now address the problem in a macroscopic framework, where we relate the elastic displacements to purely thermodynamic concepts. In particular, the goal is to express the microscopic interactions discussed in section 8.2 directly in terms of the excess quantities $\gamma_{i j}$ and $Y_{i j}$. For this, we consider an long elastic plate that is partially immersed in a liquid - see Fig. 8.3. This geometry was studied before experimentally using a thin elastomeric wire [43]. The experiment revealed that the elastic strain in the "wet" part of the solid was very different from the strain in the "dry" part. Here we analyze this geometry using the thermodynamic concepts of surface stress and then compare it directly to Molecular Dynamics.

The central result of this section is that the vertical strain above the contact line, $\tilde{\varepsilon}_{+}$, couples to the surface energies, while the strain below the contact line, $\tilde{\varepsilon}_{-}$, is determined by the surface stresses:

$$
\begin{aligned}
\frac{W E}{2} \tilde{\varepsilon}_{+} & =\gamma_{S V}-\gamma_{S L} \\
\frac{W E}{2} \tilde{\varepsilon}_{-} & =\mathrm{Y}_{S V}-\mathrm{Y}_{S L} .
\end{aligned}
$$

Here $W$ is the width of a two-dimensional elastic plate, $E$ is the elastic modulus, while the reference state for the strain is the completely dry solid. By determining the strain inside the plate, one thus directly measures the difference between surface energies and surface stresses. This method will be applied in our Molecular Dynamics simulations.

\subsubsection{Thermodynamics: strain above and below meniscus}

To derive (8.5) and (8.6), we consider the free energy of an elastic plate that is partially submerged in a liquid bath with the contact line at $z=0$ (Fig. 8.3). 


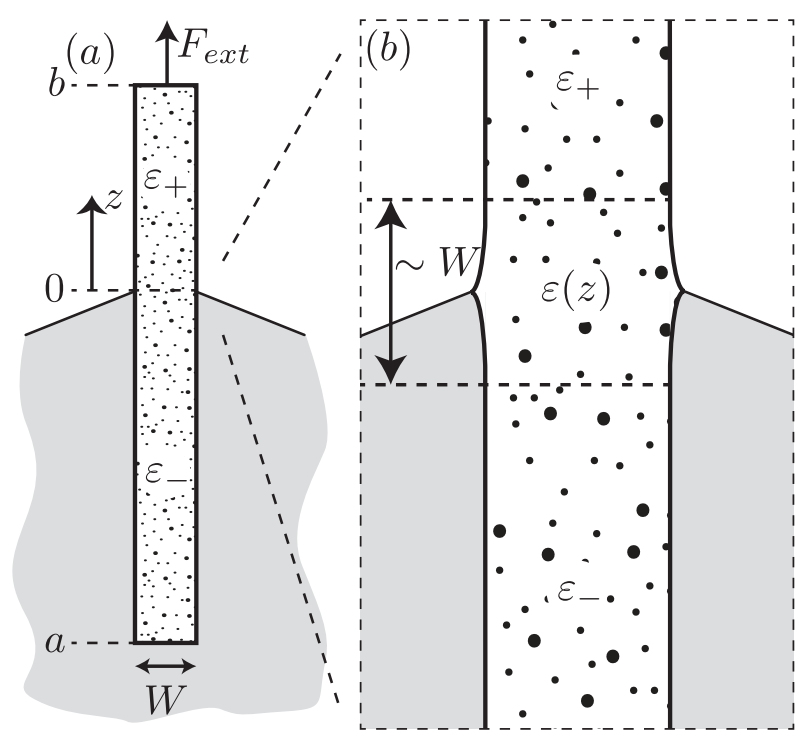

Figure 8.3: Partially immersed plate of width $W$, held at equilibrium by an external force $F_{\text {ext }}$. (a) The plate is partially wetted, with the contact line located at $z=0$. There is a homogeneous strain above and below the contact line: $\varepsilon_{+}$and $\varepsilon_{-}$, respectively. (b) Zoom around the contact line. Near the contact line exists a transition region of the strain from $\varepsilon_{+}$to $\varepsilon_{-}$. The transition occurs over a length $\sim W$.

We will assume that the elastocapillary length $\gamma / E$ is much smaller than the plate width $W$, which in turn is much smaller than the total plate length $L$ :

$$
\frac{\gamma}{E} \ll W \ll L .
$$

The first assumption allows one to consider the solid-liquid and solid-vapor interfaces to be flat with respect to other scales in the problem, as can be seen from the zoom near the contact line in Fig. 8.3b. The second assumption implies that, apart from the region directly below the contact line of width $W$, the strain is homogeneous and characterized by a constant value for $\epsilon=d u_{z} / d z$, where $u_{z}$ is the vertical displacement field. We denote the strains above and below the contact line as $\epsilon_{+}$and $\epsilon_{-}$respectively; the contribution to the energy of the region directly below the contact line is sub-dominant by a factor $W / L$. Outside the contact line region the strains can be considered small, since $\epsilon_{ \pm} \sim \gamma / E W \ll 1$. Under these assumptions, we arrive at the following free energy functional of the system (per unit length): 


$$
\begin{aligned}
\mathcal{F}= & 2 \int_{a}^{z_{c l}} d z\left[\gamma\left(1+h^{\prime 2}\right)^{1 / 2}+\gamma_{S L}\right]+2 \int_{z_{c l}}^{b} d z \gamma_{S V} \\
& +W \int_{a_{0}}^{z_{c l}} d z \frac{1}{2} E \epsilon_{-}^{2}+W \int_{z_{c l}}^{b_{0}} d z \frac{1}{2} E \epsilon_{+}^{2} \cdot
\end{aligned}
$$

Here $a$ and $b$ denoted the bottom and top positions of the plate, but note that the elastic energy should be taken over a domain of fixed length $L_{0}=b_{0}-a_{0}$, with reference positions $a_{0}$ and $b_{0}$ [44]. We allow for variations of the shape of the liquid-vapor interface $h(z)$, the position of the contact line $z_{c l}$, the top and bottom positions of the plate $b$ and $a$, and the elastic strains $\epsilon_{ \pm}$. The equilibrium conditions follow from

$$
\delta \mathcal{F}=F_{\text {ext }} \delta b
$$

which equates the change in energy to the work done by the external force. Note that the positions $b$ and $a$ are linked by the constraint

$$
b-a=L_{0}+\int_{a_{0}}^{b_{0}} d z \epsilon .
$$

First, we derive the equilibrium conditions for the liquid, by considering variations of the liquid-vapor interface $\delta h(z)$, with $\delta b=\delta \epsilon_{+}=\delta \epsilon_{-}=0$. From geometry near the contact line, this implies a variation of the contact line position according to $\delta h\left(z_{c l}\right)=-h^{\prime}\left(z_{c l}\right) \delta z_{c l}$. One thus obtains

$$
\begin{aligned}
& \frac{1}{2} \delta \mathcal{F}=0= \\
& \delta z_{c l}\left[\gamma\left(1+h^{\prime 2}\right)^{1 / 2}+\gamma_{S L}-\gamma_{S V}+\frac{1}{2} E W\left(\epsilon_{-}^{2}-\epsilon_{+}^{2}\right)\right]_{z_{c l}} \\
& +\delta h\left[\frac{\gamma h^{\prime}}{\left(1+h^{\prime 2}\right)^{1 / 2}}\right]_{z_{c l}}-\int d z \frac{\gamma h^{\prime \prime}}{\left(1+h^{\prime 2}\right)^{3 / 2}} \delta h .
\end{aligned}
$$

The integral expresses the Laplace pressure condition for the liquid-vapor interface. The terms $\sim \epsilon_{ \pm}^{2}$ arise from the fact that a variation of $z_{c l}$ does not affect the contact line zone, but just gives an exchange of the elastic energies of the dry and wet parts respectively (similar to the exchange of surface 
energies $\left.\gamma_{S L}-\gamma_{S V}\right)$. Collecting the terms from the boundary condition, using $\delta h\left(z_{c l}\right)=-h^{\prime}\left(z_{c l}\right) \delta z_{c l}$, one finds the condition for the equilibrium contact angle

$$
\begin{aligned}
\cos \theta & =\frac{\gamma_{S V}-\gamma_{S L}}{\gamma}+\frac{1}{2} \frac{E W}{\gamma}\left(\epsilon_{+}^{2}-\epsilon_{-}^{2}\right) \\
& =\frac{\gamma_{S V}-\gamma_{S L}}{\gamma}+\mathcal{O}\left(\frac{\gamma}{E W}\right) .
\end{aligned}
$$

where we replaced $\cos \theta=1 /\left(1+h^{\prime 2}\right)^{1 / 2}$. This shows that for $\gamma / E W \ll 1$ one recovers Young's law for the liquid contact angle with respect to the undeformed solid.

Next, we explore the elastic degrees of freedom of the plate. For convenience, we now choose the contact line position as the reference altitude: $z_{c l}=0$. Using Eq. (8.8), one writes the free energy $\mathcal{F}_{p}$ of the plate and its interfaces with the liquid and the vapor:

$$
\mathcal{F}_{p}=2 b \gamma_{S V}\left(\epsilon_{+}\right)-2 a \gamma_{S L}\left(\epsilon_{-}\right)+\frac{1}{2} W E\left(b_{0} \epsilon_{+}^{2}-a_{0} \epsilon_{-}^{2}\right) .
$$

Here we made explicit the strain dependence of surface energies, which is necessary for solid interfaces. Due to the relations

$$
b=b_{0}\left(1+\epsilon_{+}\right), \quad a=a_{0}\left(1+\epsilon_{-}\right), \quad a_{0}=b_{0}-L_{0},
$$

there are only three independent variables. We choose here to parametrize the problem using $b_{0}, \epsilon_{+}$and $\epsilon_{-}$. From this we can write the total variation:

$$
\begin{aligned}
\frac{1}{2} \delta \mathcal{F}_{p}= & \delta b_{0}\left[\gamma_{S V}-\gamma_{S L}+\frac{1}{2} E W\left(\epsilon_{+}^{2}-\epsilon_{-}^{2}\right)\right] \\
& +b_{0} \delta \epsilon_{+}\left[\gamma_{S V}+\frac{\partial \gamma_{S V}}{\partial \epsilon}+\frac{E W}{2} \epsilon_{+}\right] \\
& -a_{0} \delta \epsilon_{-}\left[\gamma_{S L}+\frac{\partial \gamma_{S L}}{\partial \epsilon}+\frac{E W}{2} \epsilon_{-}\right]
\end{aligned}
$$

where, after the variation, we replaced $b=b_{0}$ and $a=a_{0}$ owing to the smallness of the strains. For the same reason, we also anticipate that the terms of order $E W \epsilon_{ \pm}^{2}$ can be neglected in the following steps.

The variation of the plate energy must be balanced with the work done by the external force 


$$
\delta \mathcal{F}_{p}=F_{\text {ext }} \delta b=F_{\text {ext }} \delta b_{0}+F_{\text {ext }} b_{0} \delta \epsilon_{+} .
$$

Combining (8.15) and (8.16), gives the three equilibrium conditions. The variation of $b_{0}$ gives the familiar expression for the external force needed to hold the plate, i.e.

$$
F_{\text {ext }}=2\left(\gamma_{S V}-\gamma_{S L}\right)
$$

Using this expression, we find that the strains must follow

$$
\begin{aligned}
& E W \varepsilon_{+}=-2 \frac{\partial \gamma_{S V}}{\partial \varepsilon}-2 \gamma_{S L} \\
& E W \varepsilon_{-}=-2 \frac{\partial \gamma_{S L}}{\partial \varepsilon}-2 \gamma_{S L} .
\end{aligned}
$$

The reference state for the strain, $\varepsilon_{0}$, will be the plate in contact with the vapour only. This reference can be derived in a similar way by minimizing the free energy of a plate in vapour (i.e. replacing $\gamma_{S L}$ by $\gamma_{S V}$ in either of the above equations):

$$
E W \varepsilon_{0}=-2 \frac{\partial \gamma_{S V}}{\partial \varepsilon}-2 \gamma_{S V}
$$

The final step is to subtract the reference state from (8.18) and (8.19), and substitute the Shuttleworth equation $Y_{i j}=\frac{\partial \gamma_{i j}}{\partial \varepsilon}+\gamma_{i j}$ :

$$
\begin{aligned}
& \tilde{\varepsilon}_{+} \equiv \varepsilon_{+}-\varepsilon_{0}=\frac{2}{E W}\left(\gamma_{s v}-\gamma_{s l}\right) \\
& \tilde{\varepsilon}_{-} \equiv \varepsilon_{-}-\varepsilon_{0}=\frac{2}{E W}\left(Y_{s v}-Y_{s l}\right) .
\end{aligned}
$$

Indeed, this is the result anticipated in (8.5) and (8.6). In conclusion, the immersion of a slender body allows one to determine accurately both surface energies and surface stresses [43].

\subsubsection{Molecular Dynamics}

We now test this method in a MD simulation of an immersed plate. Figure 8.4 shows a snapshot of the simulation, where the solid plate is partially immersed into a liquid reservoir. The material of the plate is the same as that used in the previous drop and bubble simulation, except that it is stiffer: $k_{p}=10 k$, such that $E_{p}=110 \mathrm{GPa}$ whereas the liquid surface tension is unchanged: $\gamma=3.1 \cdot 10^{-2} \mathrm{~J} / \mathrm{m}^{2}$. The container that holds the liquid consists 


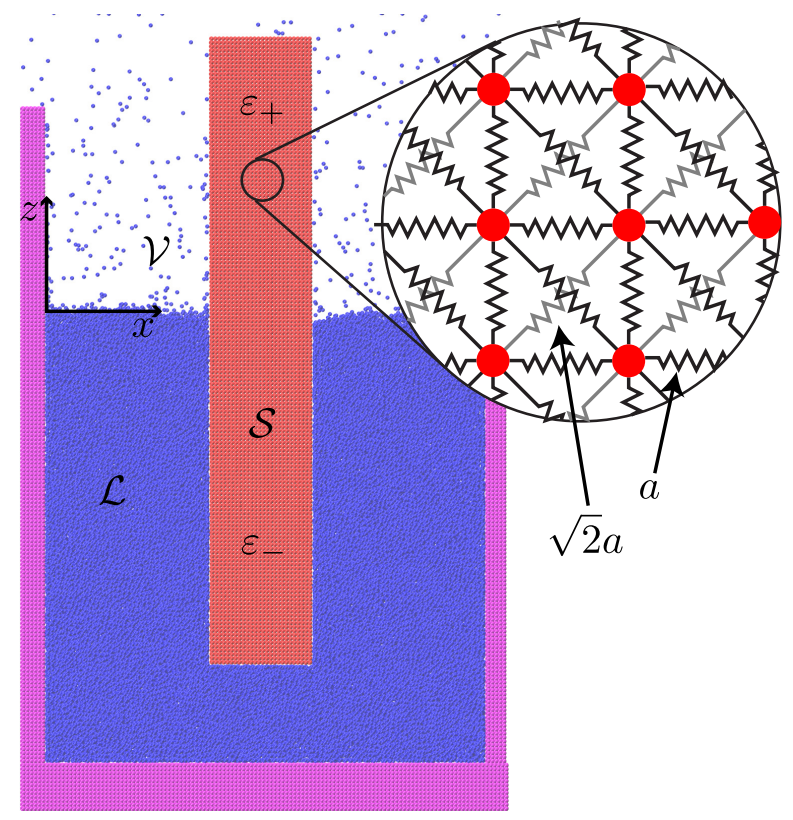

Figure 8.4: Snapshot from an MD-simulation of a plate ( $W / a=26$ atoms wide) partially submerged in a liquid bath. The liquid-solid interaction energy $e_{S L}$ was chosen such that $\theta=90^{\circ}$. Comparing the time-averaged $z$-position of the solid atoms to the time-averaged position of the reference system (plate in vacuum), allows for the local displacement $u_{z}(z)$ to be measured.

of the same material as the plate, except that the container is not allowed to deform by fixing the atoms to their initial positions. To avoid a curved liquid meniscus, and hence a difference in pressure on the wetted and dry regions of the plate, we consider again $\theta=90^{\circ}$. In this case $\gamma_{S V}=\gamma_{S L}$ and according to (8.5) we expect $\tilde{\varepsilon}_{+}=0$. Before each simulation we equilibrated the solid in a vacuum to have a well-defined reference state.

Figure 8.5 shows an example of the vertical elastic displacement, $u_{z}(z)$, that is induced after immersion of the plate. The slope of this curve directly gives the strain $\tilde{\varepsilon}=d u_{z} / d z$. Indeed, we observe very different strains above and below the contact line, which allows for a determination of $\tilde{\varepsilon}_{+}$and $\tilde{\varepsilon}_{-}$. In this example the top part of the plate is hardly deformed, as expected from (8.5) for this situation where $\gamma_{S V}=\gamma_{S L}$. By contrast, the lower part of the plate displays a negative strain, $\tilde{\varepsilon}_{-}<0$, corresponding to a compression of the bottom part of the wire. Using (8.6), this reveals a difference in surface stresses, $\mathrm{Y}_{S V} \neq \mathrm{Y}_{S L}$, despite the equality of surface energies. 


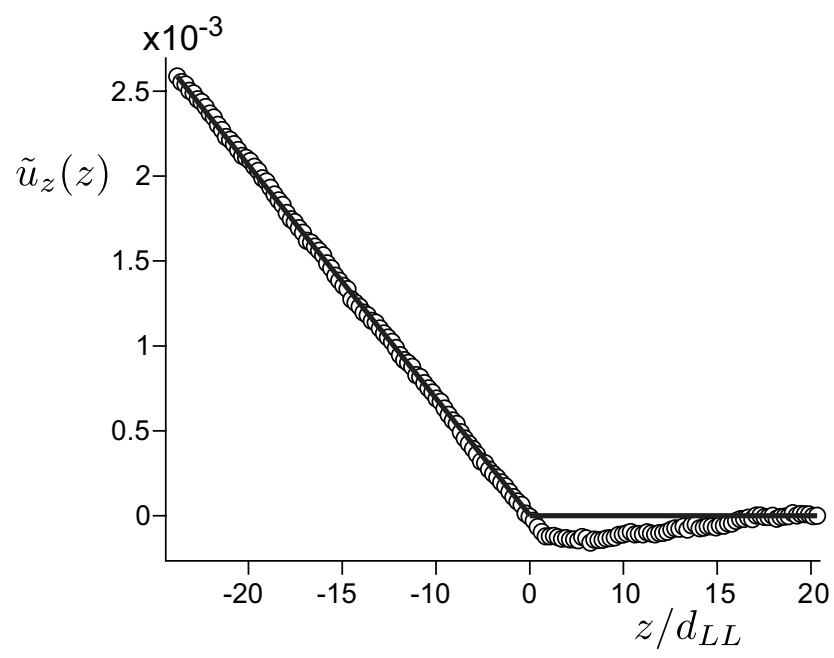

Figure 8.5: MD measurement of the relative displacement $\tilde{u}_{z}(z)$ for plate width $W=8.8 d_{L L}$. The derivative of this slope gives the strain $\tilde{\varepsilon}(z)$. The contact line is located at $z=0$, where a clear jump in $\tilde{\varepsilon}(z)$ is observed (from $\tilde{\varepsilon}_{-}$to $\tilde{\varepsilon}_{+}$, see also Figs. 8.3 and 8.4.) This signifies an imbalance in surface stresses $\left(\mathrm{Y}_{S L} \neq \mathrm{Y}_{S V}\right)$ even though $\gamma_{S L}=\gamma_{S V}$ for $\theta=90^{\circ}$.

To quantify the difference $Y_{S V}-Y_{S L}$, we repeated the simulations with plates of various widths $W$. The resulting $\tilde{\varepsilon}_{+}$and $\tilde{\varepsilon}_{-}$are plotted as a function of $1 / W$ (Fig.8.6). Above the contact line we indeed find a vanishing strain $\tilde{\varepsilon}_{+}$for all plate thicknesses, within the error bars of the simulation. Below the contact line we observe a nearly linear dependence on $1 / W$, as predicted by (8.6). This confirms that our system is large enough to apply thermodynamics and continuum elasticity. The slope of the curve is measured at $\frac{d \tilde{\varepsilon}_{-}}{d(1 / W)}=-(1.4 \pm 0.3) \cdot 10^{-3} d_{L L}$. This slope can be related to the surface stress via Eq. (8.6) and we find, for this specific solid and liquid pair:

$$
\mathrm{Y}_{S V}-\mathrm{Y}_{S L}-\left(\gamma_{S V}-\gamma_{S L}\right)=\mathrm{Y}_{S V}-\mathrm{Y}_{S L}=-(0.81 \pm 0.17) \gamma
$$

This surface stress difference is clearly not a negligible effect: it is of the same order as the liquid-vapor surface tension.

\subsection{Conclusions}

We have shown from thermodynamic considerations that the elastic deformation of a partially wetted solid crucially depends on the difference be- 


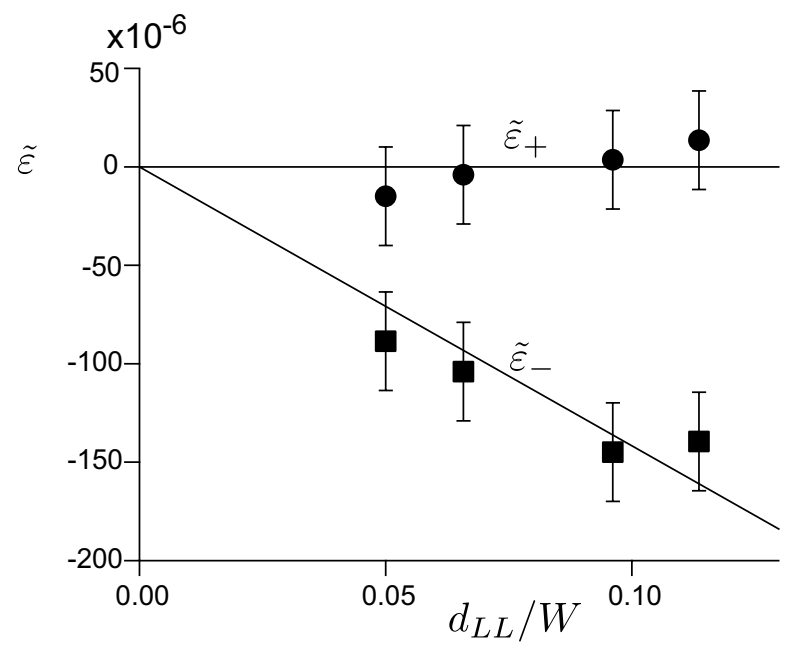

Figure 8.6: MD measurements of the vertical strains $\tilde{\varepsilon}_{+}$(circles) and $\tilde{\varepsilon}_{-}$(squares) as functions of the inverse plate width $d_{L L} / W$. Within error, there exists no strain above the contact line $\left(\varepsilon_{+}\right)$. This is expected for $\theta=90^{\circ}$. Below the contact line, however, the solid is compressed due to an imbalance of the surfaces stresses at the contact line $\left(\mathrm{Y}_{S L} \neq \mathrm{Y}_{S V}\right)$. The slope of this curve quantifies the magnitude of this imbalance, Eq. (8.6).

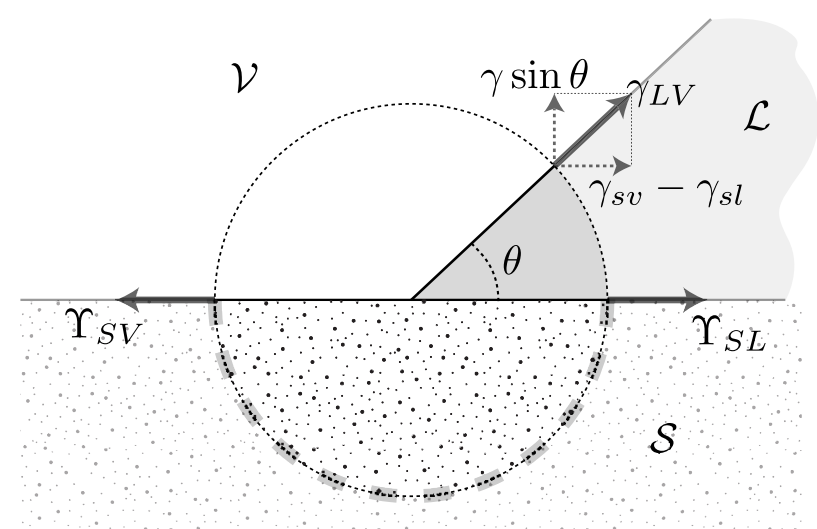

Figure 8.7: Stresses acting on the circular control volume around the contact line. Note that for solids generally $Y_{S X} \neq \gamma_{S X}$, hence there exists an imbalance of the interfacial stresses in both the normal and tangential directions. This imbalance is counteracted by elastic stresses in the solid, along the thick gray dashed line. 
tween surface stress and surface energy. This result is confirmed using Molecular Dynamics simulations, revealing how deformations emerge from interactions at the nanoscale. To complete the picture of elastocapillary interactions, we finally give a purely mechanical interpretation of our findings.

Describing the partially immersed wire of Fig. 8.3 using continuum elasticity, a discontinuity of strain implies a discontinuity of stress across the contact line, in the direction parallel to the solid interface. This means that the contact line region must exert a tangential force $f_{t}^{e l}$ on the bulk elastic material [43]. The magnitude of the tangential force experienced by bulk elasticity is proportional to $\epsilon_{+}-\epsilon_{-}$, and therefore reads

$$
f_{t}^{e l}=\left(\mathrm{Y}_{S L}-\mathrm{Y}_{S V}\right)-\left(\gamma_{S L}-\gamma_{S V}\right) \text {. }
$$

This residual force accounts for all interactions that are transmitted across the surface layers to the bulk elastic, including the solid-solid interactions. It is therefore important to distinguish $f_{t}^{e l}$ from $f_{t}$ measured in Fig. 8.2: while the latter only included the liquid-on-solid forces, the surface stress captures the total excess surface force and includes all superficial interactions.

Figure 8.7 shows how the residual force $f_{t}^{e l}$ arises due to the imbalance of surface stresses in the vicinity of the contact line. When discussing the forces near the contact line, it is absolutely critical to explicitly specify the material system to which the forces are applied: a different choice of control volume will lead to different forces [35, 43]. Here we consider a macroscopic control volume that includes the three-phase contact line, as indicated by the dotted circle. As this includes the three interfaces, one can directly represents the surface stresses $Y_{S V}, Y_{S L}$ and $Y=\gamma$ as indicated by the solid arrows. Interestingly, the equilibrium contact angle does not involve the surface stresses of the solid, but rather the surface energies $\gamma_{S L}$ and $\gamma_{S V}$. This is not inconsistent with Fig. 8.7, because Young's law represents an equilibrium (a minimal free energy from the thermodynamic perspective and a balance of forces from the mechanical point of view) inside the liquid only, and thus requires a different control volume that does not include the solid [35]. As a consequence, the surface stresses in Fig. 8.7 do not balance in the direction parallel to the solid, but yields a nonzero tangential force $f_{t}^{e l}=\gamma \cos \theta+\mathrm{Y}_{S L}-\mathrm{Y}_{S V}$, in agreement with (8.22). Similarly, the surface stresses yield a resultant normal force

$$
f_{n}^{e l}=\gamma \sin \theta
$$

To restore mechanical equilibrium inside the control volume, both $f_{n}^{e l}$ and 


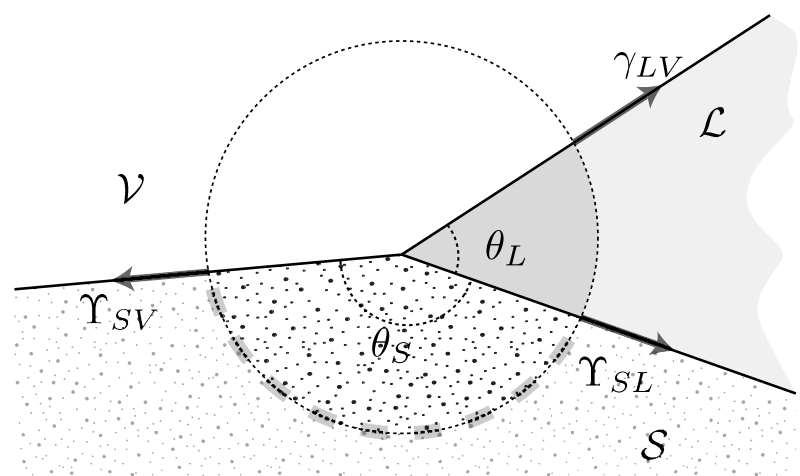

Figure 8.8: Stresses acting on the circular control volume around the contact line, in case of strong solid deformation $(\gamma / E a \gg 1)$. The liquid equilibrates at an angle $\theta_{L}$ that is a function of the solid angle $\theta_{S}$ and the surface energies $\gamma_{S L}, \gamma_{S V}$. Since for solids generally $Y_{S X} \neq \gamma_{S X}$, the liquid equilibration does not coincide with a balance of the interfacial stresses. This imbalance is counteracted by elastic stresses in the solid, along the thick gray dashed line.

$f_{t}^{e l}$ must be balanced by elastic stresses that are exerted along the along the grey dashed circular section in Fig. 8.7. The tangential component vanishes only when the surface energies and surface stresses are equal.

A similar observation can be made for very soft solids, for which the solid deforms into a "cusp" shape with a solid angle $\theta_{S}<\pi[28,30,37]$. The cusp develops when $\gamma / E d_{L L} \gg 1$ [37], and thus corresponds to cases much softer than in our Molecular Dynamics. Figure 8.8 shows the surface stresses near the contact line on such a strongly deformed solid. For given value of $\theta_{S}$, the equilibration of the liquid angle $\theta_{L}$ involves only the surface energies $\gamma_{S L}, \gamma_{S V}$ and not the stresses $Y_{S L}, Y_{S V}$. In general, the liquid equilibrium will therefore not coincide with the balance of surface stresses on the circular control volume in Fig. 8.8 [37]: Residual elastic stress will arise whenever surface energies differ from surface stresses.

The key parameter for wetting of soft materials is thus the difference between surface energies and surface stresses, as in equation (8.22). Experimentally, the strain discontinuity across the contact line for the partially immersed wire gives direct access to this difference. The recently suggested method to determine the surface stress from contact angles assumes a perfect balance of surface stresses [30], and therefore incorrectly assumes that elastic stress can be ignored for the balance that determines the contact angle. This method, therefore, applies only when there is no difference between sur- 
face energies and stresses. Theoretically, we can now put upper and lower bounds on the tangential force. Clearly, the simulations in Fig. 8.1 show that the tangential force originates from the breaking of left/right symmetry near the contact line, biased towards the side of the high-density liquid. The maximum possible residual force $f_{t}^{e l}$ should therefore be the liquid-on-solid force $\gamma+\gamma_{S V}-\gamma_{S L}$. This maximum arises whenever the solid-solid interactions in the surface layer do not counteract this effect and the full tangential force is transmitted to the bulk substrate. This was referred to as the "vectorial force transmission model" in previous work [37]. Another extreme limit corresponds to the "normal force transmission model", for which the surface layers completely screen out any tangential stress. This is the case, for example, when the substrate is another liquid (i.e. an oil drop floating on liquid). A liquid can of course not sustain any shear, which means that the symmetry-breaking of interactions is counteracted by self-interactions inside the liquid substrate. This once more agrees with (8.22), since for liquid-liquid interfaces $Y_{i j}=\gamma_{i j}$. Hence, we conclude

$$
0 \leq f_{t}^{e l} \leq \gamma+\gamma_{S V}-\gamma_{S L}
$$

or equivalently

$$
\gamma_{S L}-\gamma_{S V} \leq \mathrm{Y}_{S L}-\mathrm{Y}_{S V} \leq \gamma
$$

Future work should be dedicated to determining how the difference between $Y$ and $\gamma$ exactly depend on the material dimensionless parameters: the solid Poission ratio the ratio of the elastocapillary length to the atomic size and the ratio of the elastocapillary length to the thermal length. The results from our Molecular Dynamics are very close to the upper bound: the surface stress difference was found slightly smaller than the liquid-vapor surface tension $\gamma$. A similar conclusion can be drawn from the experimental results reported in [43], where an elastomeric wire that was partially immersed in a liquid. The symbol $\Gamma$ used in this previous work can now be identified with $Y_{S L}-Y_{S V}$, which was found identical to $\gamma$ within experimental uncertainty [43]. To further explore the difference between surface energy and surface stress, it would be interesting to directly measure the tangential displacements inside soft substrates, e.g. using confocal microscopy [30], and compare the deformation below a drop and a bubble. 


\section{References}

[1] C. Bach, M. Giesen, H. Ibach, and T. Einstein, Stress relief in reconstruction, Phys. Rev. Lett. 78, 4225 (1997).

[2] A. Filippetti and V. Fiorentini, Reconstructions of $\operatorname{Ir}(110)$ and (100): An ab initio study, Surf. Sci. 377, 112 (1997).

[3] P. Wynblatt and R. Ku, Surface-energy and solute strain-energy effects in surface segregation, Surf. Sci. 65, 511 (1977).

[4] H. Ibach, The relation between the strain-dependence of the heat of adsorption and the coverage dependence of the adsorbate induced surface stress, Surf. Sci. 556,71 (2004).

[5] P. Muller and A. Saul, Elastic effects on surface physics, Surf. Sci. Rep. 54, 157 (2004).

[6] O. Alerhand, D. Vanderbilt, R. Maede, and J. Joannopoulos, Spontaneous formation of stress domains on crystal-surfaces, Phys. Rev. Lett. 61, 1973 (1988).

[7] J. Metois, A. Saul, and P. Muller, Measuring the surface stress polar dependence, Nat. Mater. 4, 238 (2005).

[8] E. Ramé, Encyclopedia of Surface and Colloid Science (Marcel Dekker, New York, 2002), pp. 3602-3618.

[9] J. Bico, B. Roman, L. Moulin, and A. Boudaoud, Elastocapillary coalescence in wet hair, Nature 432, 690 (2004).

[10] C. Py, P. Reverdy, L. Doppler, J. Bico, B. Roman, and C. N. Baroud, Capillary Origami: Spontaneous wrapping of a droplet with an elastic sheet, Phys. Rev. Lett. 98, 156103 (2007).

[11] A. Boudaoud, J. Bico, and B. Roman, Elastocapillary coalescence: aggregation and fragmentation with a maximal size, Phys. Rev. E 76, 060102 (2007).

[12] C. Py, P. Reverdy, L. Doppler, J. Bico, B. Roman, and C. N. Baroud, Capillarity induced folding of elastic sheets, Eur. Phys. J. Spec. Top. 166, 67 (2009). 
[13] J. W. van Honschoten, J. W. Berenschot, T. Ondarcuhu, R. G. P. Sanders, J. Sundaram, M. Elwenspoek, and N. R. Tas, Elastocapillary fabrication of three-dimensional microstructures, Appl. Phys. Lett. 97, 014103 (2010).

[14] B. Roman and J. Bico, Elasto-capillarity: deforming an elastic structure with a liquid droplet, J. Phys. Cond. Matt. 22, 493101 (2010).

[15] F. Chiodi, B. Roman, and J. Bico, Piercing an interface with a brush: collaborative stiffening, Europhys. Lett. 9o, 44006 (2010).

[16] J. Hure, B. Roman, and J. Bico, Wrapping an adhesive sphere with an elastic sheet, J. Phys. Chem. B 106, 174301 (2011).

[17] G. Lester, Contact angles of liquids at deformable solid surfaces, J. Colloid Sci. 16, 315 (1961).

[18] A. Rusanov, Theory of wetting of elastically deformed bodies. 1. Deformation with a finite contact-angle, Colloid J. USSR 37, 614 (1975).

[19] S. Yuk and M. Jhon, Contact angles on deformable solids, J. Colloid Interface Sci. 110, 252 (1986).

[20] M. Shanahan, The influence of solid micro-deformation on contact-angle equilibrium, J. Phys. D-Appl. Phys. 2o, 945 (1987).

[21] A. Carre, J. Gastel, and M. Shanahan, Viscoelastic effects in the spreading of liquids, Nature 379, 432 (1996).

[22] L. White, The contact angle on an elastic substrate. 1. The role of disjoining pressure in the surface mechanics, J. Colloid Interface Sci. 258, 82 (2003).

[23] R. Pericet-Camara, E. Bonaccurso, and K. Graf, Microstructuring of polystyrene surfaces with nonsolvent sessile droplets, ChemPhysChem 9, 1738 (2008).

[24] R. Pericet-Camara, A. Best, H.-J. Butt, and E. Bonaccurso, Effect of capillary pressure and surface tension on the deformation of elastic surfaces by sessile liquid microdrops: An experimental investigation, Langmuir 24, 10565 (2008).

[25] S. Mora, T. Phou, J.-M. Fromental, L. M. Pismen, and Y. Pomeau, Capillarity Driven Instability of a Soft Solid, Phys. Rev. Lett. 105, 214301 (2010). 
[26] M. Sokuler, G. K. Auernhammer, M. Roth, C. Liu, E. Bonaccurso, and H.-J. Butt, The Softer the Better: Fast Condensation on Soft Surfaces, Langmuir 26, 1544 (2010).

[27] F. Leonforte and M. Mueller, Statics of polymer droplets on deformable surfaces, J. Chem. Phys. 135, 214703 (2011).

[28] E. R. Jerison, Y. Xu, L. A. Wilen, and E. R. Dufresne, Deformation of an Elastic Substrate by a Three-Phase Contact Line, Phys. Rev. Lett. 106, 186103 (2011).

[29] S. Das, A. Marchand, B. Andreotti, and J. H. Snoeijer, Elastic deformation due to tangential capillary forces, Phys. Fluids 23, 072006 (2011).

[30] R. W. Style, R. Boltyanskiy, Y. Che, J. S. Wettlaufer, L. A. Wilen, and E. R. Dufresne, Universal Deformation of Soft Substrates Near a Contact Line and the Direct Measurement of Solid Surface Stresses, Phys. Rev. Lett. 110, 066103 (2013).

[31] Y. Wang, B. Bhushan, and X. Zhao, Nanoindents produced by nanobubbles on ultrathin polystyrene films in water, Nanotechnology 20, 045301 (2009).

[32] R. W. Style and E. R. Dufresne, Static wetting on deformable substrates, from liquids to soft solids, Soft Matter 8, 7177 (2012).

[33] L. Limat, Straight contact lines on a soft, incompressible solid, Eur. Phys. J. E 35, 134 (2012).

[34] P.-G. de Gennes, F. Brochard-Wyart, and D. Quere, Capillarity and wetting phenomena: drops, bubbles, pearls, waves (Springer, New York, 2004).

[35] A. Marchand, J. H. Weijs, J. H. Snoeijer, and B. Andreotti, Why is surface tension a force parallel to the interface?, Am. J. Phys. 79, 999 (2011).

[36] R. Shuttleworth, The surface tension of solids, Proc. Phys. Soc., London Sect. A 63, 444 (1950).

[37] A. Marchand, S. Das, J. H. Snoeijer, and B. Andreotti, Contact Angles on a Soft Solid: From Young's Law to Neumann's Law, Phys. Rev. Lett. 109, 236101 (2012).

[38] D. Van der Spoel, E. Lindahl, B. Hess, G. Groenhof, A. Mark, and H. Berendsen, GROMACS: Fast, flexible, and free, J. Comput. Chem. 26, 1701 (2005). 
[39] G. Bussi, D. Donadio, and M. Parrinello, Canonical sampling through velocity rescaling, J. Chem. Phys. 126, 014101 (2007).

[40] J. Kirkwood and F. Buff, The Statistical Mechanical Theory of Surface Tension, J. Chem. Phys. 17, 338 (1949).

[41] M. Nijmeijer, C. Bruin, A. Bakker, and J. Van Leeuwen, Wetting and drying of an inert wall by a fluid in a molecular-dynamics simulation, Phys. Rev. A 42, 6052 (1990).

[42] J. H. Weijs, A. Marchand, B. Andreotti, D. Lohse, and J. H. Snoeijer, Origin of line tension for a Lennard-Jones nanodroplet, Phys. Fluids 23, 022001 (2011).

[43] A. Marchand, S. Das, J. H. Snoeijer, and B. Andreotti, Capillary Pressure and Contact Line Force on a Soft Solid, Phys. Rev. Lett. 108, 094301 (2012).

[44] L. D. Landau and E. M. Lifshitz, Theory of Elasticity 3 rd edn. (Butterworth-Heinemann, Oxford, 1986). 


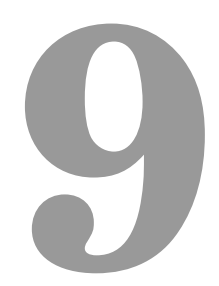

\section{Capillarity of soft amorphous solids: a microscopic model for surface stress *}

The elastic deformation of a soft solid induced by capillary forces crucially relies on the excess stress inside the solid-liquid interface. While for a liquid-liquid interface this "surface stress" is strictly identical to the "surface free energy", the thermodynamic Shuttleworth equation implies that this is no longer the case when one of the phases is elastic. Here we develop a microscopic model that incorporates enthalpic interactions and entropic elasticity, based on which we explicitly compute the surface stress and surface free energy. It is found that the compressibility of the interfacial region, through the Poisson ratio rather than by the Young's modulus, determines the difference between surface stress and surface energy. We highlight the consequence of this finding by discussing recent experiments on partially wetted soft substrates.

\subsection{Introduction}

In the last decades, surface effects in solid state physics have been widely investigated from the applied and fundamental point of view. An appropriate thermodynamic framework has been developed to describe the interplay

*Manuscript in preparation 
between elasticity and surface effects in crystalline solids [1-8]. An essential result is that the concept of surface tension cannot be applied without any caution to a solid condensed phase. This description is usually, however, not considered in the soft matter community, which has focused on problems of adhesion, elastocapillarity of slender bodies, deformation of an elastomer by a liquid drop, etc. [9-33]

As already pointed out in chapter 8 a correct treatment of elastocapillarity requires to distinguish between two excess interfacial quantities: the surface energy and the surface stress. Using a thermodynamical approach to derive the relation between surface energy and surface stress yields the well-known Shuttleworth-relation [34]:

$$
\mathrm{Y}_{A B}=\frac{\mathrm{d} \gamma}{\mathrm{d} \varepsilon}+\gamma
$$

with $\varepsilon$ the elastic strain parallel to the interface. From this relation it is immediately clear that for incompressible liquids, and only for such systems, the surface energy and surface stress are equal and are usually called "surface tension". The aim of this chapter is to adapt this framework previously proposed for crystalline solids, to the case of elastomers and gels: We will extend the concept of surface stress to the case of two condensed phases in contact. Specifically, we will propose a microscopic model for these macroscopic excess quantities, in the case of soft elastic amorphous solids, based on the density functional theory in the sharp interface approximation. We will show that the compressibility of the superficial layer, quantified by the Poisson ratio $v$, is the key characteristic of elastomers for elasto-capillary effects. The central result will be that

$$
\mathrm{Y}_{S L}=\frac{v}{1-v} \gamma_{S L}+\frac{1-2 v}{1-v}\left(\gamma_{S V}+\gamma_{L V}\right) .
$$

If the surface layer is perfectly incompressible, i.e. $v=1 / 2$, this gives a strict equality between surface energy and surface stress: $Y_{S L}=\gamma_{S L}$, as for liquid interfaces. The other extreme limit is that the stress directions perfectly decouple, i.e. $v=0$, for which $Y_{S L}=\gamma_{S V}+\gamma_{L V}$.

In Sect. 9.2 we derive a microscopic model to describe fluid and solid behaviour, which we use to derive $Y_{S L}$ in Sect. 9.3. The consequence of this result [Eq. (9.2)] will be discussed in detail in the discussion of Sect. 9.4, where we compare our findings to recent experiments and numerical results. 


\subsection{Microscopic model}

\subsubsection{Microscopic and macroscopic stress in a condensed phase}

We wish to define a model to compute the mechanical stresses that arise in the vicinity of a fluid-fluid or fluid-solid interface. These surface or capillary forces originate from molecular interactions, and predicting their strength thus calls for an approach that incorporates such microscopic interactions. Before developing this in detail, let us first consider the purely macroscopic viewpoint and define a macroscopic (or thermodynamic) stress tensor $\Sigma$. In absence of external fields, mechanical equilibrium condition is

$$
\vec{\nabla} \cdot \bar{\Sigma}=0 .
$$

For a planar interface with a normal in the $z$-direction, this thus implies that the normal stress $\Sigma_{z z}$ is constant and identical in both phases (assuming homogeneity in the $x, y$-directions). When the interface is curved, the macroscopic description requires a discontinuity of normal stress as a boundary condition at the interface, to account for the microscopic interactions in the surface layers. For a fluid-fluid interface, this can be expressed as the difference in thermodynamic pressure, $\Delta P=\gamma \kappa$, where $\gamma$ is the surface tension and $\kappa$ twice the mean curvature.

This macroscopic result can be contrasted with a purely microscopic approach that explicitly includes the molecular interactions. Microscopically, the interfacial region will be continuous, but one should still recover as a result that the bulk stress far away from a curved interface is different in the two phases. For fluid-fluid interfaces, such a microscopic framework has been developed based on Density Functional Theory using the so-called sharp-kink approximation. As will be further outlined in the next subsection, the key assumption of this theory is that the molecular interactions can be split in a long-range attractive part and a short-ranged repulsive interaction. The attraction is described by a (mean-field) potential $\phi$, while the repulsion is a contact stress described a microscopic stress tensor $\sigma$. The mechanical equilibrium equation then reads

$$
\vec{\nabla} \cdot \bar{\sigma}-\vec{\nabla} \phi=0 .
$$

The sharp-kink approximation assumes that the interface between the two phases is perfectly sharp, but that the interaction potential is long-ranged and extends across the interface - most notably, the potential varies only 
near the interface and $\vec{\nabla} \phi$ directly accounts for interfacial stresses. Since capillary effects are already included in this description, the boundary condition is now that normal stress $\sigma_{z z}$ is continuous across the interface. This model is very close to the original work by Laplace [35], who postulated an internal pressure to balance the strong attractive forces between molecules. For incompressible liquids near a straight interface, we will derive the following connection between macroscopic and microscopic stress:

$$
\Sigma_{i j}=\sigma_{i j}-(\phi+c) \delta_{i j} .
$$

One should bear in mind that even under atmospheric conditions, the repulsive stress $\sigma_{i j}$ is orders of magnitude larger than $\Sigma_{i j}$, as it balances the strong attractive interaction $\phi$. An estimate for the repulsive pressure is obtained by $\gamma / a$, where $a$ is the scale of the repulsion (a few Angstroms) and thus $p_{r} \sim 10^{8} \mathrm{~N} / \mathrm{m}^{2}$, as already estimated by Laplace [35].

The description above is valid for fluids. Below, we will generalize this formulation to account for elastic solids of which the elastic properties arise from entropic effects. In other words, the description focuses on solids such as gels and elastomers, which are polymer solutions in which there is no connected structure based on enthalpic (chemical) elastic effects. The goal is to derive an expression for the surface stress [Eq. (9.2)] from the microscopic interactions at various interfaces.

\subsubsection{Density functional theory in the sharp interface approxima- tion for liquids}

We consider a simple model of liquid based on a pair potential $\varphi$. The key idea of the Density Functional Theory is to express the grand potential $\Omega=U-T S-\mu N=F-\mu N$ as a functional of the particle density $\rho$ and to perform a functional minimisation for given values of the chemical potential $\mu$ and of the temperature $T$. The free energy $F$ is not known explicitly and needs to be built by integration, starting from the free energy of a known state. For a perturbation theory, one may choose hard spheres or the same system with only a repulsive potential, and then treat the van der Waals attraction as a corrective effect.

In the sharp interface approximation[36-38], one uses a standard vander-Waals expansion of the free energy around the hard-sphere reference system (whose pair potential is denoted $\varphi_{H S}(r)$ ), computed in the local den- 
sity approximation.

$$
\begin{aligned}
F[\rho] & =\int f_{H S}(\rho(\vec{r})) d \vec{r} \\
& +\frac{1}{2} \int_{0}^{1} d \lambda \int d \vec{r}_{1} \int d \vec{r}_{2} \rho\left(\vec{r}_{1}\right) \rho\left(\vec{r}_{2}\right) g_{\lambda}\left(\vec{r}_{1}, \vec{r}_{2}\right) \varphi\left(\left|\vec{r}_{2}-\vec{r}_{1}\right|\right)
\end{aligned}
$$

where $g_{\lambda}$ is the pair correlation function in a system of same geometry and same volume, for which the interaction is $\varphi_{\lambda}(r)=\varphi_{H S}(r)+\lambda\left[\varphi(r)-\varphi_{H S}(r)\right]$ above the sphere radius. One can use, for instance, a low density approximation for $g_{\lambda}$ :

$$
g_{\lambda}(r)=\exp \left(-\varphi_{\lambda}(r) / k T\right)
$$

We now apply the Gibbs interface idealization and assume that the density is homogeneous on both sides of the interface. We furthermore extend the description to several phases and introduce $\phi_{\alpha \beta}(\vec{r})$ the effective potential associated to the influence of phase $\alpha$ on phase $\beta$, at $\vec{r}$ :

$$
\phi_{\alpha \beta}(\vec{r})=\rho_{\alpha} \rho_{\beta} \int_{\mathcal{V}_{\alpha}} d \vec{r}^{\prime} \varphi_{\alpha \beta}\left(\left|\vec{r}-\vec{r}^{\prime}\right|\right) g_{r, \alpha \beta}\left(\left|\vec{r}-\vec{r}^{\prime}\right|\right) .
$$

Note that in general $\phi_{\alpha \beta} \neq \phi_{\beta \alpha}$, unless the domains of $\alpha$ and $\beta$ have an identical shape. Altogether, we get contribution to the free energy associated to the phase $\alpha$ as:

$$
F_{\alpha}=\int_{\mathcal{V}_{\alpha}}\left[p_{r}+\frac{1}{2} \phi_{L L}(\vec{r})+\phi_{S L}(\vec{r})\right] d \vec{r}
$$

The long-range attractive interaction between liquid and solid molecules gives rise to a potential $\phi_{S L}$ i.e. an energy per unit volume. The shortrange repulsive interactions are assumed to lead to an isotropic stress i.e. to a contact pressure $p_{r}$ [39].

Minimising the free energy, we obtain the (microscopic) equilibrium condition inside the liquid,

$$
\nabla\left(p_{r}+\phi_{L L}+\phi_{S L}\right)=0
$$

so that the total potential $p_{r}+\phi_{L L}+\phi_{S L}$ must be homogeneous [36, 37]. This is the same result as recovered in Eq. (9.5), as for liquids $\bar{\sigma}=-p_{r}$, and the macroscopic stress $\bar{\Sigma}$ is also homogeneous in the liquid phase. For liquids, therefore, $\bar{\sigma}=-p_{r}$. 
In this work, we work with planar interfaces only and therefore, for future reference, we define the potential $\phi$ in phase $\alpha$ due to the presence of a semi-infinite phase $\beta$ at distance $h$ from an interface as:

$$
\Pi_{\alpha \beta}(z)=\int_{-\infty}^{\infty} \int_{-\infty}^{\infty} \int_{h}^{\infty} \phi_{\alpha \beta}(|\mathbf{r}|) \mathrm{d} z \mathrm{~d} y \mathrm{~d} x,
$$

Note that, altough in general $\phi_{\alpha \beta} \neq \phi_{\beta \alpha}$ the equivalence $\Pi_{\alpha \beta}=\Pi_{\beta \alpha}$ does hold, since in that case the phases $\alpha$ and $\beta$ are similarly shaped (both are infinite halfspaces). Within this formalism we can readily compute the adhesion energies and hence the surface free energies. As the surface tensions are defined for flat interfaces between two semi-infinite phases, these energies can be expressed in terms of integrals over $\Pi$ and one finds [29]:

$$
\begin{aligned}
& \mathcal{A}_{A B}=-\int_{0}^{\infty} \Pi_{A B}(z) d z=\gamma_{A}+\gamma_{B}-\gamma_{A B} \\
& \mathcal{A}_{A A}=-\int_{0}^{\infty} \Pi_{A A}(z) d z=2 \gamma_{A} \\
& \mathcal{A}_{B B}=-\int_{0}^{\infty} \Pi_{B B}(z) d z=2 \gamma_{B} .
\end{aligned}
$$

\subsubsection{DFT description extended to solids}

The next step is to extend the density functional description to describe soft solids such as elastomers or gels. The elastic properties in such solids are of entropic origin and their structure is close to that of a liquid. Therefore, we hypothesize that these solids can be described using the Density Functional Theory in the sharp interface approximation, by replacing the repulsive pressure $p_{r}$ by an elastic stress tensor $\bar{\sigma}$. Just like the liquid in the previous section, this solid is assumed to be submitted to the long-range attractive potentials $\phi_{L S}$ and $\phi_{S S}$, which are not included in the stress tensor $\bar{\sigma}$. We therefore get a very similar expression as in the liquid case:

$$
\vec{\nabla} \cdot \bar{\sigma}-\vec{\nabla}\left(\phi_{S S}+\phi_{S L}\right)=0,
$$

as anticipated in Eq. (9.4).

The next step is to find expressions for the elements of $\vec{\Sigma}$ and $\vec{\sigma}$, which are generally not isotropic in the elastic case. In fact, it is here where the description of the solid differs from the liquid discussed in the previous section. In order to find the rheology, we split both stresses into an elastic 
part, and a reference part that is associated with some reference systen:

$$
\begin{aligned}
& \bar{\Sigma}=\bar{\Sigma}^{\mathrm{ref}}+\bar{\Sigma}^{\mathrm{el}}, \\
& \bar{\sigma}=\bar{\sigma}^{\mathrm{ref}}+\bar{\sigma}^{\mathrm{el}} .
\end{aligned}
$$

$\bar{\Sigma}^{\text {ref }}$ and $\bar{\sigma}^{\text {ref }}$ are the stresses in a reference state, which here we take as a semi-infinite solid in contact with vacuum (i.e. a solid-vacuum interface). For $\bar{\Sigma}^{\mathrm{el}}$ and $\bar{\sigma}^{\text {ref }}$ we apply Hooke's law. From here on we write the expressions from Hooke's law using the thermodynamic stress $\Sigma_{i j}^{\mathrm{el}}$ only, but the same holds for the microscopic stress $\sigma_{i j}^{\mathrm{el}}$. Hooke's law relates the components of the stress $\bar{\Sigma}^{\mathrm{el}}$ to the elastic strain $\bar{\varepsilon}$ :

$$
\bar{\Sigma}_{i j}^{\mathrm{el}}=\frac{E}{1+v}\left[\bar{\varepsilon}_{i j}+\frac{v}{1-2 v} \bar{\varepsilon}_{l l} \delta_{i j}\right]
$$

or equivalently:

$$
\bar{\varepsilon}_{i j}=\frac{1+v}{E} \Sigma_{i j}^{\mathrm{el}}-\frac{v}{E} \Sigma_{k k}^{\mathrm{el}} \delta_{i j}
$$

where

$$
\bar{\varepsilon}_{i j}=\frac{1}{2}\left(\partial_{i} u_{j}+\partial_{j} u_{i}\right),
$$

with $u_{i}$ is the displacement vector, and $E$ the Young's modulus. We consider plane strain conditions $\left(\varepsilon_{x x}=\varepsilon_{y y}=0\right.$, with the $z$-direction normal to the interface), and therefore find:

$$
\bar{\Sigma}_{x x}^{\mathrm{el}}=\frac{v}{1-v} \bar{\Sigma}_{z z}^{\mathrm{el}}
$$

As explained above, the same expression holds for $\sigma_{i j}^{\mathrm{el}}$, so:

$$
\bar{\sigma}_{x x}^{\mathrm{el}}=\frac{v}{1-v} \bar{\sigma}_{z z}^{\mathrm{el}} .
$$

This relation between the $x x$ and $z z$ components of the stress-tensor is key to the inequality between $\gamma_{S L}$ and $\mathrm{Y}_{S L}$. Note that the stress-tensor for an incompressible solid $(v=1 / 2)$ is isotropic $\left(\sigma_{x x}^{\mathrm{el}}=\sigma_{z z}^{\mathrm{el}}\right)$ and we would recover liquid behaviour: $\gamma_{S L}=\mathrm{Y}_{S L}$

The explicit forms of $\bar{\Sigma}^{\text {ref }}$ and $\bar{\sigma}^{\text {ref }}$ are not known in advance, but the relation between the two can be inferred from a force balance in the bulk which will become clear below. 
(a) $P$

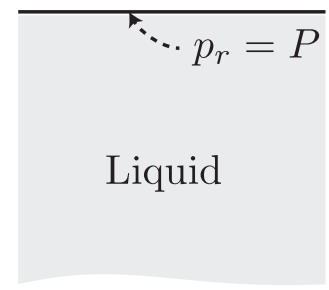

(b) $\quad P=0$ (vacuum)

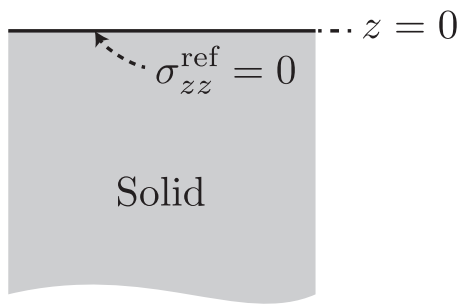

Figure 9.1: Schematic of a liquid and solid interface. (a) A liquid-vapour interface at $z=0$, where the vapour above the liquid (and thus the liquid itself as well) is at pressure $P$. Due to the low density of the vapour phase, this pressure $P$ consists entirely of short-ranged repulsive (kinetic) stress such that $p_{r}=P$. Since $p_{r}$ is continuous at the interface this can be used as a boundary condition for Eq. (9.10). (b) A solid-vacuum interface, which serves as the reference system without elastic stresses $\left(\bar{\Sigma}^{\mathrm{el}}=\bar{\sigma}^{\mathrm{el}}=0\right)$. Similarly as in the liquid, this system fixes the value of $\sigma_{z z}^{\text {ref }}=-P=0$ at $z=0$, providing the boundary condition for Eq. (9.15).

\subsubsection{Equilibrium conditions at planar interfaces}

We now consider the equilibrium near a planar interface, as sketched in Fig. 9.3. We start with the liquid. First, we note that the macroscopic equilibrium condition for a fluid reads:

$$
\Sigma_{i j}=-P \delta_{i j} \text { everywhere, }
$$

where $P$ is the thermodynamic pressure. In the liquid case we found that, cf. Eq. (9.10):

$$
p_{r}+\phi_{L L}+\phi_{S L}=\text { cnst } .
$$

The unknown constant is set by both equilibrium conditions - Eqs. (9.23) and (9.24) - at the liquid-vapour (or equivalently for a liquid-liquid) interface, Fig. 9.1(a), with the vapour phase at pressure $P$. At the liquid-vapour interface $[z=0$, cf. Fig 9.1(a)] the local potential due to liquid-liquid interactions reads $\phi_{L L}=\Pi_{L L}(0)$ and due to the absence of any solid $\phi_{S L}=0$. The vapour phase above the liquid is of such low density that only the repulsive contribution applies, and therefore we find in the vapour $p_{r}=P$. Furthermore, the stress tensor must be continuous at $z=0$, which implies $p_{r}=P$ at $z=0$. This allows us to compute the integration constant in Eq. (9.24), and 
we obtain

$$
p_{r}+\phi_{L L}+\phi_{S L}=P+\Pi_{L L}(0),
$$

everywhere within the liquid.

We now have an explicit relation between the thermodynamic pressure $P$ (or stress $\bar{\Sigma}$ ) and the microscopic contributions to the pressure $p_{r}$ and $\phi$. Later, in order to calculate the surface stress, we will require the local value of the repulsive pressure, which is simply:

$$
p_{r}=P+\Pi_{L L}(0)-\phi_{L L}-\phi_{S L} .
$$

In planar geometries, we recover that in the liquid phase $\phi_{L L}=2 \Pi_{L L}(0)-$ $\Pi_{L L}(z)$ [Fig. 9.2(a)] where the $2 \Pi_{L L}(0)$-term represents the attractive interactions due to the surrounding liquid in the bulk, and the $\Pi_{L L}(z)$-term the missing interactions (compared to the bulk) due to the presence of an interface at distance $z$. We then find, for the liquid:

$$
p_{r}=P-\Pi_{L L}(0)+\Pi_{L L}(z)-\phi_{S L},
$$

which is, in the bulk of the liquid where $\Pi_{L L}(z \rightarrow \infty)=0$ and $\phi_{S L}=0$ :

$$
p_{r}^{\text {bulk }}=P-\Pi_{L L}(0) \text {. }
$$

Finally, we derive the microscopic equilibrium equation the elastic solid. We first have to define a reference state for which the elastic stress $\bar{\sigma}^{\text {ref }}$ is zero by definition which we choose to be the state where the solid is in contact with a vacuum. We follow the same path as in the liquid case, with the distinction that in the solid case the stress tensor is not isotropic. At the solid-vacuum interface $\left[z=0\right.$, cf. Fig. 9.1(b)] we find $\sigma_{z z}^{\text {ref }}=0$, because a vacuum represents $P=0$. We use this boundary condition to determine the integration constant in Eq. (9.15), and find that:

$$
\sigma_{z z}^{\mathrm{ref}}=\Pi_{S S}(0)-\Pi_{S S}(z),
$$

where we used that $\phi_{S S}$ anywhere in the solid phase at distance $z$ from the interface is given by $2 \Pi_{S S}(0)-\Pi_{S S}(z)$, cf. Fig. 9.2(a). Furthermore, since $\nabla \cdot \bar{\Sigma}=0$ and $\Sigma_{z z}(z=0)=0$ we find:

$$
\sum_{z z}^{\mathrm{ref}}=0 .
$$

In order to calculate the excess stress in sect. 9.3 we require a relation between $\Sigma_{x x}^{\mathrm{ref}}$ and $\sigma_{x x}^{\mathrm{ref}}$. Posing that the excess stress in the bulk is zero (which is the definition of any excess quantity), we find that in the bulk:

$$
\sigma_{x x}^{\mathrm{ref}, \mathrm{bulk}}=\Sigma_{x x}^{\mathrm{ref}}+\Pi_{S S}(0) \text {. }
$$


(a)

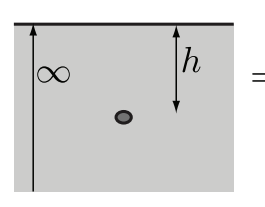

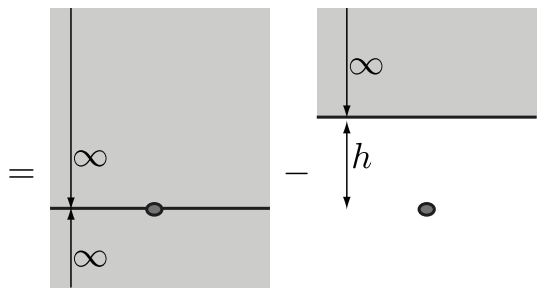

(b)

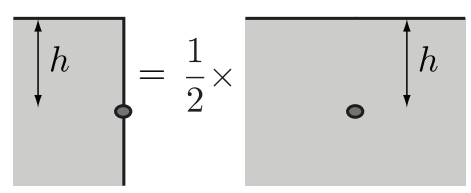

Figure 9.2: Schematics on the construction of the integration domains required to evaluate the potential on the edge of the control volume (Fig. 9.3) due to the absence of a semi-halfspace of material $\alpha$ at distance $h$. (a) The potential at the indicated point in the condensed phase (at distance $h$ from the vacuum interface) is constructed by subtracting the potential due to a semi-infinite volume of the condensed phase at distance $h\left[\Pi_{\alpha \alpha}(h)\right]$ from the potential of a complete, infinite volume of the condensed phase $\left[2 \Pi_{\alpha \alpha}(0)\right]$. (b) To evaluate the potential at the edge of the control volume as indicated in Fig. 9.3, we only take into account the potential due to material outside this control volume, which is a quarter-space, thus only half of the result from (a). Finally, one obtains $\Pi_{\alpha \alpha}(0)-\Pi_{\alpha \alpha}(h) / 2$.

Which is the solid analogue of (9.28) for the liquid. Although the bulk value of $\bar{\sigma}_{x x}^{\text {ref }}$ is now known, we did not yet account for the presence of the vacuum, which is felt at small distances from an interface. Analogous to the liquid in Eq. (9.27) this will give:

$$
\sigma_{x x}^{\mathrm{ref}}=\Sigma_{x x}^{\mathrm{ref}}+\Pi_{S S}(0)-\Pi_{S S}(z) .
$$

Note that the addition of the term $-\Pi_{S S}(z)$ does not invalidate the stressvalue we recovered in the bulk, Eq. (9.31), as for large $z$ this term becomes zero. We also anticipate that we do not require an explicit expression for $\sum_{x x}^{\text {ref }}$, as it will cancel out when we compute the excess stress as described in the next section.

\subsection{Excess quantities and surface stress}

In this section we describe how to derive the surface stress at an interface, by calculating the total excess stress at such an interface. Introducing the coordinate $z$ normal to the interface the interfacial excess quantity of an extensive measure $M$ is defined as

$$
\mathcal{M}_{A B}=S_{A B} \int\left[m(z)-m^{A} \theta(z)-m^{B} \theta(-z)\right] d z,
$$


with $m(z)$ the corresponding intensive quantity and $\theta$ the Heaviside stepfunction. The total excess $\mathcal{M}$ is therefore the (integrated) difference between the bulk values of $m^{A}$ and $m^{B}$ and the true value of $m(z)$ that varies continuously in the vicinity of the interface. Throughout the analysis we will use superscripts to denote the bulk value of a given phase. Thermodynamic quantities such as mass density, entropy or energy exhibit an interfacial excess. For example, the surface free energy $\gamma_{A B}$ can be evaluated from the microscopic interactions using the classical relations (9.12-9.14).

In this section we calculate the surface stress $\mathrm{Y}_{A B}$, which is depicted schematically in Fig. 9.3. The surface stress is defined as the intregrated excess stress, and has the dimension force per unit length, or equivalently energy per unit area. While for liquid-vapor or liquid-liquid interfaces the surface stress $Y_{A B}$ is identical to the surface free energy $\gamma_{A B}$ (following from the virtual work principle), this is not the case when one of the phases is a solid. In this section we provide explicit expressions for $Y_{A B}$, starting from the definition

$$
F_{x}=\mathrm{Y}_{A B}+\int_{0}^{\infty} d z \Sigma_{x x}^{A}+\int_{-\infty}^{0} d z \Sigma_{x x}^{B}
$$

where $\Sigma_{i j}^{A}$ and $\Sigma_{i j}^{B}$ are thermodynamic bulk stresses; note that these thermodynamics stresses implicitly contain all microscopic interactions (short- and long-ranged), so that for the liquid one simply recovers the thermodynamic pressure $\Sigma_{i j}=-P \delta_{i j}$. $F_{x}$ is the total force per unit length acting on the control volume $A B$, left of the dashed line in Fig. 9.3, projected parallel to the interface. This force is exerted by the volume indicated $A^{\prime} B^{\prime}$, right of the dashed line in Fig. 9.3. By computing this force $F_{x}$ explicitly from the microscopic models described in the previous section, we will obtain explicit expressions for $\mathrm{Y}_{A B}$ for all combinations of liquid/vapour, liquid/liquid, and solid/liquid interfaces.

\subsubsection{Force calculation}

The interactions in the DFT models consist of a short-ranged contact stress $\sigma_{i j}$, and long-ranged potential interactions $\phi_{\alpha \beta}$. The total mechanical force on the control volume therefore reads 


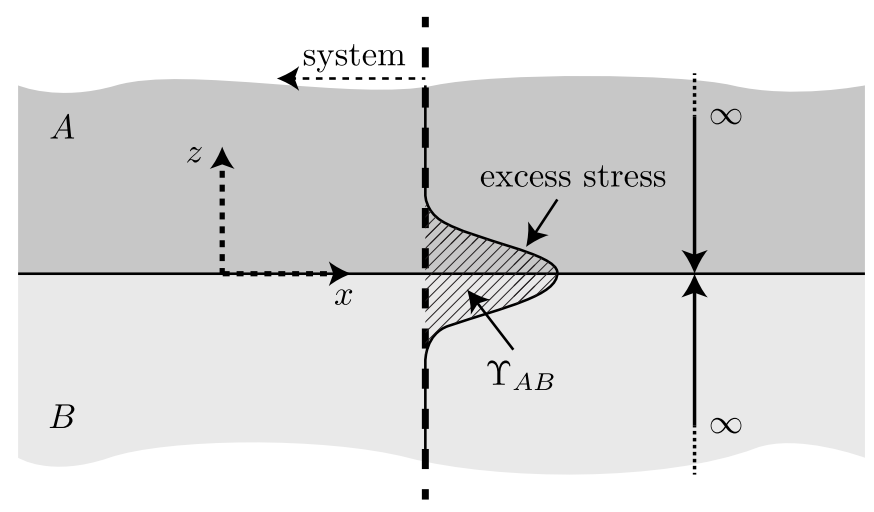

Figure 9.3: Schematic of a planar interface between two semi-infinite phases $A$ and $B$. Near the interface an excess stress develops and the total (integrated) excess stress is defined as the surface stress $\mathrm{Y}_{A B}$. Note that, by definition, the excess stress is zero in the bulk (far away from the interface). To evaluate the surface stress associated with this interface $\mathrm{Y}_{A B}$ we define a control surface perpendicular to the $A B$-interface, across which the excess stress on the control volume (left) due to contact forces and long-range forces originating from outside the control volume is integrated.

$$
\begin{aligned}
F_{x}= & \int_{0}^{\infty} d z\left(\sigma_{x x}-\phi_{A^{\prime} A}-\phi_{B^{\prime} A}\right) \\
& +\int_{-\infty}^{0} d z\left(\sigma_{x x}-\phi_{B^{\prime} B}-\phi_{A^{\prime} B}\right),
\end{aligned}
$$

where we integrated the the long-ranged forces per unit volume, $-\partial \phi_{\alpha \beta} / \partial x$, up to the dashed line in Fig. 9.3. The terms $\phi_{A^{\prime} A}$ represents the potential induced by volume $A^{\prime}$ (outside the control volume) on the part of the same phase that lies within the control volume. Note the subtle difference with $\phi_{A A}$ appearing in the equilibrium for $p_{r}$ which is the potential energy due to the entire volume. Combined with the definition (9.34), we find the surface stress: 


$$
\begin{aligned}
\mathrm{Y}_{A B}= & \int_{0}^{\infty} d z\left(\sigma_{x x}-\phi_{A^{\prime} A}-\Sigma_{x x}^{A}\right) \\
& +\int_{-\infty}^{0} d z\left(\sigma_{x x}-\phi_{B^{\prime} B}-\Sigma_{x x}^{B}\right) \\
& -\int_{0}^{\infty} d z \phi_{B^{\prime} A}-\int_{-\infty}^{0} d z \phi_{A^{\prime} B} .
\end{aligned}
$$

As the long-ranged potentials $\phi_{\alpha \beta}$ follow from volume integrals [see Eq. (9.8)], their contribution is completely independent of the rheology of the materials and can be readily evaluated. In addition, all the energies can be expressed directly in terms of $\Pi_{\alpha \beta}$, for which phase $\alpha$ is a flat semi-infinite space, owing to the symmetry of the domains in Fig. 9.3. Let us first exploit this connection for the "cross terms" $B^{\prime} A$ and $A^{\prime} B$. From the geometry one can see that $\phi_{B^{\prime} A}=\frac{1}{2} \Pi_{B A}$, due to a missing quadrant of phase $B$. Since by symmetry $\phi_{A^{\prime} B}=\frac{1}{2} \Pi_{A B}=\frac{1}{2} \Pi_{B A}$, the last two terms in (9.36) combine to an integral over $\Pi_{A B}$ and according to (9.12) this yields the adhesion energy $\mathcal{A}_{A B}=\gamma_{A}+\gamma_{B}-\gamma_{A B}$. The terms $A^{\prime} A$ and $B^{\prime} B$ are slightly more difficult to interpret, as can be seen from Fig. 9.2. As explained in detail in the caption of the figure, one finds $\phi_{A^{\prime} A}=\frac{1}{2}\left(2 \Pi_{A A}(0)-\Pi_{A A}(z)\right)$.

Using the above expressions for the potentials, we can split the surface stress (9.36) into three contributions:

$$
\mathrm{Y}_{A B}=\mathrm{Y}_{A B}^{A}+\mathrm{Y}_{A B}^{B}+\left(\gamma_{A}+\gamma_{B}-\gamma_{A B}\right)
$$

where

$$
\begin{aligned}
Y_{A B}^{A} & = & & \int_{0}^{\infty} d z\left(\sigma_{x x}-\Pi_{A A}(0)+\frac{1}{2} \Pi_{A A}(z)-\Sigma_{x x}^{A}\right), \\
Y_{A B}^{B} & = & & \int_{-\infty}^{0} d z\left(\sigma_{x x}-\Pi_{B B}(0)+\frac{1}{2} \Pi_{B B}(-z)-\Sigma_{x x}^{B}\right) \\
& = & & \int_{0}^{\infty} d z\left(\sigma_{x x}-\Pi_{B B}(0)+\frac{1}{2} \Pi_{B B}(z)-\Sigma_{x x}^{B}\right) .
\end{aligned}
$$

The last contribution of (9.37) is the work of adhesion; it is completely independent of the rheological properties of phases $A$ and $B$. By contrast, the direct interactions $\mathrm{Y}_{A B}^{A}$ and $\mathrm{Y}_{A B}^{B}$ involve the stress $\sigma_{x x}$, which has to be solved from the rheology as will be described below. 


\subsubsection{The liquid/vapor interface: $\mathrm{Y}_{L V}=\gamma_{L V}$}

From the thermodynamic relation by Shuttleworth it follows that for incompressible liquids there is a strict equality between surface energy and surface stress $[34,40]$. Hence, one usually does not distinguish between the two concepts, and simply use the nomenclature of surface tension. As a consistency check for the formalism that is developed in this paper, we first verify explicitly that this equality is recovered for a liquid/vapor interface, i.e. $\mathrm{Y}_{L V}=\gamma_{L V}$ and a liquid/liquid interface, i.e. $\mathrm{Y}_{A B}=\gamma_{A B}$ with $A, B$ liquids. Then, we will study the particular case of a liquid in contact with an elastic solid, for which we will see that in general $\mathrm{Y}_{S L} \neq \gamma_{S L}$.

Let us therefore consider phase $A$ to be vacuum and phase $B$ to be a liquid. The thermodynamic stress inside the liquid is isotropic, i.e. $\Sigma_{x x}^{B}=$ $-P$. For a true vacuum the thermodynamic pressure is strictly zero, but in real physical systems the liquid will equilibrate at a small nonzero vapor pressure. For such a liquid/vapor interface, the surface energies become $\gamma_{A}=0, \gamma_{B}=\gamma_{L V}$ and $\gamma_{A B}=\gamma_{L V}$. As a consequence of the vacuum, the work of adhesion $\gamma_{A}+\gamma_{B}-\gamma_{A B}=0$, and also $Y_{A B}^{A}=0$. Hence, the total surface stress $\mathrm{Y}_{L V}$ only receives a contribution from liquid-liquid interactions, $\mathrm{Y}_{L V}=$ $Y_{L V}^{L}$, as defined in (9.38).

The integral $(9.38 \mathrm{~b})$ involves $\sigma_{x x}$, which from Eqs. $(9.24,9.27)$ can be written

$$
\begin{aligned}
\sigma_{x x} & =-p_{r}=-P-\Pi_{L L}(0)+\phi_{L L} \\
& =-P+\Pi_{L L}(0)-\Pi_{L L}(-z) .
\end{aligned}
$$

Inserting this in (9.38b), one finds a simplified expression

$$
Y_{L V}^{V}=-\frac{1}{2} \int_{-\infty}^{0} d z \Pi_{L L}(-z)=-\frac{1}{2} \int_{0}^{\infty} d z \Pi_{L L}(z) .
$$

According to (9.13), the last integral can indeed be identified with the surface energy $\gamma_{L V}$. This completes the demonstration that the mechanical excess stress at the liquid/vapor interface, defined in Fig. 9.3 and computed from the DFT model, is strictly equal to the surface energy: $\mathrm{Y}_{L V}=\gamma_{L V}$.

\subsubsection{The liquid/liquid interface: $\mathrm{Y}_{A B}=\gamma_{A B}$}

We now consider an interface between two immiscible liquids $A$ and $B$, so again the stress tensor will be isotropic $\Sigma_{x x}^{A}=\Sigma_{x x}^{B}=-P$. Retracing the steps 
of the analyis for the liquid/vapor interface, the main difference is that the equilibrium equation for the repulsive pressure $p_{r}$ now has a contribution $\phi_{A B}=\Pi_{A B}$. Hence, one finds in phase $A$ :

$$
\sigma_{x x}=-p_{r}=-P+\Pi_{A A}(0)-\Pi_{A A}(z)+\Pi_{A B}(z),
$$

while in phase $B$

$$
\sigma_{x x}=-p_{r}=-P+\Pi_{B B}(0)-\Pi_{B B}(z)+\Pi_{A B}(-z) .
$$

Note that this result is equivalent to Eq. (9.27), with the solid phase replaced by the other liquid. This gives for the integrals (9.38)

$$
\begin{aligned}
& Y_{A B}^{A}=\gamma_{A}-\left(\gamma_{A}+\gamma_{B}-\gamma_{A B}\right)=\gamma_{A B}-\gamma_{B}, \\
& Y_{A B}^{B}=\gamma_{B}-\left(\gamma_{A}+\gamma_{B}-\gamma_{A B}\right)=\gamma_{A B}-\gamma_{A} .
\end{aligned}
$$

Adding the work of adhesion, one indeed obtains that the surface stress is equal to the surface energy:

$$
\mathrm{Y}_{A B}=\mathrm{Y}_{A B}^{A}+\mathrm{Y}_{A B}^{B}+\gamma_{A}+\gamma_{B}-\gamma_{A B}=\gamma_{A B} .
$$

\subsubsection{The solid/liquid interface}

Finally, we discuss the solid-liquid interface. As before, we evaluate the terms in the integral (9.38) seperately. The elastic properties of the solid enter through $Y_{L S}^{S}$, whereas $Y_{L S}^{L}$ is the same as that in the liquid/liquid case,

$$
Y_{L S}^{L}=\gamma_{S L}-\gamma_{S V}
$$

To obtain $\sigma_{x x}$ in the equation for $Y_{L S}^{S}$ [Eq. (9.38b)] we use once more that the normal stress $\sigma_{z z}$ is continuous across the liquid-solid inteface. At the liquid side we know that $\sigma_{z z}(z=0)=-p_{r}=-P+\Pi_{S L}(0)$, which allows us to determine the integration constant in Eq. (9.15) and we obtain, for the solid:

$$
\sigma_{z z}(z)=-P+\Pi_{S S}(0)+\Pi_{S L}(z)-\Pi_{S S}(z),
$$

To obtain the elastic response due to the presence of the liquid, we subtract the reference stress (solid in contact with vacuum, Eq. (9.29)) and obtain:

$$
\sigma_{z z}^{\mathrm{el}}(z)=\Pi_{S L}(z)-P .
$$


This is indeed the expression one would expect, as it signifies the addition of the liquid interactions $\Pi_{S L}(z)$ at pressure $P$ to the system that was defined as the reference case in Sect. 9.2.3. Then, via Hooke's law under plane strain conditions in the $x$ - and $y$-directions we get (Eq. (9.22)):

$$
\sigma_{x x}^{\mathrm{el}}(z)=\frac{v}{1-v}\left(\Pi_{S L}(z)-P\right)
$$

and thus, following Eqs. (9.17) and (9.32):

$$
\sigma_{x x}(z)=\Sigma_{x x}^{\mathrm{ref}}+\Pi_{S S}(0)-\Pi_{S S}(z)+\frac{v}{1-v}\left(\Pi_{S L}(z)-P\right) .
$$

The final step before we can evaluate $Y_{S L}^{S}$ is to determine $\Sigma_{x x}^{S}$ by applying Hooke's law on $\Sigma_{z z}^{\mathrm{el}}=-P$ :

$$
\Sigma_{x x}^{S}=\Sigma_{x x}^{\mathrm{ref}}-\frac{v}{1-v} P .
$$

We can now evaluate $Y_{S L^{\prime}}^{S}$ Eq. (9.38b), and find:

$$
\begin{aligned}
Y_{L S}^{S} & =\int_{-\infty}^{0}\left(-\frac{\Pi_{S S}(z)}{2}+\frac{v}{1-v} \Pi_{S L}(z)\right) d z \\
& =\gamma_{S V}-\frac{v}{1-v}\left(\gamma_{S V}+\gamma_{L V}-\gamma_{S L}\right),
\end{aligned}
$$

and we obtain for the total surface stress:

$$
\mathrm{Y}_{S L}=\frac{v}{1-v} \gamma_{S L}+\frac{1-2 v}{1-v}\left(\gamma_{S V}+\gamma_{L V}\right)
$$

which is the central result of this paper, Eq. (9.2).

\subsection{Discussion}

The Shuttleworth equation (9.1) provides a thermodynamic reason that one needs to distinghuish between the surface stress $Y$, i.e. the excess force per unit length in the interface, and the surface free energy $\gamma$. By computing the elastic- and enthalpic interactions from a microscopic model, we have for the first time derived an explicit relation between $\mathrm{Y}$ and $\gamma$. We find that, in general, the surface stress of a solid interface is indeed different from the surface energy of the same interface, as is quantified by Eq. (9.2). It turns out that the difference between surface stress and surface energy depends 


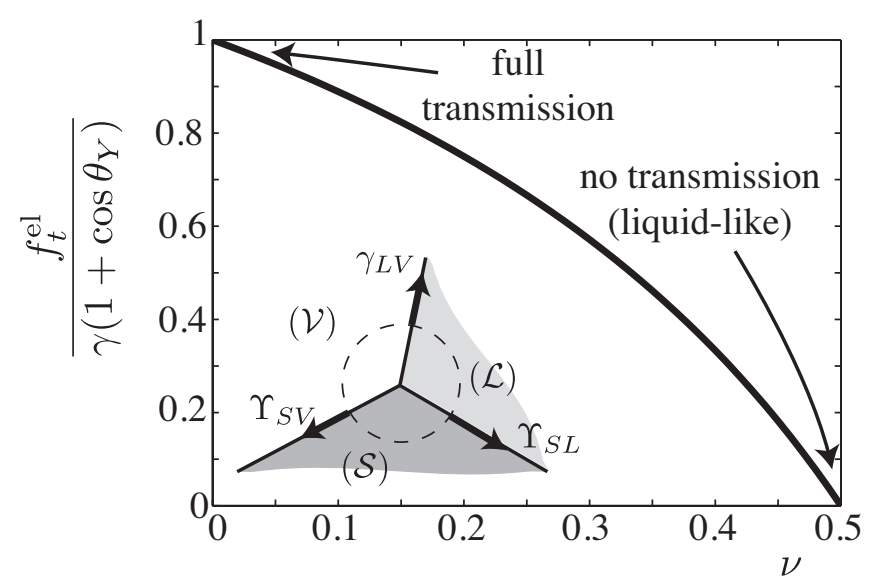

Figure 9.4: Dependence of the transmitted tangential stress on $v$ : Eq. (9.52). For $v=$ 0 the full stress on the solid induced by the presence of the contact line is transmitted to the solid bulk, where it is countered by elastic effects. For incompressible solids, and liquids, $v=1 / 2$. In this case, not tangential stress is transmitted. This must necessarily hold for liquids, which can offer no elastic resistance to any transmitted tangential stress.

on the compressibility of the phases through Poisson's ratio $v$. The relation recovers that for incompressible solids $(v=1 / 2)$ the surface stress is equal to the surface energy, in line with the fact that for incompressible liquids $\gamma=\mathrm{Y}$. Also, the model predicts an equality $\gamma_{S V}=\mathrm{Y}_{S V}$ for a solid-vapor interface of arbitrary value of $v$ [consider (9.2) with $L=V$ and $\gamma_{V V}=0$ ].

In our previous work [40] we have shown that the difference between surface stress and surface energy is crucial for understanding the elastic deformation of partially wetted deformable surfaces. In particular, it was demonstrated that the contact line region transmits a residual tangential force to the substrate, which has to be balanced by bulk elasticity. The magnitude of this tangential force can be expressed as

$$
f_{t}^{e l}=\left(\mathrm{Y}_{S L}-\mathrm{Y}_{S V}\right)-\left(\gamma_{S L}-\gamma_{S V}\right)
$$

With (9.2) this becomes:

$$
f_{t}^{e l}=\frac{1-2 v}{1-v} \gamma(1+\cos \theta) .
$$

The key feature of this result is that a finite tangential force is transmit- 
ted whenever the Poisson ratio $v \neq 1 / 2$, see Fig. 9.4. The influence of such a tangential force is usually not taken into account when describing elastocapillary deformations $[9-28,30-33,41]$. One might argue that for most experiments this is a valid assumption: soft rubbers and gels are essentially incompressible, in which case our model recovers $f_{t}^{e l}=0$. Intriguingly, however, recent experiments on a soft elastomeric wires clearly demonstrate that a tangential force is transmitted across the surface, and give rise to a significant tangential bulk strain [42]. This strongly suggests that, while the bulk material clearly has $v \approx 1 / 2$, the interfacial layers of soft elastomers do exhibit a substantial degree of compressibility. This would explain why a tangential stress is transmitted in the vicinity of the contact line, even for incompressible bulk materials. A final consequence of such a tangential force is that the contact angles on very soft surfaces do not follow Neumann's law [43]. This provides a strong limitation on the recently proposed method to determine surface stresses from contact angle measurements [44]. Our work clearly shows that a more detailed microscopic description of the interfacial chemistry, combined with further experimental characterizations, are necessary to fully resolve the physics of elastocapillarity.

\section{References}

[1] C. Bach, M. Giesen, H. Ibach, and T. Einstein, Stress relief in reconstruction, Phys. Rev. Lett. 78, 4225 (1997).

[2] A. Filippetti and V. Fiorentini, Reconstructions of $\operatorname{Ir}(110)$ and (100): An ab initio study, Surf. Sci. 377, 112 (1997).

[3] P. Wynblatt and R. Ku, Surface-energy and solute strain-energy effects in surface segregation, Surf. Sci. 65, 511 (1977).

[4] H. Ibach, The relation between the strain-dependence of the heat of adsorption and the coverage dependence of the adsorbate induced surface stress, Surf. Sci. 556, 71 (2004).

[5] P. Muller and A. Saul, Elastic effects on surface physics, Surf. Sci. Rep. 54, 157 (2004).

[6] O. Alerhand, D. Vanderbilt, R. Maede, and J. Joannopoulos, Spontaneous formation of stress domains on crystal-surfaces, Phys. Rev. Lett. 61, 1973 (1988). 
[7] J. Metois, A. Saul, and P. Muller, Measuring the surface stress polar dependence, Nat. Mater. 4, 238 (2005).

[8] E. Ramé, Encyclopedia of Surface and Colloid Science (Marcel Dekker, New York, 2002), pp. 3602-3618.

[9] J. Bico, B. Roman, L. Moulin, and A. Boudaoud, Elastocapillary coalescence in wet hair, Nature 432, 690 (2004).

[10] C. Py, P. Reverdy, L. Doppler, J. Bico, B. Roman, and C. N. Baroud, Capillary Origami: Spontaneous wrapping of a droplet with an elastic sheet, Phys. Rev. Lett. 98, 156103 (2007).

[11] A. Boudaoud, J. Bico, and B. Roman, Elastocapillary coalescence: aggregation and fragmentation with a maximal size, Phys. Rev. E 76, 060102 (2007).

[12] C. Py, P. Reverdy, L. Doppler, J. Bico, B. Roman, and C. N. Baroud, Capillarity induced folding of elastic sheets, Eur. Phys. J. Spec. Top. 166, 67 (2009).

[13] J. W. van Honschoten, J. W. Berenschot, T. Ondarcuhu, R. G. P. Sanders, J. Sundaram, M. Elwenspoek, and N. R. Tas, Elastocapillary fabrication of three-dimensional microstructures, Appl. Phys. Lett. 97, 014103 (2010).

[14] B. Roman and J. Bico, Elasto-capillarity: deforming an elastic structure with a liquid droplet, J. Phys. Cond. Matt. 22, 493101 (2010).

[15] F. Chiodi, B. Roman, and J. Bico, Piercing an interface with a brush: collaborative stiffening, Europhys. Lett. 90, 44006 (2010).

[16] J. Hure, B. Roman, and J. Bico, Wrapping an adhesive sphere with an elastic sheet, J. Phys. Chem. B 106, 174301 (2011).

[17] G. Lester, Contact angles of liquids at deformable solid surfaces, J. Colloid Sci. 16, 315 (1961).

[18] A. Rusanov, Theory of wetting of elastically deformed bodies .1. Deformation with a finite contact-angle, Colloid J. USSR 37, 614 (1975).

[19] S. Yuk and M. Jhon, Contact angles on deformable solids, J. Colloid Interface Sci. 110, 252 (1986).

[20] M. Shanahan, The influence of solid micro-deformation on contact-angle equilibrium, J. Phys. D-Appl. Phys. 20, 945 (1987). 
[21] A. Carre, J. Gastel, and M. Shanahan, Viscoelastic effects in the spreading of liquids, Nature 379, 432 (1996).

[22] L. White, The contact angle on an elastic substrate. 1 . The role of disjoining pressure in the surface mechanics, J. Colloid Interface Sci. 258, 82 (2003).

[23] R. Pericet-Camara, E. Bonaccurso, and K. Graf, Microstructuring of polystyrene surfaces with nonsolvent sessile droplets, ChemPhysChem 9, 1738 (2008).

[24] R. Pericet-Camara, A. Best, H.-J. Butt, and E. Bonaccurso, Effect of capillary pressure and surface tension on the deformation of elastic surfaces by sessile liquid microdrops: An experimental investigation, Langmuir 24, 10565 (2008).

[25] S. Mora, T. Phou, J.-M. Fromental, L. M. Pismen, and Y. Pomeau, Capillarity Driven Instability of a Soft Solid, Phys. Rev. Lett. 105, 214301 (2010).

[26] M. Sokuler, G. K. Auernhammer, M. Roth, C. Liu, E. Bonaccurso, and H.-J. Butt, The Softer the Better: Fast Condensation on Soft Surfaces, Langmuir 26, 1544 (2010).

[27] F. Leonforte and M. Mueller, Statics of polymer droplets on deformable surfaces, J. Chem. Phys. 135, 214703 (2011).

[28] E. R. Jerison, Y. Xu, L. A. Wilen, and E. R. Dufresne, Deformation of an Elastic Substrate by a Three-Phase Contact Line, Phys. Rev. Lett. 106, 186103 (2011).

[29] S. Das, A. Marchand, B. Andreotti, and J. H. Snoeijer, Elastic deformation due to tangential capillary forces, Phys. Fluids 23, 072006 (2011).

[30] R. W. Style, R. Boltyanskiy, Y. Che, J. S. Wettlaufer, L. A. Wilen, and E. R. Dufresne, Universal Deformation of Soft Substrates Near a Contact Line and the Direct Measurement of Solid Surface Stresses, Phys. Rev. Lett. 110, 066103 (2013).

[31] Y. Wang, B. Bhushan, and X. Zhao, Nanoindents produced by nanobubbles on ultrathin polystyrene films in water, Nanotechnology 20, 045301 (2009).

[32] R. W. Style and E. R. Dufresne, Static wetting on deformable substrates, from liquids to soft solids, Soft Matter 8, 7177 (2012). 
[33] L. Limat, Straight contact lines on a soft, incompressible solid, Eur. Phys. J. E 35, 134 (2012).

[34] R. Shuttleworth, The surface tension of solids, Proc. Phys. Soc., London Sect. A 63, 444 (1950).

[35] J. S. Rowlinson and B. Widom, Molecular Theory of Capillarity (Clarendon, Oxford, 1982).

[36] G. J. Merchant and J. B. Keller, Contact Angles, Phys. Fluids A 4, 477 (1992).

[37] T. Getta and S. Dietrich, Line tension between fluid phases and a substrate, Phys. Rev. E 57, 655 (1998).

[38] C. Bauer and S. Dietrich, Quantitative study of laterally inhomogeneous wetting, Eur. Phys. J. B 10, 767 (1999).

[39] J.-P. Hansen and I. R. McDonald, Theory of Simple Liquids (Academic Press, Waltham, MA, 2006).

[40] J. H. Weijs, B. Andreotti, and J. Snoeijer, Elasto-capillarity at the nanoscale: on the coupling between elasticity and surface energy in soft solids, Soft Matter In press, DOI: 10.1039/C $3 S_{5} 0861 G$ (2013).

[41] C. Fradin, A. Braslau, A. Luzat, D. Smilgies, M. Alba, N. Boudet, K. R. Mecke, and J. Daillant, Reduction in the surface energy of liquid interfaces at short length scales, Nature 403, 871 (2000).

[42] A. Marchand, S. Das, J. H. Snoeijer, and B. Andreotti, Capillary Pressure and Contact Line Force on a Soft Solid, Phys. Rev. Lett. 108, 094301 (2012).

[43] A. Marchand, S. Das, J. H. Snoeijer, and B. Andreotti, Contact Angles on a Soft Solid: From Young's Law to Neumann's Law, Phys. Rev. Lett. 109, 236101 (2012).

[44] R. W. Style, R. Boltyanskiy, Y. Che, J. S. Wettlaufer, L. A. Wilen, and E. R. Dufresne, Universal Deformation of Soft Substrates Near a Contact Line and the Direct Measurement of Solid Surface Stresses, Phys. Rev. Lett. 110, 066103 (2013). 


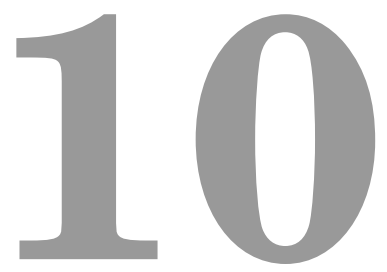

\section{Initial spreading of low-viscosity drops on partially wetting surfaces * +}

Liquid drops start spreading directly after brought into contact with a partial wetting substrate. Although this phenomenon involves a three-phase contact line, the spreading motion is very fast. We study the initial spreading dynamics of lowviscosity drops, using two complementary methods: Molecular Dynamics simulations and high-speed imaging. We access previously unexplored length- and timescales, and provide a detailed picture on how the initial contact between the liquid drop and the solid is established. Both methods unambiguously point towards a spreading regime that is independent of wettability, with the contact radius growing as the square root of time.

\subsection{Introduction}

How fast can a liquid drop spread over a surface? This basic question is relevant for applications ranging from printing and coating, to agricultural

\footnotetext{
*Published as: K.G. Winkels, J.H. Weijs, A. Eddi, and J.H. Snoeijer, "Initial spreading of low-viscosity drops on partially wetting surfaces", Phys. Rev. E 85, 055301(R) (2012).

${ }^{\dagger}$ The numerical simulations in this chapter are part of the present thesis. The experimental work was done by K.G. Winkels and A. Eddi.
} 
applications [1-5]. In the final stage of drop spreading the dynamics are governed by Tanner's law, which relates the radius of the wetted area with time as $r \sim t^{1 / 10}[6,7]$. This extremely slow dynamics emerges from a balance between surface tension and viscous forces close to the contact line [3]. Much less is known about the early stages of spreading, just after a spherical drop is brought into contact with a substrate at vanishing approach velocity. In contrast to Tanner's law, this dynamics is very fast [8-12]: capillary energy suddenly becomes available when the drop touches the solid, and this energy is concentrated into a singular point of contact. It has remained unclear whether or not the wetting conditions can influence such rapid inertial flows [13-15].

The initial stages of drop spreading are strongly reminiscent of the coalescence of two spherical drops of liquid, which very rapidly merge after contact is established [16-21]. For low-viscosity liquids such as water, it is well-known that the contact area between the drops grows as $r \sim t^{1 / 2}$ during coalescence. This can be explained from the balance of the inertial pressure inside the drop, $\sim \rho(d r / d t)^{2}$, and the capillary pressure, $\sim \gamma R / r^{2}$. Here $\rho$ is the density, $\gamma$ the surface tension, and $R$ the drop radius. Interestingly, an identical scaling law was observed experimentally for water drops spreading on a completely wetting surface [8]; apparently, the presence of a three-phase contact line does not affect the pressure balance during the initial phase of spreading. A rather different picture emerged, however, for drops spreading on partially wetting surfaces [9-11]. The dynamics was found to depend strongly on surface wettability, $r \sim t^{\alpha}$, with a non-universal exponent $\alpha$ that varies with the equilibrium contact angle [9]. This raises a number of intriguing questions: How can the contact line, and the surface chemistry, affect the "coalescence" of a drop with a surface? Are the initial stages of spreading truly non-universal, or is there a hidden regime at smaller times? How is contact established on a molecular scale?

In this chapter we reveal the initial spreading dynamics of low-viscosity drops, using two complementary methods: Molecular Dynamics simulations of Lennard-Jones nanodrops and high-speed imaging of experiments on millimeter-sized water drops [Fig. 10.1]. We access previously unexplored length- and time-scales, and provide a detailed picture on how the initial contact between the liquid drop and the solid is established. While simulations and experiments describe different dynamical regimes, both methods unambiguously point towards a universal spreading regime independent of wettability, consistent with the inertia-capillary balance $r \sim t^{1 / 2}$. 

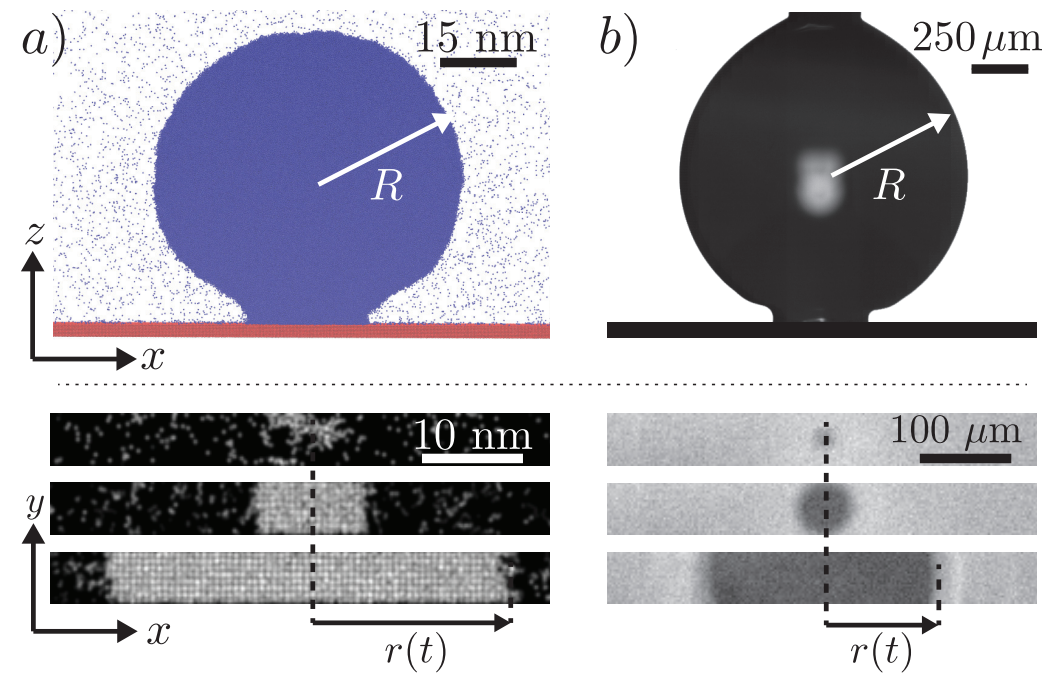

Figure 10.1: Initial stages of drop spreading on partially wetting surfaces of varying wettability. (a) Molecular Dynamics simulations of Lennard-Jones nanodrops $(R=$ $30 \mathrm{~nm})$, and (b) Experiments of water drops $(R=0.5 \mathrm{~mm})$. The top panels show side views of the liquid drop just after it has made contact with the partially wetting substrate. Lower panels are bottom views at times (a) $t=10,35,400$ ps, and (b) $t=4,8,44 \mu \mathrm{s}$. The contact radius $r(t)$ can be measured in time.

This contrasts the scenario proposed by [9]: At very early times after contact, the spreading exponent is independent of wettability, for contact angles ranging from complete wetting to very hydrophobic.

\subsection{Molecular Dynamics simulations}

The use of Molecular Dynamics simulations (MD) allows for studying the initial contact between a liquid drop and a solid substrate down to molecular scale. To reveal the fundamental mechanism of contact and subsequent spreading, we use a generic Lennard-Jones liquid. The advantage of the molecular approach is that, unlike continuum modeling, no assumptions on the moving contact line singularity [3] are needed. In MD, the wetting characteristics are directly controlled by the solid-liquid interaction, which determines the equilibrium contact angle $\theta_{e q}$, see also chapter 7 . The challenge, however, is to achieve sufficiently large drop sizes to recover a hy- 
drodynamic regime. We therefore study a quasi-two-dimensional geometry rather than axisymmetric drops, in which the system size in the $y$-direction is only 15 molecular sizes [cf. Fig. 10.1(b)]. Indeed, contact problems such as coalescence are known to be essentially $2 \mathrm{D}$ phenomena and the same is expected here [16, 22].

We perform simulations on binary systems, in which two types of particles exist: fluid particles that can move around either in the gas or liquid phase, and solid particles which are frozen on an fcc-lattice and constitute the solid substrate [23]. All particle interactions are defined by the LennardJones potential:

$$
\phi_{i j}(r)=4 \epsilon_{i j}\left[\left(\frac{\sigma_{i j}}{r}\right)^{12}-\left(\frac{\sigma_{i j}}{r}\right)^{6}\right] .
$$

Here, $\epsilon_{i j}$ is the interaction strength between particles $i$ and $j$ and $\sigma_{i j}$ the characteristic size of the atoms. This size is the same for all interactions, $\sigma_{i j}=\sigma=0.34 \mathrm{~nm}$. The potential function is truncated at $r_{c}=5 \sigma(1.7 \mathrm{~nm})$ where $\phi_{i j}$ is practically zero. The mass of the atoms was set at $20 \mathrm{amu}$, and a timestep of $1.75 \mathrm{fs}$ was found to be sufficient to accurately model these systems. The interaction strengths between the fluid atoms are $\epsilon_{l l}=1.2 k_{B} T$, with $k_{B}$ the Boltzmann constant and $T$ the temperature. The simulations are done in the NVT-ensemble, where the temperature is held at $300 \mathrm{~K}$ using a thermostat, which is below the critical point for a Lennard-Jones fluid with the interaction strengths used. The fluid particles (amount: $\left.N_{l}=304,192\right)$ are initially positioned on an fcc-lattice (shaped with a cubic outline) far from the substrate $\left(N_{s}=78,300\right)$, but are free to move around and relax towards an equilibrium drop shape. Periodic boundary conditions are present in the lateral directions. The dimensions of the quasi-2D system are $240 \mathrm{~nm}, 5.1 \mathrm{~nm}$ and $120 \mathrm{~nm}$ in the $x-, y$ - and $z$-directions respectively (Fig. 10.1). The depth of the system is short enough to suppress the Rayleigh-Plateau instability and leads to an infinitely long cylindrical-cap shaped drop.

The interaction strength between the solid and fluid defines the contact angle [24]. We considered four different wettabilities (thus, four different values of $\left.\epsilon_{s l}\right): \epsilon_{s l}=(0.3,0.4,0.8,1.2) k_{B} T$, giving $\theta_{e q}=\left(115^{\circ}, 100^{\circ}, 60^{\circ}, 0^{\circ}\right) \pm$ $10^{\circ}$. The liquid density was measured to be $\rho=664 \mathrm{~kg} / \mathrm{m}^{3}$, and the surface tension $\gamma$ was measured in a separate, planar system at $\gamma=0.017 \mathrm{~J} / \mathrm{m}^{2}$. The viscosity of the liquid was measured in another separate system (Poiseuillegeometry) to be $\eta=3.64 \cdot 10^{-4} \mathrm{~kg} /(\mathrm{m} \cdot \mathrm{s})$.

The following procedure was used to bring the drop into contact with the 
a)
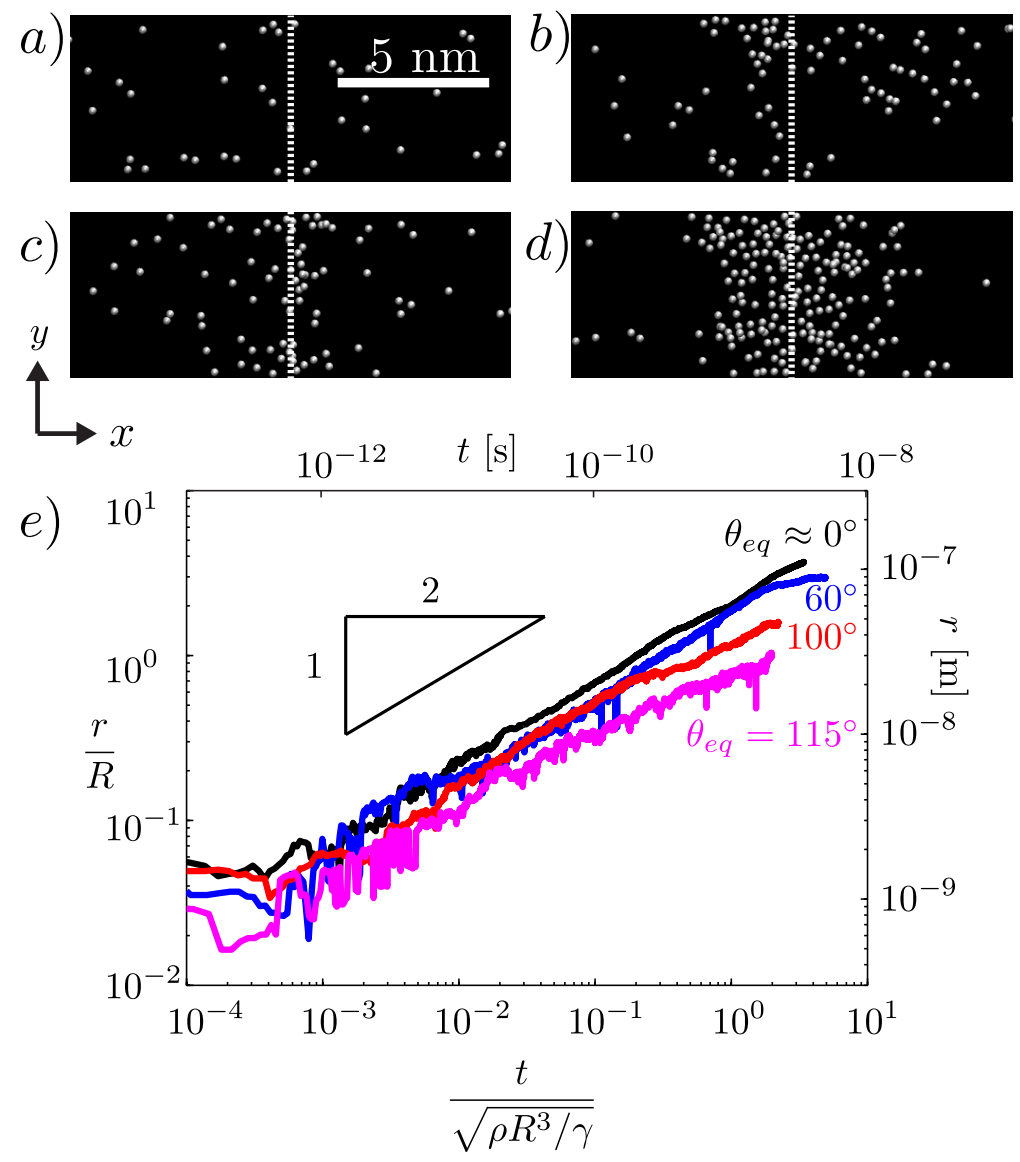

Figure 10.2: Molecular Dynamics results. (a-d) "Bottom views" showing the molecules within $0.5 \mathrm{~nm}$ from the substrate just prior to and after initial contact $(t=-70 \mathrm{ps},-25 \mathrm{ps},-5 \mathrm{ps}$ and $35 \mathrm{ps}$, respectively). (a) A small number of molecules from the vapor phase is close to the substrate. $(\mathrm{b}, \mathrm{c})$ Some fluctuating patches of higher density form. (d) A region of high liquid density nucleates at the substrate, from which one can measure $r(t)$. (e) Radius of wetted area as a function of time for varying substrate wettabilities $\theta_{e q}$. Once the contact is established, we observe a power-law with exponent $1 / 2$ for all values of $\theta_{e q}$. Results are displayed in SI-units on the right and top axis, and displayed in dimensionless form on the left and bottom axis. Contact radius $r$ is rescaled with the initial drop radius $R$, time is rescaled with the inertial time scale $\tau_{\rho}=\sqrt{\rho R^{3} / \gamma}$. 
substrate. First, the liquid is allowed to equilibrate far away $(32 \mathrm{~nm}$ ) from the substrate. During this stage, the drop will assume its cylindrical shape $(R=30 \mathrm{~nm})$ and the liquid equilibrates with the vapour phase. Next, a body force is briefly applied on the fluid atoms until the drop moves towards the substrate. Just before the drop comes into contact with the substrate, the center-of-mass velocity of the drop is subtracted from the atom velocities such that the drop now 'hovers' above the substrate. Due to the close proximity of the substrate (around $1 \mathrm{~nm}$ ) the thermal fluctuations of the interface lead to first contact between the drop and the substrate after which the drop starts to spread. Using this method, the approach velocity of the drop towards the substrate is zero and is not a parameter in this problem.

What happens during the initial contact? In these early stages one cannot yet speak about a continuous liquid phase in contact with the solid. Instead, one first encounters the discrete, molecular nature of the fluid. Figures 10.2(a-d) show snapshots of the molecules that are within $0.5 \mathrm{~nm}$ from the substrate, represented as white dots. First a number of vapor molecules is randomly distributed over the surface [Fig. 10.2(a)]. As time progresses, more molecules come into contact and form fluctuating "patches" of high liquid density at the substrate [Figs. 10.2(b,c)]. The boundaries of these patches is extracted by computing the number density field of atoms near the surface, and taking the iso-density contour half-way between the liquid and vapor density. Eventually, the patch becomes sufficiently large to span the entire depth of the quasi-2D simulation domain, from which we define the time of contact [Fig. 2(d)]. The exact definition of $t=0$ does not influence our main conclusions below. From that moment, we track the boundaries of this wetting patch, which are the moving contact lines. The contact lines become sharper and are well-defined during the spreading, as can be seen in Fig. 10.1(a). Note that while the average vapor density close to the surface is slightly larger than in the bulk, the surface coverage is very low and does not represent a precursor film.

The key result of our MD simulations is that, once the liquid drop has established contact with the surface, the spreading follows a single power law. Figure 10.2(e) shows the contact radius $r$ versus time on surfaces with varying wettability. For all contact angles $\theta_{e q}$ we observe a scaling consistent with $r \sim t^{1 / 2}$ (best-fit exponent: 0.48). It turns out that the contact angle of the substrate $\theta_{e q}$ does have an influence on the spreading, but only through the prefactor: the exponent is always very close to $1 / 2$. The prefactor increases as the contact angle decreases, such that drops spread faster on the 
more hydrophilic surfaces.

Despite the very small length- and time-scales in these simulations, the spreading appears to be consistent with the hydrodynamic picture of inertiadominated coalescence $[16,18]$ The left axis and bottom axis in Fig. 10.2(e) represent the data in dimensionless units, where lengths are scaled with the initial drop radius $R$ and time with the inertial scale $\tau_{\rho}=\sqrt{\rho R^{3} / \gamma}$. In these units, the data span a range similar to previous experiments on millimetersized water drops [8, 9].

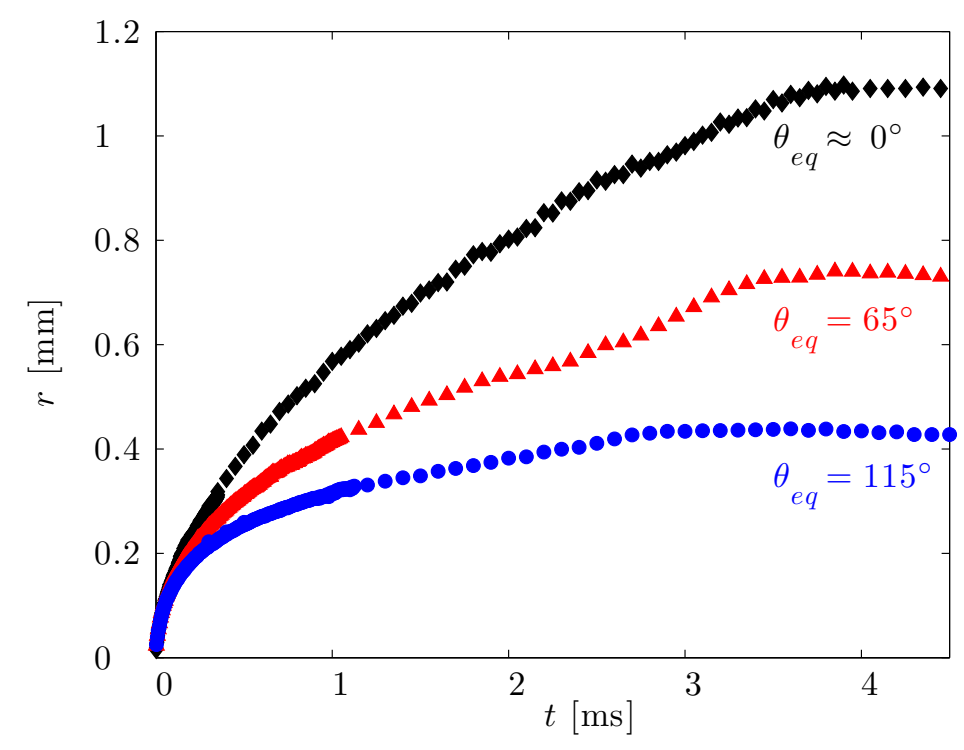

Figure 10.3: Experimental measurements of contact radius $r$ plotted as function of time $t$ for different substrate wettabilities. Results for three different equilibrium contact angles are plotted: clean glass $\left(\theta_{e} \approx 0^{\circ} ; \boldsymbol{\diamond}\right)$, coated glass $\left(\theta_{e}=65^{\circ} ; \boldsymbol{\Delta}\right)$, teflon coated glass $\left(\theta_{e}=115^{\circ} ; \mathbf{O}\right)$. The curves represent averaged data of repeated measurements (five or more for each $\theta_{e q}$ ) per substrate for drops with radius $R=0.5$ $\mathrm{mm}$, showing the reproducibility of the experiments.

\subsection{Experiments}

To verify whether the spreading behavior observed in MD is also found experimentally, we carried out experiments in a previously unexplored regime. 
The required spatial and temporal resolution is achieved by high-speed recording of drop spreading from below, using transparent substrates and recording rates up to 600,000 frames/second. Typical images are shown in Fig. 10.1b. The high-speed camera (Photron SA 1.1) is connected to a microscope (Zeiss Axiovert 25), which in combination with a 10X microscope objective (Zeiss A-plan, 10X) and reflective illumination gives a maximum resolving power of $2 \mu \mathrm{m} /$ pixel. To capture a large period of the spreading process, frame rates used are in the range of 1o-6ookfps. Quéré et al. [8] and Bird et al. [9], have shown that data for different drop sizes collapse by inertial rescaling. As here we focus on the influence of wettability, we consider only one drop radius $R=0.5 \pm 0.01 \mathrm{~mm}$.

To investigate the effect of wettability on the spreading, we performed experiments with water drops on three different substrates with different equilibrium contact angle $\theta_{e q}$ : clean glass (almost perfectly wetting, $\theta_{e q} \approx 0^{\circ}$ ), coated glass $\left(\theta_{r}=55^{\circ} ; \theta_{a}=75^{\circ} ; \theta_{e q}=65^{\circ}\right)$ and teflon coated glass $\left(\theta_{r}=110^{\circ}\right.$; $\left.\theta_{a}=120^{\circ} ; \theta_{e q}=115^{\circ}\right)$. In order to avoid any condensation effects prior to spreading, the surrounding air is saturated with nitrogen gas. A thin needle is fixed at height $D=1 \pm 0.02 \mathrm{~mm}$ above the substrate, thereby setting the initial radius $R$ of the spreading drop (height $D=2 R$ ). With a syringe pump set at a constant volume rate of $1 \mu \mathrm{l} / \mathrm{min}$ a pendant drop is grown at the needle tip, until it touches the substrate. This generates approach velocities $<2 \cdot 10^{-5} \mathrm{~m} / \mathrm{s}$, so that the outer gas dynamics has a negligible influence on the contact process. The radius of contact $r(t)$ is determined from images as in Fig. 10.1b, using a custom-made edge-detection algorithm in Matlab that finds the maximum image intensity slope in every frame.

Our experiments confirm a single power law during the initial stages of contact. The measurements of the contact radius $r(t)$ are shown in Fig. 10.3 on linear axes. One observes that the data fall onto three different curves, corresponding to the three values of $\theta_{e q}$. The curves separate about $0.1 \mathrm{~ms}$ after contact, showing a dependence on wettability at later times. However, the early-time dynamics are independent of wettability. This is revealed in Fig. 10.4, which shows the same data on log-log scale. We find that our data for different $\theta_{e q}$ perfectly collapse at early times $\left(t / \tau_{\rho}<0.04\right)$, and display an exponent close to $1 / 2$ (best fit: 0.55). We reprinted the data by Bird et al. [9] (crosses) for completeness, and find a perfect agreement with our data at $t / \tau_{\rho}>0.1$, which is the range of accurate resolution in ref. [9]. The upper/right axis represent SI-units, while for the lower/left axis we employ the inertial scaling. Thus, the key point is that our measurements reveal a 


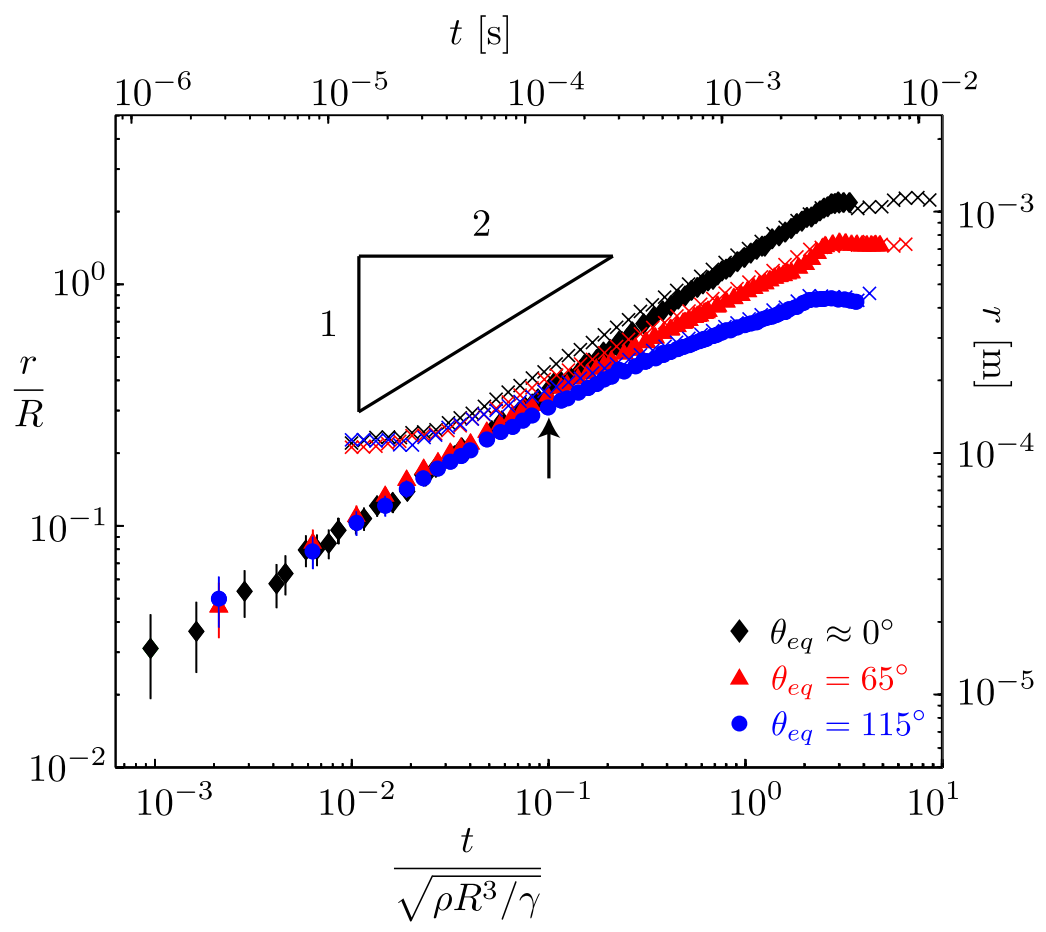

Figure 10.4: Experimental results. Contact radius $r$ measured as a function of time (top and right axis) for three different equilibrium contact angles: clean glass $\left(\theta_{e} \approx 0^{\circ} ; \diamond\right)$, coated glass $\left(\theta_{e}=65^{\circ} ; \Delta\right)$, and teflon coated glass $\left(\theta_{e}=115^{\circ} ; \bigcirc\right)$. The data shown, is an average of at least 5 measurements. The error bars denote the statistical error, which is larger than the measurement accuracy. On the left and bottom axis the data is normalized by the drop radius $R$ and inertial time $\tau_{\rho}=\sqrt{\rho R^{3} / \gamma}$ respectively. A new regime is observed at earlier times, where the spreading is independent of the equilibrium contact angle. The colored crosses are reprinted data by Bird et al. [9] (corresponding with: $\theta_{\text {eq }}=3^{\circ}, \times ; 43^{\circ}, \times$; and $117^{\circ}$, $\times)$. The arrow indicates the smallest times that were accurately resolved in the study by Bird et al.

regime where wettability has no effect on the spreading at early times, not even in the prefactor. 


\subsection{Discussion}

We have shown that early stage spreading of low-viscosity drops on a partially wetting substrate is independent of wettability. The wetted area is found to grow as $r \sim t^{1 / 2}$, for all considered wettabilities: we find no influence on the spreading exponent by the presence of a contact line. This suggests that the mechanism of capillary wave generation, invoked to explain $\theta_{e q}$-dependent spreading exponents [9], cannot be the dominant factor at very early times. Still, such capillary waves could be relevant for explaining the later stages of spreading in Fig. 10.4, where a departure from the $1 / 2$ scaling is observed. However, this departure first arises when $r / R \gtrsim 0.2$, in which case self-similarity of the bridge connecting the liquid drop and the substrate is lost, and scaling cannot be assumed a priori. It therefore remains a challenge to explain the moment when the effect of $\theta_{e q}$ becomes apparent in the experiments. There is, however, a subtle difference between the MD simulations and the experiments regarding the prefactor of the spreading law. For the experiments we observe a perfect collapse of the data on a single curve at early times. By contrast, the MD curves do not collapse, but the prefactor increases with decreasing $\theta_{\text {eq }}$. We can point out at least two possible origins for this difference. First, the time- and length-scales of the two systems differ by orders of magnitude. In addition the simulations and experiments are not dynamically similar. While the rescaled results of Fig. 10.2 and 10.4 are very close, the Reynolds numbers defined as $\operatorname{Re}=\rho r(d r / d t) / \eta$ are very different: it is order unity in MD and order 100 in experiments. This suggests that the MD could be influenced by viscous effects, and it would be interesting to further investigate spreading for highly viscous liquids $[12,21]$. Another key difference is the importance of thermal fluctuations at molecular scales. These are known to have a dramatic effect on the dynamics of drop pinch-off $[25,26]$, and it would be interesting to further explore their influence on spreading in the molecular simulations.

Finally, although wettability does not affect the initial rapid inertial flow in drop spreading, other cases are known to be strongly influenced by surface properties $[13,15,27]$. From a more general perspective, the combination of such inertial flows with a three-phase contact line therefore remains a challenge. 


\section{References}

[1] H. Wijshoff, The dynamics of the piezo inkjet printhead operation, Physics Reports 491, 77 (2010).

[2] P. Simpkins and V. Kuck, On air entrainment in coatings, J. Coll. Int. Sci. 263, 562 (2003).

[3] D. Bonn, J. Eggers, J. Indekeu, J. Meunier, and E. Rolley, Wetting and spreading, Rev. Mod. Phys. 81, 739 (2009).

[4] V. Bergeron, D. Bonn, J. Y. Martin, and L. Vovelle, Controlling droplet deposition with polymer additives, Nature 405, 6788 (2000).

[5] P. G. de Gennes, Wetting: statics and dynamics, Rev. Mod. Phys. 57, 827 (1985).

[6] L. Tanner, The spreading of silicone oil drops on horizontal surfaces, J. Phys. D: Appl. Phys. 12, 1 (1979).

[7] S. Rafaï, D. Sarker, V. Bergeron, J. Meunier, and D. Bonn, Superspreading: Aqueous Surfactant Drops Spreading on Hydrophobic Surfaces, Langmuir 18, 10486 (2002).

[8] A.-L. Biance, C. Clanet, and D. Quéré, First steps in the spreading of a liquid droplet, Phys. Rev. E 69, 016301 (2004).

[9] J. C. Bird, S. Mandre, and H. A. Stone, Short-Time Dynamics of Partial Wetting, Phys. Rev. Lett. 100, 234501 (2008).

[10] L. Courbin, J. C. Bird, M. Reyssat, and H. A. Stone, Dynamics of wetting: from inertial spreading to viscous imbibition, J. Phys.: Condens. Matter 21, 464127 (2009).

[11] A. Carlson, M. Do-Quang, and G. Amberg, Dissipation in rapid dynamic wetting, J. Fluid Mech. 682, 213 (2011).

[12] A. Carlson, G. Bellani, and G. Amberg, Universality in dynamic wetting dominated by contact-line friction, Phys. Rev. E 85, 045302(R) (2012).

[13] C. Duez, C. Ybert, C. Clanet, and L. Bocquet, Making a splash with water repellency, Nat. Phys. 3, 180 (2007). 
[14] J. Eggers, Fluid Dynamics: Coupling the large and the small, Nat. Phys. 3, 145 (2007).

[15] C. Duez, C. Ybert, C. Clanet, and L. Bocquet, Wetting Controls Separation of Inertial Flows from Solid Surfaces, Phys. Rev. Lett. 104, 084503 (2010).

[16] J. Eggers, J. R. Lister, and H. A. Stone, Coalescence of liquid drops, J. Fluid Mech. 401, 293 (1999).

[17] L. Duchemin, J. Eggers, and C. Josserand, Inviscid coalescence of drops, J. Fluid Mech. 487, 167 (2003).

[18] M. Wu, T. Cubaud, and C.-M. Ho, Scaling law in liquid drop coalescence driven by surface tension, Phys. Fluids 16, L51 (2004).

[19] S. T. Thoroddsen, K. Takehara, and T. G. Etoh, The coalescence speed of a pendent and a sessile drop, J. Fluid Mech. 527, 85 (2005).

[20] S. C. Case and S. R. Nagel, Coalescence in Low-Viscosity Liquids, Phys. Rev. Lett. 100, 084503 (2008).

[21] J. D. Paulsen, J. C. Burton, and S. R. Nagel, Viscous to Inertial Crossover in Liquid Drop Coalescence, Phys. Rev. Lett. 106, 114501 (2011).

[22] J. C. Burton and P. Taborek, Role of Dimensionality and Axisymmetry in Fluid Pinch-Off and Coalescence, Phys. Rev. Lett. 98, 224502 (2007).

[23] D. V. der Spoel, E. Lindahl, B. Hess, G. Groenhof, A. Mark, and H. Berendsen, GROMACS: Fast, flexible, and free, J. Comput. Chem. 26, 1701 (2005).

[24] J. H. Weijs, A. Marchand, B. Andreotti, D. Lohse, and J. H. Snoeijer, Origin of line tension for a Lennard-Jones nanodroplet, Phys. Fluids 23, I (2011).

[25] M. Moseler and U. Landman, Formation, Stability, and Breakup of Nanojets, Science 289, 1165 (2000).

[26] J. Eggers, Dynamics of Liquid Nanojets, Phys. Rev. Lett. 89, 084502 (2002).

[27] P. Tsai, R. C. A. van der Veen, M. van de Raa, and D. Lohse, How Micropatterns and Air Pressure Affect Splashing on Surfaces, Langmuir 26, 16090 (2010). 


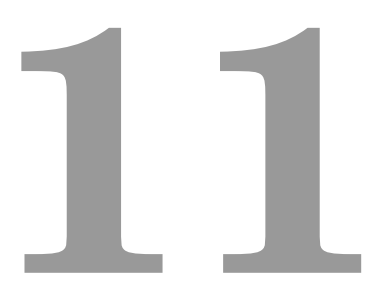

\section{Summary and Outlook}

\subsection{Summary}

In this thesis, we discussed several interfacial phenomena which can take place when a solid is brought into contact with a liquid, some examples are briefly discussed below. Surface nanobubbles can be formed, which do not seem to behave like ordinary (larger) bubbles. In particular, the lifetimes of surface nanobubbles are much longer than predicted. Also, due to capillary forces the solid gets deformed due to the presence of the liquid. The elastocapillary deformations can have a significant influence on the solid, and for slender bodies it even results in (measurable) bulk deformations. Finally, when a droplet is initially brought into contact with a solid, it will start to spread until it reached the contact angle prescribed by Young's law.

The first part of the thesis concerned surface nanobubbles. Because of the small length-scales involved, and to test down to what scale continuum modelling is applicable, we use molecular dynamics to study the formation and stability of surface nanobubbles in chapter 2. We found that in a sufficiently supersaturated liquid (this supersaturation needs only to occur locally) nanobubbles spontaneously form. Due to Brownian motion, the bubbles that form close enough to the liquid-solid surface attach to the solid and become surface nanobubbles. In the simulations, for non-pinned bub- 
bles on atomically smooth surfaces we did not recover the long lifetimes found in experiments: the nanobubbles dissolved in less than a microsecond. However, we did recover that gas-adsorbates ("micropancakes") are able to change the equilibrium contact angle of the nanobubbles drastically, and provide a model that describes this.

A natural extension of surface nanobubbles are bulk nanobubbles, i.e. nanoscopic bubbles in the bulk liquid phase. We studied these bubbles in a geometry where they are surrounded by other bulk nanobubbles at close distance, using molecular dynamics. We found that, when sufficiently close to each other, neighbouring bulk nanobubbles can shield the diffusive flux that would drain a single bulk nanobubble in less than a microsecond. This stability is theoretically explained, and using macroscopic arguments we can predict, for given dissolved gas concentration in the liquid and bubblebubble distances whether these nanobubbles are stable and what their equilibrium size is. The existence of densely packed clusters of bulk nanobubbles is in agreement with experimental work by Ohgaki et al. [1].

Bulk nanobubbles are thus well understood. Realizing that the influence of the solid must therefore be responsible for the unexplained behaviour of surface nanobubbles, we revisit this subject and focus on the effects caused by the presence of a contact line. In chapter 4 we provide an explanation for the long lifetimes of surface nanobubbles. The hypothesis is based on continuum arguments and invokes an important geometrical property of surface nanobubbles: As these bubbles drain they get flatter due to a pinned contact line, as evidenced in several experiments. This hypothesis is very attractive as it explains, apart from the long lifetime, several other unusual properties of surface nanobubbles. For example, the superstability of surface nanobubbles (i.e., their resilience against brief and strong tensile stresses [2] and strong heating [3]) is completely natural when the contact line of a nanobubble is pinned. The contact angle of surface nanobubbles, that deviates from Young's law, are of course also no surprise when nanobubbles are pinned: Young's law simply does not apply in such a case. The main effect of the pinned contact line is that the relevant length-scale of nanobubble dissolution is not the bubble size, but rather the distance from the drop to the free atmosphere which is several orders of magnitude larger. In closed systems, which have been used in some of the nanobubble experiments, this length can even be considered to be infinite and therefore the nanobubbles would not dissolve at all.

Chapter 5 is the last chapter about surface nanobubbles. Here, we an- 
alyze the growth of nanobubbles in an acoustic field by rectified diffusion by a one-dimensional diffusion model. By seperating the fast timescale associated with the acoustic frequency from the slow timescale at which the bubble grows, we are able to make a detailed phase diagram in which the growth conditions for nanobubbles are elucidated.

The second part of the thesis concerns more general aspects of capillarity, and is primarily focused on the relations between microscopics and macroscopics of fluid-fluid and solid-fluid interfaces. Chapter 6 reconciles microscopic, thermodynamic, and mechanical viewpoints on various aspects of capillarity such as Young's law, the floating pin paradox, and the force on a solid due to the presence of a contact line. We revealed that many of the inclarities (or even paradoxes) are caused by an improper definition of the system on which forces act. For example: When considering the equilibrium contact angle of a partially wetting liquid on a solid (Young's law) one needs to define a proper control volume that contains the liquid around the contact line. This is usually overlooked, and many textbooks contain an (over)simplified depiction of the involved forces. While the textbook examples work for the specific cases that they consider, they cannot be applied in general and should therefore be considered more as mnemonic aid than as a representation of the true physical forces. The chapter also contains the basis for a technique applied in the following chapters (density functional theory in the sharp kink approximation, or DFT) which is a continuum-like description of microscopic interactions.

In chapter 7 we describe a clear manifestation of the finite range of microscopic interactions: Line tension. We studied the effect and origin of line tension using molecular dynamics and DFT. We found that, for a LennardJones liquid, line tension is always negative (hence, promoting expansion of the contact line length) and its magnitude is small: $\sim \mathrm{pN}$. The magnitude of line tension depends on the contact angle of the liquid-solid pair, and using both molecular dynamics and DFT we found that for larger contact angles the two methods agree. For lower contact angles, the details of the microscopic interactions become important, explaining why the molecular dynamics and DFT results differ.

Then, in chapter 8 a thermodynamic approach was used to describe how partially wetting liquids affect the solid with which they are in contact. Specifically, we found that the surface stress $Y$, rather than the surface energy $\gamma$ determines the elastic deformation of an elastic solid due to capillary forces. The result highlights that, in the case of liquid-solid interactions, 
one needs to account for the rheology of the solid. The general inequality $\gamma \neq \mathrm{Y}$ also means that contact angle measurement of the cusp near the threephase line does not provide sufficient information to define the value of the surface stress [4]. The strain-discontinuity across a contact line of a slender body, however, does directly provide the difference $\mathrm{Y}_{S L}-\mathrm{Y}_{S V}$.

The relation between surface stress and surface energy is described in chapter 9. Using DFT, we find that the the key parameter that determines elastocapillary behaviour is the compressibilty (in the surface layer) of the elastic solid: The Poission ratio $v$. We recovered that for liquid-liquid interfaces (incompressible, $v=1 / 2$ ) the surface energy and surface stress are equal: $\gamma=\mathrm{Y}$. This is necessarily true: If this were not the case a residual tangential force would be transmitted to the liquid bulk phases, which cannot be in equilibrium due to the absence of elasticity. In the case of an elastic medium, however, this is possible and generally the case. Therefore, the full relation between $Y$ and $\gamma$ is crucial when one is interested in the elastic deformation of partially wetted deformable surfaces.

In chapter 10, we studied the early dynamics of a spreading droplet both experimentally and using molecular dynamics. Both methods yielded consistent results with a scaling argument that consists of a balance between inertial and capillary forces. More precisely we concluded that, regardless of wettability, the initial spreading radius $r(t)$ of a liquid on a solid that it partially wets always follows the power law $r(t) \sim t^{1 / 2}$, where $t$ is the time after initial contact. These results contradict earlier findings by Bird et al., where a wettability dependent exponent was found [5]. Comparing the data, we found that the experimental resolution of the experiments by Bird et al. was insufficient to recover the early spreading regime, such that it was impossible to properly measure the exponent. The recovered universal behaviour is surprisingly robust: The molecular dynamics simulations reveal that almost immediately after first contact the spreading dynamics follow the aformentioned power law, even though the spreading radius is only a few molecular diameters wide!

\subsection{Outlook}

To conclude this thesis, we now discuss some possible directions for future work on the subjects of this thesis.

We start with surface nanobubbles. The explanation put forward in chapter 4 certainly provides an attractive and elegant solution to many of the 
mysterious properties of surface nanobubbles, as discussed in the summary. The explanation, however, also raises a new question: Why, and to what extend are nanobubbles pinned? Certainly, some contact angle hysteresis is to be expected anyhow, but for surface nanobubbles this effect appears to be stronger than for macroscopic bubbles. The amount of pinning in the case of surface nanobubbles must therefore be quantified by direct measurements of the contact angle hysteresis.

A promising way to determine the mobility of surface nanobubbles was discovered from the experimental data of nanobubble growth by rectified diffusion (chapter 5). We found that, when an acoustic field was applied to nanobubble for some time, some of the nanobubbles got displaced from their initial positoin. Presumably, Bjerkness forces are responsible for these moving nanobubbles, and since there seems to be no clear collective motion towards a fixed (anti-)node position it is most likely that secondary Bjerkness forces are at play. Further experiments to find the threshold acoustic field pressure at which nanobubbles start to move can therefore be used to quantify the pinning strength.

What remains is the question why nanobubbles are pinned, even on smooth surfaces such as HOPG. Is the structure of the substrate altered due to the presence of nanobubbles? Do nanobubbles form at preferential sites? Detailed (dry) scans (for example using scanning tunnel microscopy or scanning electron microscopy) of substrates on which nanobubbles were attached can reveal chemical or topological heterogeneities at former nanobubbles sites which would explain the pinning effect.

A final question regarding nanobubbles that has barely been investigated is that nanobubbles seem to exist exclusively in water. It is unclear whether this is truly the case, or that simply no one has looked in different solvents or was able to form nanobubbles using the same techniques used for water. It is perhaps more difficult to form surface nanobubbles in other solvents, a way to bypass this problem would be to generate surface nanobubbles in the usual way (i.e. ethanol-water exchange), verify that they have formed and then slowly change the surrounding solvent in situ and see how nanobubbles react. It is difficult to change only a single parameter in this way but if nanobubbles persist during the solvent replacement, it is at least clear that they are not unique to water.

We close with a description how the elastocapillary model from chapters 8 and 9 can be used to describe the behaviour of multiple droplets on an elastic substrate. Analogous to the well-known 'Cheerios-effect', where 
floating particles can attract or repel each other by capillary means, a similar effect occurs due to the elastic deformation induced by a neighboring droplet. It can be shown that, using an asymptotic expansion, that for large distance $L \gg R$ between drops of size $R$ the normal component of the surface stress results in a repulsive force. Meanwhile, the tangential component of the surface stress yields a much strong attractive force. From this, it is immediately clear how a correct understanding of surface stress (chapter 8) and how elastocapillary forces are transmitted to the bulk (chapter 9) is crucial to understanding whether two droplets on a soft, elastic medium are attracted or repelled by each other.

\section{References}

[1] K. Ohgaki, N. Q. Khanh, Y. Joden, A. Tsuji, and T. Nakagawa, Physiochemical approach to nanobubble solutions, Chem. Eng. Sci. 65, 1296 (2010).

[2] B. M. Borkent, S. M. Dammer, H. Schönherr, G. J. Vancso, and D. Lohse, Superstability of surface nanobubbles, Phys. Rev. Lett. 98, 204502 (2007).

[3] X. Zhang, H. Lhuissier, O. R. Enriquez, C. Sun, and D. Lohse, Deactivation of Microbubble Nucleation Sites by Alcohol-Water Exchange, Langmuir (in press), (2013).

[4] R. W. Style, R. Boltyanskiy, Y. Che, J. S. Wettlaufer, L. A. Wilen, and E. R. Dufresne, Universal Deformation of Soft Substrates Near a Contact Line and the Direct Measurement of Solid Surface Stresses, Phys. Rev. Lett. 110, 066103 (2013).

[5] J. C. Bird, S. Mandre, and H. A. Stone, Short-Time Dynamics of Partial Wetting, Phys. Rev. Lett. 100, 234501 (2008). 


\section{Samenvatting}

Wanneer een vloeistof, bijvoorbeeld water, in contact wordt gebracht met een vaste stof kunnen allerlei bijzondere verschijnselen plaatsvinden. Enkele van deze verschijnselen worden in dit proefschrift besproken, zoals de vorming van oppervlakte nanobellen en elastocapillaire vervormingen indien er sprake is van een (zachte) elastische vaste stof. De (oppervlakte) nanobellen zijn bijzonder omdat ze zich niet lijken te gedragen als grotere bellen en het is met name de levensduur van deze bellen die vele ordegroottes langer is dan verwacht. Elastocapillariteit is een benoeming voor het veld waar wordt gekeken naar hoe een vaste stof wordt vervormd door de aanwezigheid van een vloeistof dat in contact is met deze vaste stof. In tegenstelling tot nanobellen, die pas in de jaren 1990 zijn ontdekt, werd elastocapillariteit al beschreven in de jaren 1960. Echter, elastocapillariteit wordt nog steeds actief onderzocht en tot op de dag van vandaag bestaan er verschillende interpretaties over hoe exact de wisselwerking tussen vloeistoffen en elastische materialen verloopt.

Hoofdstukken 2-5 van dit proefschrift gaan over de oppervlakte nanobellen. Vanwege de twijfel over de geldigheid van continuum-modellen voor het beschrijven van nanobellen, is veel werk gedaan met Molecular Dynamics (moleculaire dynamica, MD) simulaties. In hoofdstuk 2 gebruiken we MD-simulaties om de vorming en stabiliteit van nanobellen te bestuderen. We beschrijven hoe nanobellen spontaan kunnen ontstaan in een vloeistof die (lokaal) is oververzadigd met opgelost gas. Door Browniaanse bewegingen kan zo'n bel vervolgens neerslaan op het substraat waardoor het een oppervlakte nanobel wordt. Uit de simulaties bleek dat voor niet gepinde nanobellen op atomair gladde oppervlaktes de bellen niet de bijzonder lange levensduur hebben die in experimenten wordt waargenomen: De gesimuleerde nanobellen losten op in minder dan een microseconde. Echter, we vonden wel een mogelijke verklaring voor de lage contacthoek van nanobellen: Wanneer een gas-adsorbaat vormt tussen de bel en het substraat in (ook wel een micropancake genoemd, vanwege de platheid van zo'n adsorbaat) 
wordt de contacthoek sterk beïnvloed: De bel wordt platter. De platheid van nanobellen is een verschijnsel dat ook in experimenten is waargenomen. Om precies te zijn: De vorm (of platheid) van een bel kan precies worden beredeneerd vanuit de betreffende oppervlaktespanningen tussen de vloeistof, damp en substraat (de wet van Young) maar uit experimenten blijkt dat nanobellen altijd platter zijn.

In plaats van oppervlakte nanobellen hebben we ook zogenaamde bulk nanobellen bestudeerd in hoofdstuk 3. Dit zijn bellen die niet zijn gehecht aan een oppervlak maar die in een vrije oplossing zweven. Ook deze bellen hebben we bestudeerd met MD-simulaties, waarbij de bellen werden omringd door andere bulk nanobellen. Door de (nabije) aanwezigheid van deze andere bellen wordt diffusie, waardoor zo'n bel normaal gesproken binnen een microseconde zou oplossen, effectief onderdrukt. Met behulp van standaard diffusiemodellen kunnen we dit verschijnsel kwantitatief verklaren.

Deze bulk nanobellen zijn dus goed te beschrijven met continuum modellen. Aangezien het grootste verschil met oppervlakte nanobellen de aanwezigheid van een substraat is (en dus, de aanwezigheid van een contactlijn) bekijken we oppervlakte nanobellen nogmaals in hoofdstuk 4 waarin we focussen op deze contactlijn. Uit experimenten is gebleken dat de contactlijn mogelijk (deels) gepind is, d.w.z. de contactlijn verplaatst niet als de bel groeit of krimpt. In hoofdstuk 4 verklaren we met dit gegeven de lange levensduur van nanobellen: Doordat nanobellen steeds platter worden als ze oplossen (vanwege de gepinde contactlijn) lossen ze steeds langzamer op omdat de Laplace-druk afneemt. Aan de hand van een diffusiemodel tonen we aan dat nanobellen hierdoor vele uren en zelfs meer dan een dag kunnen bestaan, zoals ook in experimenten is aangetoond. Het model verklaart ook nog enkele andere eigenschappen van nanobellen zoals de platheid en het feit dat ze bestand zijn tegen trekkrachten veroorzaakt door een negatieve drukpuls en is daarom een zeer aantrekkelijke en waarschijnlijke verklaring voor het afwijkende gedrag van nanobellen ten opzichte van reguliere bellen.

Hoofdstuk 5 is het laatste hoofdstuk over nanobellen. Hierin tonen we theoretisch en numeriek aan dat wanneer bellen worden onderworpen aan een akoestisch veld (ultrageluid) er een proces optreedt waardoor deze bellen kunnen gaan groeien: Rectified diffusion. Dit verschijnsel is al uitgebreid beschreven voor sferische bellen, en in dit hoofdstuk passen we dit toe op oppervlakte nanobellen. Aan de hand van deze analyse stellen we een gedetailleerd fase-diagram op waaruit duidelijk wordt hoe verschillende 
parameters (zoals de oppervlakte spanning of de sterkte van het akoestische signaal) bepalen wanneer bellen groeien door rectified diffusion.

Het tweede gedeelte (hoofdstukken 6-10) van deze thesis behandelt andere capillaire verschijnselen. In hoofdstuk 6 worden de microscopische, thermodynamische, en mechanische aspecten van enkele typische capillaire verschijnselen behandeld zoals de wet van Young, de 'floating pin' paradox, en de krachten op een vaste stof door de aanwezigheid van een contactlijn. Bij capillaire verschijnselen wordt vaak gewerkt vanuit een mechanisch perspectief en veel onduidelijkheid wordt veroorzaakt wanneer niet op een juiste manier een systeem wordt gedefinieerd waarop de krachten werken. Een goed voorbeeld is de wet van Young, waarbij vaak een krachtenbalans tussen de vloeistof/vloeistof-, vaste stof/vloeistof-, en vloeistof/gasoppervlaktespanningen wordt gebruikt. In dit geval is het systeem niet duidelijk gedefinieerd, en in sommige gevallen is deze balans dan ook niet geldig. Veel van de misverstanden, die soms zelfs als paradoxen worden beschouwd, zijn daarom te verhelpen of uit te leggen door de krachten op een correcte manier toe te passen.

In hoofdstuk 7 wordt line tension (lijnspanning) beschreven. Dit is een verschijnsel dat optreedt door het eindige interactie-bereik tussen atomen, waardoor de vrije energie in de buurt van een contactlijn afwijkt van de vrije energie geassocieerd met de oppervlakte-energie. We gebruiken zowel MD als Density Functional Theory (DFT) om de line tension te bepalen van een druppel dat rust op een substraat. Het resultaat was dat, voor eenvoudige (Lennard-Jones) vloeistoffen, line tension negatief is en klein; van de orde picoNewton. De sterkte van de line tension hangt af van de contacthoek: Voor hoge contacthoeken (hydrofoob substraat) volg uit beide methodes dat de sterkte kleiner wordt. Voor kleine contacthoeken hangt de sterkte van de line tension sterk af van de interactie-parameters tussen de vloeistof en het substraat.

Hoofdstukken 8 en 9 gaan over elastocapillariteit. In hoofdstuk 8 herleiden we via een thermodynamische route hoe een elastisch materiaal wordt beinvloed door de aanwezigheid van een contactlijn. De hoofdconclusie is dat niet de oppervlakte-energie, maar de oppervlaktespanning bepaalt in hoe een elastich materiaal vervormd door de aanwezigheid van de vloeistof. In problemen waar elasticiteit geen rol speelt (bijvoorbeeld wanneer twee vloeistoffen in contact zijn) zijn oppervlakte-energie en oppervlaktespanning gelijkwaardig, maar het is al langer bekend sinds de jaren 1950 via Shuttleworth's relatie (hoofdstuk 1) dat dat niet geldt in problemen waar 
elasticiteit een rol speelt. Het werk in dit hoofdstuk toont dus aan dat in elastocapillaire problemen het belangrijk is om de twee te onderscheiden. In hoofdstuk 9 gebruiken we vervolgens DFT om een relatie te verkrijgen tussen oppervlakte spanning en oppervlakte-energie. Het blijkt dat de zgn. Poisson-ratio (i.e. de compressibiliteit van de vaste stof) bepaalt hoe de twee zijn gerelateerd. In de limiet waarin de vaste stof incompressibel is (vergelijkbaar met een vloeistof) zijn de oppervlaktespanning en oppervlakteenergie aan elkaar gelijk, maar in alle andere gevallen niet.

Als laatste, in hoofdstuk 10, bekijken we de gebeurtenissen vlak nadat een druppel in contact is gebracht met een vaste stof aan de hand van MDsimulaties en experimenten. Het belangrijkste resultaat is dat vrijwel direct na contact, de druppel spreidt op een universele manier. Dit betekent dat, zolang visceuze effecten niet domineren, de spreidingsradius $r$ als een functie van tijd $t$ aan elkaar gerelateerd zijn via $r(t) \propto t^{1 / 2}$, waarbij het niet uitmaakt welke vloeistof of wat voor substraat er gebruikt wordt. Dit spreekt eerdere resultaten van Bird et. al. tegen, waarbij experimenteel een spreidingswet werd gevonden die afhangt van de contacthoek. Wij tonen aan dat het verschil veroorzaakt wordt door het beperkte tijdsbereik en de beperkte tijdsresolutie in deze experimenten, en dat de spreidingswet wel degelijk onafhankelijk is van contacthoek voor kleine $t$. 


\section{Acknowledgements}

The work described in this thesis was by no means possible without the help, input, encouragement, and support from many people whom I would like to thank. The project started with an e-mail by and subsequent meeting with Detlef who saw in me a good candidate to participate in the ongoing nanobubbles-project. Looking back, I am very happy that I was offered this opportunity by you, Detlef, and that you would find time in your busy schedule to discuss progress and provide feedback on manuscripts and talks. Your PoF group provides a great working environment for a (starting) scientist, and it is this place that has basically taught me everything I need to know on how to do research and how to keep learning. I am also very grateful that you allowed me to explore other projects which were not directly related to nanobubbles, I really enjoyed working on a broader range of topics, mainly with Jacco.

Jacco, initially we worked together on nanobubbles but it did not take long before you could interest me in some side-projects. These 'side-projects' turned out to become a significant part of this thesis and I'm happy it did, I've always considered working with you a great pleasure. It would be impossible to list everything that you've thought me over the past years but I've always been impressed by your uncanny ability to explain complicated subjects to me or others, be it through one of your whiteboard-sessions or by e-mail. Often, a talk or manuscript that I had prepared would be proofread by you and with some small (or sometimes larger) changes you would be able to make it so much clearer. I am also very grateful that you would make time to discuss matters, and I think the sometimes lengthy e-mail discussions (with many "see attached plot"s) can attest to this.

Of course, there are many other collaborators. For the nanobubble project (chapters 2-5) I would like to thank Robin, Erik, Harold, James, Ko, Xue Hua, Michiel, Siddhartha, Bram B, Stefan, Bene, and Andrea for the many nanobubble-meetings which have been very stimulating. I would like to mention Harold once more for all that he did in the past nine years: $\mathrm{He}$ 
has been my mentor during the Applied Physics study, took place in the graduation committee for my Master defence and now also in the committee for the PhD defence. I would also like to mention James for all the effort he has put into the nanobubble-project, his enthusiasm while doing that, and the direct collaboration on chapter 3 of this thesis. Ko, we already briefly met in 2004 for a small project, and during the last few months while you were visiting the group, we did some great work on chapter 5. I really learned a lot and our meetings were always great fun, be it in the group, on the bike, or 'het Tuinhuisje' and whether it was about rectified diffusion or something completely unrelated such as the sultan's dowry probl... I mean the best choice problem. I hope we work a bit more together and solve this elusive rectified diffusion problem.

For the other projects I would like to thank Antonin M, Antonin E, Bruno, Koen, and Luuk. Antonin M and Bruno for the collaboration on chapters 69. Koen and Antonin E for the collaboration on chapter 10 and for being great friends. Luuk for the current work we are doing on elastocapillarity (not in this thesis). I enjoyed the two years that I've done the werkcolleges for the course 'Fysica van Vloeistoffen', largely due to co-FvV-ers Koen, Wilco, Oscar, Roeland, and Jacco. The group would not function without the secretary and the technicians. I would like to thank Joanita for taking care of the surprising amount administrative tasks that come up during four years. Bas, thank you for all the help in getting me started on Kefalotiri and all the other computer trouble that inevitably happen.

The past four years at the group have been great, and I'll be sad to leave because that means leaving behind some great colleagues and friends. I start with the (mostly) left-handed vegetarian office: Oscar, Hanneke, Varghese, and Laura Schmidt. I consider myself extremely lucky to have (had) you as office-mates, I truly consider our office the \#1 office, not because of its location in the corridor but because of the people in it. Antonin, Mark-Jan and Sander W, thank you for putting up with the +1 you get with Tess as an office mate. Hanneke, Antonin, Koen, Oscar, I really enjoyed spending time with you after office-hours having a drink in the city, climbing, having a BBQ in the garden, or scraping paint from various places in our house.

Many thanks to Jan\&Kitty, for the many weekend dinners but also the care after the snowboard-injury and help with the house. Also of course many thanks to my parents and Casper for their continued love and support during the $\mathrm{PhD}$ and in general. Finally, thanks to Tess for being in my life, I cannot imagine a life without you. 


\section{About the author}

Joost Weijs was born on September $16^{\text {th }} 1984$ in Tiel, the Netherlands. He grew up in Zoelen and attended VWO at the Lingecollege in Tiel. He then went on to study applied physics at the university of Twente in 2004. In 2008 he did an internship at the University of Maryland, USA in the Wolfgang Losert group on segregation in granular mixtures, and in 2010 he graduated cum laude on "Nanoscale Drops and Bubbles at a Surface: A Molecular Dynamics approach" at Detlef Lohse's Physics of Fluids group. He continued to work in the group on a PhD-project about nanobubbles and nanoscopic interpretations of various capillary phenomena. 
